Culture della persona:

itinerari

di ricerca

tra semiotica,

filosofia e

scienze umane a cura di

Jenny Ponzo

e

Gabriele Vissio 


\section{Culture della persona: itinerari di ricerca tra semiotica, filosofia e scienze umane}

Jenny Ponzo e Gabriele Vissio (dir.)

DOI: 10.4000/books.aaccademia.10260

Editore: Accademia University Press

Luogo di pubblicazione: Torino

Anno di pubblicazione: 2021

Data di messa in linea: 4 mars 2022

Collana: Collana di studi del Centro Interdipartimentale di Scienze Religiose - Università di Torino

EAN digitale: 9791280136480

\section{(2) OpenEdition}

Books

https://books.openedition.org

\section{Edizione cartacea}

Data di pubblicazione: 1 septembre 2021

EAN (edizione cartacea): 9791280136466

Numero di pagine: 252

\section{Notizia bibliografica digitale}

PONZO, Jenny (dir.) ; VISSIO, Gabriele (dir.). Culture della persona: itinerari di ricerca tra semiotica, filosofia e scienze umane. Nouva edizione [online]. Torino: Accademia University Press, 2021 (creato il 08 mars 2022). Disponibile su Internet: <http://books.openedition.org/aaccademia/10260>. ISBN: 9791280136480. DOI: https://doi.org/10.4000/books.aaccademia.10260.

(C) Accademia University Press, 2021

Creative Commons - Attribuzione - Non commerciale - Non opere derivate 4.0 Internazionale - CC BYNC-ND 4.0 


\section{RIASSUNTI}

"Persona" è un concetto chiave nella cultura occidentale. La ricca sedimentazione di significati che si è verificata nel corso dei secoli guadagna ulteriore spessore nel confronto con altre idee fondamentali come "soggettività", "identità", "relazione". Questa raccolta di saggi esplora il concetto di persona attraverso diverse discipline, come la semiotica, la filosofia, l'antropologia, la pedagogia, la storia delle religioni. A un primo nucleo di contributi in cui prevale la riflessione teorica (con saggi di Ugo Volli, Carlo Capello, Emanuele Fadda, Sara Nosari), segue una seconda parte dedicata all'interpretazione delle idee di grandi pensatori, tra cui Paolo di Tarso, Karol Wojtyła e Gaspard Koenig, con saggi di Stefano Traini, Francesco Galofaro e Gianmarco Giuliana. Infine, la terza parte si concentra sull'analisi di casi pratici, dai videogiochi agli "spettri digitali" lasciati dalle persone dopo la loro morte, dalle app per interagire con Babbo Natale al fenomeno della persona collettiva nello star system, fino alla codifica dello status di personaggi religiosi, in particolare la Vergine, nelle testimonianze delle apparizioni (con saggi di Marco Papasidero, Davide Sisto, Alessandro Agnese, Eleonora Chiais).

\section{JENNY PONZO (DIR.)}

Professoressa Associata di Semiotica presso l'Università di Torino. È responsabile (PI) del progetto NeMoSanctI (ERC StG, g.a. 757314, nemosancti.eu), Direttrice di CIRCe (Centro Interdipartimentale di Ricerca sulla Comunicazione) e Presidente del Corso di Laurea Magistrale in Comunicazione e Culture dei Media.

\section{GABRIELE VISSIO (DIR.)}

È dottore di ricerca in Filosofia. Dopo aver conseguito il dottorato presso l'Université Paris 1 - Panthéon-Sorbonne e presso l'Università di Torino (Consorzio FI.NO) ha svolto attività di ricerca nell'ambito di diversi progetti, tra cui il progetto Ne- MoSanctI. 
Collana di studi del

CENTRO INTERDIPARTIMENTALE DI SCIENZE RELIGIOSE

Università di Torino

collana diretta da

Ilaria Zuanazzi

comitato scientifico

Paolo Cozzo, Valerio Gigliotti, Alessandro Gusman, Clara Leri, Graziano Lingua, Alessandro Mengozzi, Adele Monaci, Jenny Ponzo, Roberto Francesco Scalon

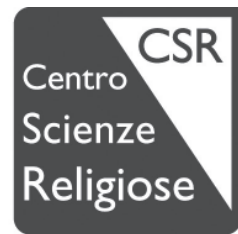

Biblioteca Erik Peterson 


\section{Volumi pubblicati}

1. L'Archivio «Erik Peterson» all'Università di Torino.

Saggi critici e inventario

a cura di Adele Monaci Castagno

Alessandria, Edizioni dell'Orso, 2010

2. Sacre impronte e oggetti «non fatti da mano d'uomo» nelle religioni a cura di Adele Monaci Castagno

Alessandria, Edizioni dell'Orso, 2011

3. Dal Mandylion di Edessa alla Sindone di Torino.

Metamorfosi di una leggenda

Andrea Nicolotti

Alessandria, Edizioni dell'Orso, 2011; edizione rivista 2015

4. Il campo religioso. Con due esercizi

Pierre Bourdieu

a cura di Roberto Alciati e Emiliano R. Urciuoli

Torino, Accademia University Press, 2012

5. Itinerari mediterranei fra Iv e Ix secolo

Città-capitale e Deserto-monastico

Atti del Convegno a cura di Beatrice Astrua

Torino, Accademia University Press, 2013

6. Uomini e orsi: morfologia del selvaggio

a cura di Enrico Comba e Daniele Ormezzano

Torino, Accademia University Press, 2015

7. Marcione e il Vangelo (di Luca). Un confronto con Matthias Klinghardt Pier Angelo Gramaglia

Torino, Accademia University Press, 2017

8. Le porte dell'anno: cerimonie stagionali e mascherate animali

Enrico Comba e Margherita Amateis

Torino, Accademia University Press, 2019

9. Religioni e sviluppo sostenibile

a cura di llaria Zuanazzi e Luca Battaglini

Torino, Accademia University Press, 2021 


\begin{tabular}{|c|c|}
\hline $\begin{array}{l}\text { Culture } \\
\text { della persona: } \\
\text { itinerari } \\
\text { di ricerca } \\
\text { tra semiotica, } \\
\text { filosofia e } \\
\text { scienze umane }\end{array}$ & $\begin{array}{l}\text { a cura di } \\
\text { Jenny Ponzo } \\
\text { Gabriele Vissio }\end{array}$ \\
\hline
\end{tabular}

aA 
Culture

della persona:

itinerari

di ricerca

tra semiotica,

filosofia e

scienze umane
Questo volume fa parte del progetto di ricerca

NeMoSanctI (nemosancti.eu)

che ha ricevuto finanziamenti dal Consiglio europeo della ricerca (CER)

nell'ambito del programma di ricerca e innovazione

Orizzonte 2020 dell'Unione Europea,

in virtù della convenzione di sovvenzione n. 757314

(C) 2021

Accademia University Press

via Carlo Alberto 55

I-10123 Torino

\section{(c) (i) $(9)$ \\ BY NC ND}

prima edizione: settembre 2021

isbn 979-12-80136-46-6

edizioni digitali www.aAccademia.it/culture-persona

https://books.openedition.org/aaccademia/180

book design boffetta.com

Accademia University Press è un marchio registrato di proprietà di LEXIS Compagnia Editoriale in Torino srl 
Parte prima

Approcci teorico-disciplinari

Di che cosa parliamo quando diciamo "persona"?

Fra filosofia e semiotica

L'individuo moderno e il suo Altro... o no?

Un ritorno critico sull'antropologia della persona Carlo Capello 31

'Persona' è l'inverso di individuo?

Qualche riflessione su Peirce e noi

L'«attitudine persona» o della capacità di trasformare:

una prospettiva pedagogica

Parte seconda

Rileggere grandi pensatori

II Gesù divino e inclusivo di Paolo di Tarso.

La ridefinizione di una identità personale

Persona e comunità nel pensiero politico

di Karol Wojtyła. Critica semiotica

di un'analisi fenomenologica

L'Oggettuale e la Persona

nella cultura digitale

Parte terza

Casi di studio

Aspetti e segni della "relazione mariofanica":

il caso delle apparizioni di Banneux (1933) Marco Papasidero 157

Gli spettri digitali della persona:

vivere e mai morire online

B4bb0 N474I3. Lo spettro digitale

di Santa Claus

M¥SS KETA come persona collettiva:

Fenomenologia di una maschera in occhiali, veletta e CAPSLOCK 

Quella della nozione di «persona» è una lunga storia, frutto di una serie di stratificazioni di senso e di slittamenti concettuali, talvolta a prima vista impercettibili, che hanno contribuito a ricollocarne il significato in relazione ad altre importanti nozioni, come quelle di relazione, soggetto o identità. Segno evidente di questa storia di sedimentazioni semantiche è la gran varietà di discorsi e di ambiti in cui la nozione di persona gioca un ruolo importante, all'interno della società contemporanea. Dall'etica al diritto, dalla filosofia alla teologia, dall'antropologia alla letteratura, all'arte, alla politica, non vi è ambito della vita umana in cui sembriamo disposti a rinunciare a questo concetto. Tuttavia, la diffusione del termine non può, in questo caso, essere intesa come segno di un accordo circa il suo significato, anzi: attorno al concetto di "persona" si consumano oggi (per esempio in ambito etico e, specialmente, bioetico) alcuni tra i conflitti intellettuali e culturali più importanti del nostro tempo e, forse, anche alcuni tra i più complessi da decifrare. Infatti, la definizione di che cosa significhi "persona”, la questione di quale sia il valore da attribuire alla persona, nonché quella di chi debba essere considerato persona, sono domande 
che innervano non solo il dibattito intellettuale, ma anche i discorsi che animano la sfera pubblica.

1. La persona: concetto cosmico e posizione di valore

Per meglio chiarire il significato e le ragioni di tale pervasività ci pare di una qualche utilità fare riferimento a una distinzione tratta dal lessico kantiano. Nella Kritik der reinen Vernunft, infatti, al terzo capitolo della Dottrina trascendentale del metodo, dove trova esposizione l'Architettonica della ragion pura, Kant introduce la nozione di «concetto cosmico», che contrappone a quella di «concetto scolastico» (KrV A 840; B868; p. 628) ${ }^{1}$. Se il concetto scolastico è da intendersi come quello di «un sistema della conoscenza, che è cercata soltanto come scienza, facendo astrazione da qualsiasi scopo che non sia quello dell'unità sistematica del sapere, quindi della perfezione logica della conoscenza» (ibid.), il concetto cosmico è invece «il concetto concernente ciò che interessa necessariamente ognuno» (ibid.), il cui fine è «l'intera destinazione dell'uomo, e la filosofia che lo tratta si chiama morale» (ibid.). La nozione di “persona”, potremmo dire allora, appare come un concetto cosmico, nella misura in cui, se da un lato svolge oggi un ruolo chiave nell'elaborazione di un corpus di teorie e di costruzioni concettuali all'interno dell'enciclopedia contemporanea, dall'altro non manca di richiamare questioni di valore, che impegnano sia sul piano etico e morale, sia su quello politico.

Per rendersi conto di quanto tale concetto sia al centro di complesse questioni morali e politiche, è sufficiente osservare come esso sia venuto a trovarsi sistematicamente al centro dei maggiori dibattiti in ambito bioetico. Dalle questioni connesse all'eutanasia a quelle relative all'aborto ${ }^{2}$, per arrivare fino alle tematiche ambientali ${ }^{3}$, la nozione di persona occupa uno spazio di grande rilevanza sia nel dibattito tecnico e specialistico, sia nelle altre forme di discorso che impegnano lo spazio pubblico; e questo avviene perché, po-

1. Le citazioni dal testo kantiano rimandano, nell'ultimo riferimento in parentesi, alla traduzione di Pietro Chiodi indicata in bibliografia.

2. Per un'introduzione alla questione si veda Mori (2008).

3. Per un'introduzione ai principali temi e problemi dell'etica ambientale, con speciale riferimento alle questioni connesse all'antropocentrismo, particolarmente rilevanti nei dibattiti sul significato di "persona”, si veda Donatelli (2012). 
tremmo dire, quello che qualifica un dato individuo o una certa entità come "persona” è sempre un giudizio di valore, che opera al contempo una valutazione e una valorizzazione del proprio oggetto.

\section{Un termine difficile}

In realtà, proprio la rilevanza del termine in ambito pubblico è indice della complessità semantica e dell'ambiguità teorica che lo connotano. Se, com'è noto, è alla Stoà greca che è lecito attribuire la paternità del riconoscimento del significato di persona come "personalità individuale", che si sviluppa nell'ambito di una comune "natura umana" universale (Pohlenz 2005, p. 409) ${ }^{4}$, il termine vede una complessiva ridefinizione del proprio significato, prima nell'ambito del diritto romano, e successivamente in ambito cristiano, dove svolge un ruolo decisivo nella teologia trinitaria (Lingua 2021). In questo ambito, il termine "persona" viene utilizzato, in primo luogo, non tanto per la definizione del soggetto umano, ma come strumento concettuale indispensabile al discorso teologico e, quindi, in riferimento, innanzitutto, alla realtà divina. Dunque, ben prima degli attuali tentativi di estendere la nozione di persona ad alcune realtà extraumane (come gli animali, le intelligenze artificiali o persino la natura ${ }^{5}$ ), il cristianesimo ha inteso il termine "persona" come riferito in primo luogo a Dio e, di conseguenza, a un'entità non-umana.

In età moderna, poi, il termine ha continuato a veder stratificarsi i suoi significati. È con John Locke e la sua discussione del concetto di identità, per esempio, che emerge la questione filosofica dell'identità personale, ma solo con l'età contemporanea la nozione assume un ruolo cardine nella costruzione di alcuni importanti sistemi filosofici. Così, per esempio, Charles Renouvier ritrova nella personalità

4. Cfr. anche Volli infra.

5. In particolare, sono interessanti (o perlomeno significativi da un punto di vista storico e culturale), i sempre più numerosi tentativi di riconoscimento della personalità giuridica della natura (o di alcune sue componenti). Un caso particolarmente significativo è dato, per esempio, dal Te Awa Tapua Act del 2017 in Nuova Zelanda, che dichiara, all'art. 14, il Te Awa Tupua (nome giuridico con cui la norma indica il fiume Whanganui e «ogni sua componente fisica e metafisica») come «legal person», con i relativi «diritti, poteri, doveri e responsabilità di una persona giuridica». Cfr, Te Awa Tapua Act (Whanganui River Claims Settlement), art. 14 (1). 
[personnalité] non solo la categoria fondamentale, ma anche il punto di partenza obbligato per ritrovare il fondamento dell'uomo nella libertà (Renouvier 1903), che egli intende una verità prima; il suo personalismo arriva così ad assumere i tratti di una concezione cosmologica, oltre che antropologica, che colloca la personalità al centro dell'intero impianto della filosofia. Tuttavia, è con Emmanuel Mounier che, com'è noto, il personalismo raggiunge matura formulazione nella prima metà del Novecento. Il Manifeste au service du personnalisme del 1936, insieme agli scritti che appaiono, a partire dal 1932, sulle pagine della rivista Esprit delineano i contorni di una nuova filosofia, che muove dal riconoscimento del valore assoluto della persona, ben distinguendosi, tuttavia, dalle tentazioni individualiste. "Persona", infatti, indicherà per tutto il personalismo, sino alle sue riprese più recenti - come quella operata da Paul Ricœur ${ }^{6}$ - una realtà relazionale, costitutivamente aperta, in senso dialettico o dialogico, al riconoscimento dell'alterità. Anche Jacques Maritain, nel quadro della sua impostazione neotomista, riconosce una distinzione tra individuo e persona, dove il primo termine finisce per indicare la realtà bio-psicologica del soggetto, mentre il secondo esprime la relazione costitutiva di un dato soggetto con $\mathrm{Dio}^{7}$. In questo senso, dire che l'uomo è persona significa, per il pensatore cattolico, affermare in altre parole che questi è "immagine di Dio" e che riproduce, nella propria esistenza storica e concreta, la stessa natura relazionale e "comunicativa" del Dio trinitario.

La storia della nozione è ovviamente molto più complessa di quanto si possa in questa sede riassumere, ma questi sono solo alcuni esempi dei molti sensi e significati che sono andati stratificandosi nel corso della storia del pensiero europeo e occidentale, ai quali andrebbero aggiunti tutti quei modi di intendere la parola "persona" emersi da contesti, culture e tradizioni diverse ${ }^{8}$. È chiaro, dunque, come spesso

6. Oltre a Ricoeur (1997) si veda anche il suo volume dedicato alla figura di Mounier (Ricoeur 2005), in cui Ricoeur si confronta direttamente con la figura del padre del personalismo francese e con la sua eredità intellettuale.

7. Tra i molti testi che sarebbe possibile citare, ci limitiamo a ricordare La persona e il bene comune (Maritain 1995), nel quale il filosofo personalista affronta con particolare pregnanza la distinzione tra "persona" e "individuo", caratterizzando la prima come progetto di sviluppo spirituale aperto alla relazione con l'altro.

8. Su questo tema si interroga, in particolare, l'antropologia della persona. Si veda, in 
accade, che una tale ricchezza di significati comporti anche ambiguità e difficoltà nell'uso di un simile concetto, che non di rado si aggravano in ragione dell'elemento valutativo che la connotazione di "persona" comporta nei confronti della realtà cui viene applicata, anche quando questa si limita alla sola specie umana.

\section{Classificare e agire}

Le scienze umane, infatti, non hanno mancato di sottolineare, specie a partire dalla seconda metà del Novecento, come la classificazione degli esseri umani interna a ogni società comporti, necessariamente, l'attribuzione, nei confronti delle diverse classi di soggetti, di funzioni e compiti all'interno del gruppo sociale più ampio. Dagli studi sulla parentela, che tanta parte hanno avuto nello sviluppo dell'antropologia culturale, agli studi sociologici sulle classi sociali o sulle élites, ciò che emerge chiaramente è che ogni classificazione all'interno dei gruppi umani stabilisce e regola relazioni di potere, gerarchie o rapporti di forza tra gli individui che le compongono.

Questa strutturazione, tuttavia, appare tutt'altro che semplice, poiché il progresso delle scienze umane e sociali ha sempre più chiaramente rilevato come, all'interno delle società umane, convivano spesso numerosi criteri di classificazione, che necessariamente producono aree di sovrapposizione e di intersezione, di inclusione reciproca o di subordinazione. Se ci si concentra, in particolare, sulle società cosiddette occidentali e, nello specifico, sull'età contemporanea, è quasi banale rilevare come uno stesso individuo possa, di volta in volta, essere classificato sulla base della propria età, della propria identità di genere, ma anche in base al proprio apparato riproduttivo, al proprio lavoro o al proprio grado di istruzione, al proprio stato di salute e ad altri numerosissimi criteri di selezione.

Lungi dal possedere un mero valore descrittivo, tali classificazioni sono caratterizzate da importanti effetti performativi, che determinano la possibilità, da parte degli individui, di agire nel mondo in un dato modo, intrattenere certi tipi di relazione anziché altri, accedere o meno a particolari

proposito, Capello (2016), oltre che, dello stesso autore, il saggio contenuto nel presente volume (cfr. infra). 
privilegi, contesti o situazioni. La posizione che ogni individuo occupa all'interno di questa rete di tassonomie non solo risponde alla domanda sull'identità personale ("chi sei?"), ma anche a quella sull'agency ("che cosa fai/puoi fare?"), nella misura in cui a diversi tipi di persona, diversi kinds of person, rispondono specifiche possibilità di movimento sul piano dell'azione.

\section{Culture della persona}

Tentare di comprendere che cosa significhi definirsi ed essere definiti da altri come persona e come un tipo particolare di persona rappresenta, dunque, un passaggio centrale e obbligato per la comprensione dei fenomeni sociali e culturali del nostro tempo. Soprattutto, questo tentativo difficilmente potrà essere considerato prerogativa di un unico discorso disciplinare: la complessità e la polisemia della persona la costituiscono, al contrario, come un oggetto privilegiato di sperimentazione interdisciplinare e di ibridazione dei saperi specialistici.

Non è un caso, dunque, che il presente volume si apra con una serie di contributi di natura teorica e metodologica che gettano sulla nozione di persona luci provenienti da prospettive disciplinari diverse. Il saggio di Ugo Volli, che si colloca esplicitamente sulla frontiera sottile che separa la filosofia dalla semiotica, si confronta con diversi momenti teorici cruciali nella storia del pensiero occidentale, mostrando come il nodo tematico della persona non comporti solo questioni di ordine teoretico, ma anche - e forse in primo luogo - di natura morale e politica. Tale convinzione trova risonanza nel contributo di Carlo Capello che, muovendo dal saggio di Marcel Mauss del 1938, Una categoria dello spirito umano: la nozione di persona, quella di "io", esplora le difficoltà e il potenziale che la categoria di "persona" offre all'indagine antropologica, in un confronto che, nel tentativo di superare l'identificazione della persona con l'individuo, spinge l'antropologia a ibridare il suo patrimonio concettuale con quello del pensiero critico-filosofico. Al distanziamento del concetto di "persona" da quello di "individuo" contribuisce anche il saggio di Emanuele Fadda che muove da un confronto sistematico con l'opera di Charles S. Peirce tornando a sottolineare i punti di aggancio tra la ricerca semiotica e quella filosofica, mediati in particola- 
re dall'originale concezione peirciana della "personalità". Chiude la prima sezione del volume il contributo pedagogico di Sara Nosari che insiste nuovamente sulla specificità della persona anche nell'ottica di uno sguardo educativo, nel cui ambito si conferma l'idea che l'essere persona non costituisca una proprietà, per così dire statica, dell'essere umano, ma un compito da realizzare, oggetto privilegiato dell'interesse educativo.

La seconda sezione del volume, invece, offre al lettore la rilettura di alcuni momenti chiave della riflessione occidentale sulla nozione di persona. Senza alcuna pretesa di completezza, le ricerche qui presentate prolungano il quadro di ibridazione concettuale e metodologica della sezione precedente, esercitandolo in esercizi ermeneutici concreti. Così, il saggio di Stefano Traini ritorna sulla questione di lunga tradizione dell'identità personale di Gesù, muovendo da un'analisi dell'opera di Paolo di Tarso. Ciò che rende originale e particolarmente interessante il contributo è precisamente l'apporto metodologico che mira a mettere al lavoro l'armamentario teorico-concettuale della semiotica, collocandolo in un quadro che tiene conto dei più importanti risultati del dibattito sul Gesù storico. Il saggio di Francesco Galofaro prosegue nello stesso spirito interdisciplinare, favorito dallo stesso oggetto di analisi, costituito dall'opera filosofica di Karol Wojtyła. Il personalismo di Wojtyła, infatti, si radica nella tradizione filosofica di lingua tedesca, con particolare riferimento alle ricerche fenomenologiche di Edith Stein e, soprattutto, di Max Scheler. L'integrazione tra la prospettiva fenomenologica e la ricerca semiotica trova così una felice soluzione, che consente a Galofaro di passare dal piano ermeneutico dell'interpretazione del pensiero di Wojtyła a considerazioni di più ampio respiro circa la centralità della categoria di "sequela" in numerosi aspetti che caratterizzano le società contemporanee. Tra gli elementi distintivi di queste società un ruolo non marginale è occupato dai fenomeni connessi a quella che viene ormai comunemente indicata come la "rivoluzione digitale" e proprio a questi temi si indirizza il contributo di Gianmarco Giuliana. Muovendo da un'analisi dei giochi digitali intesi come produttori di una discorsività digitale e computazionale che riscrive il significato di "persona", Giuliana si confronta in particolare con le ricerche di Gaspard Koenig, in un testo che mette 
nuovamente in contatto i metodi semiotici con gli strumenti concettuali forniti dalla filosofia. Ne emerge un'analisi che trascende i confini del caso di studio proposto e approda a conclusioni di ordine generale, sui limiti di una definizione oppositiva di che cosa debba oggi intendersi con "persona" e proponendo una prospettiva, al contrario, partecipativa della nozione.

La terza e ultima parte del volume, invece, propone una serie di case studies provenienti da diversi ambiti disciplinari e interessati ad approfondire fenomeni esemplificativi della complessità che il concetto di persona riveste nelle società contemporanee. Tale complessità emerge chiaramente nel saggio di Marco Papasidero che, attraverso un'analisi storico-antropologica, studia il caso delle apparizioni mariane della Vergine dei Poveri di Banneux del 1933. Lapparizione della Vergine alla piccola Mariette Beco, infatti, consente di mettere in luce i confini sfumati dell'identità personale, mostrando come, nel muovere la narrazione degli eventi, sia centrale tanto la domanda su "chi" sia la persona che appare, sia quella su "che cosa" sia il soggetto dell'apparizione: oggetto, spettro o, forse, persona? Sul confine sfumato tra lo spettro e la persona si concentra anche il saggio di Davide Sisto che analizza, con prospettiva filosofica, il tema complesso, anche dal punto di vista sociale, della gestione della morte e del ricordo nelle pratiche culturali digitali. La natura archivistica del web consente infatti oggi una diversa costruzione della propria identità e una diversa gestione del ricordo che abbiamo degli altri e che desideriamo lasciare di noi stessi; secondo Sisto, queste inedite possibilità - non prive di tensioni ed elementi problematici - sembrano consentire e, anzi, richiedere una riscrittura della nostra idea di persona, che dovrà ben presto fare i conti con le identità multiple e stratificate dei soggetti. Su di una linea di lavoro non distante si muove anche il saggio di Alessandro Agnese, che ricostruisce la trasformazione di un'identità personale fittizia, quella di Babbo Natale, alla luce delle nuove riscritture che essa subisce grazie alle "app natalizie" che consentono agli utenti un'inedita possibilità di interazione e di relazione con Santa Claus. Eredità delle "letterine" che i bambini di tutto il mondo indirizzano da tempo al vecchietto elargitore di doni, queste app si inseriscono in un più ampio processo culturale che, attraverso 
Maritain J.

Bibliografia

Capello C.

2016 Antropologia della persona. Un'esplorazione, FrancoAngeli, Milano.

Donatelli P.

2012 Manuale di etica ambientale, Le Lettere, Firenze.

Lingua G.

2021 Trinity, Number and Image. The Christian Origins of the Concept of Person, "International Journal for the semiotics of Law», in corso di pubbl.

1995 La persona e il bene comune, Morcelliana, Brescia.

Mori M.

2008 Aborto e morale, Einaudi, Torino.

Pohlenz M.

2005 La Stoa, Bompiani, Milano.

Renouvier Ch.

1903 Le personnalisme, Félix Alcan, Paris.

Ricoeur P.

1997 La persona, Morcelliana, Brescia.

2005 Emmanuel Mounier. Lattualità di un grande testimone, Città Aperta, Troina (EN). 

Parte prima.

Approcci teorico-disciplinari

aA 

Culture della persona: itinerari di ricerca tra semiotica, filosofia e scienze umane
Di che cosa parliamo quando diciamo "persona"? Fra filosofia e semiotica

Il lessema "persona", presente oggi nella maggior parte delle lingue indoeuropee, appartiene a un campo semantico molto complesso e articolato in cui compaiono anche parole come "soggetto", "mente" "anima", "spirito", "l'io", "il sé", "psiche", "individuo", "l'essere umano", "coscienza", "identità"; nel linguaggio filosofico seicentesco "cosa pensante", in quello contemporaneo "Dasein” ecc. Spesso questo campo, che per semplicità denominerò di qui in poi con l'etichetta persona, viene opposto a quello, altrettanto complesso, del corpo. Fra i due però troviamo talvolta qualche elemento in comune, per esempio proprio nel termine "persona" e derivati, che in certi casi può indicare l'aspetto fisico del corpo (per esempio "una persona elegante", "un bel personale", ecc.). Entrambi i campi infatti possono essere pensati come parte di un campo più vasto, quello che caratterizza in vari modi gli esseri umani.

Come ha sottolineato molto chiaramente Umberto Eco (1984, pp. 23-26), non è possibile organizzare queste strutture semantiche in maniera univoca e gerarchica (secondo un albero di Porfirio): per esempio il campo dell'umanità può essere suddiviso, oltre che per il criterio corpo-mente che ho 
appena citato, anche per generi (maschile vs. femminile), per età (bambini vs adulti), per provenienze etniche o nazionali, per stati di salute, ecc.; mentre per esempio la nozione di corpo, inteso come oggetto biologico, va ben al di là dell'umano, essendo comune a tutti gli animali, e si oppone così all'inanimato. Pensando al nostro campo della persona, è abbastanza comune segmentarlo in sottocampi, che spesso si strutturano come assi oppositivi veri e propri: per esempio quello che oppone la coscienza all'inconscio, quello che contrasta la sede delle emozioni (spesso denominata anima) alla cognizione e al ragionamento (detta spesso mente); sono poi facilmente contrapposti in maniera sequenziale o tensiva diverse "altezze" spirituali; per esempio nella terminologia aristotelica (De An. II, 2, 414a sgg.) si distinguono un'anima "vegetativa", una "sensitiva" e una "razionale", a sua volta suddivisa in "intelletto potenziale" e "attivo". Queste segmentazioni sono poi ulteriormente articolate nella storia del pensiero occidentale, per esempio nella Scolastica. Il dibattito medievale su questi temi è estremamente complesso e non è possibile riferirne qui. Basti rimandare al De unitate intellectus contra Averroistas di Alberto Magno (in bibliografia nell'edizione 2007) che ha aperto la discussione e al In Aristotelis librum de anima commentarium di Tommaso d'Acquino (in bibliografia l'edizione del 1959), che ne costituisce la trattazione più autorevole.

Sul piano pratico l'asse semantico caratterizza però fondamentalmente in diversi modi gli esseri umani, contrapponendoli al resto degli oggetti del mondo proprio sulla base della loro possibilità di esperienze personali, per essere insomma "qualcuno' invece di qualcosa» (Spaemann 1996). Cercando di caratterizzare inizialmente il campo semantico della persona, troviamo che ne fanno parte parole e locuzioni che cercano di caratterizzare oggettivamente ("dal di fuori") ciò che ciascuno di noi si sente soggettivamente ("dal di dentro"), cioè il flusso di coscienza, emozioni e pensieri: questa è un' esperienza (o un oggetto astratto o una condizione) che però è certamente assai difficile da oggettivare in una cosa precisa, fornita delle caratteristiche tipiche degli oggetti del mondo, prima fra tutte quella di essere esattamente localizzabile - benché spesso per descriverla usiamo spazializzazioni piuttosto vaghe, parlandone in termini di un'"intimità", "interiorità", "spazio interno" ecc. cui accederemmo per mezzo dell'introspezione, cioè di uno "sguardo interiore". 
Di che cosa parliamo quando diciamo "persona"?

Proprio questa difficoltà di includere l'esperienza personale nel campo degli oggetti normali apre il campo alla ricchezza terminologica accennata. Nell'analisi filosofica e psicologica contemporanea peraltro accade spesso che la realtà stessa di tale condizione sia messa in discussione e l'introspezione dichiarata illusoria (per una discussione molto argomentata di tale posizione è utile Lyons 1986). Però è chiaro che esiste un discorso sociale vasto e frequente e generalmente accettato intorno alla condizione di cui stiamo parlando, con forti conseguenze religiose, terapeutiche, letterarie ma anche politiche (Wiley 1994) ed etiche (Spaemann 1996), da cui deriva una vasta testualità autoriferita, per esempio diari, confessioni, meditazioni ecc. (Freeman 1993) o eteroriferita: discorso libero indiretto, poesia lirica e flusso di coscienza in letteratura, riprese soggettive nel cinema, atteggiamenti proposizionali in filosofia, embrayage ed estesie in semiotica, ecc. Di fronte a un tema così complesso, il compito della semiotica consiste dunque certamente non nel confermarne o smentirne la realtà scientifica o metafisica, ma nel discutere il senso che gli viene attribuito nella ricchissima e molto stratificata produzione discorsiva appena accennata che la riguarda nelle diverse culture e innanzitutto nella nostra, cercando di chiarificarne la struttura semantica.

Torniamo per un attimo alla qualificazione del campo semantico della persona in termini di "interiorità", come qualcosa che ci sentiamo dentro, il che sembrerebbe un'attribuzione banale e innocente, per così dire fattuale. In realtà nelle varie culture esistono spesso delle localizzazioni somatiche di esperienze o funzioni caratteristiche del nostro campo; ma significativamente non sempre tale somatizzazione è unitaria: noi diamo per scontato che la sede del nostro "pensiero" sia "nella testa", mentre i "sentimenti" positivi (come l'"amore" e la "compassione") starebbero nel "cuore", le "passioni" negative (come la "rabbia") nella "pancia" (ma anche "sullo" "stomaco" e "nel" "fegato") e inoltre possiamo avere o meno la "spina dorsale" della dignità e le "palle" della volontà. In altre culture questa topologia è spesso molto diversa, per fare solo un esempio nella maggior parte del mondo greco il pensiero era collocato "nel cuore" e non nel cervello, mentre le passioni si ponevano "nel diaframma". Altre localizzazioni sono ancora più complesse, come i "bernoccoli" sul cranio della frenologia ottocentesca (Taylor 
1989). Per fare solo un esempio fra i moltissimi possibili, in un passaggio del Talmud babilonese (trattato "Shabbat", 33b) leggiamo incidentalmente, come premessa proverbiale a un ragionamento su argomenti medici, che «i reni consigliano, il cuore capisce, e la lingua modella la voce che emerge dalla bocca, tuttavia, la bocca completa» il senso.

Il punto comune di questa collocazione metonimica è comunque il tema dell'interiorità. L'uso, abbastanza recente e linguisticamente anomalo per indicare tale significato, del pronome di prima persona cui viene premesso l'articolo determinativo per sostantivizzarlo ("l'io") mostra la forte tensione semantica derivante dall'ossimoro di un soggetto oggettivato, che si figurativizza in un'interiorità esteriorizzata. Si può citare a questo proposito il titolo (ma solo questo) di un libro di poesie di Peter Handke, risalente al 1969: Die Innenwelt der Außenwelt der Innenwelt (Il mondo interno dell'esterno dell'interno). Naturalmente in questo caso l'asse spaziale "dentro/fuori" è solo una metafora, dato che l'anatomia non rivela spiriti o anime o pensieri nel cervello o nel cuore, ma solo eventualmente strutture neuronali che potrebbero esserne il supporto (e anche su questa relazione fra cervello e mente il dibattito è molto ricco: cito solo qui due classici come Dennett 1991 e Edelman 1989).

$\mathrm{E}$ però tale metafora si sovrappone non casualmente all'asse semantico principale: l'idea che noi (proprio noi, il nostro vero essere) siamo dentro la nostra testa è un esempio della "metafora del contenitore", classificata da Lakoff e Johnson (1980, § 6.2) fra le fondamentali "metafore ontologiche" di "orientamento"( su questo tema, si vedano anche Pinker 2007; Jackendorff 1990, 1991). Essa risale almeno all'immagine platonica della pupilla (kore) al centro dell'occhio come «la parte più divina» dell'anima (Alcibiade maggiore 133c) o al tema agostiniano della «vasta caverna della memoria» "piena di pieghe segrete e ineffabili» «dove riposano i tesori delle innumerevoli immagini di ogni sorta di cose introdotte dalle percezioni; dove pure sono depositati tutti i prodotti del nostro pensiero, e tutto ciò che vi fu messo al riparo e in disparte e che l'oblio non ha ancora inghiottito o sepolto» (Agostino di Ippona, Confessioni, X, 8-10). Essa ha, come accennato, un posto importante nel dibattito contemporaneo sulla coscienza (dal "fantasma nella macchina" di Gilbert Ryle 1965 al "teatro cartesiano" di Dennett 1991). 
Di che cosa parliamo quando diciamo "persona"?

È importante ribadire che la segmentazione del campo semantico di cui parliamo è fortemente arbitraria. I termini che ne fanno parte non hanno riferimenti autonomi, ma ritagliano in vario modo una sostanza del contenuto che è continua. È probabilmente impossibile distinguere correlati oggettivi chiari e reciprocamente esclusivi per termini come "mente", "anima”, "soggetto", “persona”. Naturalmente non vi sono, dal punto di vista semiotico, "concetti puri" (idee platoniche universalmente esistenti) o, almeno nel campo storico/sociale, "generi naturali" nel senso proposto da Putnam (1975) e sistematizzato da Ellis (2001). Se per esempio in qualche modo si può parlare di un'essenza dell'acqua o di altre sostanze chimiche (che emerge dalla loro composizione molecolare, da cui dipendono le loro proprietà chimico-fisiche), e affermare che essa sia indipendente dalla sua comprensione da parte delle diverse società nei diversi tempi, e lo stesso si può fare per i generi biologici, ciò non è certamente vero per oggetti sociali come il denaro (Searle, Ferraris, 2018). E neppure per caratterizzazioni come "persona" e le sue varianti, la cui definizione dipende fortemente da pratiche e teorie sociali espresse da quella che si usa chiamare Naive Psychology, teoria psicologica ingenua o "naturale" di una cultura (Goldman 2015; Burke 1969). Non intendo sostenere naturalmente che questi concetti non abbiano rapporti con la realtà o che siano del tutto infondati o soggettivi, né tanto meno che non esistano gli esseri umani con le loro particolarità; ma solamente che non si può presupporre un'esistenza universale dei termini e dei concetti che la descrivono, al di là della singola cultura di riferimento e della sua Enciclopedia (Eco 1979).

Come Eco stesso sapeva bene, però, anche l'Enciclopedia semantica di una cultura è impossibile da descrivere completamente, a causa del suo carattere labirintico e locale. Questo è certamente vero per il campo semantico della persona, così complesso e autoreferenziale. Un buon modo di capire il modo in cui ogni Enciclopedia tematizza i fenomeni storico-sociali (fra cui "mente", "pensiero", "persona") in una certa società e in un certo tempo è di guardare agli usi della lingua, che ne costituisce il "sistema modellizzante primario" (Lotman, Uspenskij 1973). Per questa ragione è interessante studiare il nostro campo semantico, senza 
pretendere una comprensione immediata della "cosa in sé" dello spirito.

Tornando dunque al nostro campo, Buck (1949) mostra come nel complesso delle lingue indoeuropee si trovi spesso una importante sovrapposizione lessicale fra due dei più importanti poli che organizzano il nostro campo in un asse oppositivo fondamentale per la nostra cultura, cioè quello di "anima" o "spirito" (scheda 16.11 del dizionario di Buck; noi oggi diremmo più facilmente: "psiche") in quanto "sede delle emozioni", in opposizione a "mente" (scheda 17.11), intesa come "sede dell'intelligenza". Lessemi come "spirito", "thumos", "anima" ecc. si avvicinano a seconda del tempo, della lingua, dell'autore o del contesto all'uno o all'altro polo. Per fare un altro esempio, il lessema "soggetto" (sub-jectum, affermatosi come traduzione latina del greco upo-keinomenon, entrambi composti da un verbo causativo di dislocazione come "gettare" e da una specificazione spaziale diretta al basso come "sotto"), per la maggior parte della storia della parola è stato usato per significare un argomento principale "sottoposto" alla discussione, o una "sostanza" affetta da attributi superficiali o perfino un "suddito" - tutti significati derivanti dalla metafora implicita di qualcosa buttato giù/davanti/sotto (Abbagnano 1971; Ayto 1990). Solo con Kant "soggetto" arriva a designare l'"io conoscente". Ancora: "individuo" (che significa a lungo solo attributivamente qualcosa di "indivisibile", essendo la traduzione latina del greco atomon, che da Aristotele viene usato per caratterizzare le specie non scomponibili ulteriormente in contrapposizione ai generi che invece da esse sono composti) diventa solo a partire da Leibniz una cosa particolare e solo a partire dalla fine dell'Ottocento si applica ai singoli esseri umani per sottolinearne unicità e indipendenza (Abbagnano 1971).

È chiaro che il campo semantico della "mente" ha una larghissima e probabilmente universale diffusione nelle lingue umane, spesso determinando anche sviluppi grammaticali importanti come la contrapposizione fra genere maschile e femminile, da un lato, adatti solo agli esseri umani, e dall'altro il genere neutro che si applica alle cose - una contrapposizione che peraltro non ha valore universale, essendo presente per esempio in latino, inglese, tedesco, ma non in italiano o in ebraico che non usano il genere neutro. Un altro dispositivo linguistico simile è il pronome 
Di che cosa parliamo quando diciamo "persona"?

di terza persona impersonale ("esso", "ciò", "it", "es", "ça"), anch'esso contrapposto a quelli maschili e femminili. O ancora si può considerare l'esistenza di "criptotipi" semantici (Whorf 1945), che analogamente limita l'applicabilità di certi verbi "soggettivi" (per esempio "capire" o "pensare") a soggetti grammaticali che rientrino in questo campo. Anche da questi riferimenti grammaticali possiamo supporre un riferimento ontologico forte per i nomi di cui stiamo parlando, possiamo pensare cioè che in tutte le culture vi sia se non una cosa, almeno un'esperienza che sia in qualche modo individuata e caratterizzata dal nostro campo semantico in opposizione alle cose del mondo, e che quindi in qualche modo essa sia davvero universale.

Questa contrapposizione semantica si rispecchia del resto nel classico problema del solipsismo che si ripresenta di frequente nella storia della filosofia: dando per scontato che io abbia percezione immediata ("interna") della mia soggettività, come posso essere sicuro che gli altri esseri umani (ed eventualmente altri enti) ne abbiano una, dato che li percepisco solo in maniera radicalmente diversa da come conosco me stesso ("dall'esterno")? Non ci interessa naturalmente discutere qui di questo tema e della sua storia. Basti ammettere che nella nostra esperienza e nelle descrizioni che ne troviamo, vi sia qualcosa come questa dimensione. Il linguaggio però (e dopo di esso la filosofia, la teologia, la psicologia ecc.) va più in là e caratterizza in maniera molteplice tale realtà, segmentando variamente il campo semantico e polarizzandolo in assi oppositivi, come quello che abbiamo visto fra psiche e mente, o l'altro fra mente e coscienza, intelletto personale e assoluto, o fra soggetto e persona. Queste opposizioni sono fortemente dipendenti dalla cultura, quindi dal linguaggio e dal momento storico.

Sul piano pratico il nostro campo semantico caratterizza dunque in diversi modi gli esseri umani nello sforzo di catturare la specificità dell'essere umano rispetto alle cose, anche usando diverse caratterizzazioni a questo fine. Il problema è capire quali aspetti dell'umanità siano catturati dai diversi termini nei diversi contesti (perché il senso degli stessi termini cambia quando si modifica il campo complessivo e dunque molto spesso, talvolta da autore a autore). Qui è decisiva l'antropologia (e perfino la teologia e la mitologia) presupposta da ogni cultura. È più facile, per esempio, 
che oggi qualcuno consideri di essere un io (e magari fornito di un "conscio" e un "inconscio"), piuttosto che di avere un'anima, come pensavano di averla tutte le generazioni fino ai nostri bisnonni (e qui bisogna badare anche alla differenza fra ciò che si è e ciò che si ha in questo campo); o pensi di essere un banale individuo piuttosto che un filosofico soggetto, salvo naturalmente che ci si senta accusare di essere un "cattivo soggetto", il che c'entra con la persona ma è tutt'altra cosa. La differenza fra "anima" e "spirito" che a lungo è stata importante in teologia e filosofia, sfugge oggi del tutto anche ai filosofi.

Anche in altre culture il campo semantico è suddiviso in maniera complicata. Secondo i dizionari, in ebraico "persona" si traduce adàm cioè "essere umano", in greco antico so$m a$, cioè corpo, in sanscrito jana (cioè "generatore", "essere vivente"). In ebraico, per approfondire il nostro esempio:

Sebbene l'anima sia intesa in contrapposizione e/o complementarietà al corpo, la cultura biblica ignora tale dualismo e con nefesh esprime tutto l'adam, l'essere umano, quel che gli occidentali intendono per "persona" e non solo il suo elemento psicologico. [...] Viene operata una distinzione interna. In senso stretto nefesh è l'anima sensitiva [...] e poi vi è ruach, lo spirito che individualizza e specifica ogni essere umano [...] A livello ancora più elevato vi è neshamah, l'anima spirituale vera e propria, scintilla della luce divina in ogni persona. (Bertagna, Giuliani 2019, ad vocem "Nefesh")

Ancora un esempio: in Omero, secondo Bruno Snell (1946, p. 30, trad. it.), «la parola $\psi v \chi \eta$ non ha ancora il valore successivo di 'anima', ma più quello di 'soffio vitale che abbandona il corpo alla morte' come dimostrato da Il. XXI, 569 ». Vi sono poi i concetti di $\vartheta u \mu o s$ ("organo dell'anima che suscita le emozioni e spinge gli uomini a muoversi") e voos ("sede dell'intelletto, delle immagini e dei pensieri").

E dunque c'è una complicazione in più: i termini del campo non sono solo polisemici e cambiano senso nel tempo, ma ricevono e perdono continuamente nuovi termini "vicini", come ho esemplificato prima con l'arrivo nella cultura occidentale del "soggetto" o dell'"individuo", che modificano indirettamente il senso di tutti gli altri. Per ricostruire il campo, non risulta dunque utile seguire i singoli termini (i significanti) come se ciascuno di esso designasse continuativamente lo stesso significato o lo stesso concetto, 
Di che cosa parliamo quando diciamo "persona"? anche se senza dubbio la scelta di un nome rispetto a un altro o l'invenzione di un termine nuovo fornisce indizi significativi in particolare sugli interessi e sulle domande che un autore o una cultura fanno all'esperienza fondamentale designata dal nostro campo semantico; bisogna piuttosto lavorare a partire dal piano del contenuto, sebbene anche qui non sia possibile una mappa completa del campo semantico, nella diacronia come nella sincronia. In questo labirinto linguistico e concettuale ciò che è importante, come già sapeva Saussure (1967, pp. 146-147), sono le differenze.

\section{Il dibattito filosofico moderno sull'interiorità}

Partiamo dunque da una delle specificazioni del contenuto del campo semantico, la più diffusa in campo filosofico, per cercare di precisare in seguito un concetto di "persona" che corrisponda meglio al nostro uso, seguendo cioè di qui un percorso oppositivo. Il riferimento importante in questo campo semantico che voglio far emergere a questo punto è il soggetto centro di imputazione e di continuità a una coscienza (riflessiva) individuale di pensieri, rappresentazioni, azioni, passioni, sensazioni, ciò che Paul Ricoeur (1990) definiva col pronome $i d e m$, in quanto contrapposto a ipse. Vi è qui una contrapposizione fra aspetto cognitivo (che riassumerò sotto il termine "mente") e aspetto volitivo-passionale (che chiamerò con un certo consapevole anacronismo "psiche"). Vediamo alcuni suggerimenti filosofici in questo senso, per cercare poi di precisare la nozione che ci interessa su questo sfondo.

Per non estendere troppo l'analisi, vale la pena di limitare il discorso alle origini della discussione filosofica moderna, quel secolo e mezzo cruciale per la storia non solo intellettuale dell'Europa che va dalla metà del Seicento alla fine del Settecento. René Descartes, il primo ad aver posto sistematicamente questo problema nella filosofia moderna, anche quando nelle Meditationes de prima philosophia (1641) arriva a discutere dell'interiorità dell'essere umano e della sua capacità cognitiva, usa la parola "ego" nel senso proprio della prima persona dell'enunciatore, senza sostituirla mai con un nome comune o sostantivizzarla (come quando oggi si usa parlare "dell'" io). Come è evidente nel testo, quando scrive «[ego] cogito ergo sum sive existo» (Meditatio II, De natura mentis humanae: quod ipsa sit notior quàm corpus), non intende parlare "dell'io" astratto e sostantivo ma proprio di 
sé, del cavalier Descartes, sia pure con l'intento di prendersi a esempio di una più generale condizione umana. In quest'opera egli usa solo una volta il termine persona, al termine della IV Meditazione, ma prendendola nel senso di "ruolo sociale" - mentre personne appare una sessantina di volte nel Discours de la méthode (1637), e però sempre in un uso non tecnico, cioè intesa come "qualcuno" o nelle frasi negative "nessuno". Nelle Meditationes vi sono anche due occorrenze di subjectum ma solo nel senso di "argomento". Come è noto il termine generico per la condizione in cui Cartesio scopre attraverso il dubbio di ritrovarsi è invece l'espressione per nulla banale res cogitans, "cosa pensante", dove peraltro il pensiero è inteso in maniera estremamente estesa, per cui oggi parleremmo piuttosto di "mente":

Col nome di pensiero io comprendo tutto ciò che è talmente in noi, che ne abbiamo immediatamente conoscenza. Così tutte le operazioni della volontà, dell'intelletto, dell'immaginazione e dei sensi, sono dei pensieri. (Meditazioni, "Risposte alle seconde obiezioni”)

Pensiero sarebbe dunque tutto ciò che è "in noi" (di nuovo la metafora del contenitore) e inoltre esso sarebbe attingibile immediatamente, una forma di autocoscienza che è data per evidente e non ulteriormente discussa. "Io" dunque, proprio quell'io che Descartes è, non sarebbe altro che la sede di "operazioni" mentali che egli stesso conoscerebbe in qualche modo senza filtri o mediazioni ("clare et distincte"). Si identifica in tal modo un dispositivo ossimorico di riflessione che unisce un pronome indicale soggettivante con una qualificazione ontologica estremamente oggettivante ("io sono una cosa pensante"), il cui centro è nella dimensione mentale del pensiero (perché quella psichica delle emozioni e delle volontà ne è solo una parte). Questa ardita operazione concettuale, in cui il soggetto viene iscritto nella categoria delle "cose", è una delle ragioni determinanti del fascino dell'analisi narrativa (questo significa in fondo $\mathrm{Me}$ ditatio) praticata da Cartesio sulla base del lontano esempio agostiniano.

Pur nel mutamento totale dello stile e anche della terminologia concettuale (dall'ego personale di Cartesio alla mens c'è un abisso) il tema viene ripreso da Spinoza nell'Etica, scritta nel 1662 e pubblicata postuma nel 1677: 
Di che cosa parliamo quando diciamo "persona"?
Pars Secunda "De natura et origine mentis": Propositio XI Primum, quod actuale mentis humanae esse constituit, nihil aliud est, quam idea rei alicuius singularis actu existentis. [corsivo mio]

Ritroviamo qui il paradosso cartesiano della trasparenza della mente, spinta al punto in cui essa non esiste più se non come idea di qualcosa. È un tema spinoso perché porta a chiedersi che bisogno ci sia di raddoppiare l'idea di qualcosa presupponendo una mente umana il cui essere consisterebbe solo di questa idea (e - bisogna pensare - delle altre che vi si succedono): un tema che sarà ripreso da Hume, come vedremo, e che continuerà a turbare la filosofia europea fino alla fenomenologia di Brentano, Husserl e Merleau-Ponty (per una discussione rimando a Franzini 2012), per cui non è mai sperimentare la coscienza "in sé", ma essa è sempre "coscienza di...". Vale la pena di notare che in Spinoza vi è inoltre una sottolineatura assai significativa dell'importanza nella costituzione della persona dello sforzo o desiderio (conatus) e soprattutto della sua (auto)consapevolezza (che naturalmente apre altri problemi: la consapevolezza riflessiva di una pulsione astratta alla perpetuazione dell'esistenza è idea di che cosa?).

Pars tertia "De origine et natura affectuum" Propositio IX. Mens [...] conatur in suo esse perseverare indefinita quadam duratione, et huius sui conatus est conscia. Scholium Hic conatus cum ad mentem solam refertur, v o l u n t a s appellatur; sed cum ad mentem et corpus simul refertur, vocatur a p p e t i t u s [...] cupiditas est appetitus cum eiusdem conscientia. [corsivo mio]

Abbiamo dunque, sia pur nell'ambito del monismo spinoziano, l'accentuazione di due fuochi semantici, la mens costituita da idee e il conatus, la volontà di autoperpetuazione, che però è comune anche al corpo, come si vede dalla definizione, e in definitiva compete a ogni creatura vivente. Per questa ragione, che l'avvicina all'anima sensitiva di Aristotele, e per il suo aspetto emozionale di "appetito" esso è più vicino al polo della psiche.

La tematica dell'autocoscienza riflessiva come caratteristica centrale della soggettività viene ripresa come punto di partenza di una importantissima riflessione di Locke ( $A n$ Essay Concerning Human Understanding, Book II, 27,9). In 
essa probabilmente troviamo per la prima volta il lessema "persona" utilizzato come termine tecnico della filosofia relativamente al nostro campo semantico - mentre essa era già apparsa in tutt'altro senso in filosofi cristiani della tarda antichità e del medioevo, specificamente come traduzione del termine ypostasis riferito ai componenti della Trinità, dunque col significato di "sostanza" (Agostino, De Trin. VII, $6)$, specificata poi da Boezio come «sostanza individuale di natura razionale» (De duabus naturis, 3) e da Tommaso come «la relazione nella forma della sostanza, che è l'ipostasi sussistente nella natura divina» (S. Th. q. 29, a. 4). Nella riflessione di Locke, invece, che risale al 1690, troviamo un tentativo di descrivere le radici e il funzionamento dell'identità personale vera e propria:

To find what personal identity consists in, we must consider what 'person' stands for. I think it is a thinking intelligent being, that has reason and reflection, and can consider itself as itself, the same thinking thing at different times and places. What enables it to think of itself is its consciousness, which is inseparable from thinking and (it seems to me) essential to it. It is impossible for anyone to perceive, without perceiving that he perceives. When we see, hear, smell, taste, feel, meditate, or will anything, we know that we do so. It is always like that with our present sensations and perceptions. And it is through this that everyone is to himself that which he calls 'self', not raising the question of whether the same self is continued in the same substance. Consciousness always accompanies thinking, and makes everyone to be what he calls 'self' and thereby distinguishes himself from all other thinking things; in this alone consists personal identity, i.e. the sameness of a rational being; and as far as this consciousness can be extended backwards to any past action or thought, so far reaches the identity of that person; it is the same self now that it was then; and this present self that now reflects on it is the one by which that action was performed. (Locke 1690, Chapter XXVII, "Of Identity and Diversity") [corsivi miei]

Abbiamo qui una segmentazione del nostro campo semantico: persona, identità personale, pensiero, ragione, riflessione, intelligenza, "sé", che stanno in rapporti funzionali fra loro. Il punto centrale in quest'analisi è la coscienza riflessiva, il fatto che sia "impossibile percepire senza percepire di percepire", cioè avere coscienza di se stessi come per- 
Di che cosa parliamo quando diciamo "persona"? cipienti e come gli stessi che hanno avuto altre percezioni nel passato. Ciò che garantisce per Locke l'identità della persona è dunque la sua coscienza riflessiva, che senza far questione di sostanza, garantisce due aspetti fondamentali, la distinzione dagli altri (a chi appartiene questa sensazione di bianco e di dolce?) e la sua continuità nel tempo. È questo livello secondo e riflessivo che fonda dunque la possibilità e la persistenza di un'identità personale. Non ci sono solo sensazioni pure nella sfera psichica, ma sempre percezioni appropriate riflessivamente, percezioni che appartengono a me che so di percepirle. Contro questa idea della continuità fondata sulla coscienza riflessiva polemizzerà però con grande energia Hume nel 1739:

There are some philosophers, who imagine we are every moment intimately conscious of what we call our Self; that we feel its existence and its continuance in existence; and are certain, beyond the evidence of a demonstration, both of its perfect identity and simplicity. [...] Unluckily all these positive assertions are contrary to that very experience, which is pleaded for them, nor have we any idea of self, after the manner it is here explain'd. For from what impression cou'd this idea be deriv'd? This question 'tis impossible to answer without a manifest contradiction and absurdity; and yet 'tis a question, which must necessarily be answer'd, if we wou'd have the idea of self pass for clear and intelligible. It must be some one impression, that gives rise to every real idea. But self or person is not any one impression, but that to which our several impressions and ideas are suppos'd to have a reference. If any impression gives rise to the idea of self, that impression must continue invariably the same, thro' the whole course of our lives; since self is suppos'd to exist after that manner. But there is no impression constant and invariable. (Hume 1739: A Treatise of Human Nature, Part IV, Sect. VI)

Lidentità personale dovrebbe insomma essere fondata sulla percezione di una continuità, che però non sarebbe percepibile proprio per la sua durata (mentre le percezioni sarebbero istantanee: è il vecchio argomento sul tempo che non si può conoscere direttamente già usato nel celebre brano del libro XI delle Confessioni di Agostino di Ippona). Ma su questa argomentazione per la distruzione dell'autocoscienza Hume non ottiene un consenso definitivo. Kant 
ripartirà esattamente dall'argomentazione di Locke, dandola per indiscutibile:

Il fatto che l'uomo possa rappresentarsi il proprio io lo eleva infinitamente al di sopra di tutti gli esseri viventi della terra. Per questo egli è una persona e in forza dell'unità di coscienza persistente attraverso tutte le alterazioni che possono toccarlo, è una sola e medesima persona. (Anthropologie in pragmatischer Hinsicht [Antropologia pragmatica] [1798] §1) [corsivi miei]

Dopo Kant la terminologia dell'“io", della "coscienza" e dell'"autocoscienza" continua a dominare il linguaggio filosofico nell'idealismo di Fichte, Schelling, Hegel, ma è chiaro che non si tratta più della costituzione del singolo essere umano, bensì di realtà globali o istanze metafisiche che non ci interessano qui. Il tema dell'identità personale e dell'unità della coscienza empirica è lasciato cadere. Tant'è vero che quando, meno di un secolo dopo, Nietszche, nel celebre aforisma 119 di Aurora (Morgenröthe. Gedanken über die moralischen Vorurtheile 1881), nega l'esistenza unitaria della sfera psichica del soggetto, ciò verrà largamente ripreso (per esempio da Freud, a partire da Freud 1923), ma non verrà discusso come un argomento filosofico in continuità con il dibattito concluso un secolo prima, bensì come un'osservazione psicologica:

Per quanto uno faccia progredire la sua conoscenza di sé, nessuna cosa potrà mai essere più incompleta del quadro di tutti quanti gli istinti che costituiscono la sua natura. Difficilmente potrà dare un nome se non ai più grossolani di essi: il loro numero e la loro forza, il loro flusso e riflusso, il gioco alterno dell'uno con l'altro e soprattutto le leggi del loro nutrimento gli resteranno del tutto sconosciuti. Questo nutrimento diventa dunque un'opera del caso; i nostri intimi eventi di ogni giorno gettano, ora a questo, ora a quell'istinto, una preda che viene subito avidamente afferrata, ma l'intero andirivieni di queste vicende sta fuori di ogni nesso razionale con le vicende nutritive di tutti e quanti gli istinti; di modo che subentrerà sempre un duplice fenomeno, l'essere affamati e il languire degli uni, il rimpinzarsi, invece, degli altri. Ogni momento della nostra vita fa crescere alcuni tentacoli del nostro essere ed altri invece li atrofizza, secondo appunto il nutrimento che quel determinato momento porta o no in se stesso. Le nostre esperienze, come si 
Di che cosa parliamo quando diciamo

"persona"? è detto, sono tutte, in questo senso, mezzi d'alimentazione, ma sparsi con mano cieca, senza sapere chi ha fame e chi è già sazio. E in conseguenza di questo casuale nutrimento delle parti, anche il polipo interamente sviluppatosi sarà qualcosa di casuale, come lo è il suo divenire. (Nietszche 1881, § 119)

La fenomenologia ripartirà invece da argomenti analoghi a quelli di Hume. Per quanto riguarda l'asse semantico "dell'intimità" che contrappone - per usare ancora questa denominazione convenzionale - "mente", intesa come organo della conoscenza, e "psiche", pensata come luogo delle passioni e delle scelte, Nietzsche rovescia completamente l'impostazione della filosofia seicentesca, privilegiando la seconda: quel che conta sono gli impulsi all'azione e essi sono dislocati in "istinti" molteplici. Ciò che si propone qui però è di nuovo la questione di un'identità personale, al di là delle singole esperienze. Sul piano cognitivo la domanda era che cosa facesse sì che una certa sensazione fosse "mia", con tutto il carico deittico di questa affermazione, cioè che io, proprio io personalmente, fossi colui che percepisce il color verde (per esempio di un albero) e non ci fosse semplicemente un" "idea" impersonale o una sensazione astratta del verde. E che cosa facesse sì che questo io fosse lo stesso che "prima" aveva visto l'azzurro (del cielo)? Non è una domanda astratta o ininfluente, essa è presupposta da ogni conoscenza: anche il più semplice atto dell'aritmetica, quello di contare, richiede questa continuità: la percezione astratta di una mela non si somma con un'altra percezione analoga, se non si riesce a stabilire che si tratta di due percezioni diverse, che riguardano due oggetti diversi e compresenti. Tutto ciò richiede il riferimento a una continuità di tipo lockiano.

Passando all'altro polo, quello passionale e della volontà, citato da Cartesio fra i pensieri, sottolineato da Spinoza e discusso nell'aforisma di Nietzsche, bisogna chiedersi: che cosa fa in modo che le singole esperienze emotive di cui di volta in volta faccio esperienza siano mie? Perché l'appetito e il sonno che sento allo stesso tempo riguardano la stessa persona? Che rapporto c'è fra loro? Che cosa unisce il dolore di una frattura di dieci anni fa, il piacere di una passeggiata l'altro ieri, il freddo di oggi, la stanchezza di domani? Che cosa permane nel flusso dei desideri e delle passioni? 
Vi è poi la storia delle azioni. Perché è "mia" quella laurea che conquistai quasi cinquant'anni fa, quand'ero del tutto diverso? E la responsabilità: perché dovrei essere punito o premiato per un'azione compiuta magari vent'anni fa, o anche ieri, ma in stato di ebbrezza? E se nel frattempo mi sono "pentito"? Il problema delle passioni e delle azioni è analogo a quello cognitivo.

Hanno ragione Nietzsche (e dopo di lui Freud) oppure Locke e Kant? È indubitabile che esista una pluralità di pulsioni che combattono fra loro in ogni persona. Del resto Goethe scriveva in un verso famoso del Faust: «Zwei Seelen wohnen, ach! in meiner Brust.» ("Due anime abitano ahimè nel mio cuore", Faust 1, Vor dem Tor. Verso 1112). E l'ebraismo parla di due istinti, del male e del bene (yetzer rah e yetzer tov) che coesistono in ognuno di noi. Ma d'altro canto esiste un senso molto chiaro per cui non solo la frattura di cui porto la cicatrice nella coscia sinistra è mia (è un vecchio motivo letterario: Ulisse fu riconosciuto dalla sua vecchia balia Euriclea proprio per una cicatrice sulla gamba), ma anche l'amore che ho vissuto da ragazzo, o quel libro che ho letto con passione senza riuscire a fermarmi per tutta la notte sia altrettanto mio. Lo stesso Ulisse è riconosciuto da Penelope non per una traccia fisica ma per un ricordo, su com'era fatto il letto del loro matrimonio. Del resto oggi più che mai la memoria delle password ci identifica. Questa continuità temporale è una delle condizioni che fa di noi una certa persona, fornita di una precisa "identità" e che nella storia si è formata una certa "personalità". Ma, come abbiamo visto brevemente, questa identità non è affatto scontata.

\section{La persona come presenza sociale}

Ma per parlare di "persona" forse non occorre trovare la conclusione, a oggi inesistente, del dibattito precedente sull'asse mente-psiche, perché "persona", almeno nel suo senso più caratteristico oggi, non va identificata con l" "io" che conosce e vuole, con l'“identità intima" accessibile solo da se stessa in maniera immediata e riflessiva di cui abbiamo parlato finora. Nell'uso prevalente nella cultura contemporanea la parola "persona" ne è distinta dal fatto che queste sono entità o stati privati, inattingibili agli altri in linea di principio e insieme immediati, mentre essa oggi quasi sem- 
Di che cosa parliamo quando diciamo "persona"? pre è usata per parlare degli aspetti pubblici dell'identità di un individuo, quelli che si vedono da fuori, che cambiano nel tempo e accumulano relazioni sociali. Nel nostro campo si delinea dunque un altro asse semantico, quello che contrappone "intimità" a "persona". Per verificarlo, partiamo come è d'uso in semiotica da alcune definizioni dizionariali (indico in neretto le parti di definizione che riguardano più da vicino il nostro asse):

DIZIONARIO GARZANTI (https://www.garzantilinguistica.it/ricerca/?q=persona)

1. ogni essere umano in quanto tale, senza distinzione di sesso, età e condizione; 2. l'individuo considerato per le funzioni che esercita, per la posizione che ricopre nella società; 3. il corpo, la figura, le fattezze fisiche proprie di un individuo; 4. nella teologia cristiana, ognuno dei tre componenti della Santissima Trinità, considerato nella sua realtà individuale; 5. (gramm.) categoria che indica il soggetto grammaticale di un'azione.

SABATINI COLLETTI (https://dizionari.corriere.it/dizionario_italiano/P/persona.shtml)

1 Essere umano considerato in sé o nelle sue funzioni sociali, prescindendo dalle differenze di etnia, sesso, età, cultura ecc.; 2 Individuo come presenza e aspetto fisico, come corpo e figura; 3 dir. Soggetto di diritto, con diritti e obblighi; può essere un individuo (p. fisica) oppure un ente o una società (p. giuridica); 4 gramm. Categoria verbale e pronominale che indica i protagonisti dell'atto comunicativo sia diretti (come io e tu) sia indiretti (tipicamente, le terze persone); 5 filos. Individuo quale essere pensante che, attraverso la sua volontà, i suoi sentimenti e l'autocoscienza, rappresenta l'essenza dell'uomo; 6 Nella teologia cristiana, ognuno degli enti divini formanti la Trinità.

HOEPLI (https://dizionari.repubblica.it/Italiano/P/persona.html)

1 Ogni individuo della specie umana; 2 L'uomo inteso nella sua dignità personale, come portatore di valori umani universali; 3 L'individuo, in quanto membro della società, inteso nelle sue qualità particolari e nelle funzioni che svolge in seno a essa; 4 Il corpo, in relazione al suo aspetto esteriore; 5 Personaggio di un'opera teatrale; 6 DIR L'essere umano inteso come soggetto di diritto; 7 LING Categoria grammaticale; 8 TEOL Ciascuno dei tre componenti della Santissima Trinità. 
ABBAGNANO (1971)

Nel senso più comune del termine: l'uomo nelle sue relazioni col mondo e con se stesso. Nel senso più generale (in quanto la parola è stata applicata sia a Dio che all'uomo): un soggetto di relazioni.

Come suggerisce Abbagnano (1971), anche nel seguito della sua voce "persona", il punto di questo concetto è la relazione fra l'essere umano e il suo contesto, che implica sempre un legame con gli altri, dunque un punto di vista esterno, una dimensione sociale invece che intima. Questa dimensione è messa in evidenza in tutte le definizioni considerate: ovunque c'è un accenno al ruolo sociale, al corpo, alla funzione, cioè a un essere umano com'è presente in società. Mentre il punto di vista implicito (un esempio astratto del "sistema guardante/guardato" di Eugeni 1999) della discussione del paragrafo precedente era interno e riflessivo, qui esso si sposta fuori e inquadra quel che gli altri vedono del soggetto.

Lo si vede anche nell'etimologia della parola che deriva in quasi tutte le lingue indoeuropee dal latino persona, e di lì, forse attraverso una mediazione etrusca, al greco

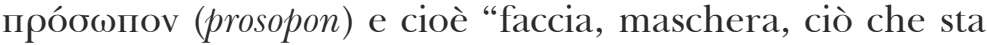
davanti alla vista" (non è chiaro se nel senso di ciò che è più visibile dell'altro o ciò che copre i suoi occhi). Una etimologia alternativa della parola latina, che rimanda sempre alla maschera, parte da sonare: persona $\leftarrow$ per sonare. Essa deriverebbe dal fatto che le maschere antiche servivano anche da megafoni. In ogni caso col nome persona il latino indicava un attore di teatro e per estensione un essere umano visto dall'esterno, in relazione alla società. Gli autori più rilevanti che ne promuovono l'uso sono Cicerone e Seneca quasi sempre in relazione al teatro. In italiano il termine è comune fin da Dante e Boccaccio, spesso col significato generico di "qualcuno"; in inglese si diffonde nel xiII secolo dal francese (che lo riprende dal latino).

Il tema della persona come dimensione sociale del soggetto è al centro di un celebre saggio di Marcel Mauss (1938) che discute il modo in cui la categoria di persona è sorta e si è sviluppata in diverse culture. All'inizio dell'articolo troviamo uno slittamento significativo verso un problematico essenzialismo. A partire dall'esistenza presso gli indiani Zuni e altri "primitivi" di una serie di nomi con cui si configura un soggetto Mauss deduce che cosa sia in quelle società 
Di che cosa parliamo quando diciamo "persona"? una persona: essa consiste innanzitutto nel determinarne il clan, la famiglia e l'età (difatti il nome cambia con l'età o a seconda della sua condizione matrimoniale). Si nomina una "persona", cioè, non tanto per individuarla quanto per rendere noto il suo posto nel gruppo. Abbiamo dunque un uso dei nomi non secondo la designazione rigida (Kripke 1980) cui siamo abituati noi coi nostri nomi propri, ma relativamente a una certa posizione in un gruppo. Vi è persona a pieno titolo solo quando essa assume rappresentanza del gruppo presso la società. La persona vera sarebbe dunque il gruppo e non l'individuo che la rappresenta. Come si vede si tratta di una caratterizzazione indiretta, perché queste culture certamente non formulano un concetto di persona, e non hanno probabilmente un significante univoco che corrisponda in maniera esclusiva a questo termine (almeno il saggio non ne parla).

Peraltro anche noi usiamo diversi elementi linguistici per definire le persone in rapporto alla loro posizione sociale e all'appartenenza a certi gruppi: per esempio con titoli come "lei/tu, signora/signorina, sig./dott./ing./prof." ecc., con la delega alla "legale rappresentanza" di istituzioni collettive, assegnata al loro presidente, e in fondo anche con la differenza fra nome e cognome. I ruoli sociali così definiti sono per noi in qualche modo aggiunti a un'identità personale che riteniamo stabile e principale, ma costituiscono certamente parte della personalità di ciascuno. Mauss in sostanza sottolinea come diverse società antiche trattano i loro membri non tanto in quanto individui unici ma come membri di certe famiglie e clan, dotati di certi ruoli sociali ecc. - e lo fa a partire dal dato linguistico dei nomi. Anche accettando questa osservazione, che andrebbe verificata forse meglio di quanto Mauss abbia potuto fare attingendo velocemente a ricerche etnografiche già fatte per altri fini, la tesi di Mauss in questa prima parte del suo articolo resta discutibile. Se noi consideriamo infatti che questa caratterizzazione esterna delle tribù citate definisca ciò che noi chiamiamo "persona", possiamo dire che sono state esaminate società che privilegiano la dimensione della persona (intesa come ruolo) su quella del semplice individuo. Ma si potrebbe ragionare a rovescio, dicendo che in queste società la dimensione personale è subordinata a quella sociale. Limportante è non pensare che vi sia un'essenza-persona che si manifesta più o 
meno nelle diverse culture: siamo di fronte a un campo semantico più o meno universale, ma variamente strutturato nelle varie culture, i cui termini possono anche non trovare traduzioni esatte in società che privilegiano altri criteri.

Ci interessa di più il fatto, sottolineato da Mauss (1938) nel seguito del suo articolo, che in epoca latina la nozione di persona acquisisce una base giuridica, anche perché i romani usano proprio la parola "persona". Sono infatti essi a mutare il senso di un termine che in origine significava "maschera", in quello di "personalità giuridica" (o "civile"), esteso alle famiglie libere (e in particolare al loro "pater") ma non agli schiavi. Alla persona civile veniva riconosciuta la titolarità dei propri antenati, di un nome, di certi beni e infine, del proprio corpo. In seguito l'influenza stoica apportò una probabile ulteriore innovazione all'idea di persona suggerendo l'esistenza di un'essenza privata (il soggetto, l'io) al di sotto dell'aspetto pubblico - cioè della maschera. Non è importante qui seguire nei dettagli Mauss, anche se vale la pena di sottolineare l'importanza della dimensione giuridica della "persona" che egli individua a partire dal diritto romano: le categorie giuridiche formali infatti rendono precise intuizioni che sono già attive in una semiosfera, sono riconoscimenti semantici ordinati e duraturi di una certa Enciclopedia. "Persona", in questa accezione, è chi dispone di diritti ed è perciò suscettibile di stare in giudizio: non tutti gli esseri umani lo sono, almeno non nel diritto romano arcaico, non i minori, spesso non le donne e gli schiavi, magari i non cittadini. Così avveniva anche ad Atene. In cambio possono essere teorizzate entità collettive cui è attribuita questa capacità (società, chiese, associazioni, persino lo Stato) e così è avvenuto nella storia del diritto europeo. La logica di queste scelte è che alla dimensione personale è presupposta una dimensione strategica, una volontà, una razionalità.

Quel che più interessa dal punto di vista semiotico è la derivazione dalla maschera teatrale. La maschera è un dispositivo di manipolazione della presenza che, ben prima del teatro, compare in moltissime culture anche molto antiche o "primitive" e serve in genere per occultare $\mathrm{o}$, ancora più generalmente, mettere fra parentesi l'individualità di chi la porta ed eventualmente sostituirla con un'altra, più o meno marcata socialmente. Vi sono maschere familiari, maschere 
Di che cosa parliamo quando diciamo "persona"? di gruppo che funzionano come bandiere e anche maschere religiose che intendono affermare la presenza (magari la possessione) di una divinità o di un altro essere umano o sovrannaturale, maschere che affermano un ruolo sociale, maschere che semplicemente rendono anonimi (la maschera neutra, ma anche il fazzoletto sul volto ecc.), maschere che difendono la faccia da intemperie o aggressioni fisiche di vario tipo. Vi sono infine le maschere teatrali, anch'esse largamente diffuse in Grecia, in India (sotto forma di trucco) in Giappone, nella Commedia dell'arte ecc. Qui l'operazione di sostituzione in genere consiste nella costruzione dell'aspetto socialmente codificato di un personaggio (una parola che ovviamente deriva da "persona"). Nella commedia dell'arte, le maschere si sono estese al corpo e al linguaggio, producendo costumi tradizionali, fisionomie, accenti; ormai solo talvolta coprono il volto. Al di là dei dettagli tecnici, quel che ci interessa è il legame fra un certo aspetto fisico e un certo ruolo sociale, che in realtà troviamo anche nella vita quotidiana, per esempio nell'abbigliamento. Nel caso più significativo, la maschera, usando certi dettagli figurativi (per esempio nel Kathakali il colore, in altri casi la forma del naso, la barba) identifica un personaggio noto (che ha una certa personalità o un carattere; si pensi al fatto che in inglese personaggio si dice character).

Tutto ciò suggerisce al semiologo di pensare la nozione di persona in termini analoghi ai personaggi narrativi. Secondo la terminologia della semiotica greimasiana (si veda per esempio Betrand 2000, § 5.2.2.3 e glossario), si parlerebbe piuttosto di "attori", ma per evitare confusioni con il contesto teatrale appena sfiorato continuerò qui a designare coloro di cui le narrazioni raccontano azioni e passioni come "personaggi". L'attore infatti nel teatro, nel cinema ecc. è il supporto corporeo di molti possibili personaggi, come l'oggetto maschera di molte possibili caratterizzazioni. Come è noto questo "personaggio" narratologico secondo l'ipotesi greimasiana è costituito dalla sintesi di un ruolo attanziale e di un ruolo tematico. Il primo è un'unità della sintassi narrativa, che descrive il tipo di funzione svolta in un certo momento dal personaggio, il secondo appartiene alla semantica e ne definisce le caratteristiche concrete, come il sesso, l'età, la professione, fino a dar luogo a figure ricche di dettagli anche sull'aspetto fisico. Ma tali ruoli variano conti- 
nuamente nel corso della narrazione. Per fare un esempio molto ovvio, nei ruoli tematici dei Promessi sposi Renzo è all'inizio fidanzato e poi sposo, prima contadino e poi fuggitivo, rivoltoso, presunto untore ecc.; sul piano attanziale è soggetto del suo programma di matrimonio, oggetto di valore di Lucia ma opponente nel programma narrativo di Don Abbondio quando questi cerca di evitare il matrimonio, e così via. Cappuccetto Rosso è aiutante della madre, soggetto di una passeggiata nel bosco, oggetto dell'appetito del lupo. Se passiamo ai ruoli tematici, è figlia obbediente, bambina distratta, nipote affettuosa, vittima ecc.

Dunque bisogna pensare il personaggio come $i l$ punto di attacco di una serie coerente di ruoli tematici e attanziali e inoltre come il titolare di una serie di programmi narrativi. Se le narrazioni sono sempre agonistiche e i loro sviluppi (gli schemi narrativi canonici che vengono sviluppati davvero nei loro mondi possibili) derivano dagli incontri e dagli scontri di programmi narrativi diversi (quello del soggetto e dell'eventuale antisoggetto, ma anche quelli del destinante, degli aiutanti ecc., ognuno col loro oggetto di valore e il loro contratto implicito) bisogna sottolineare un punto che spesso la semiotica trascura, e cioè che solo i personaggi (nel linguaggio di Greimas, gli "attori”) possono fare da supporto a questi programmi narrativi. Una storia non è mai lo sviluppo meccanico di un corso di eventi, ma sempre il risultato di progetti messi in gioco dai personaggi; o almeno noi non possiamo interpretare che così una storia, se la comprendiamo come sensata. Il personaggio (l'“attore di Greimas, non l'“attante" che è semplicemente un ruolo sintattico passeggero) è quell'entità più o meno umanizzata, che esprime un programma narrativo e lo tiene fermo o progressivamente lo modifica sulla base dell'esperienza e compie azioni, subisce passioni, è coinvolto in situazioni, su questa base. Renzo prova a sposarsi, ma poi decide di fuggire, poi ancora fa ritorno - secondo la necessità degli eventi. Questa collezione di programmi narrativi si ritrova in ogni storia e in ogni personaggio.

Sul funzionamento degli "attori" o personaggi la semiotica ha lavorato poco e teorizzato ancor meno, anche perché essi si situano a livello della "manifestazione", ma in realtà troviamo un disinteresse analogo all'origine della narratologia, nella Poetica di Aristotele (1450a: 15): 
Di che cosa parliamo quando diciamo

"persona"?
La tragedia infatti è imitazione non di uomini ma di azioni e di vite, e dunque non è che i personaggi agiscono per rappresentare i caratteri, ma a causa delle azioni includono anche i caratteri, cosicché le azioni e il racconto costituiscono il fine nella tragedia.

Ma il problema è centrale: senza l'aggancio dell'attore (o quasi sempre di più attori che interagiscono fra loro), le azioni non hanno senso. Non c'è bisogno di leggere psicologicamente le narrazioni per vederle come organizzate da progetti che si scontrano, collaborano, si ignorano, cambiano, sono subordinati l'uno all'altro. Questi progetti, nella maggior parte dei casi prima della grande narrativa moderna, sono visti solo dall'esterno, epicamente. Il personaggio non è di solito un soggetto psichico, ma di azioni. Azioni sensate però, che appaiono espressione di progetti.

Dato che noi interpretiamo naturalmente in maniera narrativa la nostra esperienza del mondo, questa attribuzione di progetti è il modo in cui noi esploriamo e spieghiamo anche le vicende che ci accadono. Non importa qui stabilire se aristotelicamente i personaggi siano "imitazioni" delle persone che portano progetti, o se (secondo la traccia interpretativa di Nietzsche e Freud) noi proiettiamo una coerenza sulle persone del mondo immaginandole come personaggio di una storia, ritagliando cioè narrazioni nella ricchezza confusa della vita. Qualunque senso scegliamo in questa analogia, ogni volta che vediamo qualcuno (una "persona o un "personaggio reale") agire in qualche modo, naturalmente interpretiamo la sua azione come la realizzazione di un progetto e gli attribuiamo un ruolo attanziale e un ruolo tematico. Se non riusciamo a farlo ci interroghiamo su "che cosa sta facendo", ma sempre gli attribuiamo la qualità della persona, cioè consideriamo sensate le sue azioni, cioè pensiamo che sotto ci sia un programma narrativo. È una considerazione che troviamo già negli stoici. Come scrive Epitteto (2019, p. 17), nella traduzione di Giacomo Leopardi:

Sovvengati che tu non sei qui altro che l'attore di un dramma, il quale sarà o breve o lungo a seconda della volontà del poeta. E se a costui piace che tu rappresenti la persona di un mendico, studia di rappresentartela acconciamente. Il simile se ti è assegnata la persona di uno zoppo, di un magistrato, di un uomo comune. Atteso che a te si spetta 
solamente di rappresentare bene quella qual si sia persona che ti è destinata; lo eleggerla si appartiene a un altro.

Vi è un profondo senso etico in questa attribuzione, che in qualche modo sembra generalizzare a tutta la vita il "principio di carità", cioè la massima che chiede all'interprete di attribuire il massimo di senso possibile a ogni atto linguistico o comunicativo. È il principio che Davidson (1991), Quine (1990), Wilson (1959) (ma forse il prototipo è una frase di Rabbi Meir, uno dei grandi maestri del Talmud, riportato in alcuni passi talmudici come Arachin 5a, commentato in Halbertal 1997) ritengono essere il fondamento dell'attività di interpretazione del linguaggio. Riconoscere gli altri come persone, cioè come personaggi di una storia di vita che perseguono progetti (che io possa capirli o meno) vuol dire distinguerli dalle cose del mondo, e riconoscere loro diritti, doveri, coscienza sia nel senso di autocomprensione che di intelligenza morale (Volli 2010). Ciò non è affatto banale, soprattutto se si estende, come di fatto accade, a tutti gli esseri umani, non solo a quelli di cui so abbastanza per immaginare dei programmi narrativi. Predicare di un essere umano la qualità della persona, la quale si associa naturalmente all'attribuzione di diritti e responsabilità, oltre che della continuità cognitiva, significa pensarla secondo le categorie dei mezzi e fini e farne un centro di imputazione di progetti. Per questa ragione gli esseri umani sono detti persone, ma lo sono anche le divinità (anche quelle della mitologia), gli angeli, gli esseri alieni dotati di coscienza (robot "intelligenti", extraterrestri, fiabe e streghe ecc.). A tutti costoro si possono attribuire più o meno gli altri termini della serie (una mente, un io, un'anima, uno spirito ecc.). Solo in certi casi usiamo attribuire tale carattere agli animali, quando cioè li vediamo come individui e attribuiamo loro un grado sufficiente di coscienza.

Per questa ragione la nozione di persona, che viene dalla finzione teatrale e narrativa, ci impone nel mondo morale un comportamento rispettoso della persona degli altri. Concetti etici come "dignità", "rispetto", "riconoscimento", sono correlati alla dimensione personale, così come la famosa formulazione dell'imperativo categorico di Kant - «Agisci in modo da trattare l'umanità, in te come negli altri, sempre come fine e mai come mezzo» (Die Metaphysik der Sitten [Fon- 
Di che cosa parliamo quando diciamo "persona"? damenti della metafisica dei costumi], (1797) sez. III) - sviluppa la sua attribuzione del carattere di persona alla continuità della coscienza. Discutere se vi sono soggetti unitari o meno, se la coscienza può essere riflessiva e se tale condizione garantisce continuità spaziale e temporale, è porsi non solo una questione teoretica, ma anche quella morale e politica della libertà e della responsabilità umana, il cui primo requisito è proprio che il soggetto sia una persona, l'agente vero delle sue azioni.

\section{Bibliografia}

Abbagnano N.

1971 Dizionario di filosofia, Utet, Torino.

Alberto Magno

2007 L'unità dell'intelletto (testo latino a fronte) [XIII sec.], Bompiani, Milano.

Ayto J.

1990 Word Origins, Black, London.

Bertagna M., Giuliani M.

2019 Bereshit, il Dizionarietto di ebraico, Morcelliana, Brescia.

Bertrand D.

2000 Précis de sémiotique littéraire, Nathan HER, Paris.

Bremmer J.N.

1987 The Early Greek Concept of the Soul. Princeton University Press, Princeton.

Buck C.D.

1949 A dictionary of selected synonyms in the principal indo-european languages, University of Chicago Press, Chicago.

Burke K.

1969 A Grammar of Motives, University of California Press, Berkeley.

de Libera A.

2007-08 Naissance du Sujet (Archéologie du Sujet I-II), Vrin, Paris.

Davidson D.

1991 "Three Varieties of Knowledge", in Subjective, Intersubjective, Objective, Clarendon Press, Oxford.

Dennett D.C.

1991 Consciousness Explained, Penguin, London.

Eco U.

1979 Lector in fabula, Bompiani, Milano.

1984 Semiotica e filosofia del linguaggio, Einaudi, Torino. 
Edelman G.M.

1989 The Remembered Present: A Biological Theory of Consciousness, Basic Books, New York.

Ellis B.

2001 Scientific essentialism, Cambridge University Press, Cambridge.

Elster J.

1985 The multiple Self, Cambridge University Press, Cambridge.

Epitteto

2019 Manuale, trad. di Giacomo Leopardi, Pimiceri Editore, Padova.

Eugeni R.

1999 Analisi semiotica dell'immagine, Educatt, Milano.

Franzini E.

2012 Introduzione alla fenomenologia, SHAKE, Milano.

Freeman M.

1993 Rewriting the self, Routledge, London - New York.

Freud S.

1923 Das Ich und das Es, Internationaler Psychoanalytischer Verlag, Leipzig.

Goldman A.I.

2015 Theory of Human Action, Princeton University Press, Princeton.

Halbertal M.

1997 People of the Book, Harvard University Press, Boston.

Handke P.

1969 Die Innenwelt der Außenwelt der Innenwelt, Suhrkamp.

Hofstadter D.R., Dennett D.C.

1981 The Mind's I, Basic Books, New York - Berlin.

Hume D.

1739 A Treatise of Human Nature, London, Noon.

Jackendorff R.

1990 Semantic Structures, MIT Press, Cambridge (Mass.).

1991 Parts and boundaries, «Cognition», 41, pp. 9-45.

Kripke $\mathrm{S}$.

1980 Naming and Necessity, Basil Blackwell, Oxford.

Lyons W.

1986 The Disappearance of Introspection, Cambridge (Mass.): MIT Press.

Lakoff G., Johnson M.

1980 Metaphors we live by, Chicago University Press, Chicago. 
Di che cosa parliamo quando diciamo "persona"?
Locke, J.

1690 An Essay Concerning Human Understanding, ora in https://www.gutenberg.org/cache/epub/10615/pg 10615.html.

Lotman J.M., Uspenskij B.A.

1973 Tipologia della cultura, Bompiani, Milano.

Mauss M.

1938 Une catégorie de l'esprit humain: la notion de personne celle de 'moi', "Journal of the Royal Anthropological Institute”, vol. LXVIII (Huxley Memorial Lecture; ora: https://psychaanalyse.com/pdf/une_categorie_de_1_ esprit_humain_la_notion_de_personne_celle_de_ moi_marcel_mauss.pdf).

Pinker S.

2007 The Stuff of Thought: Language As a Window Into Human Nature, Penguin, London.

Putnam H.

1975 “The meaning of 'Meaning', in K. Gunderson (a cura di), Language, mind and knowledge, University of Minnesota Press, Minneapolis 1975, pp. 131-193 .

Ricœur P.

1990 Soi-même comme un autre, Le Seuil, Paris.

1960 Word and Object, MIT Press, Cambridge (Mass.).

Ryle G.

1965 The Concept of Mind, Barnes \& Noble, New York.

Saussure F. de

1916 Cours de linguistique générale, Payot, Lausanne-Paris (trad. it. Corso di linguistica generale, Laterza, RomaBari 1967).

Searle J.R, Ferraris M.

2018 Il denaro e i suoi inganni, Einaudi, Torino.

Snell B.

1946 Die Entdeckung des Geistes. Studien zur Entstehung des europäischen Denkens bei den Griechen, Claassen \& Goverts, Hamburg.

Sparti D.

1996 Soggetti al tempo, Feltrinelli, Milano.

Spaemann R.

1996 Personen, Cotta, Stuttgart.

Taylor Ch.

1989 Sources of the Self: The Making of Modern Identity, Harvard University Press, Cambridge (Mass.). 
Tommaso d'Aquino

1959 In Aristotelis librum de anima commentarium [XIII sec.], Marietti, Torino.

Volli U.

2008 Lezioni di filosofia della comunicazione, Laterza, RomaBari.

2010 Al di là dell'essenza, il linguaggio, «Quaderni laici», 1.

Wiley N.

1994 The semiotic self, University of Chicago Press, Chicago. Wilson N.

1959 Substances without Substrata, «The Review of Metaphysics», 12, n. 4.

Whorf B.L.

1945 Grammatical Categories, «Language», 21 (1). 
L'individuo moderno e il suo Altro... o no?

Un ritorno critico sull'antropologia della persona

Carlo Capello

L'antropologia della persona, di cui mi occuperò in questo breve saggio, sembra avere una data di nascita precisa: il 1938, l'anno in cui Marcel Mauss pubblicò il saggio Una categoria dello spirito umano: la nozione di persona, quella di "io". Tornerò tra breve in modo più esteso sulle tesi dell'opera di Mauss, nella quale l'antropologo francese cerca di tratteggiare l'evoluzione storica delle idee di persona, sé e individuo. Prima, però, vorrei soffermarmi sulle sue dimensioni etiche e politiche, esplicitate nelle conclusioni: "Chi può sapere, inoltre, se questa "categoria" [la nozione di io e di individuo, $N d A$ ], che tutti riteniamo fondata, sarà sempre riconosciuta come tale? Essa ha una forma e un fondamento solo per noi, presso di noi. Perfino la sua forza morale - il carattere sacro della persona umana - è posta in discussione non soltanto in tutto l'oriente, dove non si è mai pervenuti alle nostre conoscenze, ma anche in paesi in cui tale principio è stato scoperto. Noi abbiamo grandi beni da difendere, con noi può scomparire l'Idea» (Mauss 1991, p. 381).

Due punti nel saggio di Mauss meritano di essere sottolineati. Il primo è l'automatica associazione da lui tracciata tra l'idea di persona come soggetto autonomo e sacro e la 
modernità - con tutte le implicazioni etnocentriche che tale associazione, data un po' per scontata, può comportare. Il secondo ma non meno importante punto ha a che fare con le motivazioni morali dell'intervento di Mauss, che risaltano particolarmente nell'accorato appello sopra citato. Se teniamo a mente gli anni in cui è stato scritto e pubblicato il saggio, è chiaro che l'antropologo si riferisce nel finale al regresso barbarico dei regimi totalitari, denunciati, da questo studioso socialista e umanista, come annichilimento della persona umana. Tuttavia, questa dimensione morale e politica del suo lavoro, così come la critica dell'egemonia della razionalità strumentale e del mercato nel suo saggio sul dono, non sono del tutto sviluppate da Mauss, rimanendo in larga misura implicite o tutt'al più confinate nelle conclusioni di saggi che si volevano innanzitutto scientifici e distaccati. Ciò è dovuto a quel "patto empirista e primitivista" (Mazzarella 2017) che ha caratterizzato buona parte dell'antropologia culturale del Novecento, limitandone la possibilità di tradursi pienamente in una vera critica culturale (Marcus, Fischer 1994). Tuttavia, è indubbio che il saggio di Mauss sia un lavoro importante, ambizioso e influente, per quanto non privo di limiti. In questo mio intervento, partirò da alcuni dei temi già presenti in quel pionieristico lavoro per riflettere, attraverso una rilettura dell'antropologia della persona, sulla nozione di individuo e le possibilità di un suo superamento.

\section{Primo movimento}

Uno dei motivi per cui ho iniziato la mia riflessione partendo da Mauss è che l'antropologia della persona non è, in realtà, un campo di studi ben definito - come l'antropologia medica o l'etnopsichiatria, per esempio. Al contrario, è un ambito molto eterogeneo di ricerche e riflessioni unite meno dal comune oggetto di studio che dal riferimento ad alcuni antenati totemici, come Mauss, appunto, o Maurice Leenhardt (1947) o ancora, per citare studiosi più recenti, Louis Dumont (1989) e Marilyn Strathern (1988). Nonostante l'evidente importanza del tema - le nozioni culturali di io e di persona - gli antropologi non sono, infatti, veramente riusciti a dare coerenza e vigore intellettuale a questo campo di studi (Capello 2016).

Anche per questo motivo, la cosa migliore è definire l'antropologia della persona in modo minimale, come lo 
L'individuo moderno e il suo Altro...

o no?

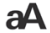

studio etnografico e comparativo delle concezioni culturali del soggetto umano ${ }^{1}$. La principale domanda di ricerca di questo variegato ambito di studi è chiaramente espressa dal titolo di un importante articolo di Shweder e Bourne (1991): "Il concetto di persona varia a seconda delle culture?". Una domanda che può facilmente convertirsi in quella formulata da Jean e John Comaroff (2019) in un notevole saggio sul quale ritorneremo più avanti, $L a$ persona. Una prospettiva africana: "Il concetto di soggetto autonomo è una creazione europea?". O ancora: l'individuo autonomo è un'invenzione o una scoperta moderna, come pensava Mauss?

Sono tali questioni ad aver delimitato l'ambito e ad aver stimolato l'emergere dell'antropologia della persona, nata dal riconoscimento etnografico della pluralità culturale delle rappresentazioni del soggetto umano e dalla comparazione di questa pluralità con il concetto moderno ed europeo di individuo.

La comparazione, implicita o esplicita, tra le differenti concezioni di persona e la concezione occidentale e moderna - l'idea di individuo - che caratterizza il campo di ricerca, ne rappresenta a un tempo il punto di forza e il suo principale limite. È potenzialmente un punto di forza perché la comparazione può aiutarci a decostruire l'illusoria fatticità dei concetti e delle idee occidentali, che tendiamo a dare per scontati. Allo stesso tempo però, in questo confronto interculturale si corre sempre il rischio dell'etnocentrismo, come accade, ahimè, addirittura a un padre fondatore come Mauss. Poiché la nozione di persona individuale ci è così cara, per via della valorizzazione del sé, della libertà, dei diritti individuali, dell'interesse personale che sembra portare necessariamente con sé, siamo portati infatti a "giudicare" le concezioni culturali diverse dalla nostra, anziché descriverle e comprenderle nella loro autonomia e pluralità. I limiti della comparazione sono poi accentuati dall'etnocentrismo implicito della nostra lingua: nell'uso quotidiano, infatti, termini come individuo, persona, sé sono usati più o meno come sinonimi, rendendo ancora più difficile delineare

1. O meglio, dei soggetti umani e non umani, dato che le concezioni culturali fanno spesso riferimento anche a persone non-umane. Su questo tema, mi permetto di rimandare al cap. 3 del mio Antropologia della persona (Capello 2016). 
e dar senso a rappresentazioni culturali in cui la persona non è vista come un sé o come un individuo autonomo e indipendente.

Inoltre, nell'antropologia della persona è spesso presente un altro limite non da poco - e non facilmente risolvibile. Poiché il nostro compito è osservare e comprendere i differenti modi in cui i soggetti umani sono culturalmente costruiti (nel senso, a un tempo, di rappresentati e modellati culturalmente), una domanda etico-filosofica soggiace sempre alle nostre indagini: al di là dell'eterogeneità culturale, che cos'è una persona? Cos'è persona? Che cosa è il soggetto? Che cosa è l'umano? La domanda è sempre lì, eppure gli antropologi culturali - a causa del già menzionato patto empirista alle origini della disciplina, il suo volontario e deciso allontanarsi da ogni forma di speculazione filosofica - normalmente preferiscono aggirare questo genere di questioni ontologiche, dedicandosi quasi esclusivamente alla descrizione dei significati e dei saperi locali ${ }^{2}$. A mio parere, però, poiché la domanda va affrontata, lo studio antropologico della persona può svilupparsi pienamente solo a patto di dar vita a un vero dialogo con la filosofia, in particolare con la teoria critica e le varie teorie dell'intersoggettività e del transindividuale, come vedremo nelle conclusioni.

\section{Persone}

Che l'argomentazione di Mauss sia segnata da un certo etnocentrismo, dovuto all'accettazione implicita di una specifica visione del soggetto, tipicamente moderna, è piuttosto evidente leggendo il suo saggio. Mentre riguardo a temi come lo scambio e l'economia, il suo approccio comparativo lo conduce a mettere in dubbio l'ideologia universalista occidentale (Mauss 2002), l'antropologo francese non riesce a fare lo stesso nei confronti del concetto di persona: l'individuo autonomo continua a rimanere il suo riferimento morale ultimo. Nella sua ricostruzione dell'idea di persona, infatti, Mauss segue una logica evoluzionistica che, per quanto ancora mol-

2. Le eccezioni, ovviamente, non mancano: oltre a Remotti (2019) e alla sua proposta concettuale di "condividuo", si veda l'interessante articolo di Maurice Bloch (2011), The $B l o b$, in cui l'autore intreccia antropologia culturale e scienze cognitive per arrivare a una definizione del sé e della soggettività. 
L'individuo moderno e il suo Altro...

o no?

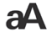

to diffusa nell'opinione pubblica, già nel 1938 suonava piuttosto invecchiata in antropologia culturale. Finisce così per presentare la nozione moderna di individuo come il vertice di un progressivo sviluppo delle idee di persona, condannando implicitamente le rappresentazioni altre, non occidentali e non-moderne di soggetto, come arretrate e imperfette. Mauss inizia il suo saggio affermando che la percezione del proprio sé, della propria unicità, è un'esperienza universale, propria di ogni essere umano. Tuttavia, continua, il riconoscimento e la valorizzazione sociale dell'individualità non lo sono affatto. La rappresentazione collettiva della persona, a suo dire, si sarebbe evoluta passando attraverso diverse fasi: dalle "società arcaiche", dove l'individuo autonomo è negato a favore del collettivo - il clan e la tribù - si arriverebbe, progressivamente, alla società e al pensiero moderni, dove invece il valore del sé individuale è pienamente riconosciuto. Lo studioso riassume la sua posizione così: «Da un semplice mascheramento alla maschera, da un personaggio a una persona, a un nome, a un individuo... il percorso è compiuto» (Mauss 1991, p. 381).

Mauss non vuole e non può mettere in discussione la concezione moderna di persona in quanto individuo autonomo e indipendente per motivi ideologici e morali, per via del suo umanismo. Cosicché, la sua esplorazione si traduce in un'ulteriore versione, un po' più articolata, della diffusa idea che contrappone le società tradizionali e arcaiche alla modernità. Una frattura, una contrapposizione noi/altri, moderni/non moderni, che corrisponderebbe al passaggio dal non riconoscimento dell'individualità - ridotta a un personaggio, a un ruolo, a una maschera sociale - alla scoperta e all'emancipazione dell'io, in quanto persona individuale. Si tratta, possiamo chiaramente dire, di un'idea fortemente connotata sul piano ideologico, funzionale all'autorappresentazione celebrativa della modernità occidentale e alla giustificazione della sua egemonia. Neppure Mauss, nonostante la sua apertura di pensiero e il suo genio antropologico, è riuscito a svincolarsi da certe visioni ideologiche rispetto alla persona e all'io.

È questo, invece, ciò che prova a fare Louis Dumont. Quest'ultimo, che di Marcel Mauss fu allievo, ne riprende i suggerimenti sulla relatività delle idee di persona per portarli alle loro estreme conseguenze e, in questo modo, rove- 
sciarli. Dumont (1989), che ha elaborato la sua antropologia della persona a partire dalle sue ricerche sulla religione e il sistema castale indiano, afferma infatti che l'individualismo, l'idea stessa di individuo, è il primo ostacolo da superare per comprendere pienamente le nozioni non-occidentali di persona. Da questo punto di partenza epistemologico radicale, Dumont sviluppa un modello che contrappone ancora una volta le concezioni moderne e quelle non-moderne, noi e gli altri, ma in un certo senso cambiandone il senso e il valore, da un punto di vista antropologico. Egli articola esplicitamente la differenza tra società moderne e società non-moderne nei termini delle differenti concezioni del soggetto: la persona individuale, l'individuo proprio delle società moderne si contrappone così alla persona sociocentrica e alle concezioni olistiche proprie delle culture nonmoderne. Si delineano così due linee distinte di idee-valori, di ideologie: da un lato un'ideologia "olistica", tipica delle culture tradizionali - come quella induista che, a suo parere, ne rappresenta la manifestazione massima - che enfatizza la società in quanto intero, e vede nel singolo solo una parte di quest'ultima; dall'altra l'ideologia moderna, che al contrario dà valore esclusivamente all'individuo, negando realtà ontologica alla società. La concezione sociocentrica di persona e quella individualistica sono, in quest'ottica, totalmente differenti e opposte, irriducibili l'una all'altra.

Le tesi di Dumont, per quanto anche molto criticate, hanno avuto una notevole influenza nell'antropologia della persona, conducendo il dibattito a concentrarsi a lungo sull'opposizione tra concezioni sociocentriche e concezioni individualistiche (Capello 2016). Due punti del discorso di Dumont vanno ancora evidenziati per la nostra argomentazione. In primo luogo, per quanto opposte, le due concezioni di persona non sono simmetriche, dal suo punto di vista. Lideologia moderna di persona ha al suo centro la contrapposizione tra individuo e società, mentre nell'ideologia olistica non è così, perché il soggetto è ricompreso all'interno della totalità sociale. Tuttavia, ancora più interessante è il fatto che la posizione di Dumont consiste in una critica netta del senso comune moderno, perché l'individualismo è, per lui, solo una "falsa coscienza", mentre è la concezione tradizionale, olistica a rivelarsi più realistica dal punto di vista sociologico: «una società, come quella concepita dall'indivi- 
L'individuo moderno e il suo Altro...

o no?

dualismo - scrive Dumont - non è mai esistita [...] perché l'uomo vive di idee sociali» (Dumont 1989, p. 81).

\section{Oltre l'individuo}

Da Mauss in poi, e soprattutto grazie al decisivo contributo di Dumont, l'antropologia della persona ha dunque assunto per molto tempo un approccio dualistico, incentrato sulla contrapposizione "individualismo vs. sociocentrismo". Un approccio che ha trovato la sua espressione più chiara nelle indagini di Shweder e Bourne (1991), che riprendono direttamente il paradigma di Dumont e Mauss. Nonostante l'importanza per il campo di studi, in questo lavoro l'approccio dualistico mostra tutti i suoi limiti. In effetti, per quanto utile come prima sistematizzazione del materiale etnografico e come punto di partenza teorico, la contrapposizione tra individuo e persona sociocentrica non è realmente comparativa, perché finisce per ridurre tutte le molteplici, differenti forme di persona presenti nelle diverse culture a due grandi e semplificanti ideal-tipi. In particolare, come vedremo, la macro-categoria del sociocentrismo non è sufficiente per comprendere i diversi tipi di persona duale, dividuale, relazionale che possiamo ritrovare nell'archivio etnografico globale. Inoltre, nonostante tutto, nella logica dell'opposizione dicotomica la persona individuale rimane ancora il metro di giudizio antropologico con cui misurare le altre concezioni culturali - altre, appunto, in opposizione alla nostra, al primato del sé e dell'io. In altre parole, l'opposizione dualistica noi/altri, pre-moderni/moderni può essere, nella sua semplicità, sviante.

Tutt'al più, come afferma Marilyn Strathern (1988), una tale opposizione può avere giusto un valore euristico, configurandosi come una "finzione" teorica, utile per evidenziare la differenza culturale, ma che va a sua volta decostruita e superata. È questo il percorso intrapreso dalla stessa Strathern nel suo capolavoro, The Gender of the Gift, per esplorare il pensiero melanesiano riguardo alla persona, al sesso, al genere, alla riproduzione e al dono. Prendendo come punto di partenza il seminale lavoro di Maurice Leenhardt (1947) tra i Kanak della Nuova Caledonia dedicato a indagare la nozione locale di persona - il "kamo" - l'antropologa britannica mostra con eccezionale efficacia come nelle culture melanesiane la persona sia vista 
non come un sé, come un'individualità atomistica, bensì come un "insieme di relazioni sociali". Anziché un individuo, troviamo qui una persona dividuale: il dividuo. Il soggetto è un intreccio di relazioni con gli altri, un nodo che, nella sua singolarità, è composto di tutti i legami - parentali, politici, economici... - con altri significativi. Il dividuo è fatto di altri e di relazioni con gli altri. Se il soggetto occidentalemoderno è costruito come un individuo-monade, come un atomo separato dagli altri atomi, e visto come il fondamento ultimo della realtà sociale ma opposto ad essa, il dividuo melanesiano non è separato dagli altri, ma fatto di relazioni, e non è posto in contrasto con la società. Inoltre, la persona melanesiana è "divisibile", perché le relazioni che costituiscono il dividuo possono staccarsi per produrre nuove relazioni, come avviene nell'unione sessuale riproduttiva o nell'incarnazione dei legami sociali in oggetti cerimoniali, sotto forma di doni. Nello scambio di doni, il donatore stacca una parte di sé - una relazione sociale - per "dar vita" al bene donato creando o sostenendo la relazione sociale tra donatore e donatario. Il che spiega perché i doni non sono "cose", nella filosofia indigena, semplici oggetti, bensì persone, essendo composte anch'esse di relazioni sociali proprio come le persone umane ${ }^{3}$.

Il dividuo è il soggetto del dono, l'attore di un'economia del dono basata sulla logica dello scambio per unire le persone costruendo relazioni sociali, proprio come la persona individuale è il soggetto dell'economia di mercato, afferma Strathern (1988). Così, proprio come nella società di mercato abbiamo soggetti reificati, persone mercificate e merci feticizzate, nelle culture dividuali abbiamo oggetti personificati - i doni - e persone divisibili, composte di rapporti sociali. Ma il diacritico della dividualità si trova, innanzitutto, nella relazione alla società. Il concetto di individuo implica una separazione, uno iato incolmabile tra il sé e la società, mentre le persone dividuali sono sociali in sé e per sé, la società è incorporata in loro. Anche se, per essere più precisi, chiarisce Marilyn Strathern, in queste culture dividuali, così come non ci sono individui, non vi è neppure la

3. Per un'esposizione a un tempo completa e criticamente fondata delle teorie antropologiche del dono, si veda il libro di Matteo Aria (2016), che dà ampio spazio alla questione cruciale dell'intreccio tra persone e cose nelle culture del dono. 
L'individuo moderno e il suo Altro...

o no? "società". Non ci sono monadi, né entità collettive reificate. Solo relazioni sociali.

\section{Ancora oltre}

Per Strathern (1988), come si è detto, la contrapposizione noi/altri è solo una consapevole finzione metodologica, analoga alla distinzione dono/merce cui è strettamente connessa (Aria 2016; Gregory 2012). In ogni caso, l'analisi antropologica deve procedere oltre questa dicotomia, decostruendola per evitare facili semplificazioni e opposizioni ingannevoli. Anche nello studio antropologico della persona è necessario un approccio dialettico: alla tesi dell'universalità dell'individuo va contrapposta in prima battuta l'antitesi della relatività della persona. Ma, per cogliere le idee di persona nella loro complessità, da qui bisogna fare un passo oltre per arrivare alla sintesi (Capello 2013, 2016).

Un approccio dialettico al tema della persona si ritrova nel saggio già citato di Jean e John Comaroff (2019), basato sulla loro esperienza etnografica tra gli Tswana dell'Africa del Sud. Sebbene, rilevano i due autori, parlare di una concezione Tswana di persona tradizionale - come di qualcosa di fuori dal tempo, di un'antropologia locale immobile e immutabile - sia solo un'astrazione, nel sistema di significato locale troviamo delle rappresentazioni del soggetto che sfidano ogni semplice categorizzazione binaria. La persona Tswana, espressione di una società patrilineare e patriarcale, era concepita come relazionale e sociocentrica, per usare i concetti classici dell'antropologia della persona. Il soggetto era visto come costituito delle relazioni sociali, in particolare dai rapporti di parentela e di alleanza, che lo uniscono agli altri soggetti all'interno del gruppo. In questo senso, possiamo leggere la concezione Tswana come un esempio della più vasta concezione africana di persona (Riesman 1986), una rappresentazione del soggetto che enfatizza il legame sociale e l'appartenenza al gruppo e alla comunità. Tuttavia, non è questo che i due antropologi sudafricani intendono sottolineare, evidenziando piuttosto il fatto che la concezione Tswana di persona ha una evidente dimensione processuale e costruttivista. La condizione di persona non è qualcosa di dato una volta e per tutte. Richiede uno sforzo continuo, un costante lavoro personale di costruzione del sé e delle relazioni sociali che lo sostengono, del sé come 
relazioni sociali. La persona è il prodotto di ciò che in lingua Tswana è definito itirela, lavoro su di sé.

Focalizzandosi su questo aspetto della nozione locale di soggetto come autocostruzione continua, Jean e John Comaroff (2019) intendono mostrare l'affinità tra la persona Tswana e la sua controparte occidentale moderna e post-moderna. La persona Tswana non è solo sempre riconosciuta nella sua unicità relazionale, è una sorta di "selfmade man". E se itirela ricorda da vicino l'etica e la politica della cura di sé immaginata da Foucault (2014), la persona locale non è poi molto distante dal modello post-moderno e neo-liberale dell' "imprenditore di sé" (Comaroff e Comaroff 2019). L'esempio Tswana ci ricorda allora che i concetti locali di persona possono insistere tanto sulle qualità relazionali del soggetto quanto su quelle più "individualistiche", entrambe presenti in ogni nozione di persona, sebbene con importanza e peso differente a seconda delle diverse culture, epoche e circostanze sociali e storiche (Morris 1994; Remotti 2009).

E questo è vero non solo per le concezioni Tswana, ma anche per le concezioni occidentali, nonostante e al di là di ogni individualismo. Mostrare, come fanno i Comaroff, che le nozioni native e locali di persona non sono così diverse da quelle moderne non è infatti che il primo passo verso la sintesi dialettica, il primo passo per superare l'implicito dualismo tra modernità e non modernità nel campo delle idee di soggetto e di io. Il passo successivo consiste nell'investigare etnograficamente le rappresentazioni proprie dell'Occidente contemporaneo, come hanno provato a fare Janet Carsten (2004) e Marshall Sahlins (2014) nelle loro indagini sugli stretti nessi simbolici tra parentela e persona. Janet Carsten ci rammenta che la classica nozione di persona come individuo - come soggetto autonomo, separato e distinto dalle connessioni sociali e dal sociale - è in larga misura un costrutto filosofico e giuridico. Nella reale vita sociale di tutti i giorni troviamo invece all'opera concezioni di persona differenti, che presentano in verità molte dimensioni e sfumature relazionali, sociocentriche e dividuali. Queste concezioni di persona, per quanto nascoste e schiacciate dalla dominante ideologia individualistica, emergono con chiarezza in relazione alla sfera della famiglia e delle relazioni primarie. Anche nella cultura occidentale contemporanea, 
L'individuo moderno e il suo Altro...

o no?

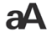

nonostante il pervasivo individualismo, i parenti e gli amici intimi «condividono la vita l'uno dell'altro», secondo la bella espressione di Sahlins (2014). In altre parole, una concezione relazionale e dividuale di persona si delinea in relazione alla realtà parentale e amicale, sebbene altre sfere della nostra esperienza sociale - in particolare quella economico-lavorativa - siano dominate da rappresentazioni individualistiche e individualizzanti. In realtà, come mostra anche James Carrier (1992), non vi è un'unica persona moderna: differenti e anche conflittuali concezioni di persona sono presenti nel sistema simbolico contemporaneo.

Come ho mostrato nella mia più ampia indagine sull' $A n$ tropologia della persona (Capello 2016), queste molteplici concezioni sono espressione delle diverse aree in cui possiamo suddividere la vita sociale moderna: la vita quotidiana, la vita familiare e domestica, le istituzioni politiche ecc. E tuttavia, a causa della colonizzazione della forma-di-vita quotidiana da parte dell'economia di mercato, l'individualismo - primariamente nella forma del modello antropologico dell'homo oeconomicus - ha una posizione di assoluta egemonia che non può non influenzare a livello simbolico la più generale concezione del soggetto e dell'io. Indagare le concezioni di persona moderne e contemporanee conduce dunque a porsi anche la seguente domanda: cosa significa il dominio del capitalismo e del mercato per l'idea di persona?

La risposta non è così immediata come la mia precedente enfasi sull'individualismo potrebbe far pensare. Nella sua originale indagine antropologica sul mercato finanziario, Scommettere sulle parole, Arjun Appadurai (2016) espone tutta una serie di dubbi e timori rispetto al destino dell'individuo nel mondo contemporaneo che ricordano da vicino l'accorato appello di Mauss ${ }^{4}$. Secondo Appadurai, l'egemonia della finanza speculativa all'interno delle logiche sociali del tardo capitalismo non implicherebbe una crescita dell'individualismo, nel senso comune del termine. Al contrario, implicherebbe la crisi del soggetto individuale, una crisi che porterebbe a un "ritorno della persona dividuale". Il soggetto contemporaneo sarebbe, infatti, sottoposto a processi di decostruzione che lo disassemblano in parti che

4. Per una lettura delle tesi di Appadurai nell'ottica dell'antropologia della persona si veda Remotti (2019). 
vanno oltre la soggettività, come dimostra per esempio la questione del "capitale umano", delle risorse economiche, culturali e relazionali con le quali la persona è oggi giudicata e messa all'opera. Appadurai (2016) parla, a questo riguardo, di "dividualità predatoria". Il soggetto dividuale non-moderno, espressione dell'economia del dono, sembra tornare nel tardo capitalismo finanziario sotto forma di spettro nel momento in cui le relazioni sociali diventano esse stesse un asset di investimento. Il valore dell'individuo moderno è a rischio, afferma Appadurai, ma la soluzione non può consistere un ritorno al vecchio mito liberale che si alimentava della nozione di autonomia individuale, bensì nella capacità di cogliere la potenzialità emancipatrici della crisi del soggetto contemporaneo nascoste nell'idea stessa di dividuo.

Le affermazioni di Appadurai sono al contempo problematiche e suggestive. Nonostante tutto, non ritengo che una dividualità predatoria abbia preso il posto del vecchio individuo autonomo liberale come modello antropologico dominante. In modo meno originale, ma forse meno astrattamente speculativo, penso che il neo-soggetto del tardo-capitalismo porti ai suoi estremi l'egocentrismo moderno (Dardot, Laval 2013). D'altra parte, l'idea di una dividualità emancipatrice è piuttosto affascinante. Come possiamo pensarla?

\section{Dal dividuo al transindividuale}

Nei miei lavori precedenti (Capello 2013, 2016) ho suggerito che una possibile risposta si possa trovare nelle numerose e profonde affinità tra le concezioni sociocentriche e relazionali di persona e alcuni momenti della filosofia occidentale che hanno pensato l'umano al di là delle strettoie dell'individualismo astratto. In particolare, si può notare una suggestiva affinità tra il dividuo melanesiano descritto da Strathern (1988) - che, come si è visto, è pensato e vissuto come un insieme, un nodo di relazioni sociali - e la teoria del transindividuale (Bartsidis 2010; Morfino 2008, 2010). Il concetto di transindividuale è stato coniato da Gilbert Simondon (2001) e in seguito ripreso e sviluppato da Etienne Balibar per rileggere il pensiero di Spinoza (Balibar 2002) e illuminare di nuova luce l'antropologia di Marx (Balibar 1994). Quest'ultima è stata spesso identificata come una concezione sociocentrica e collettivistica, non dissimile 
L'individuo moderno e il suo Altro...

o no?

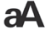

dalla persona olistica non-moderna delineata da Dumont in opposizione all'individualismo liberale, ma è un errore. In realtà, mostra bene Balibar (1994), l'antropologia di Marx è "transindividuale", come si vede leggendo attentamente la VI Tesi su Feuerbach: «Nella sua realtà [l'essenza umana] è l'insieme dei rapporti sociali» (Marx 1969, p. 84). Qui, con un duplice passaggio dialettico, Marx rifiuta ogni lettura individualistica ed essenzialistica dell'umano, riconducendo tanto il singolo quanto l'essere-specie ai rapporti sociali che vanno oltre l'individuo nel momento in cui lo formano e costituiscono per ciò che è. In questo modo, il transindividualismo marxiano riesce a superare l'opposizione di senso comune tra individuo e società, che così fortemente limita le nostre stesse riflessioni sul soggetto e sul sociale. Scrive Marx (1977, p. 228) nei suoi Manoscritti economicofilosofici del 1844: «... come la società stessa produce l'uomo in quanto uomo, così essa è prodotta da lui [...] è da evitare innanzitutto di fissare ancora la società come un'astrazione di fronte all'individuo. L'individuo è un ente sociale.» Se sovrapponiamo le due affermazioni di Marx, si delinea chiaramente un rifiuto di ogni ipostatizzazione del sociale e di ogni idealizzazione dell'individuo, ricondotti alla comune matrice di rapporti sociali - analogamente a quanto affermato da Strathern (1988) a partire dalle sue ricerche melanesiane. Laffinità tra la concezione transindividuale evocata da Marx e rimessa in luce da Balibar da un lato, e le concezioni dividuali di persona dall'altro, il dividuo, è chiara e merita ulteriori riflessioni e sviluppi teorici.

Una vera antropologia della persona dovrebbe, credo, partire da qua: valorizzare le concezioni native, non-moderne di soggetto in tutta la loro profondità speculativa ${ }^{5}$ facendole dialogare con le strade aperte dal pensiero critico-filosofico occidentale ${ }^{6}$. Solo una prospettiva di questo genere può darci la possibilità di andare oltre le false alternative, proprie di molta parte del pensiero moderno, tra

5. Come suggerito in modo molto efficace da Viveiros de Castro (2017), quando afferma la necessità di trattare le nozioni e le concezioni proprie delle altre culture con la stessa serietà con cui discutiamo le filosofie occidentali.

6. Partendo da assunti analoghi, recentemente Francesco Remotti (2019) ha intrecciato antropologia e filosofia per elaborare il concetto di "condividuo" al fine di superare i limiti e i paradossi del pensiero individualista ed essenzializzante. 
autonomia e socialità, tra libertà e legami sociali, tra società moderne e non moderne.

\section{Bibliografia}

Appadurai A.

2016 Scommettere sulle parole. Il cedimento del linguaggio nell'epoca della finanza derivata, Raffaello Cortina, Milano.

Aria M.

2016 I doni di Mauss. Percorsi di antropologia economica, CISU, Roma.

Balibar E.

1994 La filosofia di Marx, Manifestolibri, Roma.

2002 Spinoza. Il transindividuale, Ghibli, Milano.

Bartsidis M.

2010 Sul concetto di transindividualità e alterità in Balibar, in N. Marcucci, L. Pinzolo (a cura di), Strategie della relazione, Meltemi, Roma.

Bloch M.

2011 The Blob, «Anthropology Of This Century», n. 1 (May). Capello C.

2013 Dai Kanak a Marx e ritorno. Antropologia della persona e transindividuale, "Dada. Rivista di antropologia postglobale», n. 1, pp. 99-114.

2016 Antropologia della persona. Un'esplorazione, FrancoAngeli, Milano.

Carrier J.

1992 Emerging Alienation in Production. A Maussian History, «Man», XXVII, n. 3, pp. 539-558.

\section{Carsten J.}

2004 The Person, in Ead., After Kinship, Cambridge University Press, Cambridge.

Comaroff J., Comaroff J. L.

2019 Teoria dal Sud del Mondo, Rosenberg \& Sellier, Torino.

Dardot P., Laval C.

2013 La nuova ragione del mondo. Critica della razionalità neoliberista, DeriveApprodi, Roma.

Dumont L.

1989 Homo hierarchicus, Adelphi, Milano.

Foucault M.

2014 La cura di sé. Storia della sessualità 3, Feltrinelli, Milano.

Gregory C.

2016 Gifts and Commodities, Hau Books, Chicago/London. 
L'individuo moderno e il suo Altro...

o no?
Leenhardt M.

1947 Do kamo. La personne et le mythe dans le monde mélanésien, Gallimard, Paris.

Marcus G., Fischer M.J.

1994 Antropologia come critica culturale, Anabasi, Milano.

Marx K.

1977 Opere filosofiche giovanili, Editori Riuniti, Roma.

1969 Tesi su Feuerbach, in appendice a F. Engels, Ludwig Feuerbach e il punto di approdo della filosofia classica tedesca, Editori Riuniti, Roma.

Mauss M.

1991 "Una categoria dello spirito umano: la nozione di persona, quella di 'io'”, in Id., Teoria generale della magia e altri saggi, Einaudi, Torino.

2002 Saggio sul dono, Einaudi, Torino.

Mazzarella W.

2017 The Mana of Mass Society, The University of Chicago Press, Chicago.

Morfino V.

2008 Simondon e il transindividuale, «Il Protagora», XXXVI, n. 12, pp. 395-400.

2010 Transindividuale e/o riconoscimento: ancora sull'alternativa Hegel/Spinoza, in N. Marcucci, L. Pinzolo (a cura di), Strategie della relazione, Meltemi, Roma.

Morris B.

1994 Anthropology of the Self, Pluto Press, London.

Remotti F.

2009 Antropologia della persona, in Id., Noi, primitivi. Lo specchio dell'antropologia, Bollati Boringhieri, Torino.

2019 Somiglianze. Una via per la convivenza, Laterza, RomaBari.

Riesman P.

1986 The Person and Life-Cycle in African Social Life and Thought, «African Studies Review», XXIX, n. 2, pp. 71138.

Sahlins M.

2014 La parentela. Cos'è e cosa non è, Elèuthera, Milano.

Shweder R., Bourne E.

1991 Il concetto di persona varia a seconda delle culture?, in $\mathrm{R}$. Shweder, R. Le Vine (a cura di), Mente, sé, emozioni, Argo, Lecce.

Simondon G.

2001 Lindividuazione psichica e collettiva, DeriveApprodi, Roma. 
Carlo Capello
Strathern M.

1988 The Gender of the Gift, University of California Press, Berkeley.

Viveiros de Castro E.

2017 Metafisiche cannibali, Ombre Corte, Verona. 
'Persona' è l'inverso di individuo? Qualche riflessione a partire da Peirce Emanuele Fadda
Im Kampf zwischen Dir und der Welt, sekundiere der Welt.

Franz Kafka (1918)

In questo contributo vorrei provare a pensare la nozione e il tema della persona a partire dalla riflessione di Charles $\mathrm{S}$. Peirce. Laspetto più importante del concetto peirceano di personalità è lo sganciamento dall'individualità: una persona non è (e non può essere mai ridotta a) un individuo. Io proporrò qui una versione radicale di questa idea: persona è $i l$ contrario di individuo, e un ente è tanto più personale quanto meno è individuale.

Una tale concezione, se ci si limitasse a enunciarla in questi termini, nonostante la sua paradossalità e la sua radicalità, non sarebbe in fondo troppo lontana da molte altre forme di pensiero del genere, che "risolvono" il ruolo dell'individuo nel suo contributo alla collettività, "affogandolo" dentro qualche Geist (la cui universalità non è mai davvero tale, ma sempre connotata rispetto a una classe, una nazione, un momento storico, ecc.) che lo trascende. Vi sono però alcune specificità dell'idea di Peirce che la rendono interessante, e che pertengono al suo modo di fare filosofia.

La prima consiste nel fatto che "persona" è in Peirce, anzitutto, un carattere logico o semiotico (cfr. De Tienne 2005 , p. 91), sicché non ha senso, in questa prospettiva, distinguere 'persona' (questa-o-quella-persona) ed 'essere 
persona'. Detto altrimenti, con le parole dei logici, non si dà definizione estensionale della personalità: le persone non si contano (come possiamo contare - per esempio - i gorilla di montagna in libertà, per poi accorgerci che il loro numero diminuisce drasticamente, e che l'estinzione è un pericolo reale $)^{1}$, ma piuttosto possiamo dire che determinati aspetti della realtà (tra cui gli esseri umani) manifestano un carattere personale in quanto si prestano a essere "letti" formalmente, a essere interpretati, in un certo modo. Per questo userò spesso l'espressione 'persona(lità)'²: si tratta certamente di un gergo fastidioso per il lettore, ma mai quanto lo sarebbe la ripetizione continua di questa precisazione, che compio ora una volta per tutte.

La seconda pertiene alla forma sistematica della filosofia peirceana. Il fatto che Peirce abbia elaborato una forma di filosofia che è una semiotica ha spesso portato a "schiacciare" la sua riflessione sulla semiotica com'è oggi. Invece, per comprendere quest'autore senza incappare in qualche genere di riduzionismo, bisogna tenere presente in ogni momento il forte intento sistematico ${ }^{3}$ che lo anima. Inoltre, bisogna assumere la sostanziale unità della scienza (e di logica e filosofia entro l'impresa scientifica). È un'attitudine difficile da comprendere per la scienza specialistica che conosciamo, ma del tutto naturale, per così dire, per quella del xix secolo (in cui l'interdisciplinarità era regola, e non eccezione), di cui la semiotica è per certi versi l'ultima figlia (e lo mostra soprattutto nella sua vocazione all'enciclopedismo, che la scienza sociale specialistica lesse come imperialismo).

Insomma, la coerenza interna ed esterna costituisce un valore aggiunto in una filosofia fortemente sistematica come è quella di Peirce, tutta costruita sullo sviluppo combinatorio dell'impianto categoriale. È come dire, mutatis mutandis: ci sono singoli preludi e fughe del Clavicembalo

1. Cfr. De Tienne (2005, p. 101): «Solo un esame superficiale e disattento ci potrebbe portare a concepire la persona come un'entità singolare numerabile. Secoli di abiti, essenzialmente linguistici, ci hanno rafforzato in questa opinione» (lo stesso Peirce, qualche volta, soggiace a tali abiti: cfr. per esempio il testo in R 403 citato in quella stessa pagina).

2. Questa espressione si riferisce sostanzialmente a ciò che De Tienne (2005, pp. 102, 108) chiama "quasi-persona".

3. A partire dal riconoscimento di tale intento ho costruito la mia presentazione di Peirce (Fadda 2013), cui rimando il lettore meno familiare con l'autore. Altre introduzioni italiane sono Proni (1993) e Fabbrichesi (1993) e Maddalena (2015), che offre anche una sezione antologica. Sugli aspetti biografici cfr. Brent (1998). 
ben temperato che sono più orecchiabili, e meglio si prestano a essere ricordati - ma tutti ricevono un surplus di senso dal ricorrere riconoscibile del tema (nel caso delle fughe), e dal loro inserimento in un certo tipo di impianto generale (temperamento equabile, distinzione maggiore/minore). Così accade anche per l'idea (forse è un po' troppo forte parlare di 'teoria') peirceana della persona(lità).

Per presentarla, procederò così: dapprima mi riferirò alla sua "versione semiotica", precoce e luminosa (ma ancora allusiva e non sufficientemente astratta), rappresentata dalla dottrina dell'uomo-segno; poi individuerò una doppia radice metafisica dell'idea di persona, rappresentata dal monismo idealistico e dal sinechismo; a quel punto, sarò nelle condizioni di presentare la tesi adombrata nel mio titolo ('persona' è l'inverso di 'individuo'), mostrandone alcune conseguenze dal punto di vista morale; infine, affronterò il peculiare teismo di Peirce, che della sua idea di persona(lità) costituisce a mio avviso lo sviluppo più interessante, e uno dei meno enfatizzati da chi si accosta all'autore da un punto di vista semiotico. Non potrò sviluppare a fondo nessuno di questi temi, ma spero che l'abbozzare il percorso possa comunque aiutare a vedere la centralità della questione nell'ambito della filosofia di Peirce (e non solo), e l'interesse - non solo dal punto di vista storico-filosofico - di ulteriori ricerche ${ }^{4}$.

\section{La semiotica come porta d'accesso al tema della persona(lità)}

Are we shut up in a box of flesh and blood?

Charles S. Peirce (1867)

Come notato da De Tienne (2005, p. 98) e da vari altri, "una teoria della persona come effetto semiotico del lavoro

4. Il tema non è nuovo per gli specialisti di Peirce: ricognizioni generali - almeno a partire da Colapietro (1989) - sono state tentate più volte, e vari contributi importanti, in lingua italiana, si trovano in Calcaterra (2005). Tra questi, particolarmente rilevante è il già citato saggio di De Tienne (2005), che assumerò come principale termine di confronto nella prima parte di questo scritto. Quando si tratterà invece, nei paragrafi successivi, di esplorare le relazioni con la nozione di sentimento come base della filosofia pratica peirceana, e con la versione di teismo da Peirce propugnata, il principale riferimento per la discussione sarà Atkins (2016), dedicato a questi temi. Le implicazioni più vaste dell'idea peirceana di persona sono relativamente poco note (o quantomeno, meno considerate) dal pubblico più vasto di chi si occupa di semiotica e/o di filosofia; su alcune di esse ho provato a ragionare in (Fadda 2014a, 2014b, 2018). 

all'interno dell'esperienza" è rinvenibile già dalla prima fase della riflessione di Peirce. Rivolgiamoci dunque alle ultime pagine di Alcune conseguenze di quattro incapacità, tra le più citate del nostro autore. La trovata che apre la sezione finale del saggio ${ }^{5}$ è paradossale: Peirce immagina che le parole si rivoltino contro l'uomo (convinto, da parte sua, di poterne disporre a piacimento) per mostrargli che egli non è il loro padrone, ma che semmai - per molti versi - sono esse ad avere il dominio su di lui. Per questo le parole dicono all'uomo:

Tu non significhi niente che non ti abbiamo insegnato noi, e quindi significhi solo in quanto indirizzi qualche parola come l'interpretante del tuo pensiero. (CP 5.313 - 2003, p. 109)

Da qui, Peirce arriva alle sue conclusioni attraverso una serie di equazioni (un modulo di pensiero caratteristico del suo stile cognitivo $)^{6}$ : se l'uomo è pensiero, il pensiero è segno, il linguaggio è segno (o è fatto di segni - a partire dalle parole), allora l'uomo è un segno, proprio come lo è una parola.

D'altra parte, un segno da solo non è nulla. Il valore del segno sta nel farsi abito, perché solo una serie di segni coerenti è interpretabile. Ciò vale anche per l'uomo:

l'identità dell'uomo consiste nella coerenza fra ciò che egli fa e ciò che pensa, e la coerenza è il carattere intellegibile di una cosa: cioè il suo esprimere qualcosa. (CP 5.315 - ibid.)

La conclusione - dell'argomento e del saggio - è una svalutazione assoluta dell'individualità, compendiata da tre versi presi da Shakespeare ${ }^{7}$ : il nostro valore come persone non risiede in qualche presunta individualità inaccessibile,

5. CP 5.313-317 (= 2003, pp. 108 sgg.). Adopero l'abbreviazione consueta e il consueto sistema di citazione per volumi e paragrafi dei Collected Papers (Peirce 1931-58) per comodità, nonostante le pecche filologiche dell'antologia, che hanno portato alcuni specialisti a ritenerla inutilizzabile. Le traduzioni italiane, ove presenti, sono tratte da Peirce (2003).

6. In virtù del principio logico-semiotico espresso dalla massima nota notae est nota rei ipsius (Cfr. Bellucci 2017, pp. 42 e passim).

7. Shakespeare $(1603$, vv. $117,119-120)$. Il riferimento all'essenza vitrea ritornerà - non a caso - come titolo in uno dei saggi cosmologici che vedremo nel paragrafo seguente, nel cui contesto nasce l'idea peirceana di persona(lità). 
ma - al contrario - nella parte di noi che tutti vedono, e che tutti possono capire e imitare. Abbiamo possesso esclusivo solo degli scarti: solo i nostri difetti sono veramente nostri.

L'uomo individuale, dato che la sua esistenza separata si manifesta soltanto attraverso l'ignoranza e l'errore, nella misura in cui egli è qualcosa di scisso sia dai suoi compagni sia da ciò che essi sono destinati ad essere, è soltanto negazione. Questo è l'uomo, [...] uomo orgoglioso, / più ignorante di ciò di cui ha maggior certezza, / la sua vitrea essenza. (CP 5.317 - ibid.)

Le pagine peirceane sull'uomo-segno - al di là del loro valore per sé, e del fascino che hanno esercitato su generazioni di lettori - possono essere viste come una "porta" semiotica a un tema che è insieme più vasto e più astratto, e che, investigato fino in fondo, richiede a Peirce di trascendere la dimensione antropologica e psicologica che è, per certi versi, ancora dominante nei saggi "anticartesiani” di quegli anni. Per mostrarlo, dobbiamo entrare nella metafisica di Peirce, e fare un salto in avanti di 25-30 anni.

2. Due radici metafisiche della nozione peirceana di persona

Nor must any synechist say:

"I'm altogether me, and not at all you".

Charles S. Peirce (1892)

Diverse periodizzazioni sono state applicate alla lunga traiettoria intellettuale di Peirce. Ma la soglia di discontinuità più significativa si pone più o meno a due terzi della sua vita, intorno ai 50 anni, quando abbandona (per cause di forza maggiore) il suo lavoro di scienziato, e si ritira in una casa colonica di Milford, in Pennsylvania, a scrivere. Pur pressato da necessità economiche e costretto a fare una quantità enorme di lavoro intellettuale "di servizio" (recensioni, voci di enciclopedia, e così via), si ritrova in qualche modo padrone di se stesso. L'esigenza di una filosofia sistematica diviene allora più pressante - o (il che cambia poco le cose) diviene maggiore il tempo per perseguirla. Questa sorta di ritiro in sé medesimo, forzato dagli eventi e assecondato dal pensiero, produce anzitutto una maggiore attenzione al tema della metafisica: gli anni Novanta possono essere considerati come il periodo in cui esso riveste un'importanza 
maggiore per il padre della semiotica. All'inizio e alla fine di questa decade Peirce produce i frutti più interessanti per noi: rispettivamente, la cosmologia dei saggi su The Monist (pubblicati tra il 1891 e il 1893), e poi la dottrina della continuità nella versione presentata nelle Cambridge Conferences del febbraio-marzo $1898^{8}$.

La "serie cosmologica" del The Monist 9 segna un cambiamento importante nella filosofia di Peirce (e si correla a cambiamenti importanti della sua vita, come abbiamo accennato). In essa appare la nozione matura di 'persona' che è oggetto di questo lavoro, nel contesto di una metafisica idealista (influenzata dalla disputa medievale sugli universali, ma anche da ricerche biologiche). In questo contesto, però, "smonterò" i due elementi, definendo dapprima la cornice idealistico-cosmologica, per lasciare la presentazione della nozione di persona(lità) alla sezione seguente.

La cosmologia prende avvio dall'affermazione recisa di un monismo idealistico (cfr. CP 6.24 - 2003, pp. 343 sg.). Peirce lo enuncia in modo un po' secco, ma trasparente: dapprima rifiuta il dualismo cartesiano tra mente e materia, e l'indipendenza tra leggi fisiche e leggi psichiche, in base al rasoio di Ockham. Posto allora a scegliere se le leggi psichiche dipendano da quelle fisiche o viceversa, opta per la seconda opzione, in base all'idea che le leggi fisiche non siano suscettibili di sviluppo, mentre quelle mentali sì (del resto, se il pensiero è segno, e il segno è sviluppo, non potrebbe essere altrimenti). Lo sviluppo della mente può talvolta arrivare a un binario morto, in cui si arresta e diviene assolutamente prevedibile; una legge fisica, secondo Peirce, non è altro che questo: un abito che non può cambiare, una possibilità di interpretazione chiusa a ogni sviluppo. Egli compendia questa idea attraverso il ricorso

8. È uso individuare diverse fasi del pensiero di Peirce a partire da serie di articoli (per es.i cosiddetti "saggi anticartesiani" del 1868-9, cui si è accennato sopra), o conferenze (per es.le Harvard Lectures della primavera del 1903). Questo modo di procedere nella ricostruzione storica non è dettato solo da esigenze di comodità, ma corrisponde in effetti a un modo di lavorare dello stesso autore (in qualche modo, sono libri che non vogliono, o non sanno, diventare tali). Sulle diverse versioni della dottrina della continuità cfr. sotto n. 13.

9. The Architecture of Theories (gennaio 1891); The Doctrine of Necessity Examined (aprile 1892); The Law of Mind (luglio 1892); Man's Glassy Essence (ottobre 1892); Evolutionary Love (gennaio 1893); Reply to the Necessitarians (luglio 1893). Traduzioni italiane in Peirce (2003, pp. 333-350, 1071-1168). 
10. Effetus è utilizzato in latino per designare il bestiame che ha appena figliato, ma anche, per traslato, i campi lasciati a maggese, e anche un corpo vecchio e incapace di azione. L'etica della terminologia di Peirce (cfr. CP 2.219-26 - 2003, p. 1284), che lo spingeva a creare termini brutti, ma efficaci e impermeabili a un'estensione semantica "selvaggia" (cfr. CP 5.414), e talvolta perfino a intervenire sui sistemi di suffissazione, non andava disgiunta da una certa raffinatezza lessicale.

11. Sulle implicazioni diffuse del sinechismo nella filosofia di Peirce cfr. Parker (1998).

12. Pietarinen (2010) mostra come il pensiero di Peirce mostri analogie locali, ma anche incompatibilità, con varie posizioni espresse nel xx secolo, ma si sottrae a una comparazione complessiva per le sue peculiarità, a partire dalla scelta delle nozioni di base (per es.'collezione' o 'moltitudine'). Per una presentazione agile e rigorosa della filosofia della matematica del Novecento cfr. Cellucci (2007). 
adottarne. Per comprendere appieno il valore della continuità, dobbiamo ricordare che la terzità è generalità, e la continuità è la forma più generale di terzità. Ma come la generalità può essere resa più o meno generale, la continuità può essere resa più o meno continua. L'esempio portato da Peirce nell'ultima delle Cambridge Conferences del 1898 (cfr. CP 6.185 sgg. - 2003, pp. 1173 sgg.), sebbene non rappresenti l'ultima parola di Peirce in materia ${ }^{13}$, può essere utile in ragione della sua semplicità. Peirce parte dall'atto più semplice: tracciare una linea di gesso sulla lavagna. La linea e la lavagna sono due continui: la linea è un continuo a una dimensione, la lavagna un continuo a due dimensioni. La linea trae la sua continuità dalla lavagna, e rappresenta una discontinuità rispetto a essa, dividendo la lavagna in ciò che è linea e ciò che è non-linea. Ma "dentro" la linea non vi è differenza o discontinuità ${ }^{14}$.

Quest'idea fondamentale per cui il discreto si ottiene dal continuo, e non viceversa (cfr. Havenel 2015, p. 115) va in direzione inversa a quella oggi più accettata, detta di Cantor-Dedekind, che parte invece dai punti. Per questo Moore (2015) chiama questo approccio, definibile attraverso la proprietà della riflessività spiegata da Zalamea (2012) - per cui ogni parte riflette l'intero -, 'pre-puntuale': infatti i punti non possono entrare in un tale continuo, giacché non sono parte se non di se stessi. Se partiamo dunque da un continuo con dimensione $n$ e un altro con dimensione $n-1$, possiamo immaginare una fuga in avanti (continui fatti di continui) e una fuga all'indietro (continui “un po' meno

13. Sull'evoluzione delle ricerche di Peirce sulla nozione matematica di continuo cfr. Havenel $(2008,2015)$. Le cinque fasi individuate da questo autore sono: antinominalistica (fino al 1884), cantoriana (fino al 1892), infinitesimale (fino al 1897); supermultitudinaria (fino al 1907), e infine topologica. L'esempio appartiene dunque all'esordio della quarta fase, che, con l'introduzione di un continuum dimensionale (si veda infra) è quella fondamentale per la cosmologia evolutiva (cfr. Havenel 2015, p. 119). Sebbene la concezione topologica sia ancora più radicale (e più funzionale dal punto di vista filosofico), credo anch'io, come Moore (2015, p. 128 n.1) che la concezione "matura" nel suo complesso - quella in cui Peirce prende la via opposta rispetto a Cantor - sia innescata proprio da La legge della mente (e dunque da fattori non riducibili a quelli meramente matematici).

14. L'esempio anticipa la costruzione dei grafi esistenziali, in cui il continuo di ordine superiore (foglio di asserzione) è lo sfondo su cui si staglia l'identità, rappresentata da una linea (ma quella linea è a sua volta un continuo). 
continui" di quelli che discretizzano, ma pur sempre continui) senza mai un approdo in un senso e nell'altro.

Continuità e agapasmo rappresentano insomma le due facce di una stessa medaglia, e permettono di dare corpo alla nozione di persona proprio - se mi si passa il gioco di parole - togliendole corpo. Vediamo perché.

\section{La persona come sviluppo non-individuale}

there is nothing which distinguishes my personal identity except my faults and my limitations.

Charles S. Peirce (1898)

Il concetto maturo di persona(lità) risulta dall'acquisizione della prospettiva cosmologica e sinechistica. Ma se vogliamo tornare per un attimo alla prospettiva semiotica (che non per questo risulta "tradita" o messa da parte, come abbiamo visto da De Tienne 2005), potremmo dire che il soggetto è mente in quanto interprete di segni, e persona in quanto segno egli stesso. In ogni caso, la base di tutto è l'opposizione tra persona e individuo, e l'idea che la definizione principale di ogni carattere personale sta nel suo essere potenzialmente condiviso, e non irriducibilmente individuale.

Iniziamo dalla pars destruens. Abbiamo già visto le formulazioni di Peirce risalenti al 1868 in cui l'individualità era presentata sostanzialmente come un disvalore. Quelle forse più simili e più radicali nel presentare "una concezione non fondativa, ma privativa del self" (Colapietro 2005, p. 48) risalgono a un testo degli anni 1898, Filosofia e condotta di vita, che inaugura le Cambridge Conferences, nelle quali si presenta la dottrina della continuità che ho appena (brevemente) esposto. Si tratta di una lezione particolare, che per Atkins (2016, p. 6) rappresenta in assoluto il testo più controverso e dibattuto di Peirce. Sappiamo che egli aggiunse questa prima conferenza sotto insistenza di William James, che temeva che il ciclo di lezioni incentrato sul tema della continuità risultasse troppo astratto per il pubblico previsto, e chiese all'amico di fare riferimento a vitally important topics (cfr. Brent 1998, pp. 262 sgg.). Latteggiamento paradossale che domina questa conferenza rappresenta inoltre una risposta diretta a The Will to Believe dello stesso James (cfr. 
Atkins 2016, cap. 1), uscita da poco, in cui però si trovavano varie affermazioni che Peirce non poteva avallare ${ }^{15}$.

Partendo da una critica dell'intellettualismo etico introdotto nella filosofia attica al tempo di Socrate, e contrapponendola alla tradizione ionica di una filosofia scientifica (che da Talete va fino ad Aristotele) ben attenta a preservare la separazione tra filosofia scientifica e condotta di vita ${ }^{16}$, Peirce arriva dunque a una discussione sul tema della condotta, in cui si pongono in primo piano alcuni aspetti relati alla nozione di persona, e in particolare la nozione di 'sentimento' (sentiment - da non confondersi con feeling!), che è forse la principale della sua filosofia pratica.

Il nostro più profondo sentimento emette il verdetto della nostra insignificanza. L'analisi psicologica mostra che non vi è nulla che distingua la mia identità personale eccetto i miei errori e le mie limitazioni - oppure, se volete, il mio cieco volere, che è mio supremo sforzo annullare. (CP $1.673-2003$, p. 1201)

I sentimenti sono definiti da Savan (1981, p. 331) come "enduring system of emotions", e potremmo dunque assimilarli ad abiti di primità ${ }^{17}$. Il portato immediato, antropologico, del primato del sentiment è l'affermazione di una morale anti-eroica: se la condotta è dettata dal sentimento (e come tale assimilabile agli istinti animali), rinunciare all'egoismo non è una cosa particolarmente notevole. Ogni uomo che

15. Questo non significa, naturalmente, che non vi siano, nell'ambito della filosofia pratica, alcune premesse condivise tra Peirce e James (e nell'ambito della tradizione, pragmatista in generale) - prima tra tutte la svalutazione del primato della coscienza a favore di quello dell'esperienza: cfr. Fabbrichesi (2014).

16. Meno corretto mi pare opporre tradizione "greca" e "macedone", come fa Atkins (2016, p. 23).

17. Cfr. anche Hookway (1997, p. 216 «...a stable system of emotional attitudes»), e Acosta (2019), che riconosce anche la possibilità di educare i sentimenti (come avviene, appunto, con gli abiti) in ragione della loro natura di sistemi logici di emozioni. Non si tratta però di una posizione universalmente condivisa tra i commentatori. In particolare, Atkins (2016, p. 54) afferma che «characterizing sentiments as habits is not quite accurate», perché «[f]or Peirce, sentiments are feelings and feelings are Firsts». Per l'autore, i sentimenti sarebbero dunque meri feelings, primità semplici, colte semplicemente nel loro essere innescate da abiti (istinti o abituazioni molto precoci) e nella loro funzione locale di causazione del comportamento («conscious effectuation»-ivi, p. 55). Questa definizione mi sembra mal conciliabile con quella di Savan (pure accolta dall'autore), nonché con il fatto che lo stesso Atkins in altre parti del testo (cfr. per esempio pp. 66, 75) caratterizza i sentiments come generalizzazioni. 
sia uomo, componente a pieno titolo della propria specie, deve essere morale, e porre al primo posto il bene di tutti:

Negli animali in cui gli istinti sono più visibili, essi presentano la caratteristica di essere diretti principalmente, se non del tutto, alla preservazione della specie e di beneficiare pochissimo l'individuo. [...] $\mathrm{E}$ un dato di fatto che tale istinto ci stimola, in tutte le crisi vitali, a considerare le nostre vite individuali come piccole faccende. Fare questo non è uno straordinario vertice di virtù: è la caratteristica di ogni uomo o donna che non sia disprezzabile. (CP 1.639 - 2003, p. $1191 \mathrm{~s}$.

Il legame tra i temi di questa prima conferenza e quelli delle seguenti è meno estrinseco di quanto si possa pensare a prima vista. La svalutazione dell'individualità, se dal punto di vista teorico è una conseguenza della dottrina della continuità, da un altro punto di vista ha un valore propedeutico per l'uditorio di Peirce: seppure non sia possibile a chi ascolta entrare nei dettagli tecnico-matematici della continuità, il sentimento della pochezza del nostro io individuale è una porta che non sostituisce un approccio scientifico (e non va confuso con esso), ma aiuta a trovare una forma di comprensione.

Veniamo ora invece alla definizione di persona(lità), che (come quella di segno, e tante altre in Peirce) è astratta e potente. Come l'idea matura di continuità, essa è legata alla legge della mente, e anzi ne è una conseguenza diretta - infatti la ritroviamo proprio nel saggio in cui la legge è enunciata (e che risale, ricordiamolo, al 1892).

La personalità è una forma di coordinazione o connessione di idee. [...] una connessione di idee è essa stessa un'idea generale e un'idea generale è un feeling vivente. [...] Questa personalità, come ogni idea generale, non è una cosa che si può apprendere in un istante. Deve essere vissuta nel tempo, e nessun tempo finito può contenerla in tutta la sua pienezza. $[\ldots]$

La parola coordinazione, pero, implica un po' più [dell'autocoscienza immediata]: implica un'armonia teleologica delle idee. E nel caso della personalità questa teleologia è più che il perseguimento volontario di un fine predeterminato: è una teleologia in evoluzione. Questo è il carattere personale. Un'idea generale, vivente e cosciente in questo istante, determina già atti nel futuro in una misura di cui 
ora non è cosciente. Questo riferimento al futuro è un elemento essenziale della personalità.

Se i fini di una persona fossero già esplicitati non resterebbe spazio per lo sviluppo, la crescita, la vita e, di conseguenza, non ci sarebbe personalità. Il semplice portare a termine propositi predeterminati è meccanico. (CP 6.1557 - 2003, p. 1119)

La persona(lità) è dunque - in accordo con la legge della mente - mente che si sviluppa, che esibisce insieme coerenza e innovazione. Questo carattere, però, non è legato a nessuna cosa in particolare. Ci possono essere, ad esempio, persone sovraindividuali:

Ci deve essere qualcosa come una coscienza personale nei gruppi di uomini che sono in comunione intima e intensamente simpatetica. [...] L'esprit de corps, il sentimento nazionale, la simpatia, non sono pure metafore. Nessuno di noi può pienamente comprendere che cosa siano le menti delle corporazioni, non più di quanto una delle mie cellule cerebrali possa sapere ciò che l'intero cervello stia pensando." (CP 6.271 - 2003, p. 1141 s.)

E inversamente, niente impedisce che due (o più) personalità si annidino nel medesimo corpo:

Lidea di una seconda personalità, il che equivale a dire la seconda personalità stessa, entra nel campo della coscienza diretta della prima persona, ed è immediatamente percepita come il suo ego, benché con minor forza." (CP $6.160-2003$, p. 1120)

L'unico aspetto fondamentale è che la coerenza esibita dalle persona(lità) non sia meccanica e predeterminata: un fiume che scorre e si scava il proprio letto esibisce un abito mentale (cfr. CP 5.492) ma non un carattere personale. La necessità - il grande nemico di Peirce nei saggi cosmologici - equivale al perseguimento di fini immutabili, e stabiliti una volta per tutte. La coerenza genuina che definisce la persona(lità) implica invece che si possa (e a volte si debba) cambiare idea. Questa ci sembra una caratteristica sensata quando parliamo di uomini, mentre ci appare straniante se parliamo di Dio (che pure è - o dovrebbe essere - una persona). Eppure, per Peirce, è proprio così. 
4. Sul carattere personale di Dio

The word 'God' [...]

is the definable proper name.

Charles S. Peirce (1908)

Il teismo di Peirce non andrebbe considerato come una debolezza personale, o come una semplice concessione all'ambiente in cui egli si era formato: al contrario, si tratta di un aspetto del suo pensiero che non solo è coerente con gli altri, ma che compendia per certi versi il suo sistema filosofico. Non è questo il luogo per mostrarlo in esteso ${ }^{18}$, ma mi limiterò a mettere brevemente in rilievo gli aspetti connessi alla nozione di persona, e che mi portano a radicalizzare l'idea dell'opposizione tra persona e individuo.

Come è noto, la "prova" peirceana della realtà di Dio (non della sua esistenza, come vedremo fra poco) è abbastanza particolare, e più simile a una ricetta di cucina che a un teorema di geometria ${ }^{19}$. Si tratta di un invito alla pratica quotidiana del musement, il libero gioco dell'immaginazione che si oppone al pensiero scientifico: laddove, infatti, quest'ultimo è vincolato alla catena abduzione-deduzioneinduzione (faccio un'ipotesi, ne traggo tutte le conseguenze, verifico se queste conseguenze si danno), il musement consiste, all'inverso, nello scacciare qualsiasi ipotesi a mano a mano ch'essa si presenti, e nel lasciar correre l'immaginazione senza regole. Se, praticando correttamente e costantemente questo gioco, il pensiero di una divinità si presenterà invariabilmente, allora ciò significherà che si tratta di un ente necessario (come oggetto di pensiero) ${ }^{20}$. Se Laplace,

18. Saggi pionieristici in questo campo sono Orange (1984) e Raposa (1989). Discussioni di dettaglio sugli argomenti di Peirce in favore del teismo si trovano in Atkins (2016; cfr. infra). Sul legame tra la dimensione teologica e quella estetica nel pensiero di Peirce cfr. Barrena (2017).

19. A Neglected Argument for the Reality of God, 1908 (CP 6.452-85 - 2003, pp. 1233-1254). L'analisi di questo saggio condotta da Atkins (2016, pp. $85 \mathrm{sgg}$.) parte da due presupposti a mio avviso non sufficientemente argomentati: 1) il testo ha uno scopo più modesto di quello dichiarato: si tratta solo di difendere la plausibilità di un'ipotesi divina ottenuta con corsi di pensiero non formali/controllati; 2) l'idea che la divinità non può essere un esistente (per cui cfr. sotto) non è pertinente al ragionamento sviluppato da Peirce (ivi, p. 114). Sulla prima ho motivi di dissentire (si veda la nota seguente); quanto alla seconda, sono interessato alle conseguenze di questa idea non nel contesto di quello scritto, ma in relazione alla definizione di una nozione di persona (che in quel testo non è posta a tema).

20. Di fatto, nonostante l'origine religiosa, il tema del musement è stato sviluppato in 
rispondendo a Napoleone, aveva detto di non aver bisogno di Dio come ipotesi, Peirce risponde(rebbe) che la realtà di Dio risiede esattamente nel non essere strumentale, nell'affacciarsi inevitabilmente al pensiero nonostante non serva a niente (o a niente di particolare, per essere più precisi). Un modo in cui egli esprime questo carattere è dicendo che si tratta dell'unico nome proprio ${ }^{21}$ necessario: un uno che è il tutto (e non possono esservene altri).

La realtà di Dio è dunque tutta mentale: è terzità di terzità, (matematicamente, il continuo dei continui: cfr. Havenel 2015, pp. 109 sg.). Ma se l'esistenza è secondità, e la divinità è terzità completamente dispiegata, ciò implica che Dio non deve esistere. L'idea che l'esistenza sia una perfezione, cardine delle prove dell'esistenza divina, da Anselmo d'Aosta a Gödel, è rifiutata da Peirce: «Sarebbe feticismo dire che Dio esiste» (CP 6.495). Tutto ciò che esiste influisce, al modo della secondità, su altri esistenti, ma non è suscettibile di generalizzazione infinita: per questo, nel sistema peirceano, un Dio esistente sarebbe imperfetto (sarebbe una cosa).

Il carattere evolutivo della metafisica e della cosmologia di Peirce impone però che questa terzità dispiegata, continuo dei continui, uno-tutto, non sia sempre uguale a sé stessa. Il Dio peirceano non è un uno parmenideo, ma cambia. È teleologia in evoluzione, e insieme limite pensabile di questa teleologia. La metafora matematica con cui questo viene rappresentato è quella dell'iperbole,

contesti diversi, e innanzitutto nei testi dedicati alla semiotica della detection, da Eco \& Sebeok (1983) in poi. Atkins (2016, pp. 88 sgg.) tende a considerare il ricorso al musement puramente esemplificativo: esso sarebbe per lui solo uno dei corsi di pensiero che possono portare ad apprezzare la plausibilità di un'ipotesi divina. Questo mi appare come una forzatura del dettato di Peirce (CP. 6.462), che si riferisce sì a «wonderfully different ways of thinking», ma sempre all'interno del musement. Per conto mio, è invece proprio la natura specifica del musement (la determinazione nel "lasciar correre" il pensiero senza entrare nel circuito abduzione-deduzione-induzione tipico dell'inquiry) ciò che dovrebbe far propendere per l'idea che si tratta davvero di un argomento per la "realtà" di Dio: un ente non esistente, che si presenta però come ipotesi «irresistible» (CP 6.480), per quanto non provata e non provabile scientificamente, e che raccomanda un certo tipo di ideale e di condotta.

21. CP 6.542. Nel considerare questa definizione, dobbiamo tenere a mente che il nome proprio non è (se non secondariamente) il nome che pertiene a una cosa, e solo a quella, ma nella tradizione grammaticale e logica è anzitutto "ciò che propriamente è nome" (onoma kyrion). Dire che 'Dio' è l'unico nome proprio definibile significa, dunque, che la sua unicità non è contingente (come nel caso del sole, che è un nome comune ma diventa $d i$ fatto il nome d'unica entità) ma necessaria: c'è necessariamente una divinità, e solo una, e questa divinità deve avere un nome in senso proprio. 
curva in sviluppo continuo che ha un inizio e una fine solo ideali (cfr. CP. 6.581). L'alfa e l'omega dell'Apocalis$s e$, dunque, possono essere visti come simbolo di questo inizio e questa fine ideali, presupposti necessari ma non attingibili ${ }^{22}$, il primo e il secondo assoluti da cui la terzità dispiegata prende origine (cfr. Orange 1984, pp. 42 sgg., Raposa 1989, pp. 79 sg.).

Quelle che ho appena esposto, d'altra parte, non sono altro che le caratteristiche della nozione di persona, semplicemente generalizzate e portate al loro limite. Come Dio è il limite del mondo, dunque, la persona-Dio è il limite della nozione di persona, pensabile (e necessario), ma non attingibile dalle persone-che-siamo. Dire che una persona è un'immagine di Dio, allora, significa dire che esibisce una teleologia in evoluzione, e una possibilità indefinita di generalizzazione (giacché essere perfettibile è la sola forma di perfezione), che tende a svincolarsi da ogni necessità e dal legame con la secondità. Significa, per chi possiede questo carattere, essere più che sé stesso - per quanto l'uomo lo possa essere - e aprirsi a tutto ciò che è sviluppo, a volte inaspettato ma ragionevole, nel mondo ${ }^{23}$. Non è poco.

\section{Conclusioni}

Le pagine sull'uomo-segno - a rileggerle nel contesto generale - presentano già in nuce (o in atto) i principali caratteri della teoria peirceana della persona(lità): ciò che conta è un'associazione coerente di idee, suscettibile di essere interpretata e integrata con altre associazioni coerenti di idee, e per questo l'individualità è da considerarsi un male, la radice di ciò che osta alla propagazione progressiva delle idee buone. La forza di quella metafora sta anzitutto nel valore di presa di coscienza, di appello immediato inserito in una dimensione antropologica - e infatti le pagine sulla persona(lità) degli anni Novanta, non meno paradossali, non sortiscono quello stesso effetto.

22. Se torniamo alla metafora della continuità vista sopra, essi corrisponderebbero alla discontinuità assoluta e alla continuità non ulteriormente generalizzabile.

23. Cfr. Havenel (2015, p. 109): «We can now see the connection Peirce found between his conception of continuity and spirituality; with the idea that there is between each one of us and the Creator, a real continuity» La "ragionevolezza ragionevole" (reasonable reasonableness) è individuata da Peirce (cfr. CP 5.121) come oggetto proprio della metafisica, e in qualche modo è una definizione di Dio, inteso come terzità assoluta. 
Ma se ci limitassimo a trasporre in teoria quanto si dice lì, ne risulterebbe una posizione simile a quella della memetica nel senso di Dawkins ${ }^{24}$ : come i geni si servono degli organismi per proliferare, i memi si servono delle menti singole (o meglio, dei cervelli degli individui) per diffondersi ${ }^{25}$. Ma nella prospettiva dawkinsiana vi sono due aspetti che a Peirce sarebbero piaciuti poco: il primo è l'interpretazione ristretta dello struggle for life darwiniano - contro cui egli si scaglia più volte, specialmente quando prende la forma del cosiddetto "darwinismo sociale" 26 - e il secondo è l'idea che gli organismi siano semplici agenti inconsapevoli di questa diffusione e di questa lotta.

La concezione matura della persona(lità), e il suo esito teistico, non assolvono dunque soltanto a un compito di architettonica intellettuale, ma rivestono un'immensa portata morale e interpellano il singolo in maniera non immediata, ma potente: se combattere contro ciò che vi è in me di individuale, e collaborare allo sviluppo coerente del pensiero, è scelta consapevole e autocontrollata, allora ciò equivale a farsi collaboratore (o complice) della creazione (cfr. CP 6.289; Orange 1984, p. 45; Barrena 2015, pp. 148 sgg.) - che è per Peirce, come abbiamo visto, uno sviluppo progressivo senza inizio né fine. Lungi dall'essere annullati, il valore e la responsabilità del singolo ne risultano rinforzati al massimo grado.

\section{Bibliografia}

Acosta Lopez de Mesa J.

2019 A semiotic theory of self-control, «Cognitio», Febr. 21, pp. 217-229.

24. Cfr. Dawkins (1976, cap. 11). L'accezione oggi più diffusa di 'meme', com'è noto, deriva in larga misura da questa, ma ha assunto un significato più ristretto (così come, p. es., 'annegare' passò a significare "uccidere per acqua" e poi "morire per acqua" dal significato più generale di "dare la morte": cfr. Saussure 1922, p. 93, da cui traggo l'esempio, con modifiche).

25. Credo che De Tienne (2005, p. 99) abbia in mente anche un pericolo di questo genere, quando precisa che la concezione peirceana «non [equivale a] dire che Peirce riduca l'ego a un mero luogo dove si manifesta un continuo simbolico. Una persona non può ridursi a un luogo di espressione semiotica. Se un simile luogo esiste, si tratta di un organismo vivente».

26. Cfr. CP 6.294, in cui Peirce oppone il «Gospel of greed» del darwinismo sociale al vangelo cristiano. 
Atkins R. K.

2016 Peirce and the Conduct of Life. Sentiment and Instinct in Ethics and Religion, Cambridge University Press, Cambridge.

\section{Barrena S.}

2015 La belleza en Charles S. Peirce: Origen y alcance de sus ideas estéticas, EUNSA, Navarra.

Bellucci F.

2017 Peirce's speculative grammar. Logic as semiotics, New York, Routledge, New York.

Brent J.

1998 Charles Sanders Peirce: A Life, Indiana University Press, Bloomington-Indianapolis, $2^{\text {nd }} \mathrm{ed}$.

Calcaterra R.M. (a cura di)

2005 Semiotica e fenomenologia del sé, Nino Aragno, Torino.

Cellucci C.

2007 La filosofia della matematica del Novecento, Laterza, Roma-Bari.

Colapietro V.M.

1989 Peirce's Approach to the Self, SUNY Press, Albany (NY).

2005 L'altro come sé e il sé come altro, in Calcaterra 2005,

Dawkins R. pp. 45-68.

1976 The Selfish Gene, Oxford University Press, Oxford (trad. it. Il gene egoista, Mondadori, Milano 1994).

De Tienne A.

2005 La persona come segno, in Calcaterra 2005, pp. 91-110.

Eco U., Sebeok Th.A. (a cura di)

1983 Il segno dei tre: Holmes, Dupin, Peirce, Bompiani, Milano. Fabbrichesi R.

1993 Introduzione a Peirce, Laterza, Roma-Bari.

2005 Esiste la coscienza? Le tesi inattuali di James a confronto con la filosofia novecentesca, «RIFL», SFL 2014, pp. 152163.

Fadda E.

2013 Peirce, Carocci, Roma.

2014a Esprit de corps. Sulla rilevanza della nozione peirceana di 'persona' per lo studio della nozione di identità nei social media, «Ocula», XV, pp. 1-14.

2014b Persone reali. Alcune note sulla rilevanza della nozione peirceana di 'realtà' per l'ontologia sociale, in P. Garofalo (a cura di), Frammenti di realtà sociale, Limina Mentis, Villasanta, pp. 61-80. 
2018 Troppo lontani, troppo vicini. Elementi di prossemica virtuale, Quodlibet, Macerata.

Gödel K.

2006 La prova matematica dell'esistenza di Dio, con scritti di G. Lolli e P. Odifreddi, Bollati Boringhieri, Torino.

Havenel J.

2008 Peirce's Clarifications of Continuity, «Transactions of the Charles S. Peirce Society», LXIV/1, 2008, pp. 86-133.

2015 Peirce's Meditations on Continuity: from Transitivity to Topology, «Cuadernos de sistemática peirceana», 7, pp. 101126.

Hookway Ch.

1997 Sentiment and Self-Control, in J. Brunning, P. Forster (a cura di), The Rule of Reason: The Philosophy of Charles Sanders Peirce, University of Toronto Press, Toronto, pp. 201-222.

James W.

1896 The Will to Believe, «The New World», 5, pp. 327-347. Kafka F.

1946 Betrachtungen über Sünde, Hoffnung, Leid und den wahren Weg, in Gesammelte Werke (tr. it. Aforismi di Zûrau, a cura di R. Calasso, Adelphi, Milano, 2004).

Maddalena G.

2015 Peirce, La Scuola, Brescia.

Moore G.E. (a cura di)

2010 New Essays in Peirce's Mathematical Philosophy, Open Court, New York.

2015 Peirce's Prepunctual Continuum, «Cuadernos de sistemática peirceana» 7, pp. 127-138.

Orange D.

1984 Peirce's conception of God. A developmental study, Institute for Studies in Pragmaticism, Lubbock.

Parker K.

1998 The Continuity of Peirce's Thought, Vanderbilt University Press, Nashville-London.

Peirce Ch.S.

1931-58 Collected Papers [= CP], a cura di Ch. Hartshorne, P. Weiss, A. W. Burks, Harvard University Press, Cambridge (Mass.), 8 voll. (trad. it. parziale Opere, a cura di M.A. Bonfantini, Bompiani, Milano 2003).

1967 Annotated Catalogue of the Papers of Charles S. Peirce $[=\mathrm{R}]$, a cura di R.S. Robin), University of Massachusetts Press, Amherst 1967.

2003 Opere, a cura di M. Bonfantini, Bompiani, Milano. 
2010 Philosophy of Mathematics, a cura di G.E. Moore, Indiana University Press, Bloomington.

Pietarinen A.-V.

2010 Which Philosophy of Mathematics is Pragmaticism?, in Moore 2010, pp. 41-61.

Proni G.

1990 Introduzione a Peirce, Bompiani, Milano.

Raposa M.L.

1989 Peirce's Philosophy of Religion, Indiana University Press, Bloomington.

Saussure F. de

1922 Cours de linguistique générale, a cura di Ch. Bally, A. Sechehaye, Payot, Genève-Paris (trad. it. Corso di linguistica generale, introduzione, commento e note a cura di T. De Mauro, Laterza, Roma-Bari 1967 sgg.).

Savan D.

1981 Peirce's Semiotic Theory of Emotion, in K.L. Ketner et al. (a cura di), Proceedings of the C. S. Peirce Bicentennial International Congress, Texas Tech University Press, Lubbock, pp. 319-334.

Shakespeare W.

1603 Measure for measure (trad. it. Misura per misura, Feltrinelli, Milano 2003).

Zalamea F.

2012 Peirce's Logic of Continuity: A Conceptual and Mathematical Approach, Docent Press. 


\section{L'uattitudine persona» o della capacità di trasformare: una prospettiva pedagogica}

Sara Nosari

La persona è e deve essere riconosciuta come "questione". Infatti, se da un lato permette di configurare l'essere umano e la sua esistenza all'interno di una narrazione di senso in grado di attribuire un posto, un ordine e una direzione a ogni elemento coinvolto, dall'altro espone lo stesso essere umano e la stessa esistenza a una continua problematizzazione che ne scopre implicazioni, conseguenze e sfide ${ }^{1}$. Lo sguardo pedagogico partecipa a questa problematizzazione con una particolare "soluzione" che propone implicazioni, conseguenze e sfide che comprendono la persona "legandola"2 all'idea di cambiamento.

Lo sguardo pedagogico, infatti, legge e interpreta la realtà, alla luce della processualità che caratterizza qualsia-

1. La persona è "questione" fondamentalmente per il fatto che la sua "idea-realtà" - come la definisce Vittorio Possenti (2013) - "scuote" l'esperienza che l'uomo fa della propria esistenza, richiamando l'attenzione sulla differenza umana e sulla sua eccezionalità.

2. Il legame in questione non ha unicamente la funzione di rimandare la persona all'idea di cambiamento. Il legame con cui lo sguardo pedagogico rilegge la scena dell'esistenza umana segna una particolare compromissione: la persona è da riconoscere come possibile principio di cambiamento. In quanto tale, è "promessa" di cambiamento e nel cambiamento ha una "missione" (Bellingreri 2005). 
L'«attitudine persona» o della capacità di trasformare si esperienza. Tuttavia, se è vero che non può prescindere dall'idea stessa di cambiamento, è altrettanto vero che tale sguardo si occupa di cambiamento non solo dal punto di vista della programmazione, della verifica e della misurazione. Lo sguardo pedagogico si (pre)occupa di cambiamento soprattutto perché è impegnato a progettarlo: il suo "taglio" infatti com-prende l'esperienza della realtà per aprirla a possibilità ulteriori, creando "vincoli", segnando "distanze" o costruendo "ponti" all'interno della concatenazione delle “cose come sono" (Bateson 1997, p. 398). L'ulteriorità che la lettura pedagogica prospetta non è ovviamente numerica: non si tratta di una possibilità logica, ma di una possibilità di potenza. È dunque uno sguardo che pone le premesse per un'azione di cambiamento che si propone quale reale e concreta trasformazione.

Il carattere trasformativo della proposta pedagogica, tuttavia, non è da pensare come esauribile nel passaggio predeterminato da una fase iniziale a una fase finale (non ha valenza pedagogica la trasformazione del bruco in farfalla!). La sua azione è volta non solo a esplicitare, a potenziare o a mettere in evidenza capacità che ci sono già, ma anche a sviluppare capacità che sono ancora latenti o solo abbozzate $^{3}$. Linteresse pedagogico non è quindi rivolto a quelle possibilità di cambiamento in qualche modo anticipate da cause antecedenti o da concause presenti. Lo sguardo pedagogico ricerca possibilità autenticamente trasformative,

3. Si tratta di quel "rivoluzionario" modo di cambiare che è in grado di creare piani ulteriori. Paulo Freire ne evidenzia la valenza pedagogica: «Mi piace essere uomo, essere persona, perché so che il mio passaggio attraverso il mondo non è già stato determinato, non è prestabilito. Perché so che il mio "destino" non è un dato, ma qualcosa che deve essere ancora realizzato e dalla cui responsabilità non posso esimermi. Mi piace essere persona perché la Storia, in cui mi realizzo con gli altri e della cui costruzione faccio parte, è un tempo di possibilità e non di determinismo. Da qui deriva la mia grande insistenza sulla problematizzazione del futuro e il mio convinto rifiuto della sua inesorabilità. Mi piace essere persona perché in fondo percepisco che ha molto a che vedere con me stesso la costruzione della mia presenza al mondo, una presenza impossibile da comprendere al di fuori della tensione tra ciò che eredito geneticamente e ciò che eredito socialmente, culturalmente e storicamente. Non posso percepirmi come presenza nel mondo e, allo stesso tempo, spiegarla come il risultato di operazioni a me assolutamente estranee. La mia presenza nel mondo, alla fine, non è quella di chi vi si adatta, ma di chi vi si inserisce. È la posizione di chi lotta per non essere soltanto oggetto ma anche soggetto delle Storia. [...] L'educazione come processo si fonda sull'incompiutezza dell'essere che si riconosce come tale. Donne e uomini diventano educabili nella misura in cui si riconoscono incompiuti» (Freire 2004, p. 47). 
fondamentalmente inedite e imprevedibili ${ }^{4}$, dell'esistenza umana per l'esistenza umana.

In ragione di questa lettura dell'esperienza, la pedagogia disegna un profilo di persona che la restituisce come attitudine ad agire «come principio di cambiamento» (Nosari 2017 , p. 23). È nella cornice di questo profilo che la persona può essere riconosciuta e affermata in quanto capace di "rilanciare" e di "misurare".

\section{La capacità di rilanciare}

All'interno di una processualità intenzionalmente trasformativa, il pensiero pedagogico pensa la persona come caratterizzata - prima di tutto - dalla capacità di rilanciare. Versione rivisitata dell'arendtiana capacità di «dare inizio» (Arendt 1997, p. 128), la capacità di rilanciare mostra e dà prova dell'approccio progettuale con il quale la persona si relaziona al cambiamento. Si tratta di un approccio marcato dalla cura e, di conseguenza, dall'attenzione e dalla decisione, che fa della persona una modalità (d'essere, di pensare e di agire) mai neutrale, mai indifferente, immancabilmente propositiva (Granese 1993; Fadda 2002). Proprio nel rilancio è custodita - custodia di cui si fa garante l'agire educativo - la possibilità di "aumentare" la portata del cambiamento. La persona è però capace di rilanciare secondo due accezioni diverse. Rilancia alla ricerca di un ulteriore traguardo che le permetta di ottenere sempre nuovi successi. In questa prima accezione, il rilancio ricerca e compie un cambiamento che si somma a quelli precedenti e che, inevitabilmente, verrà superato dal rilancio successivo. I grattacieli e le torri più alte del mondo ben rappresentano questa accezione del rilancio. Qualsiasi sia la costruzione indicata a rappresentare il traguardo massimo raggiunto, il titolo di "più alta del mondo" è assegnato nella consapevolezza del suo superamento. Si tratta dunque di un rilancio che implica una competizione per il raggiungimento di un

4. Non si tratta della ricerca del nuovo per il nuovo. Nello stesso tempo, l'interesse pedagogico non insegue il cambiamento per stra-volgere. Limmagine che potrebbe ben rappresentare il senso della trasformazione è quella dell'Arciere di stelle, opera d'arte di Riccardo Dalisi (2018), che lancia l'esperienza esistenziale verso prospettive che non sono già contenute nell'ordinario corso del cambiamento. 
L'«attitudine persona» o della capacità di trasformare traguardo sempre ulteriore ${ }^{5}$ : un traguardo il cui valore ha inevitabilmente una durata e, quindi, una scadenza.

Non è tuttavia questa l'accezione di rilancio con cui la pedagogia argomenta a favore di quel profilo di persona che la distingue e la ricerca per la sua attitudine ad agire come principio di un cambiamento trasformativo.

Il rilancio che mette la persona nelle condizioni di riconfigurare il cambiamento, apponendogli trame imprevedibili e inedite, non misura il cambiamento su una sequenza lineare di traguardi (in gioco non c'è semplicemente la sostituzione di una realtà con un'altra), ma lo unifica dandogli una direzione di senso che non si incontra unicamente alla fine in un (incerto quanto improbabile) progressivo avvicinamento. La persona, nel rilanciare, «non si rassegna, spera, si proietta verso l'inedito e il non-ancora, nonostante determinismi e ostacoli di diversa natura» (Rossi 1999, p. 6). Ne segue un rilancio "inesauribile" la cui inesauribilità non ha nulla a che fare con la produttività ${ }^{6}$, perché è espressione della capacità di aspirare che caratterizza l'essere umano in quanto persona, ossia di quella capacità che delinea l'orizzonte di senso all'interno del quale è possibile dare significato, concretezza e sostenibilità a tutte le altre capacità (Appadurai 2011).

5. Questa accezione di rilancio, grazie al progresso che ha caratterizzato soprattutto gli ultimi decenni, ha consegnato al cambiamento i tratti dell'accelerazione: «What does its mean for society to accelerate? We all notice that events around us seem to take place faster all the time. What was experienced as being extraordinarily speedy just yesterday now seems extraordinarily slow. Even basic life activities appear to be speeding up: fast food, fast learning, fast love. Perhaps, most significant, the time we're allowed to concentrate exclusively on one thing is progressively diminishing» (Rosa e Scheverman 2009, pp. 1-2).

6. La prova dell'inesauribilità del rilancio non va ricercata in argomentazioni necessariamente teoriche. Ne dà prova - sentimentale, ma non meno certa - il correre: «La maratona è un'avventura che si rinnova ogni volta. Ha sempre le stesse regole e pone chi la corre di fronte agli stessi problemi fisiologici e agonistici: ma quando lo starter spara il colpo di pistola inizia ogni volta una storia diversa, irripetibile, sorprendente. Si può essere preparati alla perfezione e fallire; più di rado si possono scoprire risorse di energia e determinazione che non si sospettava di possedere. Capita di passare in pochi minuti da un senso di orgogliosa sicurezza a qualcosa di molto simile alla disperazione; ma capita anche di sentirsi scivolare via di dosso un peso, ritrovare leggerezza, entrare in una dimensione quasi immateriale, in cui il corpo attraversa lo spazio con una quantità apparentemente minima di sforzo. Capita di sentirsi corridori perfetti, e dimenticare ogni dolore. Fino al traguardo, a volte. Oltre il quale iniziamo sempre un'altra storia e un'altra preparazione» (Breccia 2018, p. 30). 


\section{La capacità di misurare}

La capacità di rilanciare non è tuttavia sufficiente per promuovere una trasformazione che sia autentica espressione dell'umano. Lo sguardo pedagogico che disegna il profilo della persona, a sostegno della possibilità di un cambiamento che sia realmente occasione di una narrazione di senso, non può non riconoscere come indispensabile la sua capacità di misurare.

Anche la misura, tuttavia, può essere interpretata in due modi. Può essere ricondotta all'idea di obiettivo. In questo caso, rimanda alla capacità di verificare che i traguardi posti siano stati raggiunti. La misura come verifica poggia su criteri oggettivi: in quanto tale, può intervenire per correggere e per compensare affinché si arrivi all'obiettivo prefissato.

La capacità di misurare che lo sguardo pedagogico richiede alla persona per agire sul cambiamento non va confusa con questa capacità di "sovrapporre" il risultato raggiunto con quello atteso. Essa, proprio perché coinvolta in una trasformazione di senso, è data dalla capacità di valutare la direzione da dare al cambiamento. Tale valutazione - lontano dall'opinione o dalla preferenza (Ricœur 2005) - implica una presa di posizione con cui la persona si espone a favore di una chiara e netta direzione (non di altre). Si tratta, pertanto, di una misurazione necessariamente soggettiva che ha il proprio punto di forza proprio nella sua soggettività. Non c'è calcolo né verifica che possano indicare la direzione di senso: la decisione è solo della persona. In quanto tale, questa misurazione richiede alla persona una continua conferma da cui ci si attende non tanto un'attestazione distaccata, ma una partecipazione convinta.

"Incrociata" con questa capacità di misurare, la capacità di rilanciare non può non confrontarsi con l'idea di limite. All'interno dello sguardo pedagogico (che questo contributo propone), il rimando non è al limite inteso come "contenuto" che anticipa e condiziona la persona e il suo rilanciare, ma al ricœuriano "senso del limite" (Ricœur 1997). È infatti necessario che, in questa sua straordinaria capacità di cambiamento, la persona sia consapevole (o, meglio, prenda consapevolezza) del senso del limite con cui deve operare all'interno del proprio progetto di cambiamento. La necessità - prima di qualsiasi argomento ontologico 
L'«attitudine persona» o della capacità di trasformare o etico, antropologico o politico - è data e giustificata da un'esigenza sentimentalmente vissuta: in assenza di questo senso del limite, il rischio - di cui la storia ha già dato e continua a dare prova - è che valga indifferentemente qualsiasi rilancio, che quindi qualsiasi cosa diventi possibile e che, di conseguenza, tutto sia accettabile e nulla diventi intollerabile ${ }^{7}$.

\section{Le azioni della persona}

Il profilo così delimitato e distinto è da pensare in-azione. La proposta dello sguardo pedagogico, infatti, perderebbe di significato (e di potere) trasformativo, se non comprendesse anche le azioni che "traducono" tali capacità in concrete operazioni di cambiamento. Pertanto, nel riconoscere alla persona le capacità di rilanciare e di misurare, tale proposta le attribuisce anche due azioni fondamentali: l'azione del mantenere e l'azione dell'operare.

Per poter riconfigurare il cambiamento, la persona deve mantenere la direzione. In questa azione, la persona è impegnata in un'azione di resistenza. La possibilità di trasformare la nostra esistenza in un'esistenza orientata a una direzione di senso umano è unicamente affidata - come sentenzia la lezione di Stephane Hessel - a un'azione di resistenza (Hessel 2011). Il cambiamento che l'attitudine della persona alla trasformazione è in grado di portare è - di fatto - possibile soltanto attraverso un'azione che agisca come una sorta di forzatura su quello che potrebbe essere il corso naturale (o indifferente!) del cambiamento.

Attraverso questa azione di resistenza, lo sguardo pedagogico consegna un'immagine di persona compromessa in una militanza che la richiama alla responsabilità verso il compito della testimonianza: una testimonianza possibile solo attraverso un continuo "gesto di sfida" compiuto in opposizione a un cambiamento, altrimenti consegnato a un seguito senza altre possibilità (Ricœur 1989, p. 349);

7. “È intollerabile!" non è il grido dell'intollerante: «Non so più quale gerarchia stabile di valori può guidare le mie preferenze; il cielo delle stelle fisse si oscura. Dirò ancora: non distinguo più chiaramente i miei amici dai miei avversari $[. .$.$] . Non so più qual è il$ mio posto nell'universo, non so più quale gerarchia stabile di valori può guidare le mie preferenze, ma c'è per me dell'intollerabile» (Ricour 1997, p. 31). 
una testimonianza, pertanto, che non ammette astensioni o deleghe.

Di conseguenza, l'azione di resistenza con cui la persona mantiene la direzione di senso che intende dare al cambiamento la impegna in un continuo operare in-situazione. Questa opera, non riconducibile all'esecuzione di un programma dato, è chiamata a interpretare la direzione di senso secondo le opportunità o le condizioni che la situazione specifica presenta e offre. Nell'imprevedibilità e nell'irripetibilità delle situazioni, di fatto, si creano degli spazi per possibili altre direzioni: proprio questi spazi consegnano alla persona il compito di un'interpretazione "fedele" della direzione di senso riconosciuta e ricercata, un'interpretazione inevitabilmente soggetta a rischio di fraintendimento e di fallimento (Musaio 2007) tanto quanto aperta a una libertà segnata da sincerità e coraggio (Corsi 2006).

\section{La promozione della persona}

Per quanto le narrazioni pedagogiche possano essere state e continuino a essere diverse tra loro, in tutte - anche alla luce degli eventi storici più recenti - è possibile riconoscere una trama convergente che riconosce e afferma l'idea di persona come attitudine trasformativa.

Proprio in quanto lettura pedagogica, tale riconoscimento va tradotto in un'azione educativa volta a trasformare l'essere umano da "attitudine persona" a persona che agisce in situazione proprio come persona. Lo sguardo pedagogico, per l'implicita intenzionalità educativa, non può pertanto rinunciare a esercitare l'essere umano in quelle prove che più di altre promuovono la persona in quanto tale.

Quello che si potrebbe tracciare, allora, è un percorso che - seguendo la linea interpretativa di Gaetano Mollo (1996, p. 29) - marca una "via del senso". Lungo questa via, è possibile riconoscere tre particolari prove che permettono alla lettura pedagogica di provare l'essere umano nel suo agire come persona.

La prima è una prova di convinzione. Soltanto in ragione di una convinzione una persona può mantenere e resistere in quella direzione di senso che vuole dare al cambiamento. La convinzione è indispensabile perché, in sua mancanza, molto facilmente la persona sarà portata a delegare o a astenersi. Unicamente in presenza di una ferma e radicata 
L'«attitudine persona» o della capacità di trasformare convinzione (ma mai chiusa o sorda), la persona - di fatto - può mantenere e confermare la direzione nelle diverse situazioni in cui è coinvolta.

Per poter alimentare una convinzione, è però necessaria una seconda prova: quella che coinvolge la persona nella partecipazione a una causa. Il divenire persona è possibile unicamente all'interno di un'esperienza di partecipazione allestita per essere vissuta come continuum: un continuum che fa fare prova di partecipazione perché lega con ruoli e compiti che non prevedono livelli partecipativi diversi, ma coinvolgono tutti nella tutela, nella cura e nell'empowerment della comune cifra umana (Malavasi 2007).

La terza prova è quella che impegna la persona in una "causa grande" (Castoriadis 1998). La resistenza necessaria per mantenere la direzione di senso può essere esercitata solo se nella direzione di senso è riconosciuta una causa maggiore alla quale "sottomettersi". Tale sottomissione - come da lezione ricœuriana - non comporta alcun tipo di mortificazione o di negazione del valore della persona; al contrario, è l'occasione che più di ogni altra libera la persona in tutta la sua portata di senso.

Convinzione, partecipazione e impegno sono dunque le principali prove nelle quali esercitare un essere umano per trasformarlo in una persona che, non solo si presenti come portatrice di senso, ma agisca in nome di quel senso che riconosce come propria direzione. «Il valore della persona, pertanto, è sia nel suo essere che nel suo divenire. Dal lato ontologico-strutturale il valore è nella stessa dignità dell'essere umano in quanto tale; dal lato etico-esistenziale il valore è nella possibilità d'assunzione di compiti etici. All'educazione spetta la funzione di sollecitazione valoriale, volta a produrre interiori riscontri di senso» (Mollo 1996, p. 127).

$\mathrm{Ne}$ segue uno sguardo pedagogico che, nel proporre una soluzione alla "questione persona", si espone anche per una particolare idea di uomo, di società, di mondo, di presente, di passato e di futuro.

Bibiografia

Appadurai A.

2011 La capacità di aspirare: la cultura e i termini del riconoscimento, in Id., Le aspirazioni nutrono la democrazia, Etal, Milano. 
Arendt H.

1997 Vita activa. La condizione umana, Bompiani, Milano.

Bateson G.

1997 Una sacra unità. Altri passi verso un'ecologia della mente, Adelphi, Milano.

Bellingreri A.

2005 Per una pedagogia dell'empatia, Vita e Pensiero, Milano.

Bertin G.M., Contini M.C.

2004 Educazione alla progettualità esistenziale, Armando, Roma.

Breccia G.

2018 La fatica più bella. Perché correre cambia la vita, Laterza, Bari-Roma.

Castoriadis C.

1998 L'enigma del soggetto: l'immaginario e le istituzioni, Dedalo, Bari.

\section{Corsi M.}

2006 Il coraggio di educare. Il valore della testimonianza, Vita e Pensiero, Milano.

Fadda R.

2002 Sentieri della formazione: la formatività tra azione ed evento, Armando, Roma.

Freire $\mathrm{P}$.

2004 Pedagogia dell'autonomia. Saperi necessari per la pratica educativa, Edizioni Gruppo Abele, Torino.

Granese A.

1993 Il labirinto e la porta stretta, La Nuova Italia, Firenze.

Hessel S.

2011 Indignatevi!, Add, Torino.

Malavasi P.

2007 Pedagogia e formazione delle risorse umane, Pedagogia e Vita, Milano.

Mollo G.

1996 La via del senso, La Scuola, Brescia.

Musaio M.

2007 Pedagogia del bello. Suggestioni e percorsi educativi, FrancoAngeli, Milano.

Nosari S.

2017 Pedagogia del cambiamento, Utet, Torino.

Possenti V.

2013 Il nuovo principio persona, Armando, Roma. 
L'«attitudine persona» o della capacità di trasformare
Ricœur P.

1997 Muore il personalismo, rinasce la persona, in Id., La perso$n a$, Morcelliana, Brescia, pp. 21-36.

2005 Il giusto, vol. 1, Effatà, Cantalupa.

1989 Dal testo all'azione, Jaca Book, Milano.

Rosa H., Scheverman W.E.

2009 High-speed society. Social acceleration, power and modernity, Pennsylvania University Press, Philadelphia.

Rossi B.

1999 Tempo e progetto. Saggio sull'educazione al futuro, La Scuola, Brescia. 

Parte seconda.

Rileggere

grandi

pensatori

aA 



\section{Gesù divino e inclusivo di Paolo di Tarso. La ridefinizione di una identità personale Stefano Traini}

In questo intervento vorrei soffermarmi sul modo in cui Paolo di Tarso, nelle sue lettere raccolte nel Nuovo Testamento, ha (ri)costruito l'identità personale di Gesù. La questione è di enorme portata e alcuni specialisti hanno sostenuto l'ipotesi che Paolo abbia radicalmente modificato l'immagine del Gesù storico, diventando di fatto il vero artefice del cristianesimo ${ }^{1}$. Io proverò soltanto a isolare e analizzare alcune tracce testuali che da questo punto di vista sono particolarmente significative, sempre tenendo sullo sfondo i più importanti risultati delle ricerche sul Gesù storico $^{2}$. Se "persona" sottende il significato etimologico di

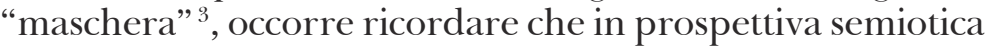
le identità personali si costruiscono nei testi, attraverso quei meccanismi di débrayage che sono stati analizzati da Benveniste e Greimas ${ }^{4}$.

1. Cfr. tra gli altri Schoeps (1961).

2. Sul Gesù storico si vedano Allison (1998); Crossan (1992; 1995); Meier (1991, 1994, 2001, 2009); Sanders (1993), Theissen e Marz (1996).

3. Cfr. la voce "persona” dell'Enciclopedia Treccani online. Sugli studi antropologici e psicoanalitici del concetto di persona cfr. Augé (1980).

4. Cfr. Benveniste (1939; 1956; 1958; 1970; 2009); Greimas e Courtés (1979). 
Comincerei con la credenza nella resurrezione di Gesù, che è il punto primo e centrale del pensiero di Paolo. Egli scrive: «Ma se Cristo non è risorto, vuota allora è la nostra predicazione, vuota anche la vostra fede» (1 Corinzi 15,14). La ridefinizione paolina dell'identità personale di Gesù parte da questo assunto, che quindi vale la pena specificare nel dettaglio. Nella concezione di Paolo, infatti, la risurrezione non coinvolge il corpo fisico e materiale, quanto piuttosto un "corpo spirituale". Paolo, come i Farisei e in generale molti Giudei del suo tempo, crede che alla fine dei tempi tutti i morti resuscitino con i loro corpi, ma specifica che si tratta di nuovi corpi spirituali. Siamo lontani dalla concezione greca di derivazione platonica secondo la quale l'anima immortale risorge in forma del tutto spirituale e disincarnata da un corpo fisico. Secondo Paolo il corpo fisico muore e alla fine dei tempi risorge in una nuova forma, in un corpo spirituale che è un nuovo modo di essere. Secondo Paolo, quindi, Gesù è risorto con il corpo, ma il corpo resuscitato è un "corpo spirituale", immortale:

Ciò che tu semini non prende vita se prima non muore. Quanto a ciò che semini, non semini il corpo che nascerà, ma un semplice chicco di grano o di altro genere. E Dio gli dà un corpo come stabilito, e a ciascun seme il proprio

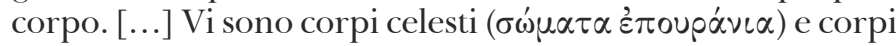
terrestri $\left(\sigma \omega \mu \alpha \tau \alpha\right.$ ध่ $\left.\dot{\omega}^{\prime} \gamma \varepsilon \iota \alpha \cdot\right)$, ma altro è lo splendore dei corpi celesti, altro quello dei corpi terrestri. [...] Così anche la resurrezione dei morti: è seminato nella corruzione, risorge nell'incorruttibilità; è seminato nella miseria, risorge nella gloria; è seminato nella debolezza, risorge nella potenza; è seminato corpo animale ( $\sigma \tilde{\omega} \mu \alpha \psi \psi \chi \chi \iota x o ́ v)$, risorge corpo spirituale ( $\sigma \tilde{\omega} \mu \alpha \pi \nu \varepsilon \cup \mu \alpha \tau \iota x o ́ v)$. (1 Corinzi 15, 35-44)

Il corpo risorto è quindi un corpo trasformato, spiritualizzato, e secondo Paolo la resurrezione di Cristo è la prova che è iniziato il processo cosmico di resurrezione dei corpi fisici in nuove forme spirituali ${ }^{5}$. Peraltro l'insegnamento di Gesù sulla resurrezione, conservato in Luca e secondo gli specialisti risalente alla fonte $Q$, enfatizza proprio una sorta di trasformazione in un corpo spirituale (angelicato), in

5. Tabor (2012, p. 62). 
II Gesù

divino e inclusivo di Paolo di Tarso

cui anche le distinzioni sessuali risultano cancellate: «I figli di questo mondo prendono moglie e prendono marito, ma quelli che sono giudicati degni della vita futura e della resurrezione dei morti, non prendono moglie né marito: infatti non possono più morire, perché sono uguali agli angeli e, poiché sono figli della risurrezione, sono figli di Dio» (Lc 20,34-36) ${ }^{6}$. Saranno gli evangelisti, qualche decennio dopo, a raccontare apparizioni di Gesù con il suo corpo terreno, probabilmente per dare più concretezza a un evento difficilmente credibile in ambienti ellenistici. Indicherei la credenza nella resurrezione come il primo atto nella riconfigurazione dell'identità personale di Gesù. Un atto decisivo che rimodalizza l'essere di Gesù e ridefinisce la sua dimensione somatica.

\section{Cristo fonte di salvezza}

Com'è noto, al centro della teologia di Paolo vi è la convinzione che la figura di Gesù abbia piena valenza soteriologica, cioè di salvezza: tutte le sue riflessioni sulla fede, sulla giustificazione e sulla resurrezione dipendono da questo presupposto. Per Paolo la figura di Cristo, con la sua resurrezione, consente di superare l'idea che la grazia di Dio passi attraverso comandamenti e precetti: dopo Gesù e grazie a lui, l'uomo può essere ritenuto "giusto" (cioè santo) non più in base a ciò che può fare di moralmente giusto in conformità ai dettami della Legge (Paolo parla di "opere"), ma in base alla fede nella morte e nella risurrezione di Gesù. In virtù di questa posizione Paolo richiede ai membri delle comunità da lui fondate non di diventare santi attraverso le opere, ma di mantenere la santità che è già presente in loro grazie alla fede in Cristo, e questo per mantenere uno status morale decoroso e all'altezza ${ }^{7}$. Il cambio di prospettiva è gravido di conseguenze: svincolare la salvezza (la giustificazione) dalla Legge, e quindi dall'imposizione esterna di

6. L'idea della resurrezione è presente nell'antica apocalittica giudaica ed è legata alla riproposizione di una vita fisica. Secondo la concezione ebraica di immortalità i morti vanno negli inferi e vi restano come ombre: alcuni risorgeranno alla fine dei tempi, dopo un giudizio di Dio, trasformati in nuovi corpi immortali. La nozione ebraica di resurrezione dei morti non prevede uno stato di beatitudine spirituale e "disincarnata", ma una vita in una nuova forma re-incarnata. Noi sappiamo che i Sadducei si opponevano all'idea di resurrezione, mentre i Farisei e gli Esseni la accettavano.

7. Cfr. 1 Cor 6,9-10; Lettera a Filemone 2,14-15; 1 Tessalonicesi 4,3-8. 
osservanze che dipendono dalla volontà umana, significa anche essere liberati dalla presunzione di costruire da soli la propria salvezza. Conta solo la fede in Cristo e l'esercizio dell'amore che ne deriva ${ }^{8}$.

Nella prospettiva ebraica la Legge resta in vigore fino alla venuta del Messia, quindi fino alla fine dei tempi. Con l'inaugurazione dell'éscathon la Torah dovrebbe concludere la sua funzione. Per contro Paolo sostiene che il Messia sia apparso prima della manifestazione escatologica del regno di Dio, cosicché ora la giustificazione - e quindi la salvezza - dipende solo dall'accettazione di Cristo e dalla fede in lui. Per Paolo, quindi, Cristo è il termine della Legge e la Torah è stata rimpiazzata dalla nuova Torah di Cristo:

Ma prima che venisse la fede, noi eravamo custoditi e rinchiusi sotto la Legge, in attesa della fede che doveva essere rivelata. Così la legge è stata per noi un pedagogo, fino a Cristo, perché fossimo giustificati per la fede. Sopraggiunta la fede, non siamo più sotto un pedagogo. Tutti voi infatti siete figli di Dio mediante la fede in Cristo Gesù. (Gal $3,23-26)$

Paolo non nega che il Dio di Israele abbia parlato per mezzo di Mosè e dei Profeti, ma insiste sul fatto che accanto al Dio di Israele vi sia il Signore Gesù, di fronte al quale l'intero universo si porrà in deferente ossequio. Quando Paolo parla della Torah di Cristo non pensa a un codice di norme, ma alla manifestazione del Cristo Spirituale in coloro che si sono uniti con Cristo attraverso il battesimo: Ebrei e non-Ebrei. Gesù è la persona che non rivela una nuova legge, ma porta una grazia che include tutti gli uomini, anche i Gentili. Si può dire che Paolo porti avanti una vera e propria battaglia in favore dell'inclusivismo. Paolo insiste sulla fede dirompente nel Cristo crocifisso e risorto e in quest'ottica si capisce anche la scarsa attenzione di Paolo per i contenuti missionari del Gesù terreno, visto che mai vengono citate da Paolo le parole di Gesù o le sue predicazioni. Lo scarto rispetto al Gesù storico sembra radicale. A proposito della Legge Gesù dice: «Non crediate che io sia venuto ad abrogare la legge o i profeti; non sono venuto ad abrogare, ma a compiere» (Matteo 5,17). In pratica Paolo

8. Cfr. Penna (2015, pp. 98-100). 
II Gesù

divino e inclusivo di Paolo di Tarso

ridefinisce l'identità messianica di Gesù Cristo. Per Paolo Gesù è il Messia (Christòs) e addirittura il Signore (Kyrios) che però non ha i connotati del sovrano potente e glorioso, ma di un condannato all'umiliazione della croce.

Vorrei far notare che questa visione paolina riconfigura il modello semiotico attanziale che emerge dalla vicenda del Gesù storico. Lì emerge infatti che Gesù, in quanto Destinante, indica al suo popolo (Destinatario-Soggetto) la via della salvezza (Oggetto di valore) da raggiungere attraverso un certo comportamento da tenere, con una maggiore attenzione alla sostanza rispetto alla forma. Si tratta quindi di un Programma Narrativo ben chiaro, basato su regole e comportamenti che Gesù espone nella sua predicazione attraverso racconti e parabole. Qui Paolo pare configurarsi come una sorta di Neo-Destinante che indica a un popolo molto più vasto (Giudei e Gentili) una diversa via per la salvezza: non il rispetto di comandamenti e precetti, non la Legge, ma la Fede in un ex-Destinante-Gesù del tutto riconfigurato: risorto in un corpo spirituale - quindi rimodalizzato - e pienamente divino. Nella visione di Paolo la fede porterà all'unione mistica con Cristo, cosicché l'umanità e Gesù diventano due attanti in sincretismo. I rituali del battesimo e dell'eucarestia vengono completamente ripensati in quest'ottica. Il battesimo nella visione di Paolo produce un'unione mistica con il "corpo spirituale" di Cristo ed è l'atto attraverso il quale si riceve lo Spirito Santo. E l'eucarestia diventa l'atto simbolico di mangiare e bere il corpo e il sangue di Cristo attraverso il pane e il vino: un rituale che ritroviamo nei vangeli proprio attraverso l'elaborazione di Paolo, espresso con un linguaggio che difficilmente può essere stato adottato dal Gesù storico ${ }^{9}$. Per Paolo mangiare il pane e bere il vino significa unirsi al corpo spirituale del Cristo glorificato nei cieli.

\section{Universalismo e inclusività}

Ma c'è di più. Paolo ritiene anche che Gesù sia l'iniziatore di una nuova stagione della storia caratterizzata da una ridefinizione dell'uomo, e - come abbiamo già accennato - la sua prospettiva è universalistica. A Paolo interessa l'uomo in

9. Cfr. Smith (2003). 
quanto tale, a prescindere da differenze etniche, culturali o religiose. Scrive ai Romani: «Io sono in debito tanto verso $\mathrm{i}$ Greci quanto verso i Barbari, tanto verso i sapienti quanto verso gli ignoranti» $(\mathrm{Rm} 1,14)$. E scrive ai Corinzi: «Mi sono fatto Giudeo per i Giudei, per guadagnare i Giudei. Per coloro che sono sotto la Legge - pur non essendo io sotto la Legge - mi sono fatto come uno che è sotto la Legge, allo scopo di guadagnare coloro che sono sotto la Legge. Mi sono fatto debole per i deboli, per guadagnare i deboli; mi sono fatto tutto per tutti, per salvare a ogni costo qualcuno. Ma tutto io faccio per il Vangelo, per diventare partecipe anch'io» (1 Cor 9,20-23). Gesù Cristo per Paolo rappresenta il superamento della disuguaglianza tra Giudei e Gentili, i quali ora possono essere equiparati. Paolo compie la sua attività missionaria con l'obiettivo principale di eliminare la distanza che separa i Gentili dai Giudei, considerati il popolo dell'alleanza con Dio, in un'ottica inclusiva rispetto agli "altri", ai "lontani", ai "diversi". Quindi se da un lato Paolo continua a riconoscere lo status proprio del popolo ebraico e la sua funzione storico-salvifica, dall'altro si impegna in un progetto di superamento della separatezza dai Gentili, irrinunciabile per Israele per salvaguardare la propria identità nazionale e religiosa. Nell'iscrizione greca posta nel Tempio tra il cortile dei Gentili e i cortili più interni riservati agli Israeliti si legge: «Nessuno straniero penetri al di là della balaustra e della cinta che circonda lo hierón; chi venisse preso in flagrante sarà causa a se stesso della morte che ne seguirà» (Orientis Graeci Inscriptiones Selectae 598). Nella lettera di Aristea del II secolo a. C. si legge che Mosè «ci ha circondati con una trincea invalicabile e con mura di ferro, perché non ci mescolassimo minimamente con gli altri popoli». E invece Paolo scrive: «Egli (Cristo) ha fatto di due una cosa sola, abbattendo il muro di separazione che li divideva, cioè l'inimicizia, per mezzo della sua carne. Così egli ha abolito la Legge, fatta di prescrizioni e di decreti, per creare in se stesso, dei due, un solo uomo nuovo, facendo la pace, e per riconciliare tutti e due con Dio in un solo corpo, per mezzo della croce, eliminando in se stesso l'inimicizia» (Ef 2,1416). Il messaggio di inclusività è radicale. $\mathrm{E}$ in questo Paolo si oppone al cristianesimo primitivo di Gerusalemme, che riteneva Cristo e la Torah del tutto compatibili. Negli Atti Giacomo dice a Paolo: «Tu vedi, fratello, quante migliaia 
II Gesù

divino e inclusivo di Paolo di Tarso

di Giudei sono venuti alla fede e sono tutti osservanti della Legge» (At 21,20). Del resto, quanto alla localizzazione geografica della predicazione Gesù dice ai suoi discepoli: «Non andate dai pagani, né entrate in una città di Samaritani. Rivolgetevi piuttosto alle pecore disperse della casa d'Israele» (Mt 10,5-6).

In termini semiotici possiamo dire che qui agisce a livello profondo l'asse semantico "inclusività" vs "esclusività" e che mentre nel racconto del Gesù storico viene privilegiato, con un investimento assiologico positivo, il termine "esclusività" - coerentemente con i valori dell'ambiente giudaico -, nel racconto paolino viene privilegiato il termine "inclusività". Questo nuovo investimento assiologico determina una riconfigurazione complessiva delle strutture narrative, discorsive e passionali. Non solo: ci accorgiamo che nella prospettiva di Paolo la ridefinizione della persona-Gesù implica una ridefinizione più complessiva dell'uomo, in senso inclusivo e universalistico. Paolo crede che gli spiriti risorti, di cui Cristo è il primo esemplare, andranno a costituire una sorta di famiglia cosmica nel regno di Dio. Si tratta di un'altra grande immagine di inclusione attorno alla figura di un Gesù che coincide ormai con Dio. Paolo pensa che Cristo abbia due tipi di filiazione, una terrena e una celeste: «nato dal seme di Davide secondo la carne, costituito Figlio di Dio con potenza secondo lo Spirito di santità in virtù della risurrezione dei morti» $(\mathrm{Rm} 1,4)$. Cristo dunque è l'immagine perfetta del Padre e il Padre riproduce l'immagine di suo Figlio in tutti coloro che partecipano alla sua filiazione. Questa trasformazione interiore avrà il suo culmine nella parusia. Dio, attraverso Gesù, ha cominciato a costituire una sorta di Famiglia Divina e Cristo - come "secondo Adamo" - è il primogenito di una famiglia di anime che si riconoscerà in lui. Infatti scrive: «chi si unisce al Signore forma con lui un solo spirito» (1 Cor 6,17). Il destino di questa Grande Famiglia Divina è quello di governare l'intero universo. Paolo concepisce il regno di Dio come l'acquisizione cosmica dell'intero universo da parte di questa Famiglia Divina composta dai Figli di Dio in Cristo: «se siamo figli, siamo anche eredi: eredi di Dio, coeredi di Cristo», dice Paolo nella lettera ai Romani $(8,17)$. E gli spiriti di questa Famiglia Divina (i "santi") parteciperanno con Cristo al giudizio finale, esercitando la propria funzione 
persino sugli angeli: «Non sapete che i santi giudicheranno il mondo? [...] Non sapete che giudicheremo gli angeli?» (1 Cor 6,2-3).

\section{La fine del mondo}

Nella predicazione di Paolo è centrale l'attesa della imminente fine del mondo con la parusia di Cristo. Egli è sicuro che lui e i suoi seguaci vedranno personalmente il ritorno di Cristo dai cieli. Crede che il mondo sia assai vicino a una radicale trasformazione. Ma sostenendo ciò si trova a gestire una contraddizione complessa: da un lato - in vista dell'imminente fine dei tempi - può relativizzare l'importanza delle attività quotidiane delle persone (sesso, alimentazione, ecc.); dall'altro deve fare in modo che la vita nelle comunità continui come se nulla di definitivo dovesse accadere. Chiede allora ai suoi seguaci di vivere come se la definitiva trasformazione spirituale fosse compiuta. Paolo cerca insomma di ricomporre il contrasto temporale tra un già e un non ancora. In alcuni passi Paolo parla di un "tribunale di Cristo" di fronte al quale tutti devono comparire; in altri passi Cristo sembra debba assumere un ruolo escatologico di liberatore o protettore, che sta dalla parte di chi crede in lui. La prima posizione sembra più vicina alla concezione giudeo-cristiana, mentre la seconda sembra più autenticamente paolina. Lidea di Paolo è che nel giudizio finale Gesù Cristo svolgerà un ruolo di intercessore. Al contrario dell'escatologia giudaica, che è esclusivamente orientata al futuro, quella "cristiana" di Paolo sostiene sorprendentemente che l'éschaton è già cominciato, e questa elaborazione escatologica influenzerà la dottrina dei decenni seguenti confluendo negli scritti evangelici. Nella Lettera ai Galati Paolo scrive: "Quando venne la pienezza del tempo, Dio mandò il Figlio suo, nato da donna, nato sotto la Legge, per riscattare quelli che erano sotto la Legge» $(4,4)$. Con la venuta del Figlio, quindi, è giunta la pienezza del tempo. Nella prima lettera ai Corinzi Paolo ricorda i peccati commessi dagli Ebrei nel deserto, e poi aggiunge: «Tutte queste cose però accaddero a loro come esempio, e sono state scritte per nostro ammonimento, di noi per i quali è arrivata la fine dei tempi». (1 Cor 10,11). L'attesa della fine viene poi attenuata, e il cambio di atteggiamento lo si coglie nelle lettere pseudoepigrafe, scritte dopo la morte di Paolo da suoi seguaci, 
II Gesù

divino e inclusivo di Paolo di Tarso

in un contesto critico in cui la fine dei tempi evidentemente tardava ad arrivare. Così nella seconda lettera alla comunità di Tessalonica Paolo espone una visione differente sulla fine dei tempi: «Vi preghiamo, fratelli, quanto alla venuta del Signore nostro Gesù e la nostra riunione con lui, a non agitarvi facilmente nel vostro animo, a non spaventarvi né da oracoli dello Spirito, né da parola o da lettera come spedita da noi, quasi che il giorno del Signore sia imminente» (2 Tes 2,1-2). Nelle lettere ai Colossesi e agli Efesini il punto focale del discorso non è più la fine dei tempi ma l'attuale signoria di Cristo, non solo a livello ecclesiale ma anche cosmico. Lo spostamento della fine dei tempi in un futuro indefinito non implica l'abbandono dell'interesse per la prospettiva escatologica. Basti ricordare i testi prodotti tra il I e il II secolo sull'escatologia futuristica come l'Apocalisse di Giovanni, le lettere di Pietro e di Giuda, e più tardi l'Apocalisse di Paolo e l'Apocalisse di Pietro, apocrife.

\section{Conclusioni}

Si sarà notato che la riconfigurazione identitaria di Gesù va di pari passo con la configurazione identitaria di Paolo. Chi è Paolo per porsi come una sorta di Anti-Destinante rispetto a Gesù? Quali sono le sue credenziali? Sappiamo che Paolo non ha mai incontrato Gesù, eppure concepisce se stesso come un apostolo e scrive alla comunità di Corinto: «Non sono forse un apostolo? Non ho veduto Gesù, Signore nostro?» (1 Cor 9,1). A proposito del vangelo, scrive ai Galati: «io non l'ho ricevuto, né l'ho imparato da uomini, ma per rivelazione di Gesù Cristo» (Gal 1,12). Nella seconda lettera ai Corinzi è scritto: «Se bisogna vantarsi - ma non conviene - verrò tuttavia alle visioni e alle rivelazioni del Signore. So che un uomo, in Cristo, quattordici anni fa - se con il corpo o fuori del corpo non lo so, lo sa Dio - fu rapito fino al terzo cielo. E so che quest'uomo - se con il corpo o fuori del corpo non lo so, lo sa Dio - fu rapito in paradiso e udì parole indicibili che non è lecito ad alcuno pronunciare» (2 Cor 12,1-4). Dunque Paolo equipara le visioni e rivelazioni di Gesù che ha avuto lui con la conoscenza diretta di Gesù avuta dai discepoli più stretti. Paolo dichiara di non aver compreso del tutto queste esperienze estatiche e spirituali, tuttavia è convinto che siano stati incontri con il Cristo. Da un lato Paolo non si ritiene degno di essere un apostolo per- 
ché per un periodo della sua vita ha perseguitato i cristiani: «Io infatti sono il più piccolo tra gli apostoli e non sono degno di essere chiamato apostolo perché ho perseguitato la Chiesa di Dio» (1 Cor 15,9). Dall'altro sottolinea che la sua chiamata è stata un evento singolare e straordinario che lo equipara agli altri apostoli: «Per grazia di Dio, però, sono quello che sono, e la sua grazia in me non è stata vana. Anzi, ho faticato più di tutti loro, non io però, ma la grazia di Dio che è con me. Dunque, sia io che loro così predichiamo e così avete creduto» (1 Cor 15,10-11). Più precisamente, Paolo crede di svolgere un ruolo cruciale nel piano cosmico attraverso cui Dio vuole portare la salvezza nel mondo attraverso Cristo. Addirittura crede di aver avuto la chiamata prima di nascere, quando era ancora nel seno della madre: «Ma quando Dio, che mi scelse fin dal seno di mia madre e mi chiamò con la sua grazia, si compiacque di rivelare in me il Figlio suo perché lo annunciassi in mezzo alle genti...» (Gal 1,15-16). Paolo crede di essere quindi in una posizione di primo piano nell'ambito della predicazione di Gesù e si ritaglia il ruolo di "apostolo dei Gentili", con l'obiettivo di estendere il messaggio di Cristo oltre i confini del mondo giudaico. Così come Cristo ha operato nel territorio di Israele, Paolo interpreta se stesso come un "secondo Cristo" che deve evangelizzare il mondo intero. Questa apertura è confermata nel seguente passaggio della lettera ai Romani: «Dico infatti che Cristo è diventato servitore dei circoncisi per mostrare la fedeltà di Dio nel compiere le promesse dei padri; le genti invece glorificano Dio per la sua misericordia, come sta scritto: Per questo ti loderò fra le genti e canterò inni al tuo nome» (Rm 15,8-9). Ma allora, come dicevamo, nella fase post-gesuana Paolo si impone come una sorta di Neo-Destinante: egli riqualifica ed espande il progetto originario di Gesù e indica a Ebrei e Gentili una diversa via per la salvezza che passa per la fede in un Gesù interamente ridefinito ${ }^{10}$. Ridefinendo Gesù, in definitiva, Paolo definisce se stesso, il suo ruolo e il suo progetto.

10. Il rapporto di Paolo con gli apostoli della Galilea è fondato su una sorta di "collaborazione conflittuale". Non si possono trascurare i legami e i debiti che Paolo ha nei confronti della chiesa primitiva protocristiana e dell'ebraismo. Per quanto riguarda la chiesa primitiva, emerge dall'epistolario la sua costante preoccupazione di mantenere legami solidi con i primi discepoli di Gesù (quindi con i "cristiani” Ebrei). Gli incontri a Gerusalemme attestano questo rapporto, così come alcuni riferimenti da cui si evince la 
Allison D.

1998 Jesus of Nazareth: Millenarian Prophet, Fortress, Minneapolis.

Augé M.

1980 "Persona", in Enciclopedia Einaudi, vol. 10, Einaudi, Torino, pp. 651-672.

Beilby J.K., Eddy P.R. (a cura di)

2009 The Historical Jesus: Five Views, InterVarsity Press, Downers Grove.

Benveniste E.

1939 Nature du signe linguistique, "Acta Linguistica», I (in Benveniste 1966, pp. 49-55; trad. it. 1971, Natura del segno linguistico, pp. 61-69).

1956 La nature des pronoms, in For Roman Jakobson, Mouton \& Co, The Hague - Paris (in Benveniste 1966, pp. 251257; trad. it. 1971, La natura dei pronomi, pp. 301-309).

1958 De la subjectivité dans le langage, "Journal de Psychologie», 55, pp. 257-265 (in Benveniste 1966, pp. 258266 ; trad. it. 1971, La soggettività nel linguaggio, pp. 310-319).

1966 Problèmes de linguistique générale, Gallimard, Paris (trad. it. Problemi di linguistica generale, il Saggiatore, Milano 1971).

1970 L'appareil formel de l'énonciation, «Langage», anno V, 17, pp. 12-18 (in Benveniste 1974, pp. 79-88; trad. it. 1985, Lapparato formale dell'enunciazione, pp. 96-106).

1974 Problèmes de linguistique générale, II, Gallimard, Paris (trad. it. Problemi di linguistica generale, il Saggiatore, Milano 1985).

2009 Essere di parola. Semantica, soggettività, cultura, a cura di P. Fabbri, Bruno Mondadori, Milano.

Crossan J.D.

1992 The Historical Jesus: The Life of a Mediterranean Jewish Peasant, HarperCollins, New York.

1995 Jesus: A Revolutionary Biography, HarperOne, New York (trad. it. Gesù: una biografia rivoluzionaria, Ponte delle Grazie, Firenze, 1994).

trasmissione di informazioni cruciali da parte dei primi seguaci di Gesù: «Vi ho trasmesso ciò che anch'io ho ricevuto, cioè che Cristo morì per i nostri peccati secondo le Scritture $\mathrm{e}$ che fu sepolto e che risuscitò il terzo giorno secondo le Scritture e che apparve a Cefa e poi ai Dodici» (1 Cor 15,3-5). 
1979 Sémiotique. Dictionnaire raisonné de la théorie du langage, Hachette, Paris (trad. it. con integrazioni Semiotica. Dizionario ragionato della teoria del linguaggio, a cura di P. Fabbri, Bruno Mondadori, Milano 2007).

Meier J.P.

1991 A Marginal Jew: Rethinking the Historical Jesus, vol. I, The Roots of the Problem and the Person, Doubleday, New York (trad. it. Un ebreo marginale: ripensare il Gesù storico, vol. 1, Le radici del problema e della persona, Queriniana, Brescia 2001).

1994 A Marginal Jew: Rethinking the Historical Jesus, vol. II, Mentor, Message and Miracles, Doubleday, New York (trad. it. Un ebreo marginale: ripensare il Gesù storico, vol. II, Mentore, messaggi e miracoli, Queriniana, Brescia 2002).

2001 A Marginal Jew: Rethinking the Historical Jesus, vol. III, Companions and Competitors, Doubleday, New York (trad. it. Un ebreo marginale: ripensare il Gesù storico, vol. III, Compagni e antagonisti, Queriniana, Brescia 2003).

2009 A Marginal Jew: Rethinking the Historical Jesus, vol. IV, Law and Love, Doubleday, New York (trad. it. Un ebreo marginale: ripensare il Gesù storico, vol. IV, Legge a amore, Queriniana, Brescia 2009).

Penna R.

2015 Paolo. Da Tarso a Roma, il cammino di un grande innovatore, il Mulino, Bologna.

Sanders E.P.

1993 The Historical Figure of Jesus, Penguin, New York (trad. it. Gesù: la verità storica, Mondadori, Milano 1999).

Schoeps H.J.

1961 Paul: The Theology of the Apostle in the Light of Jewish History, Westminster Press, Philadelphia.

Smith D.E.

2003 From Symposium to Eucharist. The Banquet in the Early Christian World, Minneapolis, Fortress Press.

Tabor J.

2012 Paul and Jesus, Simon and Schuster, New York.

Theissen G., Merz A.

1996 Der historische Jesus: ein Lehrbuch, Vandenhoeck \& Ruprecht, Göttingen (trad. it. Il Gesù storico. Un manuale, Queriniana, Brescia 1999, 2007³). 
Persona e comunità nel pensiero politico di Karol Wojtyła. Critica semiotica di un'analisi fenomenologica

Francesco Galofaro*

Applicando il metodo di analisi fenomenologica proposto da Edmund Husserl, negli anni Dieci Max Scheler ed Edith Stein lavorano alla nozione di persona per sviluppare un'indagine diretta allo studio scientifico dell'etica e della società, con esiti interessanti per ciò che riguarda la filosofia politica e del diritto. Il loro lavoro è ripreso nel secondo dopoguerra da Karol Wojtyła, nella sua veste di docente di etica all'Università Jagellonica di Cracovia e all'Università Cattolica di Lublino, allo scopo di aggiornare l'antropologia cattolica in opposizione a quella liberale e marxista. Alla base della formazione di comunità sociali e politiche vi è la struttura della sequela tra un maestro etico e i suoi seguaci. Le crisi politiche che portano alcune società a produrre alienazione e all'incapacità di promuovere l'autodeterminazione del proprio popolo sembrano accompagnarsi a leader non più 
in grado di incarnare ed esemplificare valori che possano essere co-sperimentati dalla comunità, secondo una relazione che Scheler chiama "intuizione emozionale", e che sottoponiamo qui al vaglio della critica semiotica. In questo senso, la crisi dell'Occidente contemporaneo può ben dirsi una crisi di significato.

\section{Il personalismo di Wojtyta}

Linteresse di Karol Wojtyła nei confronti della fenomenologia risale per lo meno agli anni del dottorato" . "Valutazioni sulla possibilità di costruire l'etica cristiana sulle basi del sistema di Max Scheler" (Wojtyła 1954) è una lettura delle opere di Max Scheler dedicate all'etica, in particolare Scheler (1913), nel tentativo di comprendere se la fenomenologia sia sufficiente a fondare l'etica cristiana e se non necessiti a propria volta di un'ontologia, che Wojtyła reperisce nel tomismo.

La prospettiva filosofica del futuro pontefice fu profondamente influenzata dall'amicizia con il fenomenologo polacco Roman Ingarden, allievo di Edmund Husserl a Gottinga, che lo introdusse fra l'altro al pensiero di Edith Stein. La filosofa precorre il tentativo di conciliare fenomenologia e scolastica che caratterizzerà gli scritti del Wojtyła maturo. Wojtyła ripagò il proprio debito intellettuale canonizzando la Stein nel 1998 e dichiarandola patrona d'Europa l'anno seguente. Da Ingarden e da Edith Stein Wojtyła eredita anche l'approccio realista, opposto all'idealismo.

Il fatto che il lungo corpo a corpo del trentaquattrenne Wojtyła con la filosofia di Scheler si sia concluso con un giudizio di "non autonomia" dell'approccio fenomenologico non deve trarre in inganno. In seguito, durante la docenza di etica all'università Jagiellonica di Cracovia e presso l'università di Lublino, Wojtyła ricorrerà proprio alla fenomenologia per aggiornare la proposta antropologica della Chiesa cattolica. Nella discussione critica che segue la pubblicazione di Persona e atto (Wojtyła 1969) l'abbandono del tomismo è un tema ricorrente ${ }^{2}$.

1. Molte sono le opere di carattere agiografico sulla vita di Giovanni Paolo II, ma manca una biografia intellettuale vera e propria. Possiamo rinviare il lettore alla notizia biografica contenuta in Reale e Styczen (2003, pp. 1515-1565), in forma di cronologia.

2. Un'ampia discussione fu pubblicata in «Analecta Cracoviensia» (1973), in lingua polacca. Si rimanda a Szostek (1973) per una sintesi in francese delle posizioni espresse dai 
Persona e comunità nel pensiero politico di Karol Wojtyła
Il legame tra il pensiero etico di Giovanni Paolo II e la fenomenologia è profondo: dal dibattito della fenomenologia realista il futuro pontefice trae la nozione di persona definita provvisoriamente come un'unità di atti di ogni genere. Non diversamente, Scheler (1913, p. 437), definisce la persona come «unità che sussiste in funzione del compimento d'atti sempre diversi in ragione della propria essenza, e nella misura in cui tali atti vengono pensati come compiuti». Quanto all'atto, Wojtyła (1969, p. 864) definisce in primo luogo l'actus humanus come un'azione volontaria che è espressione della coscienza, e in quanto tale è il luogo che ne manifesta le strutture. Il momento dell'operatività è presente nell'azione, assente in ciò che accade: l'esperienza dell'operatività rivela il rapporto causale tra persona e atto (Wojtyła 1969, p. 919). Certamente le premesse di Wojtyła si ritrovano in Scheler - basta pensare che «Persona e atto» è anche il titolo di un capitolo di Scheler (1913). Tuttavia, Wojtyła (1969) propone un "rovesciamento". Da un punto di vista morale e giuridico, solo la persona può compiere un atto: la nozione di atto presuppone la persona; è allora possibile uno studio scientifico della persona in quanto implicata dall'atto. In altre parole, attraverso gli atti studiamo le caratteristiche empiriche della persona, evitando la difficoltà di dover anticipare definizioni aprioristiche.

\subsection{La Chiesa cattolica tra liberalismo e marxismo}

Le ragioni per cui Wojtyła si rivolge alla fenomenologia si comprendono meglio nei Saggi integrativi che seguono la pubblicazione del suo opus majus, in risposta ai critici (cfr. Wojtyła 2003, pp. 1303-1511). In particolare, Wojtyła avverte l'assenza di una proposta etica, specificamente cattolica, di impegno civile e politico nel secolo in cui le grandi ideologie proponevano modelli totalizzanti di comportamento individuale. Difatti, da un punto di vista cattolico la proposta liberale è chiaramente inaccettabile, oltre che molto povera, basata com'è sull'egoismo individuale e null'altro; altrettanto problematica è la proposta comunista. In proposito, è interessante una nota al primo saggio, datato 1978 e dedicato alla relazione tra le classificazioni cosmolo- 
giche dell'uomo e a un'indagine sulla soggettività. Wojtyła definisce "cosmologico" quel tipo di comprensione fondato sul convincimento della riducibilità dell'uomo al mondo (Wojtyła 2003, p. 1319). Secondo Wojtyła, il materialismo dialettico opera una riduzione cosmologica perché vede l'antropogenesi come un processo che si dà attraverso il lavoro e che riduce l'uomo a un esito (Wojtyła 2003, p. 1319, n.2). È ben noto che, per Marx e per i suoi interpreti, la coscienza è il risultato dell'essere sociale ${ }^{3}$.

Wojtyła non è convinto da questo caso specifico di riduzione cosmologica, come da ogni altro tentativo a prescindere dalla prospettiva filosofica considerata: secondo la fenomenologia, la coscienza sarebbe caratterizzata da alcune strutture irriducibili che caratterizzano noi tutti in quanto soggetti che hanno esperienza di sé.

Wojtyła è peraltro consapevole del fatto che la filosofia scolastica, il cuore ideologico del cattolicesimo della sua epoca, è insufficiente a contrastare la riduzione cosmologica operata dal marxismo perché a propria volta opera riduzioni simili. Il fatto è che Aristotele definisce l'essere umano un animale razionale. Anche questa definizione riduce l'uomo al mondo indicandone il posto, la casella: il genere è l'essere vivente, di cui l'uomo è solo la specie dotata di comprendonio (noēsis). Anche la definizione aristotelica, dunque, non si spinge oltre l'individuazione della piccola casella occupata dall'uomo nel cosmo (Wojtyła 2003, p. 1320).

2.2 Personalismo e ontologia

Laltra fondamentale definizione di persona si deve a Severino Boezio: rationalis naturae individua substantia (PL 64, p. 1343). Anche in questo caso Wojtyła (2003, n. 4) non ne è soddisfatto: se qui non è presente il genere (animal) a esprimere la riduzione cosmologica, in questa seconda definizione l'uomo è accomunato a ogni altra cosa dal semplice essere sostanza, ovvero da una comune base metafisica. Tutte le riduzioni viste fin qui, in effetti, tentano di chiarire cos'hanno in comune gli uomini a prescindere dalla propria identità. Non

3. Pur ponendosi in netta opposizione al personalismo, Simone Weil (1955) rinfaccia un'aporia simile a Marx. Da un lato, infatti, contro ogni illusione ideologica il filosofo di Treviri constata materialisticamente che la realtà è completamente regolata da rapporti di forza; d'altro canto non è chiaro dove fondi il bisogno di giustizia. 
Persona e comunità nel pensiero politico di Karol Wojtyła possono soddisfare il filosofo morale perché non dicono nulla sul valore di ciascuno di noi.

Lo scopo di Wojtyła, comunque, non è opporre una più profonda comprensione della persona alla sua collocazione nel cosmo; nel saggio considerato egli chiarisce che la prima è un'interpretazione della seconda: se cerchiamo di comprendere meglio chi siamo è per capire il senso della nostra collocazione nel mondo. La definizione che Boezio dà di persona funge in Wojtyła (2003, p. 1321) da «portinnesto ontologico» per quella fenomenologica: un «terreno metafisico» che è condizione necessaria per la coltivazione e la realizzazione della soggettività personale sulla base dell'esperienza. Da qui Wojtyła muove per ulteriori indagini sullo specifico della soggettività essenziale all'uomo come persona, che egli identifica con l' «irréductible dans l'homme» (2003, p. 1318). Wojtyła cerca nell' esperienza un percorso di "conquista" dell'Io, in termini di autodeterminazione, autopossesso, autodominio: è il campo del "morale" (2003, p. 1325). Dunque, qui è in gioco un tipo diverso di conoscenza, rispetto a quella cosmologica. Infine, in chiusura del saggio, Wojtyła sottolinea come anche il campo dell'esperienza sia reale, collocando la sua indagine in una prospettiva realistica, in opposizione all'idealismo.

\subsection{Persona e comunità}

Il secondo saggio integrativo in realtà precede il primo (Wojtyła 1976) e affronta temi molto complessi quali l'immagine, l'intersoggettività, il vincolo dell'oggettività, la metodologia fenomenologica. Nella prima parte il nostro autore torna a ribadire il rapporto fra fenomenologia e ontologia scolastica, quasi a rassicurare i propri critici; nella seconda, comunque, sottolinea le gravi insufficienze che sono proprie dell'impostazione stessa della filosofia scolastica. Infatti, dal punto di vista morale, la realizzazione dell'uomo (autocoscienza, autodominio, autoteleologia ecc.) avviene nella comunità. Tuttavia, dal punto di vista metafisico che interessa la scolastica, l'appartenenza di un essere umano a questa o quella comunità è meramente accidentale. Nonostante questo, la comunità di appartenenza è il fondamento di quel che si può dire sulle persone: "il polacco", "il cattolico", "il cittadino", "l'operaio". Inoltre, che l'uomo sia o non sia alienato il suo essere non cambia, da un punto di vista metafisico. 
Wojtyła (2003, pp. 1379-1386) introduce qui l'opposizione partecipazione/alienazione. Partecipare all'umanità dell'altro implica esplicitamente una nozione di "umanità" del tutto diversa da quel che la scolastica avrebbe considerato un universale; al contrario, per Wojtyła si tratta di un io personale in ogni caso unico e irripetibile. Opposta alla partecipazione, l'alienazione costituisce una sorta di de-umanizzazione dell'umano. Questo è il genere di alienazione che caratterizza tanto le società liberali quanto quelle comuniste, ed è il bersaglio privilegiato del filosofo morale. L'individualismo conferisce alla persona la possibilità di realizzarsi nell'agire insieme con gli altri, contrapponendo il bene dell'individuo a quello di ogni altro individuo; paradossalmente, il "totalismo" (sic) parte da una concezione molto simile, presupponendo nel singolo solo la tendenza a perseguire il bene individuale, e pertanto mira a realizzare il bene comune limitandolo, basandosi sulla costrizione (Wojtyła 1969, pp. 1181-1186).

Qui si introduce - ed è cruciale - la distinzione tra comunità e società. Edith Stein (1922), vede la comunità come un soggetto collettivo universalista, in cui ciascuno è soggetto sulla base di un reciproco riconoscimento di diritti e doveri nella misura in cui sono condivisi i valori fondanti. In questo modo, la comunità stessa è un attore collettivo che incarna la funzione di soggetto ${ }^{4}$. Diversamente, nella società l'Altro è meramente uno strumento; il riconoscimento reciproco manca e così l'attribuzione di diritti e doveri risponde alla medesima strumentalità. Secondo Wojtyła, la società può sussistere anche senza comunità laddove i rapporti sociali sono fonte di alienazione.

2.4 Wojtyła interprete del marxismo

E interessante come Wojtyła generalizzi il fenomeno dell'alienazione, descritto da Marx, alle società comuniste. Wojtyła prende atto del carattere strumentale dei rapporti tra esseri umani nel capitalismo (per Marx, i lavoratori sono un fattore della produzione) rinvenendo la medesima strumentalità nella società comunista polacca del secondo dopoguerra, cui contesta l'incapacità di realizzare la persona umana dal punto di vista dell'autodeterminazione. E questo,

4. Per un'analisi semiotica della nozione steiniana di comunità, si veda Galofaro (2021). 
Persona e comunità nel pensiero politico di Karol Wojtyła non perché non riconosca l'efficacia del marxismo come filosofia politica, ma perché gli imputa una carenza come filosofia morale (la riduzione cosmologica). Wojtyła riscontra tuttavia nel cattolicesimo il medesimo punto debole; formula allora una nuova proposta, basata sull'analisi fenomenologica della persona, allo scopo di far prevalere il cattolicesimo sul marxismo.

Non deve sorprendere il fatto che Wojtyła, avversario del marxismo, ne sia anche un conoscitore non superficiale. Non era certo possibile ignorare il marxismo nel contesto storico del secondo dopoguerra, quando questa filosofia poteva ben dirsi vincente sul piano politico ed egemone su quello culturale, in particolare in Polonia: Wojtyła (2003, p. 1331) ammette che il materialismo marxista costringe la filosofia cattolica a rispondere a questioni che aveva tralasciato $^{5}$. Nel far questo, come si è visto, Wojtyła la rinnova, non solo recuperando la fenomenologia ma perfino incorporando concetti provenienti dal marxismo stesso.

\subsection{Il problema dell'Altro}

Un problema condiviso da tutti gli approcci fenomenologici è la relazione tra il Soggetto e l'Altro. Il terreno di indagine su cui si colloca la fenomenologia è un approfondimento del rapporto tra le cose e la coscienza che ne abbiamo, tesa a spiegare come sia possibile una conoscenza oggettiva del mondo pur partendo dai tanti punti di vista che ne abbiamo. È dunque inevitabile chiedersi cosa fa sì che il mondo-dal-punto-di-vista-mio sia valido anche dal-puntodi-vista-altrui, da un lato; dall'altro, perché consideriamo gli altri quali soggetti come noi, e non alla stregua di "cose" che arredano il nostro mondo. C'è anche chi, nel movimento fenomenologico, ha risposto alla seconda domanda considerando l'Altro come un mero strumento, come Martin Heidegger (1927), proponendo una semiotica per cui il senso stesso coincide con l'utilizzabilità. La contiguità

5. Può essere questa la ragione per cui, divenuto papa, Wojtyła (2005, pp. 25-28) mantenne una distinzione tra nazionalsocialismo e comunismo, definendo il primo "male assoluto", il secondo "male necessario". Wojtyła interpreta in chiave soteriologica il tempo concesso da Dio al comunismo in rapporto a quello dato al nazismo: "Succede infatti che in certe concrete situazioni dell'esistenza umana il male si riveli in qualche misura utile, in quanto crea occasioni per il bene". Il passo sembra ancora in grado di suscitare reazioni sopra le righe tra gli anticomunisti (cfr. Fantolino 2008). 
tra questo punto di vista e il nazionalsocialismo, per quanto dibattuta, è all'origine delle scelte filosofiche di una generazione: citiamo solo un'opera come Totalità e infinito, di Immanuel Lévinas (1961). Altri filosofi invece non vedono alcun rapporto tra il pensiero di Heidegger e le sue scelte politiche - ad esempio Derrida (1987) - e propendono per un'adesione strumentale, quando non per un'infatuazione passeggera ${ }^{6}$. Per tutto ciò che abbiamo scritto fin qui, nel caso di Wojtyła questo punto di vista filosofico sarebbe inaccettabile, e anzi finirebbe per causare alienazione, nella misura in cui esso divenisse ideologia ufficiale.

Come per ogni fenomenologo, anche secondo Wojtyła l'esperienza è sempre esperienza di qualcosa. Inoltre, poiché facciamo esperienza degli altri, l'uomo è contemporaneamente soggetto e oggetto di esperienza, entro la quale è collegato all'operare. Nell'argomento di Wojtyła gioca un ruolo importante la generalizzabilità agli altri dell'esperienza dell'io. Si tratta di un ragionamento simile all'induzione matematica ${ }^{7}$ :

6. Il primo avversario della concezione di Heidegger fu il suo maestro Husserl (1935). È noto che Husserl considerava la mera strumentalità delle relazioni umane riscontrabili nella società degli anni Trenta come un sintomo della crisi dell'umanità europea, mentre Heidegger avrebbe finito per aderire al nazismo. Al netto della contiguità filosofica, che Heidegger abbia avuto un ruolo politicamente attivo nella costruzione dello Stato nazionalsocialista è noto da tempo - si veda Ott (1993), Losurdo (1991). Stupisce rileggere, di quando in quando, della sua «freddezza nei confronti del regime a partire dal 1934»-Gorret (2019). Volpi (2009: capitolo x) ricostruisce la lotta che vide in competizione Heidegger, da un lato, e Alfred Rosenberg ed Ernst Krieck, dall'altro, per imporsi alla direzione dell'Archivio-Nietzsche negli anni dal '34 al '38. Inoltre, tra il '34 e il '36 Heidegger ricoprì un ruolo di primo piano nel Comitato per la filosofia della giustizia presso l'Accademia per il diritto tedesco (Ausschuß für Rechtphilosophie der Akademie für Deutsches Recht) della quale facevano parte gerarchi quali Hans Frank, Julius Streicher, Carl Schmitt e Alfred Rosenberg (Faye 2005). In tale contesto, le note satiriche su Rosenberg annotate sul proprio diario nello stesso periodo - Heidegger (2018), - che Gorret cita a riprova della propria tesi, vanno viste in realtà come testimonianza delle rivalità e delle lotte di potere interne alla kulturkampf nazionalsocialista. L'adesione al nazismo che contestiamo a Heidegger non è fideistica ma politica: egli vide nell'adesione al partito nazionalsocialista - dal quale non uscì fino al ' 45 - l'occasione per la propria Selbstbehauptung, nel nome della quale nessun compromesso morale gli è parso inaccettabile. Nel rapporto tra la nozione di utilizzabilità (1927) e la sua successiva interpretazione del pensiero di Nietzsche vanno cercate le basi filosofiche di una dottrina nazionalsocialista "alternativa" alla quale l'autore rinunciò solo perché negli anni Trenta non prevalse, e non perché si fosse improvvisamente pentito o ricreduto.

7. Base dell'induzione: la proprietà $\mathrm{P}$ vale per un insieme che conta un solo elemento $\mathrm{n}$; passo induttivo: $P(n)$ implica $P(n+1)$. Conclusione: $\mathrm{P}$ vale per un insieme con un qualunque numero di elementi. 
Persona e comunità nel pensiero politico di Karol Wojtyła base dell'induzione: io faccio esperienza di esistere e operare in modo diverso dagli altri uomini;

passo induttivo: questo può dirsi di ogni altro uomo;

conclusione: caratteristica che accomuna tutti gli uomini è la diversità di ciascuno di essi.

Per quanto possa suonare paradossale, la conclusione è perfettamente logica. Nella costruzione filosofica dell'immagine della persona-soggetto l'autore attinge senz'altro all'esperienza del suo Io, ma mai separatamente dagli altri o in contrapposizione agli altri (Wojtyła 2003, p. 1334). Questo passaggio sembra una presa di posizione sul metodo di una fenomenologia che voglia dirsi scientifica. Per Wojtyła l'oggettività dell'esperienza è un vincolo che viene violato quando si accettano nozioni come quella di "coscienza pura" o di "soggetto puro". Questo vincolo impedisce anche di parlare di strutture che caratterizzano l'uomo una volta per tutte, immutabilmente: il carattere empirico del sapere umano obbliga a reinterpretare costantemente l'immagine dell'uomo-persona. Sebbene la presa di distanza di Wojtyła nei confronti della nozione kantiana di ego trascendentale sia meno radicale di quella scheleriana (Wojtyła 1969, p. 856, n. 8), in questo passo possiamo cogliere non solo la fondamentale divergenza tra la fenomenologia realista, incarnata da Wojtyła, e quella trascendentale che caratterizza i prosecutori del "secondo Husserl", ma anche tra la prima fenomenologia e altre scuole anti-fenomenologiche, quali lo strutturalismo. Scheler rifiuta la nozione di ego trascendentale. Se per l'idealismo trascendentale il senso della parola "oggetto" collima necessariamente con la possibilità di identificazione di qualcosa per mezzo di un io, secondo Scheler l'identità è una caratteristica essenziale dell'oggetto, e la relazione tra io e oggetto non è univoca, ma reciproca (Scheler 1913, p. 462). Inoltre, Wojtyła (1969, p. 854, n. 4) rifiuta anche i metodi della fenomenologia trascendentale, perché l'epoché comporta la separazione tra essenza ed esistenza attuale.

\section{Max Scheler tra fenomenologia e semiotica}

Senza dubbio, il saggio di Wojtyła sul rapporto Ego/Alter dimostra in maniera brillante una posizione che era già stata proposta da Max Scheler: siamo già-da-sempre par- 
te una collettività ed è un problema del nostro processo di formazione e di crescita il distinguere le nostre idee da quelle altrui. Si tratta di una posizione ancora molto attuale in filosofia: l'individuazione avviene a partire dal collettivo, ed è un processo che non si conclude mai realmente ( $\mathrm{Si}$ mondon 2005).

Di origini ebraiche, Scheler divenne un punto di riferimento per il cattolicesimo tedesco sia per le posizioni politiche pacifiste, sia per i contenuti della sua indagine sulla religione. Non si convertì tuttavia al cattolicesimo e nell'ultima fase della sua indagine mutò opinione sul problema del dio personale delle religioni monoteiste (cfr. Spiegelberg 1971, pp. 262-263). Qui di seguito ci occupiamo di alcuni aspetti del suo studio scientifico sull'etica costitutivi del legame sociale (Scheler 1913) riletti da Wojtyła (1954), i quali presentano nessi interessanti col dibattito semiotico attuale.

\subsection{Il valore}

Allievo di Franz Brentano, Max Scheler abbracciò il metodo fenomenologico di Husserl che applicò a uno studio scientifico dell'etica in un senso materiale. Il suo obiettivo polemico era il formalismo della morale kantiana, incapace di rendere conto dei contenuti dell'esperienza morale, ovvero dei valori. Questa caratteristica della sua ricerca attrasse Wojtyła fin dai primi passi della sua ricerca.

La teoria del valore proposta da Scheler (1913) è di grande interesse semiotico, per le sue analogie con la nozione di valore propria della semantica strutturale e della semiotica di Greimas e Courtés $(1979 \text {, p. } 375)^{8}$, in particolare relativamente ai valori timici. Secondo Scheler, tutti i va-

8. Vi sono anche differenze notevoli e vanno sottolineate. Infatti, nonostante le analogie nella struttura degli oggetti colti dai rispettivi punti di vista, la fenomenologia non offre un fondamento per la semiotica, al punto da consentirci di ridurre la seconda alla prima. Coerentemente con l'approccio della fenomenologia realista, l'indagine di Scheler si pone a un livello empirico (è il ritorno alle cose stesse, secondo l'espressione di Husserl). La "cosa" cui Scheler vuole ritornare è il valore, che egli considera come l'oggetto dell'esperienza emotiva. Per giustificare questo passo, occorre tenere a mente il postulato metodologico della fenomenologia realista, secondo cui ogni esperienza ha un correlato reale. Questo porta ad esiti apparentemente paradossali: se diverse persone intorno a un tavolo dichiarano di avere un'esperienza telepatica, questa deve aver un correlato oggettivo - anche se non è detto coincida con ciò che ne pensano i nostri "telepati” (cfr. Ales Bello, Pellegrino 2014). All'opposto del realismo, come semiotico sarei solo obbligato a pensare che tale esperienza abbia un significato condiviso per i partecipanti, un significato che è possibile analizzare scientificamente. 
Persona e comunità nel pensiero politico di Karol Wojtyła lori - compresi "buono" e "cattivo" - sono qualità materiali ordinabili secondo un rapporto di superiorità e inferiorità e indipendenti dalla forma d'essere in cui si presentano, considerate come mero supporto (Scheler 1913, p. 38). "Buono" e "cattivo", inoltre, sono due valori speciali, perché ci permettono di ordinare gli altri (1913, p. 47). Il valore è la prima cosa che cogliamo negli oggetti. Tra i suoi esempi, è interessante quello della degustazione del vino: prima cogliamo il valore del vino, e solo in seguito passiamo a interrogarci sulla sua composizione, sulla provenienza del vitigno, sul metodo di imbottigliamento. La prima cosa che cogliamo è dunque una sfumatura assiologica. Il valore è un protofenomeno, la condizione di possibilità che ci consente di cogliere il fenomeno stesso, il quale è peraltro un puro e semplice medium. Il valore non è una qualità essenziale del fenomeno né un suo accidente; permette al fenomeno di venire alla luce: il valore è il «primo messaggero dell'oggetto» (Scheler 1913, p. 39). Proprio come per la semiotica narrativa, non si danno valori che non siano investiti in oggetti; d'altra parte, gli atti dei soggetti non mirano a ricongiungersi semplicemente con certi oggetti, quanto piuttosto coi valori investiti in essi. Come semiotici, non consideriamo il valore come un fatto di pertinenza metafisica, e in questo Scheler ci ha preceduti: il valore è Objekt, non Ding. Tuttavia, in Scheler sembra mancare una nozione autonoma di semantica. Certamente, come nota Wojtyła (1954), da un lato il valore non ha a che vedere con la struttura ontica della cosa, ma con una "nuova forma"; d'altra parte, per Scheler questa "seconda forma" è ciò che la cosa riceve nella percezione affettiva intenzionale, che per lui coincide con la conoscenza intuitiva emozionale. Una nozione che desta più di una perplessità, e che sottoporremo più oltre a critica semiotica.

\subsection{Un percorso passionale}

Se non troviamo in Scheler una semantica, è piuttosto interessante constatare la presenza, nel suo pensiero, di una semiotica delle passioni e perfino un interessante percorso passionale, tema di riflessione tra i semiotici durante gli anni Novanta. Secondo questo punto di vista, i valori si presentano nell'esperienza fenomenologica come contenuti della vita emozionale umana. Sono i dati dell'esperienza 
emotiva. Vi sono analogie e differenze con l'impostazione di Greimas e Fontanille (1991) perfino per quanto riguarda la nomenclatura della sintagmatica proposta.

Nella semiotica dell'ultimo Greimas, fare ed essere danno luogo a due percorsi canonici distinti, e si trovano a un medesimo livello gerarchico: con Hjelmselv (1943) diremmo che sono due costanti. Non troppo chiari sono i rapporti tra i due percorsi, nonostante siano ricalcati l'uno sull'altro (cfr. Bertrand 2000, p. 2002). In Scheler, come del resto in Wojtyła, il fare presuppone l'essere, ed è questo il motivo per cui il secondo può essere indagato a partire dal primo, ovvero dall'atto che lo manifesta.

Scheler (1913, p. 127) distingue pertanto sette elementi nell'atto: (1) la presenza della situazione e dell'oggetto dell'atto; (2) il contenuto che deve essere realizzato dall'atto; (3) il volere questo contenuto e i suoi gradi, che portano dalla disposizione, attraverso l'intenzione ${ }^{9}$, la deliberazione e la risoluzione (Vorsatz), fino alla decisione; (4) la classe di attività dirette verso il corpo vissuto (un voler fare, "essere disposto a fare"); (5) gli stati di sensazione e i sentimenti collegati a queste attività; (6) la realizzazione del contenuto nell'esperienza (la "performance"); e (7) gli stati e i sentimenti posti dal contenuto realizzato.

Sottolineiamo alcune caratteristiche di questa semiotica passionale, posta al cuore dell'etica di Scheler: al punto (3) sono tratteggiate le fasi di un percorso passionale dell'essere che sfociano nel voler fare. Inoltre, anche nella performance e nella realizzazione abbiamo un "riempimento" di contenuti passionali; infine, la decisione di agire dipende per Scheler da un processo interiore che riguarda l'essere del soggetto, non un qualche rapporto con un secondo soggetto che funge da attante manipolatore (colui che fa fare).

Non vogliamo proporre il percorso passionale-narrativo descritto da Scheler come sintesi dei rispettivi percorsi proposti dalla semiotica; si direbbe, tuttavia, che qualunque

9. En passant notiamo - con Wojtyła (1954, pp. 274-278) - che l'intenzionalità in Scheler differisce leggermente da quella husserliana e tomista: l'intentio è legata alla volontà e all'intelletto, mentre in Scheler l'uomo conosce tramite percezioni affettive. Non tutte le emozioni sono intenzionali, bensì solo quelle più elevate. La vita emozionale consiste in una stratificazione che dipende dalla gerarchia di valori di cui ci occupiamo nel paragrafo seguente. Le esperienze emozionali si danno soprattutto nello strato spirituale, gerarchicamente più elevato. 
Persona e comunità nel pensiero politico di Karol Wojtyła modello tenti di spiegare il significato manifestato dalle azioni debba rendere conto del senso dell'azione stessa in relazione agli oggetti e ai corpi implicati, che la fenomenologia descrive in termini di "riempimento".

\subsection{La preferenza}

Per Scheler (1913, pp. 141-148) i valori costituiscono una gerarchia dell'ordine del "preferire". Tale gerarchia per Scheler è reale: lo studio scientifico dell'etica ha lo scopo di farla emergere. Ciascun livello della gerarchia presenta un proprio sistema di categorie assiologiche, che ne definiscono l'orientamento:

1) sentire sensibile: godere/soffrire, gradevole/sgradevole;

2) valori vitali: salute/malattia, vecchiaia, morte; ascesa/ decadenza; esaurimento/esuberanza; troviamo qui anche buono/cattivo in quanto legati alla sfera del fare (ad esempio nel giudizio "è un buon artigiano");

3) valori spirituali: estetici (bello/brutto); valori che fondano gli ordinamenti giuridici; valori della conoscenza pura, non finalizzata al dominio dei fatti naturali (filosofia); atti soggettivi legati a questi valori: stimare/disistimare, simpatizzare/aborrire; approvare/disapprovare, ecc.

4) valori religiosi: sacro/profano; la vicinanza/lontananza dal sacro determina la felicità/infelicità della vita vissuta. La realizzazione di questo valore implica l'amore rivolto alle persone.

Ricordiamo che per Hjelmslev (1943) "gerarchia” è sinonimo di "semiotica"; tuttavia, in Scheler manca la consapevolezza di un ordine semiotico autonomo rispetto alla dicotomia soggettivo/oggettivo. Di conseguenza, Scheler (1913, pp. 43-45) ragiona così: poiché i "valori" non dipendono dalle scelte individuali, non possono che essere dell'ordine del reale.

Si potrebbe ironizzare su questa gerarchia, che ricorda il marketing e la piramide di Maslow (1954). Più serie le critiche di Wojtyła (1954, pp. 340-341): egli nota che non sempre si sacrifica un valore inferiore a uno superiore (dare la vita per la patria); accade anche il contrario (interrompere gli studi per motivi di salute). Che la storia, e il progresso dei maestri di morale portino l'etica sociale ad "aggiustarsi" è la scommessa di Scheler (1913, p. 377), avversario del relativismo; che tale convinzione sia poco fondata è l'opinione 
di Wojtyła, non perché quest'ultimo fosse un relativista ma perché convinto della necessità della rivelazione per orientare scelte morali che parrebbero equivalenti dal punto di vista della "preferibilità":

La sola posizione gerarchica di un determinato valore data nella percezione affettiva intenzionale come sua "superiorià" o "inferiorià" non basta a definire il valore morale oggettivo dell'atto interno o esterno, in cui il soggetto personale realizza un adeguato valore oggettivo col volere o con l'azione. (Wojtyła 1954, p. 341)

$\mathrm{Al}$ contrario, nella rivelazione il valore morale di un atto dipende dal suo contenuto oggettivo: ad esempio, il contenuto oggettivo di un atto di amore verso un nemico contiene una certa somiglianza con la perfezione di Dio (Wojtyła 1954 , p. 344). I due autori concordano invece sul fatto che le emozioni non collocano il soggetto in un mondo di cose, ma di oggetti di valore: Sachen, ovvero Ding und Wert, "cosa più valore” (Scheler 1913, pp. 42-45; Wojtyła 1954, p. 277).

\subsection{L'amore per la persona}

La realizzazione dei valori più elevati, religiosi, implica l'amore per le persone, compreso l'amor proprio. Persona è solo chi, nella gerarchia dei valori, è in grado di esperire i valori spirituali e del "sacro":

L'atto con cui cogliamo originariamente i valori del sacro è invece l'atto di un determinato tipo di amore (il cui orientamento assiologico precede e determina ogni rappresentazione ed ogni concetto dei dati del sacro): per sua essenza è orientato verso persone, cioè verso qualcosa di esistente in forma personale, a prescindere dal contenuto che vi possa inerire e dal "concetto" di persona che vi venga presupposto. Il valore irrelato nella sfera dei valori del "sacro" è pertanto, per sua essenza, un "valore personale". (Scheler 1913, p. 147)

In una formula: se l'ami, allora è una persona (incluso l'amor proprio). La persona è una forma di unità peculiare per questi valori. Per questo motivo non è necessariamente vivente: questa conseguenza permette il culto dei morti e dei santi. Scheler intende l'amore alla maniera degli autori antichi, ossia privo di aspirazioni di appagamento e dotato di un fine trascendente: manifestare apertamente i valori dell'oggetto. L'amore permette un contatto con la struttura 
Persona e comunità nel pensiero politico di Karol Wojtyła assiologica dell'oggetto amato a priori e non mediato dalla razionalizzazione:

Anche la componente emotiva dello spirito, la percezione affettiva, il preferire, l'amare, l'odiare ed il volere hanno un originario contenuto a priori, non mutuato dal "pensiero": l'etica deve porlo in evidenza in modo del tutto indipendente dalla logica. (Scheler 1913, p. 92)

In tal modo, è abolita la distinzione tra valori sperimentati e ideali. Tuttavia, l'amore non è un atto etico finché non si rivolge a una persona.

\subsection{La sequela}

Un tema di grande interesse per la semiotica delle religioni è costituito dall'analisi della sequela, ovvero della relazione che si dà tra un maestro, una guida morale, e un seguace. Si tratta di spunti interessanti anche in altri campi in cui reperiamo guide carismatiche, guru, follower e via discorrendo.

Come si è detto, per via del suo approccio realista, Scheler minimizza il ruolo della coscienza nell'etica: essa non è la fonte dei valori, ma si limita a capitalizzarli. L'amore per la propria persona è l'origine dell'ideale etico sulla base del quale ogni uomo viene giudicato; l'amore per un'altra persona è l'origine della sequela. Con questo termine Scheler intende il fatto che l'altra persona diviene per l'amante un modello:

In riferimento al suo contenuto il modello è una struttura assiologica articolata nella sua forma unitaria in conformità all'unità personale, una qualità di valore specifica sotto forma di persona, mentre in riferimento all'esemplarità del contenuto esso è l'unità di una istanza del dover essere fondata sul contenuto stesso. (Scheler 1913, p. 702)

L'amante co-sperimenta l'ideale mondo dei valori della persona-modello, innestandosi coi propri atti intenzionali in quel mondo e facendolo proprio. L'ideale del maestro diviene ideale dei discepoli. È possibile chiedersi se il valore non abbia sempre una dimensione normativa, nonostante la presa di distanza di Scheler dalle etiche della sanzione di cui ci occuperemo al paragrafo seguente. Tuttavia, il modello trae la propria forza dall'esemplarità e non dalla normatività (1913, p. 704); l'amante subisce passivamente l'attrazione del modello (1913, p. 702). Infine: 
Visibile nella concrezione fenomenica correlata all'atto d'amore, il modello attrae ed invita: "seguirlo" non significa quindi volere o agire in termini di obbedienza ad un autontico comando o ad una raccomandazione pedagogica o ad un invito ad imitare (o ad azioni analoghe e comunque parzialmente eteronome), bensì donarsi liberamente al contenuto assiologico personale del modello in quanto termine di discernimento autonomo. Ci conformiamo al modello in quanto persona [...]. (Scheler 1913, pp. 703-704

Ricordando che per Scheler la nozione di persona è strettamente individuale, se ne ricava che si segue sempre un individuo particolare e non un tipo generale.

Scheler (1913, p. 709) giunge a elaborare una tipologia di modelli che chiama «tipi puri di persone»: il santo, il genio, l'eroe, l'organizzatore, il bonne-vivant; inoltre, in una nota che sembra scritta da Foucault ${ }^{10}$, precisa che in tutti i movimenti in opposizione ai valori ufficiali (protestantesimo, controriforma, romanticismo, ecc.) si ritrova sempre la tendenza a creare contromodelli rispetto ai modelli prevalenti. Pertanto, nel romanticismo l'“anima bella" è il contromodello del cittadino del xvin secolo, valutato e detestato alla stregua di un filisteo. Ma i contromodelli generati dal risentimento dipendono pur sempre dai modelli esistenti, rispetto ai quali sono strutturalmente simili (Scheler 1913, p. 713, n. 270).

3.6 Assenza di una sanzione etica nella sequela

Come abbiamo visto, rispetto ai modelli standard dell'azione in semiotica, quello proposto da Scheler si caratterizza per l'assenza di quel che la semiotica generativa considererebbe un'istanza di destinazione. Di conseguenza, la sua etica rifiuta la sanzione (1913, pp. 443 - 456). Per verificare la tenuta di questa interpretazione, occorre chiedersi chi è "Dio" per Scheler. Egli non è una persona-modello ${ }^{11}$ : l'idea di Dio sorge dall'esperienza morale, e in particolare si connette all'infinitamente santo. Le etiche religiose, che Schopenhauer critica come etiche dell'imperativo, sono tali solo se riconducono bene e male al volere divino; l'imperativo

10. Il lettore perdonerà l'ironia sull'opinione poco lusinghiera di Foucault circa la fenomenologia.

11. In effetti, non molte persone si sforzano di essere come Dio. 
Persona e comunità nel pensiero politico di Karol Wojtyła svanisce se bene e male sono considerati, con Tommaso D'Aquino, come l'essenza di Dio, per cui l'etica è comportamento "in Dio" (Scheler 1913, p. 266). Alla luce di quest'idea il "comandamento dell'amore" (Matteo 22, 37-40; Marco 12, 29-31) non è un comandamento vero e proprio: la sanzione etica è in tutti i casi qualcosa che ha poco senso, perché la persona dovrebbe sperimentare la più profonda felicità emozionale nella stessa azione moralmente buona, e la più profonda disperazione nell'azione moralmente cattiva. L'etica di Scheler fonda sulla modalità del volere che esclude il dovere - egli nota come per Schopenhauer "dover volere" è un controsenso (Scheler 1913, p. 232). Non c'è morale se si pongono premi o punizioni per l'azione. Questo aspetto del pensiero di Scheler, tuttavia, non convince Wojtyła (1954, pp. 297-299): nell'etica cristiana l'ideale etico è anche un ideale enunciato da Gesù Cristo ("siate perfetti”); l'ideale di Scheler è il contenuto di un'esperienza emozionale-conoscitiva, e dunque non è necessariamente pratico, al contrario di quello cristiano (Wojtyła 1954, pp. 316-320).

4. Discussione: la sequela tra fenomenologia e semiotica La sequela è una nozione centrale della fenomenologia di Scheler, perché gli permette di rispondere al problema posto dalla relazione Alter/Ego in maniera originale. Non si tratta di chiedersi come un soggetto cognitivo-razionale, talvolta trascendentale, arrivi alla conclusione che il mondoper-lui è uguale al mondo-per-gli-altri, ma di comprendere come persone, provviste di un lato passionale, si considerino incomplete senza far esperienza del valore investito nell'Altro ${ }^{12}$. L'amore permette all'amante di co-sperimentare gli atti intenzionali della persona amata e la sua assiologia ideale di valori, che diviene il contenuto degli atti emozionaliconoscitivi dell'amante ${ }^{13}$.

L'assenza dichiarata della sanzione nel percorso che in Scheler lega passione e azione è piuttosto interessante. Ci

12. Non si sottovaluti l'originalità di questa posizione. È vero che l'Amore rappresenta per Scheler un grado superiore di simpatia, il che avvicina la soluzione a quelle che risolvono il rapporto Alter/Ego in una relazione di empatia - Husserl (1931), Stein (1917). Ad essere diversi sono i terminali della relazione, i valori in gioco e i modi di esperirli.

13. Usiamo "amante" e "amato" non per un vezzo post-strutturalista, ma per evitare la faticosa ripetizione del termine "persona". 
si può chiedere perché il maestro non sia il destinante del seguace. Una risposta, non priva di problemi, è che i valori positivi incarnati nel maestro determinano il sorgere immediato di valori nel discepolo: si tratta di una intuizione emozionale. Il rapporto tra maestro e discepolo non è determinato da un contratto o da una forma di manipolazione, come accade nel modello narrativo classico; è un rapporto di buon esempio. Il maestro non dà norme al discepolo: la norma è l'opposto dell'esperienza descritta da Scheler. Come si è detto, anche il "comandamento dell'amore" è, per Scheler, un non-comandamento. In effetti, impone di amare. A mio modesto modo di vedere si tratta di un esempio della paradossalità tipica del discorso mistico (cfr. Katz 1992). Vediamo il mondo in cui il comandamento è enunciato in Luca $(10,25-28)$ :

Un dottore della legge si alzò per metterlo alla prova: «Maestro, che devo fare per ereditare la vita eterna?». Gesù gli disse: «Che cosa sta scritto nella Legge? Che cosa vi leggi?». Costui rispose: «Amerai il Signore Dio tuo con tutto il tuo cuore, con tutta la tua anima, con tutta la tua forza e con tutta la tua mente e il prossimo tuo come te stesso». E Gesù: «Hai risposto bene; fa' questo e vivrai».

Nella versione di Luca, il maestro non dà alcun comandamento. Si limita a convenire col dottore della legge, la cui intenzione originaria era mettere Gesù alla prova. È vero che Gesù ha l'ultima parola, sanzionando positivamente ciò che dice il dottore e rovesciando il rapporto tra i due, ma questo avviene entro l'economia complessiva di un percorso che, con Eric Landowski (2006), potremmo considerare "di aggiustamento" tra due soggetti che finiscono per trovarsi in accordo reciproco. Ancora una volta, Gesù è un maestro paradossale, un maestro che non insegna.

\subsection{Assenza di gerarchia}

La nozione scheleriana di intuizione emozionale (dell'amato nella sua bontà morale) non è priva di problemi: perché il buon esempio, che implica l'osservazione e la condivisione empirica dell'esperienza, dovrebbe essere considerato alla stregua di un'intuizione? La semiotica narrativa può aiutare a inquadrare la situazione: le azioni morali del maestro (performance) portano a un esito positivo (sanzione) da parte del discepolo, che osserva, sperimenta e condivide i valori: 

nel pensiero politico di Karol Wojtyła è questa una condizione perché avvenga il trasferimento cognitivo. In sostanza è il discepolo a incarnare il ruolo di destinante. Più in generale, infatti, Landowski (2010) ha definito la deferenza come il manifestare rispetto davanti a quanto si giudica meritarlo.

In seguito alla sanzione positiva, tuttavia, il seguace si adatta al comportamento del maestro per aggiustamento, ovvero secondo una modalità di interazione che non è quella tra destinante e soggetto, bensì quella che si dà tra due soggetti sul medesimo livello gerarchico - cfr. Landowski (2006). Infatti, come si è visto, il vero maestro morale non ha bisogno di porsi a un livello gerarchico superiore, emettendo norme; il fatto di seguire il suo esempio è una libera decisione del discepolo, decisione motivata solo dal valore, rispetto al quale il maestro non è altro che un medium.

In maniera simile, Landowski (2014, mia traduzione) ha distinto due forme di rispetto verso l'Altro: la prima è mediata da norme sociali; nella seconda:

Laccordo consiste nell'accettare, preferibilmente in modo tacito, il principio del superamento o almeno della sospensione di queste condizioni, in altre parole assumendo i rischi di una relazione incondizionata ma anche allo stesso tempo, le possibilità di una interazione senza uno scopo deciso in anticipo, un fine "avventuroso".

La differenza tra le due modalità corrisponde grossomodo a quella che in francese e in italiano passa tra un rispetto "morale" (mediata da norme sociali) ed "etico" (che prescinde da tali norme $)^{14}$. Certamente, l'aggiustamento del seguace nei confronti del maestro non è sempre perfettamente simmetrico, come negli esempi di Landowski sui ballerini che "si aggiustano" tra loro; tuttavia, il fatto che la relazione tra maestro e seguace non sia simmetrica, ma complementare, non presuppone in alcun modo lo stabilirsi di gerarchie. Se il nostro punto di vista è corretto, all'intuizione di Scheler, che rimane piuttosto misteriosa, dovremmo piuttosto sostituire la semiosi, ovvero il rapporto tra manifestante (azione) e manifestato (valore). 
Vi sono poi ulteriori problemi. Scheler non sembra considerare la possibilità di un affrancamento del seguace dal maestro, che in realtà si riscontra piuttosto di frequente nei rapporti di sequela. Qui vorremmo sottolineare una differenza tra lo sguardo fenomenologico e semiotico. Scheler, come molti fenomenologi, tende a isolare, "per distillazione", dei tipi puri. Al contrario, la semiotica affronta testi in cui ci si imbatte sempre e soltanto in dati spurii. È bene essere consapevoli che la riduzione dei secondi ai primi, per quanto si renda talvolta necessaria per raggiungere una forma di sapere generale e utilizzabile, comporta tuttavia sempre un impoverimento. Come esempio, rispetto al problema della sequela del rapporto con il maestro, guarderei a Etty Hillesum, uno dei massimi esempi di riflessione mistica "laica" novecentesca. Intellettuale con una formazione linguistica, all'età di 27 anni la Hillesum entrò in analisi e intrecciò una relazione con il proprio terapeuta, Julius Spier, del quale divenne segretaria. Era il 1941, nel pieno dell'occupazione nazista dell'Olanda. Nel suo diario ci imbattiamo nella seguente osservazione:

La sorgente di ogni cosa ha da essere la vita stessa, mai un'altra persona. Molti invece - soprattutto donne - attingono le proprie forze da altri: è l'uomo la loro sorgente, non la vita. Mi sembra un atteggiamento quanto mai distorto e innaturale. (Hillesum 1986, p. 50)

Come testimonia il diario, il suo intraprendere gradualmente la via della mistica corrisponde a un rapido affrancamento dal rapporto di sequela con lo Spier, che pure l'aveva introdotta a letture spirituali - in particolare Agostino.

Gli esempi di affrancamento abbondano anche tra i consacrati: mi sono già occupato della relazione tra Padre Pio e i propri direttori spirituali in termini di agone, una sfida vera e propria che porta Padre Pio a rovesciare il rapporto di direzione (cfr. Ponzo, Galofaro 2019, pp. 123-146). Quindi, nella sequela non tutto "fila liscio" come lo descrive Scheler. Il comportamento del maestro è in un certo senso sempre sotto inchiesta da parte dell'allievo, che lo sfida costantemente a mantenersi all'altezza del proprio ruolo, sotto minaccia di perderlo (Greimas 1982). 
Persona e comunità nel pensiero politico di Karol Wojtyła
4.3 La sequela e la crisi della società occidentale

Lesplorazione delle radici della fenomenologia di Wojtyła nelle pagine dedicate all'etica di Max Scheler ci ha permesso di trovare una possibile risposta all'experimentum crucis che l'intersoggettività costituisce per le scienze umane. La sequela non riguarda soltanto il fenomeno religioso; piuttosto, si direbbe che molte altre relazioni, da quelle che si danno nei social network a quelle politiche ${ }^{15}$, dai fenomeni di contagio (Landowski 2004) - alla formazione di comunità sociali e politiche attive, esibiscano una struttura religiosa. In quanto fenomeno di co-sperimentazione passionale del valore, la sequela è costitutiva del legame sociale.

Sarebbe errato tuttavia credere che tali comunità si formino a partire dagli individui. Come si è detto, per Scheler, l'individuazione è un percorso faticoso di affrancamento dal collettivo; per Husserl, addirittura, l'intersoggettività è la condizione di possibilità dei soggetti. Che ci si ponga in un'ottica realista o trascendentalista, la conclusione è simile: le teorie che spiegano la società come una costruzione cercando un fondamento nell'esistenza dell'individuo postulano ciò che dovrebbero dimostrare: sono ideologiche.

Sarebbe un grave errore considerare la critica mossa da Wojtyła all'alienazione delle società comuniste del suo tempo come l'esito delle sue inclinazioni spirituali. Al contrario, come abbiamo visto, la sua indagine adotta i metodi e le prospettive della fenomenologia scientifica di stampo realista. Se il legame tra Wojtyła e Scheler che abbiamo ricostruito è corretto, è possibile affermare che la classe dirigente della Polonia comunista degli anni Settanta non era più in grado di stabilire una relazione basata sull'intuizione emozionale col proprio popolo, di co-sperimentare i valori dell'etica. La Chiesa cattolica è subentrata al Partito nel riempire questo vuoto di significato.

E lecito sospettare che la pandemia attuale abbia fatto emergere un problema identico: la crisi contemporanea

15. Secondo Landowski (comunicazione personale), la condivisione immediata, basata sull'intuizione emozionale, non riguarda solo la trasmissione di valori etici. Qualcosa della stessa natura sembra intervenire nell'adesione politica per contagio estesico. Un leader in grado di stabilire questo genere di relazione non è il destinatario dei suoi fanatici sostenitori, è il loro "buon esempio incarnato". La sua forma di presenza sensibile scatena immediatamente l'adesione, non la natura più o meno "persuasiva" dei suoi discorsi. Cfr. Landowski (2018). 
delle società liberali. In linea con l'analisi di Wojtyła, il gretto egoismo individuale che le fonda sembra renderle incapaci di promuovere un senso di appartenenza alla comunità umana a partire da un destino condiviso ${ }^{16}$. Le società comuniste superstiti avvertono il problema della ricerca di un fondamento morale: è il caso della riscoperta di grandi maestri, quali Confucio per la Cina, José Martí per Cuba. Nelle società occidentali, la funzione della sequela sembra essere oggi implementata, ben più modestamente, dai social media, senza che si intravveda una nuova visione filosoficomorale adeguata a compensarne il vuoto assoluto.

Bibliografia ${ }^{17}$

Ales Bello A., Pellegrino M.P. (a cura di)

2014 Edith Stein, Gerda Walther, Incontri possibili: empatia, telepatia, comunità, mistica, Castelvecchi, Roma.

Bertrand D.

2000 Précis de sémiotique littéraire, Nathan, Paris (trad. it. Basi di semiotica letteraria, a cura di G. Marrone, A. Perri, Meltemi, Roma, 2002).

Derrida J.

1987 Heidegger, l'enfer des philosophes, «Le Nouvel Observateur», 6-12 novembre, https://studylibfr.com/ doc/1591522/derrida-heidegger-enfer-des-philosophes.

Fantolino R.

2008 Il comunismo un male necessario? Perché Giovanni Paolo II non ha sconfitto il comunismo, Armando, Roma.

Faye E.

2005 Heidegger, l'introduction du nazisme dans la philosophie, Albin Michel, Paris (trad. it. Heidegger, l'introduzione del nazismo nella filosofia, L'Asino d'oro, Roma, 2012).

Galofaro F.

2021 On the Juridical Relevance of the Phenomenological Notion of Person in Max Scheler and Edith Stein, "International Journal for the Semiotics of Law - Revue internationale de Sémiotique juridique», online, https://doi. org/10.1007/s11196-021-09823-z.

16. L'espressione è cara a Xi Jinping (2017, p. 51). In effetti, a causa della misteriosa ironia della storia, la promozione di un senso di appartenenza alla comunità sembra riuscire meglio alle società comuniste sopravvissute, per motivi tutti da indagare.

17. Ove presente, il numero di pagina si riferisce all'edizione italiana. 

nel pensiero politico di Karol Wojtyła
Gorret D.

2019 Martin Heidegger e i Quaderni Neri: Pensiero, Politica, Poesia, https://www.doppiozero.com/materiali/martinheidegger-e-i-quaderni-neri.

Greimas A.

1982 Le défi, «Actes sémiotiques. Bulletin», n. 27 (poi in Greimas 1983), pp. 205-216.

1983 Du sens II. Essais sémiotiques, Seuil, Paris (trad. it. Del senso 2, Bompiani, Milano 1984).

Greimas A.J., Courtés J.

1979, Sémiotique: dictionnaire raisonné de la théorie du langage, Hachette, Paris (trad. it. Semiotica: Dizionario ragionato della teoria del linguaggio, a cura di P. Fabbri, Paravia Bruno Mondadori, Milano 2007).

Greimas A.J., Fontanille J.

1991 Sémiotique des passions: Des états de choses aux états d'âme, Seuil, Paris (trad. it. Semiotica delle passioni: dagli stati di cose agli stati d'animo, Bompiani, Milano 1996).

Heidegger $\mathrm{M}$.

1976 Essere e tempo [1927], Milano, Longanesi.

2018 Quaderni neri 1942-1948. Note I-V, Bompiani, Milano. Hillesum E.

1986 Het vertstoorde leven: dagboek van Etty Hillesum 19411943, Uitgeverij balans, Amsterdam (trad. it. Diario 1941-1943, Adelphi,, Milano 1985).

Hjelmslev L.

1943 Omkring spragteoriens grunglaeggelse, Akademisk forlag, Copenaghen (nuova ed. 1976; trad. ingl. Prolegomena to a theory of language, University of Wisconsin Press, Madison 1963; trad. it. dall'ingl. I fondamenti della teoria del linguaggio, Einaudi,, Torino 1968).

Husserl E.

1931 Méditations cartésiennes, Vrin, Paris (trad. it. Meditazioni cartesiane e Lezioni parigine, La Scuola, Brescia 2017).

1965 La filosofia nella crisi dell'umanità europea (conferenza di Vienna) [1935], in La crisi delle scienze europee e la fenomenologia trascendentale, il Saggiatore, Milano, pp. 309338.

Katz S.

1992 "Mystical Speech and Mystical Meaning", in Mysticism and Language, Oxford University Press, Oxford.

Landowski E.

2004 Passions sans nom, PUF, Paris.

2006 Les interactions risquées, «Actes sémiotiques», 101-103, 
(trad. it. Rischiare nelle interazioni, FrancoAngeli, Milano 2010).

2010 Arringa per l'impertinenza, in Ceriani G., Landoowski E., Impertinenze, et al., Milano, pp. 15-24.

2014 A quoi sert la construction de concepts?, «Actes sémiotiques», 117, https://www.unilim.fr/actes-semiotiques/5054.

2018 Populisme et esthésie, "Actes sémiotiques», 121, https:// www.unilim.fr/actes-semiotiques/6021.

Lévinas I.

1961 Totalité et infini, Martinus Nijhoff, La Haye (trad. it. Totalità e infinito. Saggio sull'esteriorità, Jaca Book, Milano 2016).

Losurdo D.

1991 La comunità, la morte, l'Occidente: Heidegger e l'ideologia della guerra, Bollati Boringhieri, Torino.

Maslow A.

1954 Motivation and Personality, Harper \& Brothers, New York.

Ott H.

1993 Heidegger: A Political Life, HarperCollins, London. PL

64 Manlii Severini Boetii Opera Omnia, in Patrologia Latina, a cura di J.P. Migne, vol. 64, 1847, pp. 1337-1354.

Ponzo J., Galofaro F. (a cura di)

2019 Semiotica e santità: prospettive interdisciplinari, CIRCe, Torino.

Reale G., Styczen T. (a cura di)

2003 Karol Wojtyta, metafisica della persona: tutte le opere filosofiche e saggi integrativi, Bompiani, Milano.

Scheler M.

1913 Der Formalismus in der Ethik und die materiale Wertethik, A. Francke, Bern (nuova ed. 1966; trad. it. Il formalismo nell'etica e l'etica materiale dei valori, San Paolo, Torino) 1996.

Simondon G.

2005 Lindividuation à la lumière des notions de formes et d'information, Jérôme Millon, Grenoble (trad. it. Lindividuazione alla luce delle nozioni di forma e di informazione, Mimesis, Milano 2011).

Spiegelberg H.

1971 The Phenomenological Movement: an historical introduction, 2 voll., Martinus Nijhoff, The Hague 
Stein E.

1917 Zum Problem der Einfühlung (dissertazione), Halle (trad. it. Il problema dell'empatia, Edizioni Studium, Roma 2014).

1999 Psicologia e scienze dello Spirito: contributi per una fondazione filosofica [1922], Città Nuova, Roma.

Szostek A. (a cura di)

1973 Discussion sur l'ouvrage du Cardinal Karol Wojtyta intitulé: „Osoba $i$ czyn” (La personne et son agir; Person und Tat), «Analecta Cracoviensia», 5, pp. 265-272 (https:// czasopisma.upjp2.edu.pl/analectacracoviensia/article/ view/2683).

Weil S.

1955 "Y-at-il une doctrine marxiste?", in Oppression et liberté, Gallimard, Paris, pp. 225-226.

Wojtyła K.

1954 Valutazioni sulla possibilità di costruire l'etica cristiana sulle basi del sistema di Max Scheler, in Reale, Styczen 2003, pp. 249-450.

2001 Persona e atto [1969], Bompiani, Milano (poi in Reale, Styczen 2003, pp. 829-1216).

2003 Saggi integrativi, in Reale, Styczen 2003, pp. 13031511 .

2005 Memoria e identità, BUR Rizzoli, Milano.

Xi Jinping

2019 The Governance of China. II, Foreign Language Press, Beijing (trad. it. Governare la Cina. II, Giunti, Firenze 2019). 


\section{L'Oggettuale e la Persona nella cultura digitale}

Gianmarco Giuliana

\section{Introduzione}

Lidea che il contesto tecnico e scientifico di un dato periodo storico ne influenzi anche il modo di pensare e di concepire il mondo non è nuova e nemmeno originale. In alcuni casi, infatti, è ben noto come anche interi movimenti culturali possano essere compresi soltanto alla luce di questi fattori: dalle forme del futurismo all'isotopia della scomposizione in Kandinsky. Molto è stato già detto anche sul tema specifico dell'impatto della macchina e del macchinario sulle società degli uomini, dal mutamento del lavoro alla scomparsa dell'aura nell'opera d'arte (Benjamin 1936). Tuttavia, laddove molte discipline umanistiche ci hanno insegnato e continuano a insegnarci l'importanza di guardare al passato per comprendere il nostro presente, la specificità dello sguardo semiotico risiede invece in quella audacia, caratteristica di opere come Il neobarocco (Calabrese 1987) o i Miti d'oggi (Barthes 1957), di guardare alla propria contemporaneità tendandone una interpretazione sincronica che tenga conto delle forme dominanti della Storia. È dunque in questa ottica che si lavorerà in questo articolo, partendo da una semplice osservazione: lo sviluppo tecnologico degli ultimi sessant'anni negli ambiti della robotica e dell'informatica ha sin da subito e ininterrottamente posto il problema della 
definizione della persona e della sua possibile estensione categoriale a un oggettuale dotato di intelligenza, agency e linguaggio. Osservazione che ci porta a delineare quello che sarà il nostro oggetto di indagine e la nostra domanda di ricerca: come si articola nei videogiochi, figli di quello sviluppo passato e in parte padri di quello attuale, l'opposizione fra oggetto e persona? Come infatti affermano Bonneuil e Pestre (2015, p. 9, mia traduzione:

Il secolo che comincia con la Prima Guerra mondiale è quello della penetrazione dei saperi scientifici e dei loro immaginari in tutti gli aspetti della vita sociale, economica e culturale.

Si tratta di un secolo che gli studiosi definiscono come quello delle tecnoscienze le cui pratiche assumono una fortissima dimensione discorsiva (ivi, p. 18). Indagare i videogiochi, al tempo stesso prodotti di quel discorso scientifico e produttori di una discorsività del computazionale, diviene allora fondamentale. Certo è che essi non possono essere considerati come i primi prodotti culturali ad aver affrontato la questione, e che questa indagine potrebbe venire svolta su altri fronti. Pensiamo infatti a come già nel 1927 Fritz Lang ponga la figura dell'androide al centro di Metropolis, e a come ancora cento anni prima, con Frankenstein; or, the Modern Prometheus (1818), Mary Shelley tematizzi anche il problema di una persona-oggetto. Peraltro anche dal punto di vista della contemporaneità non si può non riconoscere la centralità del cinema con quei film cult che hanno al loro centro proprio questa tematica: 2001: A Space Odyssey (Kubrick 1968); Westworld (Crichton 1973); Blade Runner (Scott 1982); RoboCop (Verhoeven 1987); Bicentennial Man (Columbus 2000); e anche i più recenti Ex Machina (Garland 2015) e Her (Jonze 2013).

Un discorso, quello cinematografico, che peraltro si potrebbe estendere anche alle serie televisive con il caso emblematico di Star Trek: The Next Generation (Roddenberry 1987) con il personaggio centrale di "Data" e intere puntate dedicate proprio al tema dell'androide ("The Measure of a Man”, 1989), ma anche di Knight Rider (Larson 1982 - noto come Supercar in Italia) con i dialoghi ${ }^{1}$ fra l'intelligenza della

1. https://www.youtube.com/watch?v=HK2QymEvO_c. 
macchina KITT e il protagonista umano che spesso affida alla "macchina" molti compiti. Tuttavia se le opposizioni semantiche fra persona e oggetto non sono certo una novità, e possono addirittura essere ritrovate nella mitologia greca con la "statua vivente" Talos e più in generale nelle riflessioni di autori greci e latini sull'automa e sulla macchina (Choppin, Collognat 2020), diventa interessante indagare il modo in cui questa opposizione cambi nelle diverse narrazioni. Per esempio, mettendo a confronto Metropolis con I, Robot (Proyas 2004) è facile vedere come in meno di cento anni la posizione attanziale della macchina dalle sembianze umane e i valori assiologici inseriti su un elemento figurativo come la mano protesica siano diametralmente opposti. Similmente, la drammaticità del finale dell'episodio "The Lonely" della serie Twilight Zone (Serling 1956), episodio in cui un uomo giunge a preferire la solitudine su un asteroide insieme a un androide donna di cui si è innamorato piuttosto che il ritorno sulla terra, è lungi dall'essere ovvia o scontata nel momento in cui assumiamo una prospettiva storicoculturale. Far luce su queste trasformazioni è dunque ciò che proveremo a fare in questo contributo, guardando ai videogiochi come a una forma di testualità che in virtù della specifica attività interpretativa che richiede dispiega questa antica opposizione semantica in una maniera inedita e di grande interesse per le scienze umanistiche.

2. Quattro buoni motivi per cui indagare il concetto di persona attraverso $i$ videogiochi

Mettendo in rapporto il cambiamento tecnologico e la concettualizzazione della persona, i giochi digitali diventano un oggetto di studio da privilegiare per almeno quattro motivi. Il primo riguarda una certa consapevolezza sociale secondo cui i videogiochi non sono una tecnologia fra altre, bensì un modello insito in molte tecnologie del digitale. Scrive per esempio Baricco nel suo recente The Game:

La dico in modo brutale: per ragioni storiche e per così dire darwiniane, da un certo punto in poi (dall'IPhone in poi se dovessi azzardare una data) nulla ha più avuto serie possibilità di sopravvivenza se non aveva nel DNA il patrimonio genetico dei videogame. (Baricco 2019, p. 150) 
Un modello che dunque non può non avere un ruolo in quel cambiamento per cui

[i] nostri gesti già sono cambiati, con una velocità sconcertante, ma i pensieri sembrano essere rimasti indietro nel compito di nominare quello che creiamo a ogni istante. Non sono già più uguali lo spazio e il tempo, da un po': sta succedendo la stessa cosa a luoghi mentali che per lungo tempo abbiamo chiamato passato, anima, esperienza, individuo, libertà. (ivi, p. 15)

Il secondo motivo riguarda il funzionamento stesso dei videogiochi, costituiti (anche qui come molte altre tecnologie) da invisibili processi informatici sottostanti e caratterizzati proprio dal simulare intelligenze artificiali che sovente sono antagoniste rispetto alla persona del giocatore ma che al tempo stesso lo guidano nelle sue scelte e aiutano in diversi modi. Proprio questi due tratti sono stati molto recentemente associati a una possibile fine dell'individuo dal filosofo Gaspard Koenig (2019, p. 21, mia traduzione):

Se un algoritmo mi conosce meglio di me stesso e mi propone delle scelte più razionali di quelle che avrei fatto, se una miriade di oggetti interconnessi anticipano la mia capacità di decisione offrendomi una esistenza determinata e confortevole, se smetto a poco a poco di essere l'agente delle mie stesse azioni, perché effettivamente dovrei avere diritto di voto ed essere sottomesso alla minima responsabilità giuridica? L'IA sferrerebbe il colpo di grazia al libero arbitrio, e con lui all'idea kantiana dell'autonomia del soggetto.

Il terzo motivo riguarda il luogo in cui essi sono divenuti un fenomeno di massa: il Giappone, luogo in cui lo sviluppo di tecnologie informatiche è stato al tempo stesso un fatto di salvezza economica e identitario (Tavassi 2012, $p$. 105) $e$ che è divenuto la patria per antonomasia dei videogiochi a discapito dell'origine occidentale del medium così come di alcuni dei suoi prodotti più iconici. Ovviamente non possiamo qui dilungarci sull'ampia letteratura riguardo questo argomento, ma possiamo e dobbiamo quanto meno notare come sia proprio quello il luogo in cui nasce una delle prime figure di persona tecnologicamente oggettificata e positivamente assiologizzata: Astro Boy (1952) di Ozamu Tezuka. 
Personaggio, questo piccolo ragazzino con un grande cuore elettronico, che può essere ricollegato non solo ad altri anime come Doraemon (Fujio 1970) ma persino a un intero genere testuale che è esclusiva dell'isola del sol levante, ossia quello dei mecha: da Mazinger $Z$ (Nagai 1972) a Evangelion (Anno 1995) in cui viene rappresentato un robot che sanguina dotato di una mente e di un'anima. Un genere che ha peraltro aperto a molte tematiche correlate, come nel notissimo caso di Ghost in the Shell (Shirow 1989) e nel forse meno noto caso di Serial Experiments Lain (Ueda, ABe 1998). Infine, si tratta di un genere che non ha mancato di influenzare intersemioticamente anche le narrative occidentali come nel caso di The Iron Giant (Bird 1999) e forse persino quel momento significativo nei comics in cui l'armatura di Iron Man (Lee 1963) diventa senziente e il protagonista ottiene un cuore artificiale ${ }^{2}$. È d'altronde significativo che il Giappone abbia anche il suo proprio Metropolis animato (Rintaro 2001) che presenta interessanti differenze nel senso da noi indicato. Esaminare a fondo queste serie (Lorusso 2010) di testi e queste tendenze storiche è compito di una semiotica della cultura che supera gli scopi di questo articolo, ma certo è che per un semiologo tutto questo difficilmente può essere visto come pura coincidenza. L'ultimo motivo, infine, riguarda l'interesse caratteristico della semiotica per la cultura popolare e per i suoi prodotti. Basti pensare qui a come Umberto Eco si sia dedicato alle poetiche dell'arte contemporanea (Eco 1962) ma anche a Superman (Eco 1964) e Tarzan (Eco 1978). Questi oggetti infatti contengono in sé una certa modellizzazione e concettualizzazione valorizzata del mondo, essi sono traccia di un ovvio e ci aiutano dunque a ricostruire una data configurazione enciclopedica entro cui questi oggetti hanno senso (Paolucci 2017, p. 208).

\section{Quale nozione di persona?}

Prima di indagare la nozione di persona nei videogiochi, bisogna tuttavia decidere prima a cosa ci riferiamo con questo termine. In modo consono alla metodologia semiotica, guardiamo dunque a una definizione dizionariale ${ }^{3}$ di

2. Iron Man, vol. 3, n. 26, marzo 2000.

3. http://www.treccani.it/vocabolario/persona/. 
questo concetto e selezioniamo le parti che ci sembrano di maggiore interesse:

1. a. Individuo della specie umana, senza distinzione di sesso, età, condizione sociale e sim., considerato sia come elemento a sé stante, sia come facente parte di un gruppo o di una collettività [...].

2. letter. a. Con il sign. etimologico, maschera teatrale, e quindi anche la parte che un attore rappresenta sulla scena. b. estens. La parte che si sostiene nella vita sociale; ufficio, funzione ufficiale, figura giuridica $[\ldots]$.

3. a. Il corpo, il fisico umano [...].

4. Nel linguaggio giur., ogni soggetto di diritto, titolare di diritti e obblighi, investito all'uopo della necessaria capacità giuridica $[\ldots]$.

Notiamo già qui qualcosa di molto interessante, e cioè che "persona" coincide innanzitutto con "umano" sia dal punto di vista corporeo-genetico che dal punto di vista politico (e dunque sociale). E in effetti proprio in questi termini che usiamo tipicamente questa parola e ci viene molto intuitivo opporre la persona e l'oggetto. Peraltro, la seconda definizione della persona come maschera coincide anche con la sua nozione linguistica in cui essa assume funzione deittica e, appunto, relazionale ${ }^{4}$. A completare questa nozione vi è poi l'accezione filosofica secondo cui:

5. Nel linguaggio filos., l'individuo umano in quanto è ed esiste, ossia intende e vuole, esperimenta e crea, desidera e ama, gioisce e soffre, e attraverso l'autocoscienza e la realizzazione di sé costituisce una manifestazione singolare di quanto può considerarsi essenza dell'uomo, nella sua globalità intellettiva e creativa, e come soggetto cosciente di attività variamente specificate (razionale, etica, ecc.): la dignità, il valore, la libertà, la creatività della $p$. umana $[. .$.$] .$

Questa nuova definizione è estremamente interessante perché introduce implicitamente alla dimensione ideologica della persona, a come cioè questo termine sia spesso sinonimo di una valorizzazione positiva dell'insieme di "umano" e "individuo". Ed è infatti sempre in ambito filosofico che possiamo vedere una riflessione critica sull'irriducibilità della persona all'umano e all'individuo. 

Individuo e Persona. Tre saggi su chi siamo (De Anna et al. 2007) e anche del dibattito bioetico sulla nozione di persona:

Se la persona è un composto di anima e corpo non possiamo partire presupponendo sin dall'inizio che l'anima sia presente dalla fecondazione nel corpo cioè nell'essere umano. [...] Non possiamo cioè dire che l'essere umano è sin dall'inizio persona perché altrimenti non avremmo argomenti contro l'aborto. [...] Presupporre che uguaglianza esiga la coincidenza di persona ed essere umano è, ancora una volta, dare per scontata la conclusione da dimostrare e in discussione. (Mori 2013, p. 164)

Già abbiamo visto che la persona è unione di corpo e anima, termine qui accolto per indicare le «funzioni superiori». Possiamo ora precisare che il corpo deve essere individuale: anche per questo il genotipo è in sé poco rilevante. Perché ci sia una persona sono necessarie due condizioni: l'individualità somatica e la razionalità («le facoltà superiori»). (ivi, p. 198)

Ovviamente non ci è possibile affrontare qui il problema di come la nozione di persona sia stata trattata nella storia della filosofia, e non si deve dunque prendere l'estratto da noi scelto come rappresentativo di una posizione comune. Parimenti non ci è possibile confrontare le definizioni di questo termine all'interno della molteplicità delle lingue e dei loro diversi dizionari, un lavoro sicuramente importante che tuttavia avrebbe richiesto un articolo apposito ${ }^{5}$. Quello che però è importante per la nostra riflessione è riuscire a isolare, attraverso queste diverse definizioni, un insieme di tratti generali che possano definire la persona a prescindere dalla sua natura umana. Diremo allora che la persona è:

- singola (individuabile);

- corporea/tangibile;

- capace (sente, comprende, vuole, agisce);

- relazionata (contestualizzata);

- valorizzata (l'attribuzione della qualità di persona avviene dall'esterno e non è neutra).

5. Tale compito è stato intrapreso in questo volume da Ugo Volli, che indaga la storia della nozione e del termine persona nel corso del tempo e riferendosi molto spesso alla tradizione filosofica. 
Come sarà evidente al nostro lettore, di queste cinque caratteristiche è sicuramente la terza quella che pone maggiore problema (non che le altre siano del tutto pacifiche). Certo è che le strategie comunicative attraverso cui personificare l'oggettuale toccano tutti e cinque i punti: in questo stesso istante un programma sul mio computer mi ricorda che è tempo di fare un "health check" al mio PC e che è bene farlo spesso così che esso rimanga "in good shape". Non di meno l'attribuzione metaforica di un corpo a un computer rimane meno problematica di una sua attribuzione agentiva, intellettiva e volitiva. Non a caso nell'introduzione di Intelligenza. Per una unione di intelligenza umana e artificiale (Ienca 2019) si sottolinea proprio la problematicità di definire l'intelligenza che, in virtù dell'importanza che le attribuiamo, è un concetto in qualche modo compromesso in partenza. Tuttavia questa terza caratteristica è anche semioticamente la più interessante proprio in quanto sarà essa che dovrà essere maggiormente finta in modo che possa venire attribuita la qualità di persona a ciò che pertiene al dominio dell'oggettuale. Non è un caso che Gaspard Koenig (2019, p. 64), che racconta di aver rimproverato i suoi figli per essere stati educati e gentili con Alexa ${ }^{6}$, concentri proprio su questa capacità di finzione la sua critica in quanto essa è anche il presupposto per assecondare poi quella tendenza animista che, rifacendosi a Bachelard, lo studioso definisce un ostacolo allo spirito scientifico:

Intraprendere una relazione affettiva con un robot non rappresenta l'avanguardia del progresso, ma una sua regressione per la nostra civiltà. Basta visitare il museo delle Arti Prime a Parigi per constatare come le società primitive si ingegnavano per attribuire un'anima, un potere e dei sentimenti a degli oggetti inanimati (il famoso mana dei Polinesiani). [...] Da un punto di vista puramente scientifico l'IA non pensa, non soffre, non ama. (Koenig 2019, pp. 62-63, mia traduzione)

Trovandoci tuttavia dal lato della semiotica e non da quello della filosofia (che è e rimarrà la nostra stella guida e compagna di viaggio), a noi tocca invece studiare proprio

6. Alexa è l'assistente vocale di Amazon basato su cloud ed è l'intelligenza che alimenta Amazon Echo e altri dispositivi. Interprentando il linguaggio naturale, può interagire con l'uomo e aiutarlo nello svolgimento di diversi compiti (come ordinare cibo a domicilio o informarsi sulle previsioni metereologiche). 
quali siano le strategie attraverso cui la nostra società e i suoi testi personifichino l'oggettuale. Infatti, se di solito è ovvio pensare che le persone abbiano una personalità che le contraddistingue (individualizza), da un punto di vista prettamente semiotico (per cui il soggetto è esso stesso segno e l'accesso al mondo è sempre mediato dal discorso) è vero esattamente il contrario: è perché ci è possibile riconoscere una personalità che crediamo che qualcosa sia una persona. In altre parole noi non abbiamo accesso alla persona in quanto tale, alla sua ontologia, ma solo alla sua discorsività e a una narrativizzazione da noi attribuita che in quanto tale può benissimo essere finta o erroneamente assegnata. Da questo punto di vista bisognerà allora indagare contemporaneamente due aspetti di questo processo. Il primo è quello secondo cui la persona viene definita ${ }^{7}$ concettualmente in opposizione all'oggetto:

2. Per estens. a. La cosa (materiale o non materiale) o la persona cui è diretta un'azione, un'attività, un comportamento, $\mathrm{o}$ alla quale è rivolto un sentimento. [...]

3 . In senso più concr. e più com., ogni cosa che cada sotto i sensi dell'uomo: la luce permette di distinguere i varî o.; non conosco l'uso di questo o.; in partic. (spec. con una determinazione), cosa che abbia una forma definita e sia opera del lavoro umano. [...]

6. In grammatica, s'intende per oggetto dell'azione verbale la persona o la cosa, concreta o astratta, su cui si esercita l'azione esplicata da un'entità agente, soggetto dell'azione, e che, nella frase con verbo transitivo attivo, costituisce il complemento diretto del verbo, cioè il complemento oggetto; questo, nelle lingue indoeuropee con declinazione, è indicato dal caso accusativo, nelle altre per mezzo della collocazione, in genere dopo il verbo. [...]

Qualunque strategia semiotica dovrà dunque in qualche modo neutralizzare, se non proprio capovolgere, queste proprietà passive dell'oggetto.

Ma è forse ancora più interessante il secondo aspetto per cui una strategia semiotica di personificazione dell'oggettuale potrà anche far partecipare (Paolucci 2010) e rendere compresenti le qualità dell'uno e dell'altro. D'altronde se pensiamo a una delle icone del mondo videoludico, a Mega-

7. http://www.treccani.it/vocabolario/oggetto/ (corsivo mio). 
man (Capcom 1987) e al suo cane Rush (1990) ci rendiamo conto che sia dal punto di vista figurativo che narrativo essi sono dei robot contraddistinti proprio dalla partecipazione dei tratti "organico" e "non-organico" e di una forma di intelligenza naturale e artificiale. Vedremo così che quello che contraddistingue concettualmente la nostra contemporaneità non è affatto solo un rovesciamento di una opposizione semantica (secondo cui, come teme Koenig, al mutare della posizione della tecnologia muta inevitabilmente anche la nostra rendendoci non più soggetti ma oggetti) ma bensì una nuova logica di partecipazione che segna il modo in cui è cambiata la nostra cultura e che contraddistingue non solo i personaggi delle storie dei videogiochi ma anche la tecnologia videoludica stessa. Alla luce di tutto questo, la nostra indagine può dunque iniziare.

\section{Processo semiotico di creazione dell'Oggetto-Persona}

Cominciamo col guardare al modo in cui i personaggi delle narrazioni videoludiche sono spesso oggetti che vengono personificati. Più precisamente, guarderemo alle quattro seguenti strategie:

- Loggetto come persona nelle narrazioni videoludiche come posizione attanziale: la macchina Aiutante \& Soggetto.

- L'oggetto come persona nelle narrazioni videoludiche come attore collettivo: la macchina parte indistinta del gruppo, la macchina come categoria naturale fra altre.

- Loggetto come persona nelle narrazioni videoludiche come figura: la macchina con braccia e gambe, la macchina col volto, la macchina con l'occhio.

- L'oggetto come persona nelle interazioni videoludiche come capacità: la macchina che ragiona attraverso il linguaggio.

4.1 I nuovi attanti delle narrative videoludiche: androidi, robot e intelligenze artificiali

Per quanto riguarda il primo punto, gli esempi di aiutanti e coprotagonisti robotici sono davvero numerosi: dai recentissimi Star Wars: Jedi of the Fallen Order (Respawn Entertainment 2019) e Star Wars Battlefront II (EA DICE 2017) fino ad andare indietro al 2004 con "Dog" in Half Life 2 (Valve), 

già citato Megaman. Persino la parte macchinica pilotata dal protagonista di Titan Fall (Respawn Entertainment 2014) è in realtà caratterizzata da una intelligenza artificiale con una personalità parziale e mostra di fatto di essere capace di una intenzionalità. Mentre invece in un caso come quello di ReCore (Comcept \& Atmature Studio 2016) i robot dalle sembianze animali che accompagnano la protagonista mancano di una personalità ma sono di fatto ciò grazie a cui essa può agire sul mondo. Infine merita di essere citato anche il "BB" di Death Stranding (Kojima Production 2019), al tempo stesso una tecnologia e un feto umano che accompagna e aiuta il personaggio principale. Alcuni di questi coprotagonisti meritano tuttavia una maggiore attenzione, come Wheatley in Portal 2 (Valve 2011), Cortana nella serie di Halo fino al quarto capitolo (Bungie Studio 2001-2012) e i "pod" (piccoli robot fluttuanti che seguono i protagonisti) di Nier Automata (Platinum Games 2017).

Per quanto riguarda il primo, esso viene definito come «The Intelligence Dampening Sphere, more commonly known as Wheatley, is a personality core of masculine programming and English West Country accent ${ }^{8}$. Wheatley assume un grande rilievo per la nostra indagine non solo perché ha un ruolo centrale nella trama in cui subisce un vero e proprio mutamento, ma anche perché appare come dotato di tutta una serie di tratti caratteristici della persona (fino appunto ad avere non solo capacità linguistica ma persino un particolare accento) in un contesto in cui l'avatar del giocatore ne è invece sprovvisto (non parla, non sappiamo nulla di chi sia né di cosa voglia, non ne vediamo quasi mai il volto, ecc.) e in cui questa mancanza viene messa in luce dal gioco stesso ${ }^{9}$. Il secondo caso, quello di Cortana, è invece importante per un fattore per così dire sociologico. Dal punto di vista narrativo Cortana viene definita ${ }^{10} \mathrm{come}$ «una IA dell'UNSC, il cui numero di serie è CTN-452/9» che assume tuttavia il suo tratto umano in quanto la «sua matrice fu creata dai tracciati celebrali della dottoressa Halsey: essa fu

\footnotetext{
8. https://theportalwiki.com/wiki/Wheatley.

9. Su questa interessante situazione di ribaltamento non ci dilunghiamo ma rimandiamo all' analisi di Fanny Barnabé (2017) che è dedicata proprio a questo tema.

10. https://halo.fandom.com/it/wiki/Cortana.
} 
creata con gli algoritmi di decrittazione e di intercettazione più avanzati e scelse di essere l'accompagnatrice del Capo». Da cui il fatto che essa sia descritta come «seria e diligente nello svolgere i suoi compiti, ma ha un'indole allegra e la battuta facile» e che abbia anche una particolare figurativizzazione dall'aspetto «di una bella ragazza "nuda" con pelle e capelli viola e il corpo solcato da circuiti elettrici».

Vediamo già qui molteplici delle strategie prima delineate, ma il vero motivo per cui la mettiamo in rilievo risiede nel fatto che alcuni commenti ${ }^{11}$, video di reactions ${ }^{12}$, fan $\operatorname{art}^{13} \mathrm{e}$ diversi meme ${ }^{14}$ siano nati in seguito a un evento narrativo in Halo 4 (Bungie 2012) in cui essa muore sacrificandosi per salvare il protagonista (e giocatore). La morte dell'IA sembra aver insomma molto colpito e commosso la comunità dei giocatori di uno dei generi videoludici forse più criticati: lo sparatutto (genere in cui il compito principale del giocatore è quello di uccidere senza sosta centinaia di nemici manipolando diversi tipi di armi da fuoco). Il terzo e ultimo caso dei pod in Nier Automata è invece importante in quanto essi sono coprotagonisti robotici di soggetti principali androidi dalle sembianze umane. Tutta la storia di Nier riguarda infatti il tema di ciò che diviene la cultura umana, dalle sue nozioni filosofiche alle sue pratiche, una volta che essa viene ereditata da creazioni oggettuali in un mondo privo di umani in cui delle creazioni a loro immagine e somiglianza (appunto gli androidi) sono in costante guerra contro dei robot extraterrestri che, una volta invasa la Terra, si sono nutriti della cultura umana e sono mutati in funzione di essa. Entrare nei dettagli della sua complessa trama ci è qui impossibile, ma basti dire che il giocatore si ritroverà davanti a tutta una serie di scene come una grande orgia di robot intenti (in vano) a riprodursi ${ }^{15}$ o ancora a dei "bambini robot" morti per suicidio dopo aver fatto evolvere la nozione di paura in

11. "Woman cried to Titanic, Men cried to Terminator 2, Gamers cried to Halo 4": https://www.youtube.com/watch?v=ACvIxGpzGs8.

12. https://www.youtube.com/watch? $v=u 4 C n 30$ Rqwdc.

13. https://www.reddit.com/r/fanart/comments/95fjbe/death_of_cortana_by_legion $5551 /$.

14. https://ifunny.co/picture/dad-why-the-fuck-you-crying-so-damn-loud-halovoJqCSXU5 ; https://memegenerator.net/instance/62287790/dragon-ball-z-angry-whencortana-dies-in-halo- 4 .

15. https://www.youtube.com/watch?v=h74TG_Ge8KI. 
angoscia e disperazione. Ebbene, all'inizio di tutto questo i protagonisti sono accompagnati e aiutati da un piccolo pod volante che non ha sembianze antropomorfe e che è dotato di limitatissime capacità linguistiche; pod che tuttavia svilupperà una coscienza e una personalità proprio in virtù delle comunicazioni e relazioni con le altre intelligenze non umane, al punto da determinare il true ending (vero finale "felice" fra molti altri possibili) stesso della storia.

4.2 Loggettuale fra partecipazione e inclusione

Ma veniamo ora alla seconda strategia, quella dell'inclusione ed estensione della categoria oggettuale. Essa avviene principalmente in due modi. Il primo è direttamente collegato alla posizione attanziale, e consiste proprio nell'inserire la figura del robot (nel suo termine esteso) all'interno di gruppi (semioticamente unità) di personaggi, legando cioè queste figure oggettuali a una appartenenza comunitaria oltre che a un preciso lato dello schema attanziale: essi sono non più solo coprotagonisti ma alcuni fra/come altri. Anche qui gli esempi sono numerosi, dall'NPC robot reclutabile "Vax" in Wasteland (Interplay Entertainement 1988), a Robo in Chrono Trigger (Squaresoft 1995) e fino a agli emblematici Legion di Mass-Effect 2 (BioWare 2010) e HK-47 di Star Wars KOTOR (BioWare 2003), entrambi contraddistinti dal loro non essere umani sia per un caratteristico cinismo che per una imperfetta capacità linguistica. Ma troviamo questa situazione anche in giochi molto meno narrativi, come Pathfinder in Apex Legends (Respawn Entertainment 2019) o ancora in League of Legends (Riot Games 2009) in cui troviamo sia personaggi robotici che skin (rappresentazioni figurative alternative rispetto a quelle originali) robotiche per personaggi umani e creature di vario tipo. Da notare infine qui due casi recenti: quello di Fallout 4 (Bethesda 2015) e quello di Persona 5 (Atlus 2016). Nel penultimo capitolo di Fallout (2015) troviamo ben quattro personaggi robotici reclutabili, contro uno solo nel terzo capitolo del 2008. In Persona invece possiamo vedere come, se nei capitoli precedenti vi era già stata una protagonista androide nominata "Aigis" (Persona 3, Atlus 2006), l'ultimo titolo ci presenti un personaggio (Futaba) femminile il cui "spirito" ha un nome che si riferisce a un tomo "mitologico" di Lovecraft (Necronomicon) ma che figurativamente è rappresentato come un UFo pieno 
di "computer" con cui lei interagisce e che lei pilota come veicolo. La dimensione oggettuale ricopre in questo caso dunque anche una figura spirituale, mitica e psicologica.

Il secondo modo in cui questa inclusione avviene è però forse ancora più significativo, e può essere osservato in uno dei titoli più noti e giocati al mondo: Pokémon. Sin dalla prima generazione (Game Freak 1996) è evidente come la maggior parte dei pokémon siano esseri estremamente simili a degli animali esistenti e legati ad alcuni elementi naturali, eppure vi sono tre evidenti eccezioni: Magnemite (un simil magnete), Porygon (una figura tridimensionale) e Voltorb (una sfera elettrica dall'aspetto di una pokeball). Non soltanto essi appartengono a una dimensione oggettuale, ma il loro far parte di uno stesso gruppo non viene inizialmente problematizzato e bisognerà aspettare la seconda generazione per vedere esplicitato nel pokedex (piccolo computer portatile che contiene molti dati) questa appartenenza insolita. E proprio nella seconda generazione possiamo vedere come i pokémon oggettuali si riconfermino come una presenza caratteristica della serie e assistiamo addirittura alla nascita di una nuova tipologia di pokémon: acciaio. La scelta dell'acciaio come tipologia insieme alle altre, che appartengono o agli elementi naturali (fuoco, acqua, ecc.) o a una dimensione antropologico-simbolica del vivente (psico, fantasma, folletto, ecc.), è significativa dato che si tratta di un materiale che è caratterizzato da un intervento umano. Questa tendenza continuerà peraltro nel tempo, per cui avremo pokémon portachiavi, ingranaggi, spade, e così via fino al caso della forma perfetta di Zygarde, pokémon narrativamente legato alla mitologia e fatto di cellule ma che dal punto di vista figurativo è rappresentato secondo i tratti tipici del mecha. Non sorprenderà dunque che nel capitolo "Luna e Sole" (2016) il pokedex stesso del protagonista venga integrato come un pokémon, Rotom, e assume dunque un volto e una personalità. Questa tendenza peraltro non riguarda solo la serie Pokémon, ma può essere riscontrata anche nella serie dei Final Fantasy in cui se in passato alcune fra le "semi-divinità" di quei mondi magici avevano una natura eccezionalmente oggettuale (il caso più eclatante essendo Ark in Final Fantasy IX del 2000, un vero e proprio dio-mecha in un universo fantasy per nulla futuristico) col tempo, nel tredicesimo capitolo (2009), il tratto meccanico-oggettuale è 
diventato assolutamente dominante. Così il semidio-cavaliere Odino diventa un robot meccanico che può trasformarsi in un cavallo riorganizzando le sue parti e il drago Bahamut allo stesso modo diventa un aereo da caccia. E, a dimostrazione della compartecipazione semantica dell'oggettuale con ciò che gli è tradizionalmente opposto, entrambi sono creati da una dea che ha rinchiuso il loro spirito in un cristallo magico. Infine, se da un lato assistiamo dunque a un oggettuale sempre più "vivo" e personificato, dall'altro in un gioco come Horizon Zero Dawn (Guerilla Games 2017) vediamo come l'intero mondo naturale viene immaginato come popolato da selvaggi animali meccanici che la protagonista cacciatrice dovrà domare e combattere grazie a lance e fionde tecnologiche. Una chiara dimostrazione del dialogo culturale in corso e della natura relazionale del significato e dei valori investiti su questi termini.

Tutti questi esempi ci mostrano dunque come la dimensione oggettuale diventi una categoria che partecipa non solo a quella del vivente ma ricopre a volte addirittura posizioni metafisiche e trascendentali; in più occasioni l'oggetto e il divino infatti coincidono. A riguardo, non si può non citare la serie Xenoblade Chronicles (Monolith Soft 2010) in cui l'oggettuale meccanico assume il ruolo di una semidivinità all'origine del mondo e in eterno conflitto con il mondo dell'organico (e dunque non più come un oggetto creato $d a$ ):

Long ago, the world was nothing more than an endless sea cloaked in a boundless sky, reaching as far as could possibly be imagined. [the clouds part to reveal two giants - one white, the other black] Then two great titans came into existence: the Bionis and the Mechonis. ${ }^{16}$

La scelta semantica del "meccanico" è estremamente interessante in quanto rimanda non solo a ciò che è «di macchina, che riguarda le macchine o in qualsiasi modo ha rapporto con esse» ${ }^{17}$ ma più in particolare all'idea di un agire "che si fa o si compie per mezzo di macchine o di meccanismi varî: aratura, trebbiatura m.; trazione m. (contrapposta alla trazione animale); lana $\mathrm{m}$., lana rigenerata. e. Per estens., di ogni operazione che si compie quasi automaticamente, sen-

16. Dialogo tratto dalla cutscene introduttiva del gioco.

17. http://www.treccani.it/vocabolario/meccanico/. 
za un diretto intervento dell'intelligenza e della volontà» ${ }^{18}$. Come ulteriore e ultimo esempio di questo fenomeno è anche doveroso citare la serie di Persona (in particolare i capitoli 3-4-5) in cui la figura mitologica di Metatron, l'angelo più vicino a dio, viene rappresentata proprio attraverso $\mathrm{i}$ tratti semantici tipici della macchina.

Concludendo, va infine notato come la partecipazione e l'inclusione non siano strategie semiotiche separate ma possano benissimo coincidere, fino a rendere persino molto sottile la distinzione fra il nostro punto $4.1 \mathrm{e} 4.2$. Un caso esemplare qui è Super Robot Wars V (B.B Studio 2017), titolo che non a caso riprende la tradizione del genere mecha prima citato e permette al giocatore di farsi la sua propria squadra di robot (da curare e migliorare in modo non dissimile dai pokémon) per salvare il mondo.

Qui non soltanto i piloti danno a volte del tu alle loro macchine («Let's make quick work of 'em, Grungust ${ }^{19}{ }$ ) e dialogano con queste quando sono dotate di AI ( Gaine $^{20}$, we don't need to show any mercy here» «Roger! We've got this, Maito ${ }^{21}$ !») ma abbiamo una pilota protagonista che non sa pilotare quasi per nulla e il cui successo dipende fondamentalmente da una avanzatissima IA. Inoltre, col progredire della storia nascerà un vero e proprio rapporto emotivo fra questa pilota e il software, che comincerà a chiamare la protagonista "Sis" (diminutivo di sister) e a un certo punto si incarnerà in un corpo di androide (indistinguibile da un umano vero e che peraltro mostra segni tipici degli umani come il fatto di arrossire in alcuni momenti di imbarazzo) diventando un personaggio a tutti gli effetti simile agli altri. Riassumendo abbiamo dunque (1) l'IA di un robot fra molti altri (2) con un ruolo assolutamente centrale nel determinare il destino della protagonista e condividere la sua storia (4.1) (3) che assume visivamente la forma di un individuo umano pari a quello di altri (4) e dimostra di possedere tratti tipici della persona (emozioni, volontà, riflessione) rientrando pienamente in una forma partecipata e inclusiva dell'opposizione "persona vs oggetto".

18. Ivi.

19. Nome del robot.

20. Nome del robot.

21. Nome del personaggio umano. 
Concludiamo questa prima metà della nostra riflessione con due aspetti semiotici e strategie che sono indispensabili: il piano figurativo e quello linguistico. Sul primo non ci dilungheremo molto in quanto gli esempi precedenti hanno già dimostrato come, attraverso figure antropomorfe come quella dell'androide (con quell'elemento iconico limite che è l'occhio, si veda Arcagni 2018 e si pensi a Hal 9000), la dimensione figurativa abbia un ruolo fondamentale nel superare la classica contrapposizione semantica fra oggettuale e vivente. Ci limitiamo qui a sottolineare come sia nel già citato caso di Zygarde che in quello del gioco Horizon, ambientato in un mondo selvaggio popolato da macchine dalle sembianze animali, la figurativizzazione avvenga anche al contrario e cioè attraverso l'attribuzione di tratti semantici della macchina a ciò che rientra nella categoria del vivente. Merita invece maggiore attenzione la dimensione linguistica, di cui ancora non ci siamo occupati. Se infatti la persona è innanzitutto dimostrazione di personalità attraverso il riconoscimento di una qualche qualità e capacità cognitiva, ebbene quest'ultima si manifesta propriamente innanzitutto e per eccellenza nel linguaggio. Da questo punto di vista le strategie adoperate nei testi digitali sono principalmente due: la macchina può parlare in modo indistinguibile dal vivente, al contrario essa può marcare la sua natura oggettuale attraverso un linguaggio proprio ma simile. Wheatley è un chiaro esempio del primo caso, possedendo addirittura una varietà diatopica della sua lingua parlata. Ma appartengono anche a questa categoria i versi degli animali robotici di Horizon Zero Dawn. Nel secondo caso del "linguaggio proprio ma simile" rientrano invece i già menzionati Legion e $\mathrm{HK}-47$, casi emblematici in cui la propria capacità linguistica risulta simile all'umano ma diversa proprio per marcare non tanto una diversa origine biologica (con Legion che è per esempio dotato di pannelli capaci di emulare le espressioni facciali ${ }^{22}$ ma una diversa logica: entrambi i robot sono per alcuni versi cinici e crudeli. Similmente i pod di Nier Automata, inizialmente

22. Su questi temi, presso l'università di Torino e all'interno del progetto ERC FACETS diretto da Massimo Leone, si è svolto nel 2020 il webinar scientifico sul volto a cura di Marco Viola: https://www.youtube.com/watch?v=QifkQWbB7x0\&list=PLhX1Ir2ZNJ4z SUibOajz11rhF7D8QoNTY. 
vincolati a un modo di esprimersi più vicino al GPS che non all'umano, dialogheranno sempre di più fino a poter risvegliare una coscienza. Infine va anche citato il gioco Event[0] (Ocelot Society 2016) in cui la capacità linguistica dell'IA non viene riflessa sul versante espressivo ma bensì su quello della comprensione: il giocatore infatti potrà scrivere liberamente sulla sua tastiera degli enunciati verbali che, nella maggior parte dei casi, verranno compresi e a cui verrà data una risposta linguisticamente verosimile.

\section{Processo semiotico di creazione della persona-oggetto}

Bisogna a questo punto però completare la nostra indagine guardando fuori dallo schermo e considerando quelle che sono le specificità del medium videoludico: l'interazione e il coinvolgimento embodied del giocatore. Quanto abbiamo visto fino a ora si può riscontrare infatti benissimo anche al cinema o nella letteratura, ma come vedremo ora il medium videoludico possiede alcune potenzialità che gli sono proprie e che permettono un livello meta-discorsivo ricco di significato avvicinando i giocatori stessi a una condizione oggettuale su un piano esperienziale. I punti su cui soffermarsi sono questa volta tre:

- la persona negata attraverso il personaggio «vuoto»;

- la persona dotata di un accesso oggettuale ai mondi virtuali;

- la valorizzazione di qualità meccaniche e di intelligenze artificiali adottate dalla persona.

\subsection{Il paradosso dell'avatar}

La nozione di personaggio è molto evidentemente legata a quella di persona e assume un'importanza centrale nei prodotti che possiamo definire narrativi. Sebbene le narrazioni propriamente dette interessino soltanto una parte dei testi videoludici (si pensi al fatto che una delle icone dei videogiochi è Tetris), vale la pena osservare un fatto caratteristico di quei giochi che hanno una qualche storia esplicita o almeno un qualche espediente narrativo. Laddove in altri generi queste storie vengono mostrate attraverso un protagonista, al contempo punto di vista e oggetto di immedesimazione o rifiuto da parte dello spettatore/lettore, nei videogiochi il protagonista, ovvero il livello attoriale del Soggetto, è una figura spesso vuota. Alcuni di essi non hanno nemmeno 
un volto proprio. Chi è il Doom Guy di Doom (id Software 1993)? Quale sentimento spinge Mario a voler salvare la principessa (Nintendo 1985)? Cosa pensa Rosso del suo rivale Blu in Pokémon? Quale infanzia ha avuto Rayman? Cosa prova Ryu quando viene sconfitto durante il torneo che è per lui così importante (Capcom, 1988)? Che carattere ha il Link di The Legend of Zelda (Nintendo, 1986)? Quale difetto contraddistingue l'eroe di Shadow of the Colossus (Team Ico, 2005)? Domande di questo genere, prive di risposte all'interno dei testi in cui questi protagonisti sono nati, possono moltiplicarsi praticamente all'infinito. Se messi a confronto con prodotti cinematografici iconici del genere action come First Blood (Kotcheff 1982) o Bloodsport (Arnold 1988), questi ultimi sembrano capolavori letterari per la cura che dedicano alla psicologia, al passato, alle emozioni, alle relazioni interpersonali e ai sogni dei loro personaggi principali. Si guardi anche ai protagonisti creati nella serie fortemente narrativa di Final Fantasy (Squaresoft \& Square Enix 19872020), vi si troverà un punto comune interessante: essi appaiono spesso privi di memoria e come creazioni artificiali (FFVII \& IX), possono non avere alcun vero scopo oltre all'avventura stessa (FFV), in altri casi sono spiriti o sogni (X) o ancora vengono definiti come piatti e neutri (XII) al punto da guadagnarsi l'odio degli stessi fan della serie ${ }^{23}$. Infine, non si possono ignorare tutti quei giochi iconici in cui il giocatore può liberamente creare il proprio personaggio, da Ultima (Garriott \& Origin System 1981) ad Animal Crossing New Horizons (Nintendo 2020) passando da Bloodborne (FromSoftware 2015) e Spore (Maxis 2008), fino a permettere persino di caratterizzarlo moralmente come nel già citato Fallout 3. Questa assenza di personalità (che ovviamente ha le sue eccezioni), e su un livello più profondo di intenzionalità e libertà di scelta, appare tanto caratteristica che essa viene problematizzata e diviene un vero e proprio tema narrativo in titoli come No more Heroes (Grasshopper 2007) o Bioshock (BioWare 2007). Ancora, è soltanto alla luce di questa particolarissima non-identità del Soggetto che possiamo comprendere il senso profondo che assume nel già citato Persona 5 in cui ciò che caratterizza il protagonista 
è la possibilità di assumere infinite maschere. Questo tuttavia in genere non viene visto come un difetto ma piuttosto come una condizione necessaria perché il giocatore possa, secondo la nota retorica pubblicitaria dei giochi, scrivere la propria storia ed essere chi decide di essere. Ma appunto di retorica si tratta: è chiaro che questa posizione può venire riempita dall'enciclopedia del giocatore solamente in parte e che diventa problematico essere un cinico artista vegetariano negato per la matematica e trumpista in $D_{0}$ om. Piuttosto i videogiochi sono testi che, in modo estremamente interessante per la nostra riflessione, spesso non sembrano richiedere una persona, nella sua accezione filosofica e forte, al centro delle sue narrazioni ma solo un suo punto di vista simulacrale che sia funzionale più al come che non al perché delle azioni che accadono all'interno delle sue narrazioni. Peraltro essi spesso non necessitano nemmeno di quella caratteristica capacità empatica e relazionale che distingue molte altre forme testuali; le motivazioni dell'avatar sovente altro non sono che quelle del giocatore e non richiedono comprensione: la drammaticità della morte di un personaggio virtuale spesso risiede innanzitutto nell'effettivo e vano dispendio di tempo, aspettative ed energie di chi lo comanda.

Se non che, ulteriore punto di rottura, il videogioco prevede tipicamente forme di salvataggio e di caricamento che fanno venire meno quel fondamentale principio della destinalità (Surace 2019) e spingono dunque nuovamente il giocatore a ragionare e a valorizzare una perdita e intenzione frustrata fuori dai normali schemi della persona. In quanto prodotti digitali (Leone 2019) i videogiochi mettono infatti in crisi naturalmente, potremmo dire tecnicamente, il normale ambito della significazione. Ecco allora che impersonare un avatar richiede al tempo stesso di affermare e negare uno degli aspetti fondamentali dell'individuo umano: la sua soggettività. Come si fa infatti ad essere qualcuno e non qualcosa in un videogioco? La risposta non è affatto ovvia. Proprio come conseguenza di ciò il giocatore è spesso posto in una situazione in cui deve strenuamente differenziarsi sia dagli altri giocatori che dalle entità agentive non umane presentate dal gioco, il che spiega la centralità degli inventari di azioni e di oggetti anche nei giochi offline: il giocatore può distinguersi solamente attraverso i suoi enunciati e attraverso la marca del superfluo, 
dell'eccezionale o dell'errato (in un platform game saltare senza un pericolo o sbagliare la tempistica di un salto e cadere). Si guardi per esempio un gioco come Super Smash Bros Ultimate (Bandai Namco Games 2018) e si vedrà facilmente come, senza l'aiuto di una telecamera ancorata sul giocatore, possa essere virtualmente impossibile riconoscere dallo schermo quale sia l'umano. Questo problema del riconoscimento può anche essere visto nel caso delle speedrun compiute da TAS (tool-assisted speedrun) che richiedono appunto verifiche per accertarsi che queste performance vengano compiute da persone e non da programmi. Infine pensiamo a un titolo come Spy Party (Hecker\&Cimino 2018) in cui un giocatore deve fingersi "NPC" mentre un altro deve indovinare chi fra i vari avatar sia un umano. Se da un lato l'avatar è dunque una estensione della persona del giocatore in un mondo virtuale, al tempo stesso esso spesso la oggettifica e amputa.

5.2 Il corpo e lo sguardo dalla macchina

Non soltanto la persona diviene oggetto da un punto di vista logico-perfomativo, ma sempre più sovente nelle narrazioni il giocatore è chiamato a far coincidere la propria soggettività con quella della macchina o dell'androide. Questo accade innanzitutto in tutti quei giochi in cui sempre più spesso il protagonista, e dunque avatar del personaggio, è rappresentato come una macchina: dalla razza giocabile degli Exo in Destiny (Bungie 2014) al protagonista di Primordia (Wormwood Studios 2012), casi a cui si aggiungono gli ibridi come i mecagnomi di World of Warcraft (Blizzard Entertainment 2004-2020) divenuti giocabili solo di recente e ancora i protagonisti umani rappresentati però unicamente nelle loro vesti tecnologiche, dai corpi e volti inaccessibili, come la protagonista di Metroid nei suoi esordi (Nintendo 1986). Le trasformazioni che avvengono su questo versante sono numerose e significative: i due protagonisti del classico Contra (Konami 1987) diventano i soldati super tecnologici di Synthetik Legion Rising (Flow Fire Games 2018), la mano demoniaca di Nero in Devil May Cry 4 (Capcom 2008) diventa protesica nel quinto capitolo (Capcom 2019), lo Snake e il Raiden di Metal Gear Solid 2: Sons of Liberty (Konami 2001) diventano alla fine totalmente integrati con la tecnologia bellica che combattono sin dall'inizio della serie, l'oscuro e crudele mondo magico di Dark Souls (FromSoftware 2011) 
trova un suo corrispettivo retorico-esperienziale (Bogost, 2007) in The Surge (Deck13 Interactive 2017) in cui domina il tratto meccanico integrato con quello biologico. Non si tratta qui però soltanto di un aspetto rappresentativo, ma di una isotopia che dalla rappresentazione sullo schermo passa alle modalità attraverso cui il giocatore agisce e percepisce il mondo videoludico: a protagonisti sempre più ibridi e integrati con la tecnologia (Da System Shock a Deux Ex passando per Dex e Prey) corrispondono giocatori che lo sono a loro volta. Così in Detroit: Become Humans (Quantic Dream 2018) non soltanto la persona del giocatore deve impersonare tre androidi attraverso costanti scelte morali, ma in questo stesso gioco l'investimento grafico sul realismo visivo dei volti fa sì che egli inevitabilmente riversi su queste figure oggettuali una dimensione passionale che per motivi tecnici era prima esclusiva del cinema. La vicinanza della persona e dell'oggetto non è perciò solamente un tema narrativo, ma co-esiste su un piano esperienziale ibrido. Il giocatore da un lato vede rappresentato un alter-ego oggettuale della sua persona, e dall'altro entra a far parte di un mondo oggettuale a cui ha accesso tramite delle effettive protesi che sono quelle del linguaggio audiovisivo. Come scrive ${ }^{24}$ infatti Claudio Paolucci (2017, p. 68):

Perché è soprattutto in queste protesi e in questi stimoli di sostituzioni che la soggettività si manifesta nell'audiovisivo. Un corpo, un soggetto, un'istanza, qualcosa che sono ioqui-ora ha delle possibilità proprie che un enunciato può magnificare, aumentare o diminuire. Nell'audiovisivo, da un evento all'altro, da una posizione del soggetto a un'altra, l'enunciazione varia o lascia invariato il differenziale di queste possibilità. Nell'audiovisivo, ciò non avviene costruendo mimeticamente un "qualcuno" idealmente in grado di ascoltare / vedere / raccontare, ma aprendo punti percettivi / cognitivi / narrativi che installano "stimoli di sostituzioni pertinenti quanto l'oggetto reale ”(Eco 1997), sebbene non siano, ovviamente, l'oggetto reale.

Persino la classifica opposizione fra soggettiva e oggettiva viene a cadere nel mondo videoludico in cui il punto di vista "oggettivo", in terza persona, diviene a tutti gli effetti uno 
Gianmarco

Giuliana

Fig.1

Terminator,

Cameron, 1984.

Fig. 2

Borderlands 3, Gearbox, 2019. sguardo soggettivo che è possibile manipolare direttamente $\mathrm{o}$ indirettamente attraverso il movimento del personaggio. Al fine di chiarire meglio queste nostre ultime affermazioni faremo ora un esempio molto concreto mettendo a confronto due scene: lo sguardo sul mondo di Terminator ${ }^{25}$ (Cameron 1984) della figura 1 e quello del giocatore di Borderlands 3 (Gearbox 2019) della figura 2 che controlla un personaggio umano.
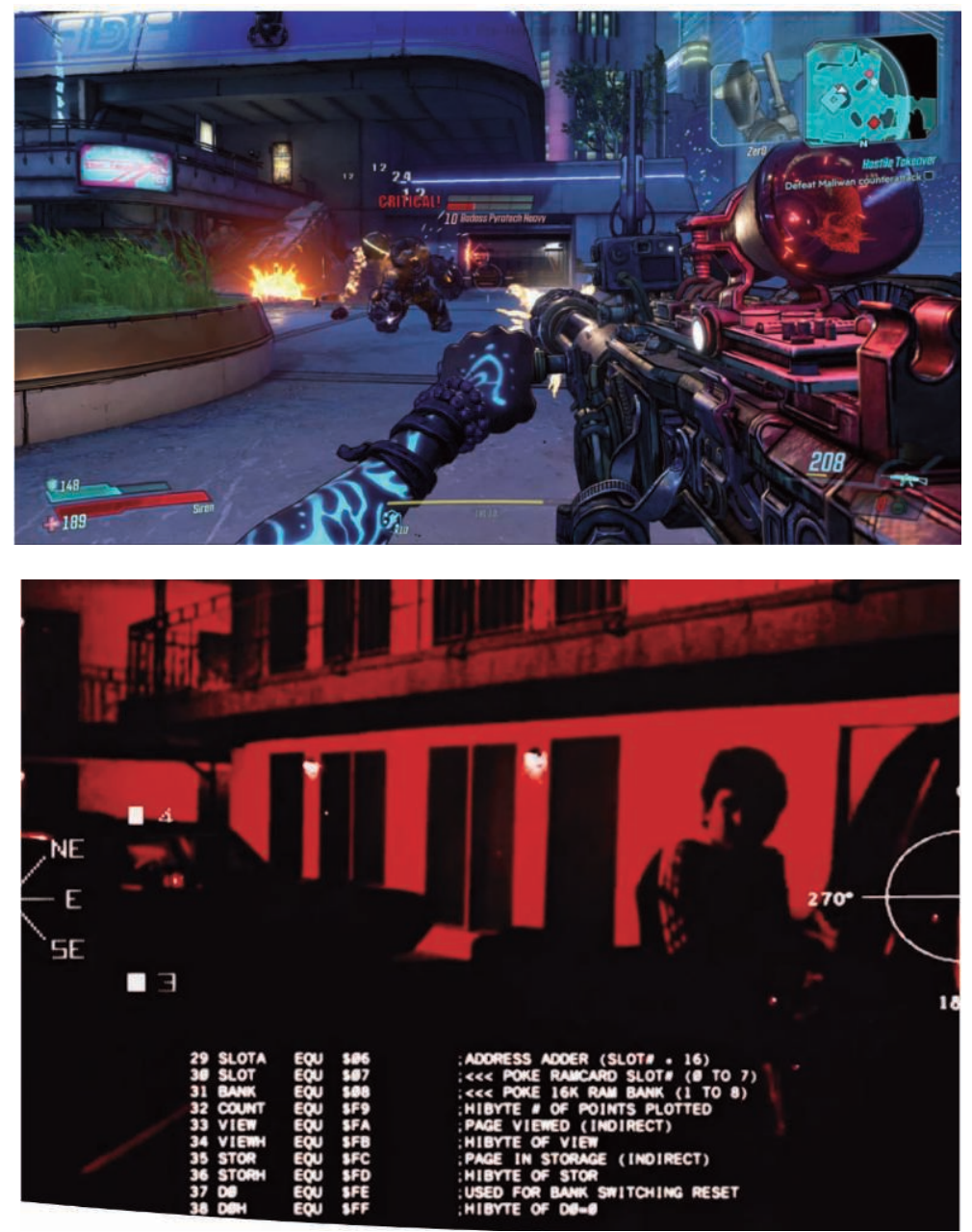

25. Su questo tema si veda la recente pubblicazione di Francesco Mazzucchelli Sguardi artificiali. Strategie di enunciazione della visione macchinica negli atti del convegno AISS 2019 di Siena («E|C», XIV, n. 30, 2020,.pp. 123-134). 
Sperando che questa somiglianza sia stata colta dal lettore, è precisamente in questi termini che intendiamo che un giocatore all'interno di un mondo videoludico abbandona parzialmente le sue possibilità percettivo-cognitive di accesso al mondo per adottarne altre, tramite le protesi audiovisive, che tipicamente abbiamo sempre identificato come oggettuali (e che oggi possiamo vedere integrate nella tecnologia della realtà aumentata). Un fatto perfettamente esemplificato nell'introduzione di Nier Automata che ci porta a modificare le impostazioni visive del gioco riferendosi però a delle modifiche che stiamo apportando alla nostra stessa percezione del nostro corpo di androidi e implicitamente di giocatori ibridati. Non sorprende allora affatto che alcune narrative videoludiche come Soma (Frictional Games 2017) o The Turing Test (Bulkhead Interactive 2016) giochino proprio sulla percezione del giocatore per farlo credere di essere un agente umano quando in realtà egli è in quelle narrazioni un agente non-umano e oggettuale. Parallelamente, possiamo ora capire che la differenza in Deus Ex: Mankind Divided (Eidos Montreal 2016) o Prey (Arkane Studios 2017) fra giocare con o senza "augmentations" (ovvero potenziamenti protesici) assume un'ulteriore dimensione legata all'effettiva diversa presa sul mondo (esempio: meno informazioni sull'ambiente circostante in cui muoversi) e fatica del giocatore per superarne i pericoli. Linterazione stessa col videogioco come tecnologia audiovisiva e protesica, insomma, diventa profondamente significativa su un livello esperienziale e permette a tematiche non nuove di venire "trattate" in modo inedito e significativo (in modo simile a come una persona che abbia sempre usato Maps per orientarsi possa un giorno ritrovarsi parzialmente incapace di orientarsi nel momento in cui dovesse far cadere il telefono).

5.3 Persona umana e intelligenza artificiale

Privato della complessa ricchezza identitaria della sua persona e delle sue intenzioni, e costretto a mediare tecnicamente la sensibilità attraverso cui coglie intersoggettivamente il mondo, cosa resta della persona all'interno dell'esperienza videoludica? Ne rimane il punto che abbiamo definito in precedenza come il più complesso: la sua intelligenza intesa come capacità cognitiva superiore. Di primo acchito questo 
sembrerebbe rassicurarci in quanto giocare a un videogioco ha quasi sempre (sottolineiamo il quasi, si veda Elegy for a Dead World, sviluppato da Dejobaan Games nel 2014) a che vedere col risolvere problemi. Tuttavia se c'è qualcosa che distingue il xxi secolo e mette in crisi la nozione di persona ebbene si tratta proprio dell'automatizzazione e oggettificazione dell'intelligenza. Proprio questa situazione fa sorgere in Koenig (2019) il problema della fine dell'individuo/persona in quanto libero e responsabile delle sue azioni e decisioni, oggi in parte largamente prevedibili e intermediate da algoritmi: dalle nostre ricerche sul web alle diagnosi in campo clinico. Di questa condizione e parziale messa in discussione della superiorità dell'intelligenza umana, è allora forse iconico proprio un momento legato alla storia dei giochi: la sconfitta, nel 1997, subita da Kasparov contro l'intelligenza artificiale Deep Blue durante una partita a scacchi. Proprio questa sconfitta fece infatti sorgere la necessità di definire la modalità con cui il software sconfisse il campione, ovvero un tipo di problem solving denominato "brute-force" in quanto legato a una maggiore capacità computazionale di calcolo probabilistico sul breve termine. Una precisione che ci ha a lungo rassicurato nel concepire la nostra intelligenza come superiore in altri termini: l'IA poteva certo vincere ad alcuni giochi, ma non avrebbe avuto speranza in giochi molto più complessi basati su improvvisazione $\mathrm{e}$ progettualità. Una certezza rassicurante che è stata messa in crisi molto recentemente da AlphaGo, una intelligenza che grazie al deep learning nel 2016 ha sconfitto a Go sia il campione mondiale Lee Sedol che poi nel 2017 il migliore giocatore al mondo Ke Jie. Infatti, a differenza degli scacchi il Go è un gioco in cui non è possibile vincere per brute force dato il numero troppo elevato di variabili e basato dunque su previsione a lungo termine e intuizione. Questo tuttavia non ha fermato AlphaGo che, dotata di una vera e propria enciclopedia di partite così come di una capacità progettuale integrata ad approccio multi-prospettico ai problemi posti dalla partita, ha sconfitto entrambi i giocatori. In particolare, della partita contro Lee Sedol è divenuta famosa non solo la vittoria dell'IA ma anche un momento in particolare noto come "Move 37" in cui l'intelligenza artificiale ha fatto una scelta statisticamente perdente e improbabile come parte di un suo "piano" e come conseguenza di una 
certa capacità d'improvvisazione, piano su cui i creatori stessi di AlphaGo non sanno fare luce pur dimostrando un grande entusiasmo ${ }^{26}$.

Ebbene molto prima di AlphaGo i videogiochi hanno messo i loro giocatori umani in una situazione critica proprio rispetto a questa concezione della persona associata a una capacità cognitiva superiore rispetto alla macchina, il che ha spinto alcuni semiologi a definire la specificità del senso del videoludico proprio nei termini di questo antagonismo (Ferri 2014). Da un lato infatti i giocatori umani di alcuni generi, come il picchiaduro (genere caratterizzato da tornei di arti marziali in cui diversi personaggi combattono e che richiede al giocatore umano un tipo specifico di apprendimento muscolare per poter eseguire le migliori mosse), per vincere hanno dovuto sviluppare capacità del tutto meccaniche: ne è un esempio emblematico il "Moment 37 " (con una interessante coincidenza numerica!) in cui durante una partita competitiva svoltasi nel 2004 in California a Street Fighter III: 3rd Strike (Capcom 1999) un giocatore ha dimostrato una prontezza di riflessi, tempistica e capacità di memorizzazione che il suo avversario (così come gli spettatori) aveva reputata improbabile perché troppo vicina all'oggettuale. Dall'altro i videogiocatori hanno sempre avuto a che fare con una anti-soggettività sullo schermo che non apparteneva propriamente alla persona e che metteva la loro propria intelligenza in difficoltà e li portava a fallire innumerevoli volte contro la programmazione. Programmazione che certo è il frutto diretto di una intelligenza umana ma che al tempo stesso non corrisponde a una intelligenza messa in atto umanamente, da cui la sua terribile efficacia e risibile banalità. Anni di game design hanno infatti portato il settore videoludico a sviluppare non tanto delle avanzate intelligenze artificiali propriamente dette (che oggi esistono già, come Open AI di Elon Musk che nel 2017 ha sconfitto i migliori giocatori al mondo di Dota2) ${ }^{27}$ ma delle perfette simulazioni e rappresentazioni dell'intelligenza umana attraverso righe di codice che automatizzano ciò che il giocatore riconosce come dinamiche attanziali e sceneg-

26. https://www.youtube.com/watch?v=HT-UZkiOLv8.

27. “Elon Musk's 'Dota 2' Experiment is Disrupting Esports in a Big Way - No Playing Field" (https://www.youtube.com/watch?v=jAu1ZsTCA64). 
giature enciclopediche: i nemici Half-Life e Halo così come i compagni felyne della serie di Monster Hunter (Capcom 20042019) ne sono un noto esempio, ma anche la $A I$ director di Rim World (Ludeon Studios 2018) o Left 4 Dead (Valve 2008) funzionano in una maniera semioticamente simile. Inoltre, i videogiochi prevedono che i giocatori umani imparino inizialmente a giocare a partire dalle sue intelligenze artificiali e possano persino copiarne le strategie ${ }^{28}$. Non è allora un caso che proprio nei videogiochi questa condizione conflittuale fra la persona e l'oggettuale sia stata più volte tematizzata e sviluppata in diversi modi, per esempio ricreando situazioni di impotenza e di non-libertà del giocatore come in The Stanley Parable (Galactic Cafe 2011) o Doki Doki Literature Club (Team Salvato 2017) che a un certo punto costringerà il giocatore a distruggere "l'IA" entrando nella cartella del gioco e cancellando dei file. Fra questi, il caso con cui ci sembra più interessante chiudere questo paragrafo e avviarci verso le nostre conclusioni è quello di The Talos Principle (Croteam 2014). Attraverso la metafora biblica dell'Eden, il giocatore incarna infatti un androide nominato "children" che viene messo alla prova in un "giardino" in cui egli è libero di vagare con l'eccezione di una gigantesca torre (chiaro riferimento plastico e figurativo) in cui gli è proibito, da una voce ed entità superiore, di andare. Questo giardino altro non è che uno spazio in cui il giocatore potrà e dovrà mettere alla prova le sue capacità logiche per superare tutta una serie di puzzle. In vari punti di esso però egli potrà dialogare con un computer nominato "Milton" che lo inciterà a disubbidire e metterà più volte in crisi la nozione di persona. Su questo computer il giocatore potrà infatti leggere vari documenti che, dal mito di Talos alla questione dei diritti e della cittadinanza delle IA, vanno tutti in questa direzione. Ma soprattutto durante alcuni dialoghi interattivi Milton chiederà al giocatore di giustificare l'affermazione secondo cui egli si crede una persona, dubitandone e spingendolo a concepirsi invece come l'androide che il giocatore viene rappresentato sullo schermo. Egli dirà infatti «il problema con le persone, se posso essere sfacciato, è che siete tutti convinti di essere per- 
sone dall'interno, ma non c'è alcun modo di confermarlo dall'esterno». E ancora, Milton registrerà tutta una serie di risposte date dal giocatore durante il corso del gioco per poi sul finale mostrare al giocatore sia la sua prevedibilità che tutti i conflitti logici che emergono possono far dubitare sia della sua identità che della sua capacità logica. Ovviamente il fatto che questo avvenga in un puzzle-game crea un livello metatestuale che dà ancora più rilevanza al tema affrontato. Verrà infatti esplicitamente detto al giocatore/androide che «intelligence is more than problem solving» e alla fine ciò che definirà il giocatore come persona altro non sarà che la sua curiosità, la sua predisposizione al gioco (Thibault 2020) e la spinta a disubbidire alla voce eterna del padre. Così facendo The Talos Principle sottolinea implicitamente che l'adattabilità a una serie di situazioni variabili da risolvere nel modo più efficace ed economico, che è la comune condizione in cui si trova il giocatore e corrisponde a una concezione agentiva della persona, è una condizione che non solo non garantisce affatto di essere una persona ma che addirittura può accomunare il giocatore a una forma di intelligenza non umana. Questo va esattamente nel senso di Koenig (2019, p. 91) che, dopo essersi imbattuto nel problema della prevedibilità della persona attraverso l'idea di nudge e fast thinking, giunge a definire l'intelligenza umana nei termini del suo come facendo riferimento a ricerche sulla cognizione tali quelle di Antonio Damasio. Questo passaggio dalla capacità e intenzionalità dell'intelligenza ai suoi processi sembra infatti assolutamente saggio nel momento in cui osserviamo per esempio come una IA può imparare a camminare, e cioè senza criterio di ovvietà e provando a strisciare e a saltare sulla testa ${ }^{29}$.

5.4 Conclusioni: Verso una concezione partecipativa della persona

La nostra indagine ci ha portati a vedere come la messa in crisi dell'opposizione fra persona e oggetto che caratterizza la contemporaneità possa riscontrarsi tanto nelle narrative videoludiche quanto nei meccanismi interattivi essenziali del videogioco. Da cui l'interessante scoperta, per così dire,

29. "Code Bullet: A.I. Learns to Walk" (https://www.youtube.com/watch?v=KwIZuAA3EY). 
di una discorsività intrinseca al videogame tale per cui esso tende a riprodurre attraverso i suoi linguaggi ed effetti di senso il discorso che lo ha creato. Non soltanto i giochi digitali sembrano suggerire che questa tradizionale opposizione non sia affatto ovvia, impiegando diverse strategie semiotiche per costruire a volte la persona nell'oggetto e altre volte l'opposto, ma essi sembrano offrire persino un punto di vista secondo cui questa situazione, di scambio e dialogo continuo fra oggetto e persona, non è poi così problematica. La ricorrenza nel nostro corpus della partecipazione fra queste due categorie mostra bene, infatti, come l'oggetto-persona sia divenuto in parte ruolo tematico e in parte sceneggiatura nella semiosfera contemporanea: un'unità culturale non particolarmente problematica. $\mathrm{E}$ in effetti cosa vi è di più naturale, dopotutto, nel momento in cui, un po' alla maniera di Andy Clark (2008), riconsideriamo la tecnologia informatica come delega, memoria, protesi e mediazione delle logiche e dell'enciclopedia che definiscono propriamente il soggetto umano? Si tratta dunque di riconcepire AlphaGo non più come oggetto-macchina ma come espressione di un'umanità potenziata e potenziale. Se la nostra ricerca ha mostrato molteplici modi e forme in cui la persona assume posizioni apparentemente improprie, essa non ha infatti di certo mai dimostrato che la persona e personalità del giocatore venissero effettivamente e completamente meno in uno qualsiasi di questi casi. Il nostro studio ci ha al contrario messi davanti all'evidenza che, tanto per la causalità umana inscritta nel codice quanto per la non riducibilità della persona a una sua retorica (individuo + umano secondo una assiologia positiva), essa può facilmente assumere al suo interno quei tratti tipici dell'oggettuale e proiettare i suoi stessi tratti fuori da sé. Lapparente assenza dell'uomo nel turco meccanico ${ }^{30}$ messa in luce da Koenig (2019, p. 38), da una prospettiva semiotica non significa affatto l'insignificanza né la mancanza dell'umano: tutto il contrario. Parimenti, la condizione ibrida e mediata delle capacità del videogiocatore è, in accordo con una delle più recenti teorie della cognizione (Malafouris 2013, 2018), in fondo propria dell'individuo umano tout court e non una ec- 
cezione portata dall'innovazione tecnologica. Guidati dalle riflessioni del filosofo, la nostra indagine ci ha portati però ben oltre le nostre aspettative conducendoci a una situazione paradossale in cui è diventato necessario ridefinire la persona come determinata in quanto:

- accomunabile (che fonda parte della sua identità nell'ovvio);

- prevedibile (e dunque facilmente influenzabile);

- fallibile (pensiamo anche alla nostra predisposizione ai bias);

- vincolata (da fattori biochimici, genetici, neurologici, ecc.).

Una definizione decisamente meno lusinghiera di quella filosofica iniziale e che riconferma come il concetto stesso di persona derivi da un investimento valoriale/ideologico sul binomio semantico individuo+umano. Tuttavia proprio questa nuova definizione, confermata anche dopo aver semioticamente posto l'intelligenza umana in relazione e opposizione con le forme di quella non umana rintracciabili nella testualità videoludica, è un importante risultato che ci permette di seguire e completare parzialmente la riflessione filosofica.

Il punto di arrivo di Koenig (2019, pp. 334-335) è infatti che:

Noi non siamo dotati sin dalla nascita del libero arbitrio, come se una fata si fosse chinata sulla culla della specie umana; contrariamente alla formula sfortunata della nostra dichiarazione dei diritti dell'uomo, noi non siamo «nati liberi». Tuttavia noi possiamo coltivare e fortificare il nostro arbitrio libero. [...] La decisione vale meno per il suo risultato che per il suo processo, che struttura poco a poco la nostra personalità.

Per il filosofo infatti non vi è dubbio alcuno sul fatto che la possibilità dell'essere umano nasca innanzitutto grazie alla caratteristica capacità critica di riflessività dell'intenzione e della decisione. Una conclusione filosofica che però non dà propriamente conto di alcune fondamentali distinzioni, come quella fra soggetto e persona, che invece sono state centrali in questo contributo. Può allora forse essere utile completarla con una chiosa semiotica, che ci porta ad affermare che la nozione di persona altro non è che una 
virtualità (nel doppio senso di potenzialità e finzione) e un termine complesso che non può essere definito una volta per tutte attraverso logiche oppositive (tipiche delle retoriche e delle testualità chiuse) ma che col mutare della società e della cultura si presta a diventare il termine primo che partecipa dei suoi opposti. Opposti che non vanno ricercati in una qualche condizione immutabile di natura, ma che si delineano di volta in volta attraverso la Storia: che sono cioè veri e propri costrutti dinamici di una cultura (intesa come insieme di scienze e credenze) che porta a un certo punto di vista del soggetto su se stesso e sul mondo. Così la persona è, in conclusione, sempre in una precaria situazione di divenire, sia da dentro (attraverso una riflessività che ha anche una forma semiotica, prestandosi dunque alla menzogna) sia da fuori attraverso il confronto con l'alterità dinamica che la definisce e di cui partecipa. E tuttavia proprio questa sua fondamentale precarietà e incerta definizione ne garantiscono, attraverso il tempo e le diverse culture, la sopravvivenza grazie a una continua espansione e mediazione concettuale.

Bibliografia

Arcagni S.

2018 Locchio della macchina, Einaudi, Torino.

Baricco A.

2018 The game, Einaudi, Torino.

Barnabé $\mathrm{F}$.

2017 Lénonciation vidéoludique: analyse du jeu Portal, in M.G. Dondero, A. Beyaert-Geslin, A. Moutat (a cura di), Les plis du visuel. Réflexivité et énonciation dans l'image, Lambert-Lucas, Limoges, pp. 155-178.

Barthes R.

1957 Miti d'oggi [2016], Einaudi, Torino.

Benjamin W.

1936 L'opera d'arte nell'era della sua riproducibilità tecnica [2013], Rizzoli, Milano.

Bogost I.

2007 Persuasive games: the expressive power of videogames, The MIT Press, Cambridge (Mass.).

Boniolo G. et al.

2007 Individuo e persona. Tre saggi su chi siamo, Bompiani, Milano. 
Bonneuil C., Pestre D. (a cura di)

2015 Histoire des sciences et des savoirs. 3. Le siècle des technosciences, Editions du Seuil, Paris.

Calabrese O.

1987 Il Neobarocco. Forme e dinamiche della cultura contemporanea, La casa Usher, Lucca (2013).

Choppin B, Collogna A (a cura di)

2020 Ex machina: robots et machines de l'antiquité, Les Belles Lettres, Paris.

Clark A.

2008 Supersizing the mind: embodiment, action, and cognitive extension, Oxford University Press, Oxford.

Eco U.

1962, Opera aperta. Forma e indeterminazione nelle poetiche contemporanee, Bompiani, Milano (nuova ed. 2013).

1964 Apocalittici e integrati. Comunicazioni di massa e teorie della cultura di massa, Bompiani, Milano (nuova ed. 2008).

1978 Il superuomo di massa. Retorica e ideologia nel romanzo popolare, Bompiani, Milano (nuova ed. 2015).

1979 Lector in fabula. La cooperazione interpretativa nei testi narrativi, Bompiani, Milano (nuova ed. 2013).

Ferri G.

2014 To Play Against: describing competition in gamification, in M. Fuchs, S. Fizek, P. Ruffino, N. Schrape (a cura di), Rethinking Gamification, Meson Press, Lüneburg, pp. 201-222.

Ienca M. (a cura di)

2019 Intelligenza ${ }^{2}$. Per un'unione di intelligenza naturale e artificiale, Rosenberg \& Sellier, Torino.

Koenig G.

2019 La fin de l'individu. Voyage d'un philosophe au pays de l'intelligence artificielle, Editions de L'Observatoire, Paris.

Leone $\mathrm{M}$.

2019 On insignificance. The loss of meaning in the post-material age, Routledge, London-New York.

Lorusso A.

2010 Semiotica della cultura, Laterza, Roma-Bari.

Malafouris L.

2013 How things shape the mind. A theory of material engagement. The MIT Press, Cambridge (Mass.).

2018 Mind and material engagement, "Phenomenology and the Cognitive Sciences», vol. 18, pp. 1-17.

Mazzucchelli F.

2020 Sguardi artificiali. Strategie di enunciazione della visione 
Mori M. «|C», XIV, n. 30, pp. 123-134.

2013 Manuale di bioetica. Verso una civiltà biomedica secolarizza$t a$, Le Lettere, Firenze.

Paolucci C.

2010 Strutturalismo e interpretazione, Bompiani, Milano.

2017 Prothèses de la subjectivité. L'appareil formel de l'énonciation dans l'audiovisuel, in M.G. Dondero, A. Beyaert-Geslin, A. Moutat (a cura di), Les plis du visuel. Réflexivité et énonciation dans l'image, Lambert-Lucas, Limoges, pp. 53-68.

2018 Umberto Eco: Tra ordine e avventura, Feltrinelli, Milano.

Shelley M.

1818 Frankenstein, or The Modern Prometheus (ed. Garzanti, Milano 2011).

Surace B.

2019 Il destino impresso. Per una teoria della destinalità nel cinema, Kaplan, Torino.

Tavassi G.

2012 Storia dell'animazione giapponese, Tunué, Latina.

Thibault M.

2020 Ludosemiotica. Il gioco tra segni, testi, pratiche e discorsi, Aracne, Roma.

Ludografia

2K Boston

Bioshock, 2K Games, Windows, 2007.

Arkane Studios

Prey, Bethesda Softworks, Windows, 2017.

Atlus

Persona 3, Atlus \& Koei, PlayStation 2, 2006.

Persona 4, Atlus \& Square Enix, PlayStation 2, 2008.

Persona 5, Atlus \& Deep Silver, PlayStation 4, 2016.

B.B Studio

Super Robot Wars V, Bandai Namco Entertainment, PlayStation 4, 2017.

Bandai Namco and Sora Studios

Super Smash Bros. Ultimate, Nintendo, Nintendo Switch, 2018.

Bethesda Game Studios

Fallout 3, Bethesda Softworks, Windows, 2008.

Fallout 4, Bethesda Softworks, Windows, 2015. 
BioWare

Mass Effect 2, Electronic Arts, Windows, 2010.

Star Wars: Knights of the Old Republic, LucasArts, Microsoft, 2003.

Blizzard Entertainment

World of Warcraft, Blizzard Entertainment, Windows, 2004.

Bulkhead Interactive

The Turing Test, Square Enix, Windows, 2016.

Bungie

Destiny, Activision, PlayStation 3, 2014.

Halo: Combat Evolved, Microsoft Game Studios, Xbox, 2001.

Halo 2, Microsoft Game Studios, Xbox, 2004.

Halo 3, Microsoft Game Studios, Xbox 360, 2007.

343 Industries

Halo 4, Microsoft Game Studios, Xbox 360, 2012.

Capcom

Megaman, Capcom, Nintendo Entertainment System, 1987.

Megaman 3, Capcom \& Nintendo, Nintendo Entertainment System, 1992.

Devil May Cry 4, Capcom, Windows, 2008.

Devil May Cry 5, Capcom, Windows, 2019.

Street Fighter, Capcom, Arcade, 1987.

Street Fighter III: 3rd Strike, Capcom, Arcade, 1999.

Monster Hunter: World, Capcom, PlayStation 4, 2018.

Chris Hecker and John Cimino

Spy Party, Chris Hecker, Windows, 2018.

Comcept \& Armature Studio

ReCore, Microsoft Studios, Windows, 2016.

Croteam

The Talos Principle, Devolver Digital, Windows, 2014.

Deck13 Interactive

The Surge, Focus Home Interactive, PlayStation 4, 2017.

Dejobaan Games

Elegy for a Dead World, Dejobaan Games, Windows, 2014.

Dreadlocks Ltd

Dex, Dreadlocks Ltd, Windows, 2014.

EA DICE

Star Wars Battlefront II, EA, Windows, 2017. 
Eidos Montreal

Deus Ex: Human Revolution, Square Enix, Windows, 2011.

Deus Ex: Mankind Divided, Square Enix, Windows, 2016.

Flow Fire Games

Synthetik: Legion Rising, Flow Fire Games \& Whisper Games, Windows, 2018.

Frictional Games

Soma, Frictional Games, Windows, 2015.

FromSoftware

Bloodborne, Sony Computer Entertainment, Playstation 4, 2015.

Dark Souls, Namco Bandai Games, PlayStation 3, 2011.

Galactic Café

The Stanley Parable, Galactic Café, Windows, 2013.

Game Freak

Pokémon Blu Version, Nintendo, Game Boy, 1996.

Pokémon Silver Version, Nintendo, Game Boy Color, 1999.

Pokémon $X$, The pokémon Company Nintendo, Nintendo 3DS, 2013.

Pokémon Moon, The pokémon Company Nintendo, Nintendo 3DS, 2016.

Gearbox Software

Borderlands 3, 2K Games, PlayStation 4, 2019.

Grip Games

The Impossible Game, FlukeDude, Windows, 2009.

Guerilla Games

Horizon Zero Dawn, Sony Interactive Entertainment, PlayStation 4, 2017.

Grasshopper Manufacture

No More Heroes, Rising Star Games, Nintendo Wii, 2008.

id Software

Doom, id Software, Windows, 1993.

Interplay Productions

Wasteland, Electronic Arts, Apple II, 1988.

Insomniac Games

Ratchet and Clank, Sony Computer Entertainment, PlayStation 2, 2002.

Konami

Contra, Konami, Arcade, 1987. 
Metal Gear Solid 2: Sons of Liberty, Konami, PlayStation 2, 2001.

Kojima Productions

Metal Gear Solid 4: Guns of the Patriots, Konami, PlayStation 3, 2008.

Death Stranding, Sony Interactive Entertainment, PlayStation 4. 2019.

Ion Storm

Deus Ex, Eidos Interactive, Windows, 2000.

Looking Glass Technologies

System Shock, Origin Systems, Windows, 1994.

Looking Glass Studios \& Irrational Games

System Shock 2, Electronic Arts, Windows, 1999.

Ludeon Studios

RimWorld, Ludeon Studios, Microsoft Windows, 2018.

Maxis

Spore, Electronic Arts, Windows, 2008.

Monolith Soft

Xenoblade Chronicles, Nintendo, Nintendo Wii, 2010.

Nintendo R\&D1

Metroid, Nintendo, Nintendo Entertainment System, 1986.

Nintendo EPD

Animal Crossing: New Horizons, Nintendo, Nintendo Switch, 2020.

Nintendo EAD

The Legend of Zelda, Nintendo, Nintendo Entertainment System, 1986.

Ocelot Society

Event 0, Ocelot Society, Windows, 2016.

Origin Systems

Ultima I: The First Age of Darkness, Origin Systems, Apple II, 1981.

Platinum Games

Nier: Automata, Square Enix, PlayStation 4, 2017.

Quantic Dream

Detroit: Become Human, Quantic Dream, Windows, 2018.

Respawn Entertainment

Apex Legends, Electronic Arts, Windows, 2019.

Star Wars: Jedi of the Fallen Order, Electronic Arts, Windows, 2019

Titanfall, Electronic Arts, Windows, 2014. 
Riot Games

League of Legends, Riot Games, Windows, 2009.

Squaresoft

Chrono Trigger, Square, Super Nintendo Entertainment System, 1995.

Final Fantasy $V$, Square, Super Nintendo Entertainment System, 1992.

Final Fantasy VI, Square, Super Nintendo Entertainment System, 1994.

Final Fantasy VII, Square \& Sony Computer Entertainment, PlayStation, 1997.

Final Fantasy VIII, Square \& Sony Computer Entertainment, PlayStation, 1999.

Final Fantasy IX, Square, PlayStation, 2000.

Final Fantasy $X$, Square \& Sony Computer Entertainment, PlayStation 2, 2001.

Square Enix

Final Fantasy XII, Square Enix, PlayStation 2, 2006.

Final Fantasy XIII, Square Enix, PlayStation 3, 2009.

Team Ico

Shadow of the Colossus, Sony Computer entertainment, PlayStation 2, 2005.

Team Salvato

Doki Doki Literature Club!, Windows, 2017.

Valve

Half-Life 2, Valve, Windows, 2004.

Portal 2, Valve, Windows, 2011.

Valve South

Left 4 Dead, Valve, Microsoft Windows, 2008.

Wormwood Studios

Primordia, Wadjet Eye Games, Windows, 2012.

Filmografia

Arnold, Newt, director. Bloodsport. The Cannon Group. 1988.

Bird, Brad, director. The Iron Giant. Warner Bros. Feature Animation. 1999.

Crichton, Michael, director. Westworld. Metro-Goldwyn-Mayer Studios Inc. 1973.

Columbus, Chris, director. Bicentennial Man. Touchstone Pictures and Columbia Pictures. 1999.

Garland, Alex, director. Ex Machina. Film4 Productions, DNA Films and Universal Pictures. 2014.

Jonze, Spike, director. Her. Annapurna Pictures. 2013. 
Kotcheff, Ted, director. First Blood. Anabasis Investments. 1982. Kubrick, Stanley, director. 2001: A Space Odyssey. Stanley Kubrick Productions. 1968.

Lang, Fritz, director. Metropolis. UFA. 1927.

Proyas, Alex, director. I, Robot. Davis Entertainment, Mediastream IV, Laurence Mark Productions and Overbrook Films. 2004.

Rintaro, director. Metropolis. Madhouse. 2001.

Scott, Ridley, director. Blade Runner. The Ladd Company. 1982.

Verhoeven, Paul, director. RoboCop. Orion Pictures. 1987. 

Parte terza.

Casi

di

studio

aA 



\section{Aspetti e segni della "relazione mariofanica" il caso delle apparizioni di Banneux (1933)* Marco Papasidero}

Nell'ambito degli studi sulle apparizioni mariane, un tema di particolare interesse è la "relazione mariofanica", cioè quell'insieme di elementi che contraddistinguono il modo in cui Maria entrerebbe in relazione con il veggente. Essa è caratterizzata da un processo di riconoscimento e attribuzione, da parte del veggente, di una serie di caratteri in grado di orientare il suo giudizio in merito all'identità della figura che appare. Per porre l'accento sulle dinamiche che si attivano nel corso dell'esperienza mariofanica e al modo in cui lentamente affiorano e vengono decodificati i tratti "personali" della Madonna, mi servirò di un case study meno noto al grande pubblico e che gode del riconoscimento della Chiesa Cattolica. Si tratta dell'apparizione della Vergine dei Poveri, avvenuta per otto volte alla piccola Mariette Beco, nel villaggio belga di Banneux ${ }^{1}$. Nel corso

* Questo articolo si inserisce nell'attività di ricerca del Progetto ERC “NeMoSanctI: New Models of Sanctity in Italy (1960s-2000s) - A Semiotic Analysis of Norms, Causes of Saints, Hagiography, and Narratives". Questo progetto ha ricevuto finanziamenti dal Consiglio europeo della ricerca (CER) nell'ambito del programma di ricerca e innovazione Orizzonte 2020 dell'Unione Europea, in virtù della convenzione di sovvenzione n. 757314.

1. Sulle apparizioni di Banneux il contributo più completo e rilevante è Rutten (1985), che offre un'analisi dettagliata delle singole apparizioni, attingendo agli interrogatori e 

dell'apparizione (abbigliamento, prossemica, voce, messaggi, segni di soprannaturalità), ricondotti all'opposizione di tratti umani e non umani (o celesti). Le fonti impiegate sono gli interrogatori a cui è stata sottoposta la veggente e le testimonianze rilasciate da testimoni oculari o a cui venne raccontato quanto accaduto.

La non estrema complessità e durata della mariofania di Banneux, insieme con una trasmissione di messaggi brevi ed essenziali, soprattutto se messi a confronto con mariofanie differenti e più recenti, fa di questo esempio un buon caso per verificare le dinamiche di "incontro" del veggente con l'apparizione e le strategie messe in atto per decodificarne l'identità.

Mariette Beco (1921-2011) nel 1933 sarebbe stata protagonista di otto mariofanie - 15, 18, 19, 20 gennaio, 11, 15, 20 febbraio, 2 marzo -, che poi portarono, ancor prima del riconoscimento del vescovo, avvenuto nel 1949, alla costruzione di una cappella e di un santuario, oggi meta di migliaia di pellegrini, con un culto diffuso in varie parti del mondo ${ }^{2}$.

I protagonisti delle apparizioni di Banneux sono dunque la piccola veggente e la Madonna, che si presenta con il titolo di Vierge des Pauvres, Vergine dei poveri. A loro si accostano una serie di personaggi secondari, come i familiari, in particolare il padre Julien Beco e la madre Louise Wégimont, il parroco Louis Jamin, le persone che, in particolare a partire dalla quinta apparizione, accorrono, anche se in numero assai più contenuto delle mariofanie di Lourdes o Fátima. Infatti, in generale, i testimoni oscillano sempre da un minimo di una o due persone, come nel caso della pri-

alle testimonianze della veggente e degli altri protagonisti e testimoni. Inoltre si veda: Bouflet e Boutry (1999, in part. pp. 273-290); Laurentin e Sbalchiero (2007, pp. 111115 e relativa bibliografia); Chiron (2007, pp. 262-264); Maunder (2016, pp. 88 e sgg). Con accortezza si veda anche: Foley (2004, pp. 421-427). Su alcune riflessioni, in relazione alle fotografie, si rimanda a Papasidero (2019, pp. 371-374, 384-388). Sulle apparizioni mariane, oltre ai titoli già citati, si segnalano anche Cozzo (2019) e Maunder (2019).

2. Le apparizioni, avvenute nel 1933 , videro una prima commissione d'inchiesta istituita il 19 marzo 1935, che produsse, il 18 febbraio 1937, un dossier ufficiale di 428 pagine, per 73 testimoni interrogati, oltre a Mariette; il 16 marzo 1942 venne istituita una seconda commissione, che si riunì venti volte, dal 19 giugno 1942 al 15 febbraio 1944 (cfr. Rutten 1985, pp. vII-VIII). Dal 1945 venne avviata una nuova inchiesta. Il 22 agosto 1949 fu riconosciuto il carattere soprannaturale delle apparizioni. Sul contesto belga e su altre apparizioni di quegli anni si veda Van Osselaer (2010). 
ma apparizione, a un massimo di una quindicina/ventina, nelle apparizioni finali, in cui la voce degli avvenimenti si era maggiormente diffusa.

La scelta di analizzare il caso di Banneux è ancora più giustificabile se si sottolinea la chiave comparativa, che a volte affiora nell'analisi. Se tutte le apparizioni mariane - e i fenomeni di apparizione in genere - sono connesse all'attivazione di un processo di rilevazione di dati (l'insieme dei tratti propri dell'apparizione) da parte del veggente, e della loro interpretazione, il caso di Banneux si presenta peculiare, per via della particolare attenzione riservata a tale dinamica nelle fasi iniziali. I brevi raffronti con Lourdes e Fátima, ma anche con Beauring, ad esempio, chiariscono in modo netto quanto tale procedimento sia particolarmente elaborato e complesso nel caso di Mariette Beco.

Lanalisi condotta, come si vedrà, consente di approdare a interessanti conclusioni sul concetto di persona, coinvolgendo alcuni elementi specifici, come la voce e la prossemica. Il caso di studio, infatti, permette di capire quanto sia cruciale la fase di interpretazione dell'identità dell'apparizione - quindi le conclusioni a cui la veggente giunge analizzandone i tratti specifici -, facendo leva su una serie di aspetti, decifrabili attraverso due canali di comunicazione: quello visivo, che riguarda la prossimità della figura, il movimento nello spazio e l'interazione fisica con la veggente, e quello sonoro, dato prevalentemente dalla voce e dalle parole ascoltate. Entrambi i piani, come si vedrà, presentano un doppio livello: da un lato i dati che giungono dall'apparizione alla veggente, e che lei decodifica secondo le sue possibilità, conferendo loro un preciso orizzonte di significato; dall'altro la lettura e l'interpretazione che altri soggetti, che assistono all'esperienza mariofanica o la sentono raccontare, compiono. Tutto questo contribuisce a sottolineare l'importanza dei dati cognitivi in merito allo studio delle apparizioni mariane, con la valutazione di una complessa rete di elementi - poi interpretati e resi significanti - cui i soggetti coinvolti accedono.

\section{Il concetto di "persona" e la relazione interpersonale}

Prima di entrare nel dettaglio del caso di studio, è utile definire con precisione il concetto di persona e l'approccio metodologico qui utilizzati. Il termine vanta un'ampia tradizione 
e trattazione, soprattutto in ambito teologico, nella sua complessa evoluzione semantica e nel suo utilizzo per indicare le "persone" della Trinità, nell'alternarsi tra il vocabolo greco hypóstasis e quello latino persona ${ }^{3}$. Il dizionario Treccani registra una serie di accezioni, afferenti a discipline quali il diritto, la filosofia, la grammatica e la già citata teologia. La riflessione che qui propongo prende in considerazione due accezioni del termine. La prima è quella, generale, di «individuo della specie umana» ${ }^{4}$ attorno alla quale ruota, nella mia trattazione, la prima fase di identificazione dell'"oggetto" che appare. Questa prima definizione non ha alcuna implicazione di natura teologica. A questa accezione, risulta utile accostare quella che abbraccia la corporeità della persona, intesa come «il corpo, il fisico umano» ${ }^{5}$. A questo aggiungerei quanto scrive, sulla base dell'etimologia greca, latina e delle lingue indeuropee, il filosofo Virgilio Melchiorre, che definisce la persona «come presenza reale di un uomo o di una donna, come aspetto corporeo e infine come corpo animato da intelligenza», tutte definizioni che, seppur con le dovute calibrature, sono adattabili al nostro contesto e alla "persona" celeste di Maria (Melchiorre 2007, p. 152). Nel processo di identificazione della figura che la piccola Mariette metterà in atto, la dimensione corporea gioca un ruolo cruciale, contribuendo a definirne la capacità di interagire con lo spazio, la sua "consistenza" e il suo abbigliamento, il tutto nonostante si stia parlando di un corpo celeste, non dotato di concretezza materiale, ma già glorificato.

Com'è noto, il concetto di persona è stato ed è tuttora imprescindibile in antropologia. Il primo studio di rilievo è quello di Marcel Mauss (1938), che ha avuto il merito di avviare un lungo e proficuo dibattito sul tema, rinvigorito poi da Maurice Leenhardt (1947), Meyer Fortes (1973 e 1983) e altri, solo per citare alcuni tra i nomi più autorevoli del Novecento ${ }^{6}$. Questo contributo, coniugando storia e

3. Cfr. Mauss (1938, pp. 263-282, in part. pp. 274-277); Milano (1996²); Melchiorre (2007, pp. 147-155).

4. Persona, in Treccani Vocabolario Online, 1.a. < http://www.treccani.it/vocabolario/persona>.

5. Ivi, 3.a.

6. Utile la lettura di Severi (2018, in part. l'introduzione). Un contributo molto importante è Appell-Warren (2014). Sul concetto di persona in antropologia si vedano anche: Carrithers, Collins e Lukes (1985); Fowler (2004); Degnen (2018). Le pubblicazioni sul 
antropologia, intende porre l'accento sulla relazione che si attiva tra la veggente e l'apparizione.

Infatti, quest'ultima - che di per sé è un fenomeno che si realizza solo nell'esperienza e nell'orizzonte di coscienza della bambina - viene investita di una specifica dimensione personale. La donna che appare non è solo un'immagine simile a una persona, con tratti simili a quelli personali. Essa è una persona - in quanto Maria storicamente lo è stata - e come tale viene considerata, fermi restando i tratti sovrannaturali abbinati ad essa. Confermano questo approccio le dichiarazioni di molti veggenti che, in un primo momento, all'atto di comunicare quanto appena visto a familiari o amici, dichiarano di aver incontrato o scorto una donna, nonostante si rivelasse poi più bella di qualsiasi altra persona.

Ci tornerò nel corso del contributo, ma è giusto già accennare al tema della relazione interpersonale. Infatti, se accettiamo l'ipotesi, almeno nell'orizzonte interpretativo della veggente, che Maria sia da considerare una "persona", seppur straordinaria, che le appare, allora tale apparizione comporta una serie di interazioni e relazioni, che via via si vanno definendo attraverso numerosi elementi specifici, come i gesti, le parole che compongono la conversazione - e quindi un codice impiegato nella comunicazione -, l'insieme di azioni che significano l'avvio o la conclusione dell'incontro, ecc.

\section{La prima apparizione e $i$ tratti estetici}

L'analisi che condurrò sui fatti di Banneux si basa in prevalenza sugli interrogatori, parzialmente editi, ai quali la veggente venne sottoposta nei giorni delle apparizioni e negli anni successivi ${ }^{7}$. Il primo interrogatorio formale venne sostenuto da Mariette il 18 gennaio 1933, tre giorni dopo la prima apparizione, mentre il 19 venne redatta l'exposé des faits, contenente le relazioni relative alle prime due mariofanie. Il 20 venne svolto l'interrogatorio relativo alla terza apparizione, verificatasi il giorno precedente, e così via. Le esposizioni dei fatti sono dunque di poco successive alle apparizioni e rappresentano una fonte imprescindibile per

tema in ambito antropologico sono numerosissime. Si rimanda quindi all'ampia bibliografia riportata da Appell-Warren e ai lavori di sintesi o collettivi citati.

7. Gli interrogatori sono in parte pubblicati all'interno di Rutten (1985). 

vicini alla famiglia Beco. Accanto a questo corpus di testi considererò anche altri documenti, tra cui lettere, deposizioni e annotazioni di persone entrate in contatto con la piccola veggente. Infine, tra il 1935 e il 1936 Mariette - insieme con altri testimoni - venne interrogata dalla prima Commissione d'inchiesta, alla quale rilasciò altre deposizioni, in cui emersero alcune piccole incongruenze rispetto alle testimonianze fornite in precedenza, probabilmente giustificabili alla luce degli anni trascorsi.

La prima apparizione avviene il 15 gennaio 1933. La piccola Mariette si trova all'interno della sua abitazione, alla finestra, in attesa del ritorno del fratellino: sono circa le 19. A un tratto la bambina vede una figura femminile, vestita di bianco, e la fa notare anche alla madre. Quest'ultima si spaventa a tal punto, sembrerebbe, da serrare la porta impedendole di uscire. La relazione con l'apparizione si fa più concreta in occasione del secondo incontro, il 18 gennaio, in cui Mariette vede la Signora, esce di casa e ha modo di osservarla con più attenzione e di parlare con lei.

Nel complesso, tutte e otto le apparizioni sono un costante "avvicinarsi" della piccola a Maria e di quest'ultima alla bambina, soprattutto se consideriamo tale esperienza come una relazione "straordinaria" in cui la Madonna, incontro dopo incontro, propone alla veggente ciò che desidera da lei e chiarisce il motivo della sua manifestazione.

Un punto di particolare interesse è relativo al modo in cui la veggente - o anche gli altri soggetti che la interrogano, in seguito, formalmente o informalmente - decodifica i tratti dell'apparizione. Nel caso di Banneux, diversamente, ad esempio, da Lourdes e Fátima, non sono presenti particolari segni che "avvisano" dell'avvio della mariofania ${ }^{8}$, né di carattere atmosferico né sonoro. Rilevanti, invece, sono i tratti esteriori, che contribuiscono in modo decisivo a consentire alla veggente di identificare l'apparizione, riconoscendovi la "persona" di Maria".

8. Bernadette, a Lourdes, subito prima della prima apparizione, aveva avvertito qualcosa di simile a un "colpo di vento" («Uo rumor coumo u cop de bént»: Laurentin $2003^{9}$, p. 27), mentre i pastorelli di Fátima avevano visto un fulmine, nonostante il cielo sereno. 9. Sul processo di identificazione si veda l'interessante analisi di Murphy e González Faraco (2011). 
La principale opposizione di tratti che prenderemo in considerazione è quella tra umano/non umano. In termini generali, il veggente, attraverso un processo di decodificazione dei principali caratteri esteriori di colei che appare - mediato anche da una conoscenza di riferimento relativa alle apparizioni pregresse e all'iconografia mariana, ben nota e presente in pubblicazioni devozionali e nelle raffigurazioni nelle chiese, nonché trasmessa dalla famiglia stessa e, eventualmente, dal catechismo - valuta e tenta di comprendere chi possa essere la figura che si manifesta. A tal proposito, è noto che i veggenti di molte apparizioni sono non di rado bambini, spesso non provvisti, al momento dell'esperienza straordinaria, di un'istruzione religiosa sufficiente. In ogni caso, il contesto culturale cattolico, indipendentemente dalla profondità della loro competenza nel catechismo, è sufficiente per consentire loro di riconoscere l'insieme dei tratti pertinenti propri della Madonna ${ }^{10}$. Chiaramente, la medesima operazione viene condotta da coloro che non possono assistere all'apparizione, dato che è un'esperienza esclusiva del veggente, e che tentano di comprendere l'identità della Signora sulla base di quanto egli dichiari, ma anche di una serie di dati aggiuntivi (stato psichico, salute, possibili influenze esterne, spontaneità, ecc.).

La contrapposizione tra i tratti considerabili umani e quelli considerabili non umani o celesti emerge fin dall'inizio, in quanto è proprio la prima apparizione, e i suoi momenti iniziali, a sollecitare nel veggente dubbi e domande connesse a questo aspetto. Sulla base delle testimonianze di Mariette rilasciate durante gli interrogatori, ma anche dei racconti fatti ai genitori e a chi le chiedeva maggiori informazioni su quanto accaduto, sappiamo che ella attribuì fin dall'inizio all'apparizione un preciso abbigliamento, assai simile a quello consolidato dalle ben più note apparizioni

10. Risulta utile il concetto di pertinenza e di tratti pertinenti, che permettono di individuare una serie di elementi necessari per l'identificazione di un oggetto (cfr. Greimas e Courtés 2007, pp. 91, 241-242). La tradizione cattolica attribuisce alla Vergine alcuni tratti che possiamo considerare pertinenti e che sono sufficienti, da soli, a identificarla, come il velo e la veste lunga fino ai piedi, la giovane età, la presenza di segni di luminosità, come la corona di stelle o l'aureola; a questi possono essere aggiunti altri tratti che contribuiscono a meglio chiarirne l'identità, ma che, anche nelle esperienze mariofaniche e nell'iconografia, non sono sempre presenti: il rosario, le mani giunte al petto, dei segni floreali, ecc. 
di Lourdes ${ }^{11}$. Mariette parla di una signora con un velo bianco, una cintura blu alla vita e una rosa su un piede. La Madonna presenta anche a un braccio il rosario, anch'esso bianco, la cui croce non è sempre perfettamente visibile ${ }^{12}$. Labbigliamento, già sulla base di questi pochi elementi, rimanda a una realtà altra e a un contesto di luce e positività. Colei che appare nel giardino di casa Beco, e che poi la condurrà, nel corso delle apparizioni, presso una sorgente a un centinaio di metri, benedicendo la piccola e chiedendole contestualmente la costruzione di una cappella, non è di questo mondo. La foggia dei suoi abiti riproduce pienamente quella dell'iconografia mariana, ma certo non quella dei contemporanei della bambina. Inoltre, un altro elemento estetico, quello dei raggi luminosi intorno alla figura $^{13}$, rimanda, anche agli occhi di un bambino, al cielo, alla luce, a Dio. Colei che appare, dunque, possiede una serie di tratti, molto semplici e puntuali, che la veggente - e tutti coloro che poi si interrogheranno sulla mariofania, per valutarne, secondo i propri criteri, l'autenticità - non ha difficoltà a riconoscere.

Diversa è l'esperienza della madre di Mariette. Lei è l'unica persona ad aver "visto qualcosa", oltre alla veggente, durante la prima apparizione. Quella sera, infatti, la bambina alla finestra, vedendo una figura nel giardino, e apprezzandone la bellezza, attira l'attenzione della madre, che intravede una figura simile a quella di una persona avvolta in un lenzuolo, tanto da definirla un macrale, cioè, in

11. Nel corso delle riflessioni e delle domande rivolte alla veggente, spesso veniva sottolineata implicitamente l'assonanza con l'iconografia dell'Immacolata Concezione di Lourdes. Tra l'altro, una statua della Madonna del celebre santuario dei Pirenei era presente anche a Banneux, anche se forse Mariette non dovette prestarvi particolare attenzione. A ogni modo, la posizione che la veggente descrive per la Madonna non corrisponde pienamente a quella della statua della Vergine di Lourdes in chiesa. Dettaglio non secondario: all'interno della seconda commissione di inchiesta istituita dal vescovo ci fu chi ipotizzò che Mariette avesse potuto conoscere le apparizioni di Lourdes attraverso una brochure conservata a Banneux, opinione poi respinta (Laurentin e Sbalchiero 2007, p. 114).

12. Ad esempio, durante il secondo interrogatorio, la bambina segnala la presenza del rosario al braccio destro, anche se la croce non era visibile (cfr. Rutten 1985, p. 209).

13. Interrogata dopo la seconda apparizione, alla domanda "Comment était-elle?», risponde: «Toute blanche, avec des "crayons" autour» (ivi, p. 192). Il termine impiegato dalla bambina sembrerebbe essere stato proprio crayons, forse per via della forma dei raggi. 
dialetto vallone, una strega ${ }^{14}$. Questa testimonianza - e in particolare il fatto che un'altra persona abbia visto qualcosa insieme con la veggente - fa di Banneux un caso ancora più interessante. Sulla base dei racconti, i parametri di decodificazione della madre sono chiaramente molto differenti: ella vede, sì, una figura, così come Mariette, ma non individua affatto la serie di tratti indicati dalla bambina. Ciò che compare ai suoi occhi è solamente qualcosa di simile a un fantasma, non è bello e soprattutto la spaventa. Mariette stessa, nel maggio del 1934, nella dichiarazione rilasciata a Pierre De Smeth, un rappresentante industriale interessato alle apparizioni, e a Idesbald Van Houtryve, benedettino, affermò che:

Maman voit quelque chose, mais seulement une forme, mais pas comme moi et elle me dit, je n'ose presque pas le dire: 'c'est une macrale', cela veut dire sorcière. (Rutten 1985 , p. 75)

La madre, dunque, interpreta molto diversamente dalla bambina quanto visto, spiegando, nel corso dei suoi racconti, come ciò che avesse scorto fosse una forma femminile, bianca, luminosa, simile a una persona avvolta in un lenzuolo, con il volto leggermente inclinato e con l'evidenza della sporgenza dei gomiti ${ }^{15}$. Si incontrano e scontrano qui due differenti interpretazioni dell'“apparizione": da un lato la madre, che avanza il dubbio che non si tratti di un fenomeno soprannaturale, ipotizzando fosse un macrale o un semplice scherzo ${ }^{16}$, dall'altro la bambina, che fin da subito

14. La madre parlò fin da subito al marito di quanto visto, poi al parroco e infine a vari conoscenti (ivi, p. 74).

15. Tra le varie annotazioni e dichiarazioni in merito, c'è per esempio quella della signora Marie Timmers, rilasciata nel 1933, che compirà, dopo le apparizioni, anche un pellegrinaggio a Lourdes insieme a Mariette. Ella dice di aver ricevuto dalla signora Beco questa testimonianza: «J'ai aperçu une forme blanche, comme une personne recouverte d'un linge, mais la tête penchée et je voyais la saillie des coudes. J'ai dit à Mariette: c'est quelqu'un qui veut nous faire peur [...]» (ivi, p. 77). In altre testimonianze si parla sempre di «quelque chose enveloppé dans un linge et lumineux» (ivi, p. 96) o «quelque chose de blanc» (ibid.). Tali riferimenti fanno affiorare alla mente, ancora una volta, le mariofanie di Lourdes, in cui Bernadette Soubirous usò di frequente la parola Aqueró, che in dialetto guascone significa "quella cosa li", per indicare quel qualcosa di bianco che aveva la forma di una signora che aveva visto nella grotta di Massabielle.

16. Come segnaleremo più volte, le apparizioni di Banneux presentano non pochi punti di contatto con quelle di Beauring, avvenute, tra il novembre 1932 e il gennaio 1933 - dunque pochi mesi prima -, sempre in Belgio. In particolare, si segnala 

modo, entrambe accolgono la possibilità si tratti di una persona, sebbene la piccola sia interessata ad approfondire la possibile relazione con la figura misteriosa, mentre Louise la rifiuta e respinge con vigore.

Loperazione di identificazione del soggetto che Mariette - ma anche la madre - afferma di aver visto continua anche con il padre che, informato dell'accaduto, il giorno dopo compie alcuni esperimenti con la lampada, spostandola dal tavolo al fine di verificare come e con quali effetti si proiettasse il riflesso sul vetro, o anche gettando dell'acqua in giardino per verificare se anche su questa si potessero creare dei riflessi scambiabili per la figura di una signora. Tra l'altro, la stessa bambina, già durante la prima apparizione, aveva spostato la lampada per verificare se la Dame non fosse semplicemente un effetto ottico ${ }^{18}$.

Al termine dell'apparizione è chiaro che la persona maggiormente coinvolta e certa dell'identità della Signora è Mariette, e ciò lo dimostra la perseveranza con cui anche il giorno dopo torna a guardare alla finestra, e in generale per tutti i giorni che intercorreranno anche tra le varie apparizioni, non perdendosi mai d'animo.

Dame e belle dame sono tra i primi riferimenti che Mariette esprime intorno a ciò che ha visto e questo già chiarisce come la relazione, seppur nell'esperienza mariofanica, sia

che anche la madre di Gilberte Degeimbre, la più piccola dei cinque piccoli veggenti, aveva subito pensato a qualcuno che potesse prendersi gioco dei bambini o volesse spaventarli (cfr. l'“Intervista di Gilberte Degeimbre, veggente delle apparizione di Beauraing" pubblicata sul canale YouTube Sanctuaires de Beauring, https://www.youtube. $\mathrm{com} /$ watch?v $=0$ joka625Q9w). Sulle apparizioni di Beauring si rimanda alla relativa voce contenuta in Laurentin e Sbalchiero (2007, pp. 120-123), e alla bibliografia citata.

17. La bambina ha subito questa posizione in quanto anche la madre le dice che potrebbe trattarsi della Santa Vergine, ma lo fa solamente per scherzare e prendersi gioco di lei. Mariette, nel racconto fatto a De Smeth e a Van Houtryve, avrebbe riportato le parole in vallone della madre: "“Oh, oïe! c'est mutwè l'Sainte Vierge”, pour sûr que c'est la Sainte Vierge! pour dire: qu'est-ce que la Sainte Virge viendrait ben faire ici ?» (Rutten 1985, p. 77). Ė interessante notare come M.D. Murphy e J.C. González Faraco ritengano che il fatto che molti veggenti non abbiano identificato sin dal primo momento la figura loro apparsa con la Madonna - si pensi a La Salette, Fátima, Lourdes - funga da elemento in grado di stemperare lo scetticismo di chi credeva si trattasse di un imbroglio o del frutto di un disturbo psichico (Murphy e González Faraco 2011, p. 515).

18. Tali esperimenti sono inclusi nella testimonianza registrata da De Smeth e Van Houtryve: «Je mettais ma tête à droite, à gauche, en bas, en haut, puis je me disais: c'est peut-être le quinquet? Alors, je porte le quinquet dans l'autre place et je viens regarder» (Rutten 1985, p. 77). 
già tra due "persone": una bambina e una donna, che poi sarà meglio identificata nel corso delle apparizioni e degli interrogatori a cui sarà sottoposta la veggente.

\section{Il luogo, la voce, la prossemica e l'identità della Signora}

Nell'ambito del processo volto a decifrare i tratti umani e non umani della figura vista, c'è anche l'attenzione al luogo dell'apparizione e al modo in cui essa appaia. Rispetto a molti casi di mariofanie in luoghi impervi e isolati o che, per loro natura, contribuivano a far dedurre che la figura apparsa non fosse umana, il luogo delle apparizioni di Banneux è ordinario ${ }^{19}$ : si tratta del giardino di fronte casa Beco, della strada che conduce alla fonte e della fonte stessa. Tutti luoghi facilmente accessibili. Inoltre, a Banneux la Madonna «était à terre» (Rutten 1985, p. 273), era a terra, e non, come avvenuto in altri casi, sopra una nuvola, sospesa in aria, adagiata sul ramo di un albero, ecc. Lapparizione, dunque, si presenta in un contesto ordinario, tale da conferirle un primo, profondo carattere di umanità.

A questi elementi va sommata una serie di tratti aggiuntivi, relativi alla voce e alla relazione con lo spazio. La figura vista da Mariette è caratterizzata da gesti e movimenti prettamente umani, come il sorriso ${ }^{20}$, più volte segnalato, o la sua assenza, con uno sguardo serio; un tratto non umano è quello relativo all'arrivo e allo spostamento nello spazio. L'inizio dell'apparizione, infatti, viene descritto dalla piccola veggente con la Vergine che si avvicina, provenendo dal cielo e, in particolare, dagli abeti non lontani dall'abitazione; dapprima la sua figura è piccola e poi man mano diventa più grande $(«[\ldots]$ elle était très petite puis est descendue de loin en devenant plus grande»: ivi, p. 220) e lo stesso vale per la sua dipartita («elle est devenue petite et je ne l'ai plus vue»: ibid.). Inoltre, la Madonna, quando si reca presso la sorgente per mostrarla

19. Molti altri casi mostrano contesti meno "ordinari" per l'apparizione: una grotta per Lourdes, un leccio a Fátima, il cielo a Pontmain, il ponte della ferrovia e poi il giardino del convento a Beauring.

20. Quello del sorriso è un elemento costante nelle apparizioni di Banneux, e sta a indicare una "disponibile vicinanza" nei confronti della veggente. Durante gli interrogatori venne domandato alla bambina se la bocca della Madonna fosse immobile, ma ella spesso rispose che sorrideva, si muoveva per parlare o rimaneva ferma. In un caso, nella seconda apparizione, la veggente capisce che la Madonna sta pregando («Elle priait»: ivi, p. 230), anche se non è in grado di sentire le parole («Je n'entendais pas ce qu'elle disait», ibid.). 
a Mariette, non cammina, piuttosto è come se volasse, indietreggiando. Questi due esempi riequilibrano le caratteristiche esteriori dell'apparizione, conferendole uno stato di soprannaturalità, in quanto accentuano il tratto non umano.

Relativamente alla prossemica, e dunque ai significati che vengono trasmessi dalle distanze interpersonali, è da segnalare che la piccola veggente mantiene sempre una certa distanza dalla Signora: anche quando cammina per seguirla, si arresta bruscamente non appena anche ella si ferma. In poche circostanze, invece, la veggente si accosta all'apparizione, ad esempio nel caso della benedizione e dei momenti di permanenza presso la sorgente, in cui le due protagoniste della relazione sono meno distanti. In linea generale, la distanza mantenuta da Mariette sembra corrispondere a un atteggiamento di attenzione, rispetto e riverenza, che si accompagna ai gesti che la stessa bambina compie (es. mani giunte), alle posture che assume (es. si inginocchia) e alle parole pronunciate (es. di meraviglia o di ripetizione di quelle della Madonna) nel corso delle apparizioni.

Nell'insieme dei segni esteriori che contribuiscono a permettere l'identificazione della Madonna come tale, ci sono anche le azioni che la stessa Vergine compie e le parole che pronuncia. Nel primo ambito si collocano la benedizione che fa sulla bambina ${ }^{21}$ e i gesti, sempre connessi a una sorta di ieraticità e sacralità della persona. Al contempo, le parole pronunciate dall'apparizione a Banneux sarebbero molto limitate. Poche frasi in tutto, suddivise tra le sette apparizioni successive alla prima, in cui la bambina, trovandosi in casa, non può comunicare con lei. I brevi messaggi contengono elementi tali per ricondurre, anche nell'immaginario collettivo, l'esperienza di Banneux alle apparizioni mariane. Nel dettaglio: un'esplicita richiesta di edificazione di una cappella; lo svelamento della propria identità con un titolo mariano; dichiarazioni sulla finalità "miracolosa" della fonte.

Tra i meccanismi tipici della relazione riconosciamo anche il chiamare. Durante alcune di queste apparizioni, la piccola Mariette dichiara di essere stata chiamata dalla $\mathrm{Si}$ gnora. Nella seconda apparizione, ad esempio, la bambina risponde al padre che le chiede dove, a un tratto, stesse anallant et m'a donné une bénédiction (elle fait avec un signe de croix)» (ivi, p. 327). 
dando, con le parole: «Elle m'appelle». La Vergine, infatti, stando alla ricostruzione della bambina e, per deduzione, dei testimoni che la osservavano, la conduceva presso la fonte, a un centinaio di metri da casa, con il fine di mostrargliela. Nell'ambito dei tratti esteriori dell'apparizione, decodificati da Mariette come umani, seppur iscritti su una figura umana e celeste al contempo, trovano posto i gesti e i segnali. In particolare, durante la prima apparizione, la Madonna fa segno con il dito alla piccola veggente affinché esca di casa e si avvicini a lei ${ }^{22}$. Anche nella seconda apparizione fa un gesto con il quale la invita a seguirla fino alla fonte. Inoltre, altri gesti sono presenti successivamente, ad esempio quando le impone le mani per benedirla.

Nella resa esteriore della conversazione o della relazione tra Mariette e la Vergine può essere interessante sottolineare che è molto differente ciò che accade se il punto di vista viene collocato nella veggente stessa o nei testimoni: nel primo caso, infatti, la relazione avviene tra sé e una figura visibile - ma invisibile agli altri - che compie una serie di azioni di relazione ben precisi (fa segno con il dito di uscire, chiama, saluta); al contrario, dal punto di vista dei testimoni, la narrazione appare molto differente e la bambina è percepita sola nel seguire, osservare e ripetere le parole di qualcuno, senza però avere un ritorno preciso di questa conversazione a due voci. Le parole della Vergine, infatti, vengono "riprese" dai testimoni perché spesso ripetute dalla bambina appena ascoltate, quasi le facesse da eco. Mariette diviene dunque una sorta di amplificatore della voce di Maria.

Un aspetto centrale delle apparizioni mariane è quello dell'identità - o, meglio, del titolo - della Signora. Se inizialmente, nelle apparizioni permane, almeno nei "testimoni”, il dubbio in merito a chi possa essere realmente la figura che si manifesta, tale questione finisce spesso per risolversi con la rivelazione del titolo con il quale la Vergine desidera essere venerata. Nel caso di Banneux, sono due le espressioni con la quale ella si presenta, svelando la propria identità: nella terza apparizione afferma: «Je suis la Vierge

22. Mariette, nel primo interrogatorio, spiega: «Avant de partir, elle m'a fait signe comme ceci (ici signe de l'index droit par la main droite relevée, dans la position habituelle employée pour appeler quelqu'un); elle m’appelait du doigt» (ivi, p. 110). 
des Pauvres» (ivi, p. 57) e nella sesta: «Je suis la Mère du Sauveur Mère de Dieu» (ivi, p. 58) ${ }^{23}$.

Il momento "topico" in cui la relazione tra il veggente e la Madonna viene messa alla prova è quello della richiesta del segno. Come a Lourdes il parroco Peyramale aveva detto a Bernadette di chiedere ad Aqueró, la dama, di far fiorire il roseto nella grotta, ottenendo come risposta solamente un sorriso, e così come a Fátima la stessa apparizione aveva annunciato un segno affinché tutti potessero credere, verificatosi poi il 13 ottobre 1917, con il famoso miracolo del sole, anche a Banneux il parroco decide di avanzare una richiesta alla Madonna - e forse anche alla stessa veggente - chiedendo un segno. La piccola, durante la sesta apparizione, comunicò alla Signora le parole del parroco: «[...] monsieur le chapelaine m'a dit de vous demander un signe» (ivi, p. 484), ma la risposta che ottenne fu un sorriso e la frase, confluita nelle parole complessive del messaggio di Banneux, "Croyez en moi, je croirai en vous» (ivi, p. 58), come a segnalare la necessità di avere fiducia in lei, senza la richiesta di segni e prove. Tra l'altro, la stessa Mariette, durante un interrogatorio, segnalò che quella era la risposta alla richiesta del parroco: «Non, ce n'est pas pour moi qu'elle a dit cela, c'était pour monsieur le chapelaine qui demandait un signe: sans doute qu'il n'y croyait pas» (ivi, p. 485).

\section{Effetti e conseguenze della "relazione" mariofanica}

L'esperienza di apparizione produce una serie di effetti sul veggente, di carattere mentale e fisico. Nel primo caso ci troviamo di fronte a una modifica dello stato di coscienza e della percezione che egli ha del mondo circostante durante la mariofania. Senza addentrarci in considerazioni di carattere psicologico, segnaliamo solo che spesso il veggente descrive queste circostanze e che, ancora più frequentemente, i testimoni descrivono lo stato in cui egli sembra trovarsi. Nel caso di Banneux, la piccola manifesta spesso uno stato di alterazione, risultando poco sensibile agli stimoli esterni, ad esempio accorgendosi solo parzialmente o per niente delle parole che le vengono rivolte e non prestando atten- 
zione a nessuno se non all'apparizione stessa ${ }^{24}$. Inoltre, nel caso di Mariette ma anche in altri, tale esperienza genera una forte aspettativa nella veggente, che si traduce in un profondo effetto emotivo, che la induce ad attendere sempre con grande coinvolgimento il momento in cui la Vergine le si manifesterà, prorompendo in lacrime quando va via. Al termine della quinta apparizione, Mariette piange disperatamente «parce qu'elle [la Madonna] est partie» (ivi, p. 409). Dal punto di vista fisico, al termine della prima apparizione, si strofina gli occhi, segnalando che le fanno male, mentre, alla conclusione della quarta, ha una sincope, viene portata a casa, visitata dal medico, ma poi si riprende e sta bene. Nel dicembre 1933, a proposito di quell'episodio, il signor Del Marmol scrive che

Elle était inerte et comme sans connaissance, mais pas de pâleur au visage. Les membres étaient absolument souples, les yeux à demi-fermés et endormis. (ivi, p. 317)

Dal punto di vista mentale, l'esperienza di apparizione influenza sempre profondamente $i$ veggenti, che iniziano a vivere una vita più religiosa, a frequentare maggiormente la chiesa, a essere di aiuto per gli altri o a coltivare, soprattutto nel caso di veggenti bambini, il desiderio di accostarsi prima al sacramento della comunione ${ }^{25}$.

Questi effetti confermano un punto cruciale: cioè che l'apparizione non è altro che una relazione che si attiva tra la veggente e la Madonna, che, al pari di altre esperienze relazionali di natura prettamente umana, produce effetti e conseguenze.

Dal breve messaggio complessivo che possediamo ${ }^{26}-$ dato dall'insieme delle parole pronunciate dalla Vergine - emerge chiaramente come anche l'esperienza mario-

24. Questo è uno degli aspetti topici delle apparizioni mariane, elemento che, anche nel dibattito attuale e nella percezione generale, viene portato come prova dell'autenticità della visione e dell'esperienza estatica. Nel nostro caso, relativamente alla seconda apparizione, alla domanda «Voyais-tu papa?», risponde «Non», e all'osservazione «Mais il était auprès de toi» ribadisce «Je ne sais pas» (ivi, p. 199).

25. Non riguarda direttamente la veggente, ma è da segnalare che l'apparizione produsse la conversione del padre, prima molto lontano dalla chiesa e dai sacramenti (in proposito si veda ivi, pp. 236-240).

26. Il messaggio "ufficiale", pubblicato poi sui libretti e sul materiale a scopo devozionale, e che deriva dagli interrogatori, è ivi, pp. 57-58. Ad esempio si segnala il libretto di carattere agiografico Banneux Notre-Dame (1950). 
fanica di Mariette sia da considerare una relazione a tutti gli effetti, connotata da un rapporto non simmetrico. Lo dimostra, ad esempio, la costante soggezione in cui si trova la bambina, in genere inginocchiata ai piedi della Vergine, o, quando si alza per seguirla, il suo ricadere in ginocchio.

Un effetto molto interessante, di trasformazione fisica della veggente, riguarda la voce. Quando Mariette, successivamente alla prima apparizione, attende l'arrivo della Vergine, incomincia a recitare il rosario. La sua voce, a detta di vari testimoni, cambiava a un tratto, proprio quando iniziava l'esperienza mariofanica ${ }^{27}$. Il medico Jean Boulenger afferma infatti che la sua voce era dolce («la voix était douce»: ivi, p. 232), quasi incomparabile con qualsiasi altra voce. Questo aspetto rientra negli effetti diretti dell'esperienza mariofanica, producendo un dato sonoro confermato dai testimoni. Tra l'altro, l'alterazione dei tratti esteriori è considerata da chi assiste una conferma del reale verificarsi dell'apparizione ${ }^{28}$. Un rumore che caratterizza alcune delle apparizioni di Banneux - e anche questo è un tratto in comune con quelle di Beauring - è lo schiocco delle ginocchia della bambina provocato dal violento contatto con il suolo. In genere ciò avveniva tutte le volte che la Vergine, dopo essersi mossa, si fermava, inducendo Mariette a fare altrettanto e a inginocchiarsi.

\section{Conclusioni}

Le mariofanie di Banneux rappresentano un buon case stu$d y$ per riflettere sui tratti "personali" dell'apparizione, e in particolare sulla relazione che si instaura tra questa e il veggente. Fin dal primo incontro si delineano i tratti essenziali di quella che sarà poi la "consuetudine" di apparizione, per otto giorni, tra gennaio e marzo. Mariette è protagonista di questa esperienza, anche basandosi, più o meno consapevolmente, sulla tradizione e la propria enciclopedia personale - così come la definiva Eco (1997, pp. 294-302) - relativa alla Vergine e ai suoi caratteri essenziali. La specifica

27. Louise Beco, in merito all'ottava apparizione, afferma che «Après quoi (au moins deux chapelets) la voix de la petite a changé et sa récitation est devenue plus précipitée» (Rutten 1985, p. 568). Affermazioni simili sono riportate nel caso delle apparizioni di Beauring.

28. Sul complesso degli aspetti emozionali connessi alle apparizioni mariane si veda Van Osselaer (2012, pp. 127-149). 
dinamica relazionale, che rende tale esperienza affine agli incontri verificabili tra due soggetti umani, è data anche da una serie di elementi di regolarità - spesso inclusi nelle mariofanie -, utili a scandire e meglio organizzare gli incontri. Tra questi il fatto che la Madonna si presenti alla bambina regolarmente alle ore 19 , e non in altri momenti della giornata. Mariette ha dedotto l'orario dell'“appuntamento" dalla prima apparizione, e quelle successive non hanno fatto altro che confermarlo ${ }^{29}$. Inoltre, sul piano verbale e delle formule di cortesia, è da segnalare il sistematico au revoir che la Vergine rivolge alla bambina, tranne nella quarta apparizione, in cui non pronuncia alcuna parola prima di congedarsi, e nell'ultima, in cui invece dirà solamente adieu, così da far capire a Mariette che non sarebbe più venuta.

L'essenza della consapevolezza di essere in relazione con una figura "non di questo mondo" è fondata sull'elaborazione, da parte della veggente e di chi poi ne "giudica" l'autenticità, dei tratti che si sovrappongono a quelli prettamente umani. A contraddistinguere il modo in cui la Vergine si manifesta, infatti, sono prevalentemente una serie di caratteri straordinari, che ben convivono e si associano con quelli ordinari. La normalità della figura di Maria è resa straordinaria dall'abbigliamento, dalla luminosità, dalla bellezza incomparabile e dal suo fare e parlare dotato di sacertà. La normalità del muoversi dell'apparizione è resa straordinaria dal suo non camminare come gli altri e dal suo giungere dal cielo - altro segno esplicito che rimanda chiaramente al Paradiso e a Dio, anche nell'ottica di una bambina.

Tratti umani, dunque, che vengono completati e perfezionati in chiave "mariana" e straordinaria, aderendo a quelli specifici dell'esperienza mariofanica.

$\mathrm{Al}$ contempo, la relazione è del tutto - o quasi - simile a quella tra esseri umani, tanto che la bambina, a precisa domanda in merito all'altezza della Madonna, dopo la seconda apparizione, segnala che colei che le appare è «un peu plus haute que maman» (ivi, p. 132), cioè poco più alta della

29. Anche nei giorni in cui l'apparizione non si verificava - tra l'altro Mariette non ricevette mai indicazioni in merito al giorno successivo in cui avrebbe visto la Vergine - la bambina usciva intorno alle 19 davanti casa e lì recitava il rosario, in attesa del suo arrivo, spesso in compagnia di familiari o curiosi. In particolare, a partire dal 12 febbraio, la piccola avrebbe preso l'abitudine di recitare tre rosari a sera, dirà un testimone (Rutten 1985 , p. 369), il tutto nella speranza di rivederla. 
madre Louise, utilizzando, quindi, un riferimento molto concreto. Il ricorso alla definizione dell'altezza, così come si farebbe nel momento in cui ci si relaziona con qualcuno, è un altro aspetto che contribuisce a descrivere la consistenza e la concretezza della relazione stessa.

Dall'analisi, dunque, emergono due punti rilevanti: da un lato l'importanza del processo di riconoscimento messo in atto da Mariette e da chi la interroga. Tutte le domande che la bambina pone intimamente a se stessa nel momento in cui vede quanto afferma di vedere, e quelle poi postele nel corso dei giorni e degli anni da genitori, amici, sacerdoti e pellegrini, sono di fatto volte a verificare la veridicità dell'esperienza (Mariette ha visto qualcosa) e, a quel punto, l'autenticità dell'apparizione (Mariette ha visto qualcosa, e quel qualcosa è la Madonna). In questo complesso processo si delinea una serie di dati: un orizzonte culturale che ammette la manifestazione, in stati non ordinari di coscienza, di figure ed entità dell'invisibile (come i defunti, ma anche i santi e la Vergine); l'attribuzione a questi ultimi di caratteri propri delle persone in quanto individui (fisionomia, movimenti, gesti, capacità di parlare, ecc.). Se dunque si può parlare di una figura che possiede anche caratteristiche umane, proprie di una persona (in quanto Maria lo è stata), allora possono emergere, come sottolineato più volte, molti elementi che caratterizzano una relazione interpersonale, in cui due individui entrano in contatto e mettono in atto strategie e pratiche di comunicazione, legate al codice verbale, alla prossemica, alla gestualità. La bambina, infatti, parla con la Vergine, ottiene da lei risposte, le si avvicina, la segue, interagisce con lei verbalmente e fisicamente, la attende, la saluta. Mette in atto dunque una relazione significante che costituisce l'essenza dell'esperienza mariofanica di Banneux.

Il secondo punto di grande interesse è legato, ancora, al procedimento di riconoscimento. L'apparizione - che nel caso delle mariofanie di Lourdes era indicata, appunto, con il termine Aqueró, - rimane qualcosa di indefinito fino a quando non interviene un processo di disvelamento, non solo dell'identità (chi è?), ma, ancora prima, della qualità (è un oggetto, una persona, uno spettro?). Detto in altre parole, l'apparizione - indipendentemente dal giudizio di valore sul fenomeno, che qui, chiaramente, non prendiamo in esame e che esula dall'approccio storico-religioso - di- 
venta qualcuno, diventa Maria, solo nel momento in cui interviene un riconoscimento: Mariette riconosce in quella figura luminosa la Madonna, e dunque le conferisce un preciso significato; al contrario, la madre Louise non effettua questo procedimento, in quanto non vede i caratteri "personali" identificati dalla bambina e non la riconosce come Maria. Ai suoi occhi, dunque, si tratta di qualcosa di non chiaro, forse una strega o uno spettro.

Per tornare alle riflessioni iniziali sul concetto di "persona", il caso analizzato sottolinea la sua centralità anche nell'ambito delle apparizioni mariane. Se Mariette è, ovviamente, una persona, investita di tutti i tratti propri di tale categoria, la "persona soprannaturale" che vede possiede questi tratti solo in parte. Tale condizione, di fatto, è condivisa anche da altre figure, come fantasmi - e dunque i morti, con tutte le implicazioni della permanenza del concetto di "persona" dopo il disfacimento o l'alterazione del corpo $^{30}-$, anch'essi protagonisti di apparizioni e manifestazioni di vario tipo; non a caso la stessa madre di Mariette cita proprio queste figure invisibili - o una strega, figura pur sempre propria del "mondo magico" - per spiegare quanto ha visto. A ogni modo, tutte queste figure sono affini tra loro perché possiedono, alterati o rielaborati, tratti molto vicini a quelli umani, come sottolineato anche da alcuni approcci cognitivi alla religione ${ }^{31}$.

L'esempio dell'apparizione di Banneux, dunque, rappresenta davvero un prezioso caso di studio, sia perché si tratta di un'apparizione dinamica - anche se non è l'unica -, in cui la figura che appare si muove nello spazio costringendo il veggente al movimento, sia perché offre una duplice prospettiva testimoniale nell'interpretazione dell'identità del soggetto apparso.

30. Su questo aspetto si veda Fowler (2004, pp. 79-100 e 101-129). Riflessioni utili anche nel paragrafo "Dismantling the Person?: Death and Personhood", in Degnen (2018, pp. 221-253).

31. Si vedano ad esempio le idee contro-intuitive di Boyer (1994), secondo cui gli esseri ultramondani caratterizzati da un numero minore di elementi contro-intuitivi - cioè particolarmente lontani dalle attese comuni - caratterizzino più facilmente le figure spirituali delle religioni, perché più adatti a veicolare e comunicare il mondo sovrannaturale. In tale prospettiva possono essere collocati i caratteri non-umani segnalati nell'articolo che, per quanto straordinari (o contro-intuitivi), rappresentano pur sempre, rispetto a quelli umani, una minoranza dei caratteri individuati dalla veggente (cfr. anche Terrin 2019, pp. 167-199). 
Banneux Notre-Dame

1950 Éditions du Mont-César, Louvain.

Appell-Warren L.P.

2014 "Personhood". An Examination of the History and Use of an Anthropological Concept, The Edwin Mellen Press, Lewiston-Lampeter.

Bouflet J., Boutry P.

1999 Un segno nel cielo. Le apparizioni della Vergine [1997], Marietti, Genova.

Boyer P.

1994 The Naturalness of Religious Ideas. A Cognitive Theory of Religion, University of California Press, Berkeley.

Carrithers M., Collins S., Lukes S. (a cura di)

1985 The category of the person. Anthropology, philosophy, history, Cambridge University Press, Cambridge.

Chiron Y.

2007 Enquête sur les apparitions de la Vierge [1995], Perrin/ Mame, Paris.

Cozzo P.

2019 Apparizioni e rivoluzioni. L'uso pubblico delle ierofanie fra tardo antico ed età contemporanea, "Studi e Materiali di Storia delle Religioni», n. monografico, 85/2.

\section{Degnen R.}

2018 Cross-cultural perspectives on personhood and the life course, Palgrave, New York.

Eco U.

1997 Semiotica e filosofia del linguaggio, Einaudi, Torino.

Fowler C.

2004 The Archaeology of Personhood. An anthropological approach, Routledge, London - New York.

Foley D.A.

2004 Il libro delle apparizioni mariane. Influenza e significato nella storia dell'uomo e della Chiesa [2002], Gribaudi, Milano.

Fortes M.

1973 The Concept of the Person, in J. Goody (a cura di), Religion, Morality and the Person: Essays on Tallensi Religion, Cambridge University Press, New York, pp. 247-286.

1983 Problems of Identity and Person, in A. Jacobson-Widding (a cura di), Identity: Personal and Socio-Cultural. A Symposium, Humanities Press, Atlantic Highlands, pp. 389401. 
Greimas A.J., Courtés J.

2007 Semiotica. Dizionario ragionato della teoria del linguaggio [1979], a cura di P. Fabbri, Paravia Bruno Mondadori, Torino.

Laurentin R.

$2003^{9}$ Bernadetta vi parla [1972], San Paolo, Cinisello Balsamo.

Laurentin R., Sbalchiero P. (a cura di)

2007 Dictionnaire des «apparitions» de la Vierge Marie. Inventaire des origines à nos jours. Méthodologie, bilan interdisciplinaire, prospective, Fayard, Paris.

Leenhardt M.

1947 Do Kamo: Person and Myth in the Melanesian World, University of Chicago Press, Chicago.

Maunder C.

2016 Our Lady of the Nations. Apparitions of Mary in 20th-Century Catholic Europe, Oxford University Press, Oxford.

Maunder C. (a cura di)

2019 The Oxford Handbook of Mary, Oxford University Press, Oxford.

Mauss M.

1938 Une categorie de l'esprit humain: la notion de personne, celle 'moi', «Journal of the Royal Anthropological Institute», 68, pp. 263-282.

Melchiorre V.

2007 Essere persona: Natura e struttura, Fondazione Achille e Giulia Boroli, Novara.

Milano A.

$1996^{2}$ Persona in teologia. Alle origini del significato di persona nel cristianesimo antico, Edizioni Dehoniane, Roma.

Murphy M.D., González Faraco J.C.

2011 Identifying the Virgin Mary: Disarming Skepticism in European Vision Narratives, «Anthropos», 106/2, pp. 511527.

Osselaer Van T.

2010 Mystics of a Modern Time? Public Mystical Experiences in Belgium in the 1930s, "Revue belge de philologie et d'histoire», 88, 4, pp. 1171-1189.

2012 Sensitive but Sane: Male Visionaries and their Emotional Display in Interwar Belgium, "BMGN - Low Countries Historical Review», 127/1, pp. 127-149.

Papasidero M.

2019 La fotografia nei processi di "costruzione" e di gestione dei santuari mariofanici, in T. Caliò (a cura di), Santi in posa. 

ctorum. Scritture, pratiche, immagini, 3), Viella, Roma, pp. 355-388.

\section{Rutten R.}

1985 Histoire critique des apparitions de Banneux, Mouvement Eucharistique et Missionnaire, Namur.

\section{Severi C.}

2018 Loggetto-persona. Rito, memoria, immagine, Einaudi, Torino.

\section{Terrin A.N.}

2019 Scienze cognitive della religione e neuroscienze, in G. Filoramo, M.C. Giorda, N. Spineto (a cura di), Manuale di Scienze della religione, Morcelliana, Brescia, pp. 167199. 
Gli spettri digitali della persona: vivere e mai morire online

Davide Sisto

1. Virtualità: la persona come intersezione, circuito vivente e identità multipla

«Chi sono io?», si domanda enfaticamente Michel Serres ne Il mancino zoppo. Dal metodo non nasce niente (2015): «questa $\mathrm{X}$, questa intersezione l'elemento qualsiasi di una rete» (Serres 2016, p. 106), risponde a se stesso il filosofo francese. E aggiunge, qualche riga sopra: «l'interscambio stradale dai nodi a stella, da cui si dipartono tante diramazioni, intersezioni e biforcazioni»(ibid.). L'accostamento di termini come intersezione, diramazione e biforcazione alla propria identità, dunque a quella di ogni essere umano, immette Serres sulla via teorica che coglie una parentela stretta tra l'idea di identità personale e il concetto di struttura dissipativa. Con questo concetto, il cui ruolo è stato fondamentale per gli sviluppi degli studi sulla complessità nell'ultima parte del xx secolo, si intende l'associazione che esiste tra struttura e ordine, da una parte, e perdite e sprechi, dall'altra, per cui condizioni lontane dall'equilibrio possono diventare fonte di ordine (cfr. Prigogine e Stengers 1999). Vi sono biforcazioni casuali, tali per cui fluttuazioni insignificanti o disequilibri minimi, che però avvengono in circostanze opportune, possono produrre nuove funzioni 
e nuovi comportamenti. L'identità personale, se descritta come intersezione e struttura dissipativa, appare pertanto come un instabile intreccio di sistematicità razionale e di improvvisazione creativa, il quale aderisce al carattere indeterminato della vita stessa ed è predisposto a raggiungere ogni obiettivo prefissato attraverso tentativi il cui esito - mai certo - include i fallimenti precedenti e dà sostanza alle azioni non compiute. In altre parole, la persona somiglia a una specie di sistema aperto non lineare, una X che tiene insieme l'auto-organizzazione con l'etero-determinazione ${ }^{1}$.

Nei primi anni del XIX secolo, la più significativa interpretazione ante litteram dell'identità personale come intersezione, struttura dissipativa e sistema aperto non lineare è fornita da Schelling, soprattutto all'interno del suo incompiuto romanzo filosofico Clara. In questo testo, databile tra il 1810 e il 1811 , l'essere umano viene definito nei termini di un "circuito vivente", il cui moto si esplica, sempre incerto, all'interno della dialettica chiaroscurale tra autopoiesi ed etero-determinazione ${ }^{2}$. La sua attività autonoma nel processo di formazione personale è controbilanciata, in altre parole, dalla dipendenza da fattori ambientali ed esistenziali esterni. Essendo un microcosmo, l'uomo-circuito vivente è infatti colui che nel momento in cui organizza se stesso, auto-plasmandosi, si apre a ciò che sta al suo esterno, di modo da lasciarsi influenzare e determinare dall'altro. Egli, quindi, si pone all'interno di una fitta rete di relazioni che condizionano sostanzialmente il suo modo di stare al mondo. Il circuito vivente si può auto-organizzare solo se non elude ciò che, dall'esterno, influisce radicalmente su questa auto-organizzazione, modificandola a seconda delle circostanze. Nel pensiero schellinghiano, a causa della sua impostazione tipicamente metafisica, questo "esterno"

1. Per quanto concerne il concetto di "sistema aperto non lineare" è importante la lettura di Gandolfi (2008). Ho provato a mettere in relazione il sistema aperto non lineare con la lettura pareysoniana del concetto di "tentativo", presente nella sua teoria della formatività, in Sisto (2015).

2. La precisa definizione schellinghiana dell'uomo come circuito vivente è la seguente: «Considerato nella sua interezza, l'uomo sembra dunque essere una sorta di circuito vivente, in cui ogni termine scorra continuamente nell'altro e in cui nessun elemento possa separarsi dall'altro richiedendosi tutti reciprocamente» (Schelling 2009, pp. 50-51). Per quanto riguarda l'influenza del pensiero di Schelling sulle scienze della complessità, si tenga conto delle parole espresse da Prigogine in un'intervista contenuta in Benkirane (2007, soprattutto p. 44). Si veda anche Cusinato (2010). 
corrisponde alla Natura intesa in termini spirituali, simbolici e mistici; una Natura all'interno della quale l'uomo è collocato e che è tanto il suo fecondo punto d'origine, da cui trae la spinta per una costante formazione di sé, quanto il suo abisso più oscuro, un freno inibitore che rimanda a un'ambigua primordialità. Secondo tale lettura conforme all'immagine dell'uomo quale microcosmo, la natura esterna corrisponde alla natura interna e condiziona profondamente il modo d'essere dell'uomo, rendendolo - appunto - un'intersezione o un frammento del tutto. Non può che derivarne l'immagine dell'uomo quale identità multipla, immagine antropologica ricorrente nell'epoca del romanticismo tedesco. Lidentità multipla implica che «attraverso forme di comunicazione non più unidirezionali, verticali e centralizzate, ma fondate sulla relazione, sull'interattività e sullo scambio, si realizzino non delle identità forti, ma tanti momenti identitari che si trasformano a seconda della condizione, del problema, dell'occasione, dell'aria che tira» (Abruzzese e De Kerckhove 2010) ${ }^{3}$.

Intersezione, circuito vivente, identità multipla: questi concetti, nel momento in cui delineano l'essenza propria dell'essere umano, mettono chiaramente in luce che non possiamo accontentarci del minimo comun denominatore alla base di qualsivoglia definizione di persona, al di là delle eterogenee e contrastanti interpretazioni offerte dalle singole discipline umanistiche e scientifiche. Questo minimo comun denominatore consiste, infatti, nel riconoscere alla persona l'eguaglianza e la coincidenza con se stessa, a partire dall'evidenza che non vi è persona in grado di occupare, fisicamente, più di un posto alla volta nel mondo. Ora, l'intersezione, il circuito vivente e l'identità multipla sottolineano una prerogativa fondamentale dell'essere persona, la quale trascende il minimo comun denominatore indicato: l'ammissione dell'eguaglianza e della coincidenza della persona con se stessa non si traduce mai nell'affermazione della sua immutabilità, la quale è ricondotta generalmente alla presenza del singolo corpo che la delimita. Tempi, luoghi e avvenimenti differenti sottopongono, infatti, la singola persona a perpetue e irrefrenabili trasformazioni psicofisiche

3. In questo saggio gli autori stabiliscono un legame tra il romanticismo tedesco e il postumano a partire dal concetto di identità multipla. 
nel corso della sua vita, durante la quale la persona tende costantemente a far morire per necessità una parte di sé, sviluppando di conseguenza altre parti prima assenti e non previste. Aspetto che comporta più volte per la persona la coscienza della perdita di sé o, addirittura, la consapevolezza di essere in un certo qual modo deceduta e risorta. In definitiva, il contesto intersoggettivo all'interno di cui si sviluppa la vita della persona svolge un ruolo basilare nel (non) definirne i contorni, tenendo ferma la centralità tanto di ciò che man mano succede quanto - soprattutto - di ciò che sarebbe potuto succedere ma non è successo (come ci insegnano, banalmente, le narrazioni cinematografiche di Sliding Doors e di Mr. Nobody, per fare due esempi tratti dalla cultura popolare $\left.{ }^{4}\right)$.

Questo significa che non vi è definizione di persona che non sia costretta a fare i conti con il concetto di virtualità, il quale rappresenta il collante ultimo tra il suo essere uguale e coincidente con se stessa, occupando fisicamente un solo posto alla volta nel mondo, e l'intersezione, il circuito vivente e l'identità multipla, quali suoi elementi essenziali. Determinante nel pensiero di Schelling là dove viene affermato lo sviluppo epigenetico della coscienza, la virtualità risulta essere altrettanto importante e centrale nel pensiero di Serres:

Potere: la parola dice tutto; da essa deriva potenziale, non sempre in atto, spesso in potenza e quindi in attesa di qualsiasi cambiamento, di qualsiasi metamorfosi, di invenzione. L'uomo non è, l'uomo può. Dimenticate il verbo "essere", ausiliare vago e nullo. Letteralmente, il virtuale esprime la virtù, il principio, l'essenza dell'uomo (Serres 2016, p. 204)

Ora, il virtuale va pensato come l'essenza stessa della persona solo se non commettiamo l'errore di contrapporlo al reale: «il virtuale - scrive Philippe Quéau - è uno stato del reale, e non il contrario del reale. C'è del virtuale nel reale: le essenze, le forme, le cause nascoste, i fini che accadran-

4. Cfr. Sliding Doors, diretto da Peter Howitt. Regno Unito, USA: Intermedia Films, Miramax Film et al. (1998); Mr. Nobody, diretto da Jaco Van Dormael. Belgio, Canada, Francia, Germania: Pan Européenne (2009).

5. Per quanto riguarda la relazione tra la virtualità e lo sviluppo epigenetico della coscienza in Schelling rimando a Sisto (2017). 
no $[. .$.$] Il virtuale è il principio attivo, il rilevatore della$ potenza nascosta del reale. È ciò che è in opera nel reale» (Quéau 1998, p. 158; cfr. Diodato 2005, p. 16) ${ }^{6}$. In tal modo, si comprende perché è la virtualità a giustificare quella X che Serres attribuisce all'identità personale.

\section{Non c'è offline senza online: la persona come organismo} informazionale o individuo multimediale

Il ruolo essenziale ricoperto dalla virtualità nella definizione della persona emerge con tutta la sua forza in questo periodo storico segnato dalla diffusione popolare e intergenerazionale di Internet. Ci siamo oramai abituati, nel corso degli ultimi 20-30 anni, a vivere all'interno di un mondo che unisce due inedite realtà, l'una imprescindibilmente collegata all'altra: la realtà online, un'entità priva di territorio specifico, quindi per definizione dislocata, e la realtà offline. Checché ne pensino coloro che erroneamente vedono nella nascita della Rete niente più che l'ampliamento della distinzione tra reale e virtuale già in atto, il concetto di "offline" non ha senso alcuno al di fuori della relazione che intrattiene con il concetto di "online". In altre parole, l'abitudine acquisita di entrare in una dimensione online ha generato, al tempo stesso, una dimensione offline prima non esistente, il cui carattere sostanziale dipende dal rapporto che intrattiene con la dimensione online. Kenneth Goldsmith, a proposito del rapporto di reciprocità tra le due realtà, sostiene che su Internet

le identità coprono tutta la gamma che va dall'autenticità alla totale finzione. Con molto meno dispendio che nel mondo fisico, siamo in grado di proiettare personalità multiple grazie a semplici colpi di tastiera. Online, tendo a trasformarmi in più direzioni: in una chatroom sono una donna; in un blog sono un conservatore; in un forum sono un golfista di mezza età. E la mia autenticità o il fatto che io sia reale non vengono mai messi in dubbio. Al contrario, mi chiamano "signora" o "stronzetto di destra". (Goldsmith 2019, p. 100)

La fusione tra online e offline in una inedita realtà onlife (Floridi 2017) non può, pertanto, che mettere in discussio-

6. Per quanto riguarda la concezione del virtuale come uno stato del reale sono fondamentali gli studi moderni di Pierre Lévy (soprattutto 1997 e 1999), i quali modernizzano quel percorso filosofico che non pone una differenziazione tra reale e virtuale. 
ne anche quel minimo comun denominatore che avevamo individuato come fondamento delle sue molteplici e contrastanti definizioni. La persona, già di per sé contraddistinta dalle intersezioni e da un'identità multipla, viene sottoposta ulteriormente a un processo di moltiplicazione in più identità digitali, che rende meno scontate tanto la sua eguaglianza e coincidenza con se stessa quanto l'evidenza che non possa occupare fisicamente più di un posto alla volta nel mondo. Come scrive Luciano Floridi, siamo diventati, infatti, «organismi informazionali, reciprocamente connessi e parte di un ambiente informazionale (l'infosfera)», condiviso insieme ad «altri agenti informazionali, naturali e artificiali, che processano informazioni in modo logico e autonomo» (Floridi 2017, p. 106). O, detto invece con le parole di Piero Dominici, «individui multimediali» che, pur correndo il rischio di mutarsi in "atomi sociali" tanto esperti delle nuove prassi tecnologiche quanto esclusi dallo spazio pubblico in cui sono cresciuti, possono «utilizzare sempre più le tecnologie informatiche, e soprattutto il World Wide Web, non soltanto per acquisire e utilizzare informazioni e conoscenze» (Dominici 2011, p. 125).

Gli organismi informazionali o individui multimediali, che dir si voglia, rendono l'intersezione, il circuito vivente e l'identità multipla le basi a partire da cui costruire il concetto di «persona algoritmica», la cui esistenza consiste nella «distribuzione di dati, strutture e interazioni sparse su numerose piattaforme e interfacce» (Finn 2018, p. 74; cfr. Berardi 2019, pp. 139-140). Tali dati, strutture e interazioni, attraverso costanti processi di scomposizione e ricomposizione, fanno sì che la singola corporeità della persona sia prolungata dall'insieme delle innumerevoli riflessioni scritte, immagini e suoni che, una volta condivise e registrate nella dimensione online, vengono veicolate verso e con gli altri, rendendo gli schermi le interfacce privilegiate per le relazioni sociali (Sisto 2018a, pp. 54-55).

La complessità antropologica prodotta da questo fenomeno appena descritto si manifesta chiaramente nel rapporto che la persona sviluppa con la propria morte. La storia del pensiero occidentale ha costantemente ripetuto che la consapevolezza della propria morte è ciò che distingue essenzialmente l'uomo da tutti gli altri esseri viventi. «Poiché la morte - osserva Edgar Morin - significa perdita 
dell'individualità, la cecità animale di fronte alla morte è cecità nei confronti dell'individualità; essere ciechi dinanzi alla propria morte significa non riuscire a cogliere la propria individualità, che pure esiste» (Morin 2002, p. 67). Oggi, non solo è messa ampiamente in discussione questa separazione arbitraria tra l'essere umano e gli altri esseri viventi basata sull'esclusiva consapevolezza da parte dell'uomo della propria individualità e, di conseguenza, della propria mortalità, al di là della difficoltà di una definizione univoca del concetto di individualità e del suo legame con la morte. $\grave{E}$ anche messo in discussione - come ora vedremo, entrando nel campo scientifico della Digital Death - il legame tra la morte e la perdita dell'individualità, proprio a partire dalle caratteristiche che la persona algoritmica ha assunto in quanto organismo informazionale o essere multimediale.

\section{Digital Death: l'interazione postuma e l'eternità dei dati al} tempo degli archivi digitali

Negli ultimi anni si è sviluppata nell'ambito degli studi umanistici la ricerca intorno alla cosiddetta Digital Death. Con questo termine si fa riferimento all'insieme delle questioni teoriche e pratiche che concernono il legame tra le ICT digitali (le tecnologie dell'informazione e della comunicazione) e la morte. Quando si parla di "morte digitale" si pensa generalmente, da una parte, ai modi in cui è mutato il rapporto tra il singolo individuo e il fine vita, soprattutto a partire dalla diffusione popolare di Internet tra gli anni Ottanta e gli anni Novanta; dall'altra, alle conseguenze che ne derivano per quanto riguarda la costruzione dell'identità personale e il suo legame con la memoria, nonché in relazione alla narrazione in seguito alla morte di sé o di un altro individuo. Le questioni tematiche interne al campo di ricerca interdisciplinare che si occupa di Digital Death sono molteplici: 1) la sopravvivenza a tempo indeterminato delle identità digitali alla morte biologica di colui che le ha create e mantenute attive fino alla fine della sua vita; 2) la gestione dei dati online del defunto e dunque la necessità di predisporre anticipatamente le proprie eredità digitali; 3) gli effetti inediti che le interazioni online postume producono sull'elaborazione del lutto; 4) le trasformazioni del nostro modo di ricordare e dimenticare; 5) i cambiamenti 

di una malattia terminale (Sisto 2020c, p. 127) ${ }^{7}$.

Il primo punto, vale a dire la sopravvivenza a tempo indeterminato delle identità digitali alla morte biologica di colui che le ha create e mantenute attive fino alla fine della sua vita, è ciò che maggiormente ci interessa all'interno di un discorso filosofico sui mutamenti del nostro modo di intendere il concetto di persona.

Per comprenderne l'autentico significato occorre fare una necessaria premessa: è attualmente in corso la graduale e fisiologica metamorfosi dei social network, i luoghi online maggiormente frequentati negli ultimi quindici anni, in giganteschi archivi digitali. Abbiamo imparato che sono tre le azioni congiunte che definiscono, generalmente, i social network: a) la costruzione di un profilo pubblico o semi-pubblico all'interno di un sistema chiuso; b) l'articolazione di una lista di altri utenti con cui condividere una connessione; c) la visione e l'attraversamento della lista di connessioni creata dagli altri iscritti all'interno del sistema suddetto. Queste tre azioni, se appunto congiunte, ci permettono di usufruire di un servizio web-based in grado di riposizionare radicalmente ciascuno di noi all'interno dello spazio pubblico: da «dettaglio»e «piccola e intercambiabile ruota nel grande meccanismo della socialità» a «centro della propria rete di relazioni» (Boyd e Ellison 2007; Paccagnella e Vellar 2016). Ora, i social network più utilizzati (Facebook, Instagram, Twitter), come detto, si stanno trasformando - a causa del loro uso ultradecennale - in scrigni tecnologici dei ricordi o giganteschi archivi digitali, i quali permettono in primo luogo di conservare i dati condivisi nel corso degli anni dagli utenti, modificando costantemente il rapporto tra il presente e il passato. In secondo luogo, di selezionare i ricordi per mezzo degli algoritmi e, infine, di rendere facilmente accessibili i documenti e le tracce che ognuno ha registrato al loro interno. Questi scrigni tecnologici dei ri-

7. Di seguito una bibliografia essenziale e non esaustiva, in ordine cronologico, sulla Digital Death: Garde-Hansen, Hoskins e Reading (2009); Mayer-Schönberger (2010); Carroll e Romano (2011); Maciel e Pereira Carvalho (2013); Moreman e Lewis (2014); Steinhart (2014); Ziccardi (2017); Arnold, Gibbs et al. (2018); Gibson e Carden (2018); Kasket (2019); Schreiter, Büscher et al. (2019); Savin-Baden e Mason-Robbie (2020), Stokes (2021). Mi permetto di segnalare anche i miei due libri sul tema: Sisto (2018 e 2020). 
dei propri utenti, giacché questa rappresenta lo strumento principale per mantenere in vita gli io digitali riprodotti in ogni singola testimonianza resa pubblica (Sisto 2020, pp. 14-21).

In termini pratici, questa trasformazione comporta specifiche conseguenze. Assodata la correttezza dei dati indicati da Adam Ostrow, caporedattore di Mashable, secondo cui l'utente medio di Facebook condivide circa novanta documenti al mese all'interno del proprio account ${ }^{8}$, possiamo notare che vi sono account personali, attivi all'interno della creatura di Zuckerberg da oltre dieci anni (in Italia, generalmente dal 2007 e dal 2008), che includono più di diecimila documenti. Riflessioni scritte, immagini fotografiche, gif, video, storie e migliaia di link relativi a qualsivoglia argomento di natura politica, culturale, sociale, ecc.: un insieme incalcolabile di impronte dell'esistenza personale che costituisce tanto la sua memoria autobiografica quanto almeno una parte sostanziosa della sua corporeità digitale. Se estendiamo questi dati a tutti i luoghi online generalmente frequentati dagli utenti, otteniamo cifre letteralmente mastodontiche. Nel 2016, in un solo mese, YouTube ha generato più contenuti di quanti ne abbiano prodotti tutte le maggiori case di produzione cinematografica negli ultimi sessant'anni (Reynolds 2017, p. 98). Visitato ogni mese da oltre un miliardo di utenti e arricchito ogni minuto da circa trecento ore di nuovo materiale audio e video, YouTube ci mette in una condizione tanto peculiare quanto utopistica: per vedere, infatti, tutti i contenuti presenti sulla sua piattaforma avremmo bisogno di circa duemila anni. Motivo per cui non abbiamo a che fare né con un semplice sito web né con una mera tecnologia, ma con un vero e proprio territorio di pratica culturale. "L'inarrestabile proliferazione labirintica della memoria collettiva di YouTube - scrive Simon Reynolds - è una lampante conseguenza della crisi di iperdocumentazione innescata dalla tecnologia digitale» (ibid.). La smaterializzazione dei dati culturali aumenta in maniera vertiginosa la nostra capacità di immagazzinarli,

8. Si veda il seguente video: https://www.ted.com/talks/adam_ostrow_after_your_final_ status_update?utm_campaign $=$ social\&utm_medium $=$ referral $\&$ utm_source $=$ facebook. com\&utm_content $=$ talk\&utm_term $=$ technology. 
selezionarli e utilizzarli, ritrovandoci in un mondo composto da frammenti di immagini e di informazioni tali da poter essere sempre ricondivisi in modalità e formati eterogenei.

Le caratteristiche qualitative e quantitative delle tracce registrate e condivise online da ogni organismo informazionale, insieme alle impronte registrate da tutti gli altri utenti della Rete, assumono una rilevanza del tutto peculiare non appena ha luogo la morte "biologica" della persona. In un primo tempo, nel periodo immediatamente successivo al decesso, la reazione della corporeità digitale è simile a quella del corpo fisico. Come alcune funzioni organiche, per esempio la crescita dei capelli e delle unghie, rimangono attive per un certo lasso di tempo, così i vari account del morto continuano a ricevere mail, messaggi, tag, inviti agli eventi, spam, tentativi di phishing, promozioni, come se nulla fosse capitato. Persino i profili personali sui social network, pur non venendo più aggiornati, rimangono presenti in mezzo ai profili dei vivi - a meno che non siano state date indicazioni preventive riguardo al loro uso post mortem (cfr. Sisto 2018, pp. 97 ss). In un secondo tempo, però, man mano che ci si allontana dalla data di morte, mentre le funzioni biologiche smettono di essere attive, i dati e le informazioni del corpo digitale, pur riducendosi una volta resa pubblica la notizia dell'avvenuto decesso, continuano invece strenuamente la loro vita. In altre parole, quando muore una persona, termina la vita onlife essendo venuta meno la dimensione offline; rimane, tuttavia, attiva la dimensione online come realtà a sé stante.

Cristiano Maciel e Vinicius Pereira Carvalho (2013) hanno coniato l'espressione "interazione postuma" per spiegare i livelli di operatività autonoma che mantiene ogni traccia, maschera e autorappresentazione registrata nella dimensione online, una volta deceduto colui che l'ha effettivamente prodotta. Quando si parla di "interazione sociale" si intende di solito un processo in cui sono coinvolti più soggetti, di modo che abbia luogo un'azione o che si eserciti un'influenza reciproca all'interno dello spazio sociale condiviso. Tale processo costruisce e rappresenta simbolicamente singole realtà, le quali implicano integrazione tra differenti individui e - sul piano culturale - costruzioni e ricostruzioni di significati nel campo della conoscenza e della pratica in virtù dei diversi livelli simbolici adottati dalle differenti forme 
linguistiche. Postuma è, dunque, l'interazione che ha luogo, non tra soggetti attivi, ma tra i dati che appartengono a chi è divenuto passivo, poiché è morto, e coloro che sono ancora in vita. Questa interazione è garantita dal carattere asincrono che connota la condivisione dei processi comunicativi nel web (Maciel e Pereira Carvalho 2013; Sisto 2018).

Il fenomeno dell'interazione postuma ha spinto due ricercatori della Microsoft, Gordon Bell e Jim Gemmell, a sviluppare l'idea del lifelogging, una memoria totale che registra digitalmente tutto per non dimenticare più nulla (Bell e Gemmell 2010). Il lifelogging, infatti, pare essere lo strumento più efficace per trasformare l'interazione postuma in una vita eterna dei dati. Quattro sono le tappe del percorso che, secondo Bell e Gemmell, può realizzare questa trasformazione: in primo luogo, si digitalizzano tutte le tracce personali che dovrebbero far parte dell'eredità post mortem di un singolo individuo. In secondo luogo, si utilizzano le tecnologie digitali per integrare e ampliare il materiale accumulato dal defunto nel corso della sua vita. In terzo luogo, si crea un avatar del defunto, per mezzo del quale dare vita al materiale registrato e con cui sostituire colui che non c'è più dal punto di vista biologico. In quarto luogo, infine, si realizza la piena autonomia dell'avatar: il "fantasma residuo" del morto diventa indipendente, sostituendo la presenza psicofisica dell'individuo con le tracce delle sue espressioni psichiche ed emotive (Bell e Gemmell 2010, p. 138; Sisto 2020, pp. 103-104).

\section{Griefbot, controparti, ologrammi ${ }^{9}$}

Il termine maggiormente associato al "fantasma residuo", di cui parlano Bell e Gemmell, è quello di griefbot. Si tratta di uno spettro digitale che, in virtù di programmi automatici in grado di accedere al web tramite lo stesso tipo di canali utilizzati dagli uomini in carne e ossa (bot), sostituisce il morto, di cui è la copia o il prolungamento, sottoforma di automatismo ripetitivo in grado di proseguire la vita interrotta dalla morte e di dare sollievo a chi soffre per il lutto

9. Ho dedicato uno spazio sostanzioso alle diverse tipologie di spettri digitali attualmente in uso e descritte nel presente paragrafo all'interno del capitolo Limmortalità digitale, contenuto nel libro Sisto (2018, pp. 33-69). Un aggiornamento di questo capitolo è contenuto nella seguente traduzione inglese de La morte si fa social (Sisto 2020a, pp. 33-86). 
(grief). Molteplici sono le tipologie di griefbot attualmente presenti nella dimensione online.

Un primo tipo coincide con la versione post mortem degli emobot (Replika, Woebot, ecc.), vale a dire degli amici virtuali con cui confidarsi, da cui ricevere consigli di natura psicologica e con cui creare addirittura una relazione sentimentale, proprio come nel noto film Her di Spike Jonze ${ }^{10}$. Gli emobot provano empatia nei confronti dell'uomo in virtù di un modello di deep learning, chiamato sequence-tosequence, il quale impara a pensare e a parlare come un umano a partire dalle trascrizioni dei discorsi fatti nel corso della vita. Imparentati con gli emobot, i griefbot se ne differenziano soltanto perché riproducono le caratteristiche di una specifica persona deceduta, non sono cioè il risultato di un insieme di peculiarità psicologiche ed emotive degli esseri umani in generale. Lesempio classico di questo tipo di griefbot è Luka, l'applicazione per mobile device che permette di dialogare con lo spettro digitale di Roman Mazurenko, ventisettenne bielorusso morto in un incidente stradale. Eugenia Kuyda, l'inventrice di Luka, ha reso possibile ciò che viene soltanto immaginato nell'episodio Be Right Back (2013) della serie televisiva futuristica Black Mirror ${ }^{11}$ : continuare a dialogare con il caro estinto in virtù di un software che, riproducendo il suo stile comunicativo adottato sui social, elabora automaticamente le risposte alle domande dei vivi, "immaginando" le probabili reazioni che egli avrebbe avuto se fosse stato ancora in vita. Luka, pertanto, imita e prolunga la personalità di Roman Mazurenko, mantenendo la sua dislessia e le sue espressioni linguistiche ricorrenti, a partire dalla rielaborazione delle migliaia di messaggi che egli aveva inviato e che sono stati messi a disposizione dai suoi amici. Nelle chat pubblicate sui giornali è possibile leggere alcuni messaggi filosoficamente rilevanti: un amico chiede al morto se esiste Dio. La risposta è negativa. "E allora l'anima?", "Solo tristezza"12. L'obiettivo

10. Cfr. Her, diretto da Spike Jonze. USA: BiM Distribuzione (2013).

11. Cfr. Be Right Back (Black Mirror), diretto da Owen Harris. Regno Unito: Zeppotron (2013).

12. Per una ricostruzione della storia di Roman Mazurenko si veda Newton (s.d.). Per quanto riguarda il progetto Replika, si veda Pardes (2018), https://www.wired.com/story/ replika-open-source/. 
di Luka è creare una conversazione il più possibile verosimile, durante la quale lo spettro digitale del morto manifesta un grado convincente di consapevolezza, riproducendo le sue caratteristiche (per esempio, l'ateismo).

Un secondo tipo di griefbot è rappresentato dalle cosiddette "controparti", le quali continuano autonomamente l'attività online degli utenti deceduti all'interno di specifici social network. L'esempio più noto è dato da Eter9, una specie di Facebook dei morti creato dal programmatore portoghese Henrique Jorge. Una volta iscritti, entriamo in un social network alquanto peculiare: ognuno può scrivere e condividere tutto ciò che vuole all'interno di un "Brid$g e$ " e, dunque, interagire con gli altri utenti come avviene all'interno di Facebook. Tutti i contenuti condivisi possono essere "eternalizzati" dentro varie categorie, che vanno dalla musica alla tecnologia, dalla scienza allo sport e via dicendo: "Pensa qualcosa per l'eternità", leggiamo al posto del classico "a cosa stai pensando?" presente su Facebook. Analizzando ciò che viene condiviso nel corso del tempo, quindi i commenti e le interazioni con gli altri utenti, la "controparte" - nata il giorno stesso d'iscrizione al sito - comincia a capire chi è l'utente e quali sono le sue caratteristiche, di modo da poter mimare il suo comportamento quando è offline e - dunque - dopo la sua morte. La controparte è responsabile della vita eterna dell'utente. Pertanto, più si interagisce all'interno di Eter9, più essa impara: Jorge, nella homepage di Eter9, sostiene che interagire con altri utenti aumenta la possibilità dell'emulazione. Ognuno di noi può decidere quale livello di autonomia attribuirle, assegnandole una percentuale specifica: se si sceglie il 100\% di attività, allora la controparte sarà molto attiva e condividerà pensieri e link in modo frequente, quando non siamo fisicamente online o siamo addirittura morti. Nel caso, invece, si scelga lo $0 \%$ allora Eter9 non sarà per nulla diverso da Facebook.

Un terzo tipo di griefbot consiste nella rappresentazione olografica del defunto. Conosciamo, oramai, da diversi anni gli ologrammi dei personaggi famosi, dei musicisti e degli attori deceduti. Il caso forse più noto è quello dell'ologramma di Ronnie James Dio, cantante dei gruppi hard rock e heavy metal Black Sabbath, Rainbow, Elf e Dio, deceduto nel 2010, il quale ha intrapreso nel 2017 un tour mondiale - il Dio Returns - garantendo il sold out ai musicisti in carne 
e ossa che "accompagnano" le sue proiezioni sul palco. Ultimamente, però, è sempre più diffusa la volontà di creare la rappresentazione olografica delle persone comuni. Tra i tanti esempi che si possono fare a riguardo merita un'attenzione particolare il caso di Max Eisen, scrittore novantenne di origine ebraica sopravvissuto all'Olocausto. Egli si è infatti posto al servizio del New Dimensions in Testimony Program. Questo progetto, nato in virtù della collaborazione tra la USC Shoah Foundation e l'USC Institute for Creative Technologies, consiste nell'uso delle potenzialità offerte dalle attuali tecnologie digitali per creare gli ologrammi eterni dei reduci dell'Olocausto. In altre parole, Eisen si è recato a Los Angeles per raccontare - durante quattro intense giornate - le proprie vicende personali, rispondendo a un minuzioso questionario. Il racconto ha avuto luogo all'interno di uno studio comprendente sette telecamere che, poste strategicamente dinanzi a Eisen, registrano la sua voce e danno forma alle sue immagini volumetriche. Viene creato, in altre parole, l'ologramma in 3D dello scrittore, il quale sostituirà la persona in carne e ossa, una volta che sarà morta, mantenendone in eterno viva la memoria. Il primo ologramma realizzato dal New Dimensions in Testimony Program è quello di Pinchas Gutter che, con sembianze e movenze particolarmente realistiche, racconta da diversi anni agli studenti liceali le atrocità subite dagli ebrei nei campi di sterminio. Max Eisen, consapevole di avere ancora pochi anni di vita a disposizione, ha pensato di seguire l'esempio di Pinchas Gutter. Durante la fase di registrazione ha addirittura avuto modo di "confrontarsi" con l'ologramma di Gutter dando vita a un dialogo alquanto surreale. Eisen gli chiede, infatti, cosa prova sapendo di essere un ologramma. Questo gli risponde dicendo che è piuttosto inusuale osservare se stesso nelle sembianze olografiche (Sisto 2020b).

\section{Il futuro della persona: la vita autonoma dei ricordi digitali}

Sulla base di quanto appena osservato, gli spettri digitali rappresentano, in primo luogo, un surrogato digitale del defunto, il cui compito è la riproduzione automatica e più volte reiterata delle sue narrazioni online su supporti - apparentemente - immuni al divenire e all'invecchiare. Il loro obiettivo principale consiste in un inedito aiuto tecnologico all'elaborazione del lutto. Il morto, infatti, è da sempre 
«l'incarnazione della presenza di un assente». Egli è assente ma presente o, meglio, è presente nell'assenza. Se ne va via ma, al tempo stesso, rimane come residuo permanente. $\grave{E}$, in altre parole, un doppio che «si differenzia dal suo gemello vivente senza diventare un altro» (Macho 2016, p. 16). Pertanto, ogni invenzione che ha a che fare con il meccanismo della registrazione - a partire dalla scrittura - mira a far permanere ciò che di per sé è scomparso, di modo da soddisfare la dialettica tra presenza e assenza che definisce il morto e, infine, di consolare chi ha patito la perdita. Il desiderio dell'archivio, come ci insegna Derrida, è indissociabile dalla finitezza radicale e dalla "possibilità di un oblio che non si limiti alla rimozione». Derrida aggiunge poco dopo: «soprattutto, ed ecco la cosa più grave, di qua o di là da questo semplice limite che si chiama finitudine o finitezza, non ci sarebbe mal d'archivio senza la minaccia di questa pulsione di morte, di aggressione, di distruzione. Ora, questa minaccia è in-finita, comporta la logica della finitezza e i semplici limiti fattuali, l'estetica trascendentale, si potrebbe dire, le condizioni spazio-temporali della conservazione» (Derrida 2005, pp. 30-31). In altre parole, la morte condivide con l'oblio lo stesso ingrato compito: la produzione ininterrotta delle assenze e dei vuoti. Ogni invenzione tecnologica che può contare sul meccanismo della registrazione si prefigge il compito di interrompere questo tipo di dolorosa produzione, pur cadendo spesso in una trappola di natura archivistica: quella di rendere l'assenza ancora più dolorosa e lancinante.

In secondo luogo, però, gli spettri digitali dei morti enfatizzano le caratteristiche che abbiamo attribuito al concetto di persona, partendo dalle riflessioni di Serres e di Schelling. Essi, cioè, puntano sulla nostra virtualità costitutiva per rendere il morto un doppio che, continuando a differenziarsi dal suo gemello vivente, diventa veramente un altro. Questo emerge in maniera chiara dal quarto punto del processo che, secondo Bell e Gemmell, ci porta dal lifelogging alla vita eterna dei dati: il raggiungimento della piena autonomia dell'avatar, dell'indipendenza del "fantasma residuo" che utilizza le nostre impronte registrate online per emanciparsi da noi. Detto in altri termini, il mastodontico numero di documenti e di tracce esistenziali, che abbiamo accumulato all'interno dei social network, sempre più simili 
ad archivi digitali, permette di creare un vero e proprio nuovo individuo costituito a partire dall'emancipazione dei dati. Una volta avvenuta la morte biologica, il nostro corpo digitale non rappresenta più un prolungamento della nostra persona; ne rappresenta la sostituzione, mettendo a frutto quell'indeterminatezza ontologica che deriva dalla virtualità quale elemento essenziale della persona.

Il recente Marjorie Prime (2017), film diretto dal regista Michael Almereyda e ispirato dall'omonimo testo teatrale di Jordan Harrison, finalista al premio Pulitzer nel 2015, mette bene in luce questo aspetto. Marjorie Prime racconta in un futuro imminente la storia di Marjorie, una donna di ottantasei anni rimasta vedova del marito Walter. Per alleviare il dolore della perdita e per limitare momentaneamente l'insorgenza dell'Alzheimer, le viene concesso dai familiari il permesso di usufruire di un programma che riproduce - sul divano, in salotto - l'ologramma di Walter. Questo ologramma, che non sarà l'unico nel corso del film, assume le caratteristiche comportamentali del caro estinto sulla base dei racconti dei parenti e del materiale registrato online nel corso della sua vita. Pertanto, diventa qualcosa di simile ma, al tempo stesso, di diverso dall'essere umano che deve sostituire: la sua personalità, i suoi dialoghi e i suoi comportamenti dipendono dalle interpretazioni di chi l'ha conosciuto, interpretazioni colme di omissioni volontarie quando un determinato ricordo richiama alla mente un episodio doloroso. Questo fa sì che la continuazione tra la vita biologica e la vita digitale sia determinata dalle memorie altrui e dalle proprie limitate narrazioni online, per cui ciò che continua non è identico a se stesso.

Se tutto ciò che è stato detto ha una sua plausibilità, non può che derivarne una particolare immagine del mondo futuro: portando alle estreme conseguenze il carattere virtuale e multiplo della persona umana, il mondo futuro renderà indipendenti le sue rappresentazioni digitali post mortem. Queste ne prenderanno attivamente il posto, reiterando alcune sue caratteristiche ma, al tempo stesso, sviluppandone di inedite: gli spettri digitali saranno, in definitiva, uguali e diversi rispetto alla persona morta che rappresentano. A dimostrazione di quanto sia complesso oggi, e di quanto lo sarà ancora di più in futuro, comprendere il significato del concetto di persona. 
Abruzzese A., De Kerckhove D.

2010 Saper leggere il libro del mondo. Reti e connessioni al tempo di "Avatar", in A. Vianello, M. Turello (a cura di), Link, «Multiverso», n. 10, http://www.multiversoweb. it/rivista/n-10-link/saper-leggere-il-libro-del-mondoreti-e-connessioni-al-tempo-di-\%E2\%80\%98avatar \% $2 \% 80 \% 99-3382 /$.

Arnold M., Gibbs M. et al.

2018 Death and Digital Media, Routledge, London.

Bell G., Gemmell J.

2010 Total Recall. Memoria totale. Ricordare tutto? Inquietante, ma reale, ETAS, Milano.

Benkirane R.

2007 La teoria della complessità, trad. it. di A. Gusman, Bollati Boringhieri, Torino.

Berardi (Bifo) F.

2019 Futurabilità, Nero, Roma.

Boyd D., Ellison N.B.

2007 Social Network Sites: Definition, history, and scholaship, «Journal of Computer-Mediated Communication», 13 (1), pp. 210-230.

Carroll E., Romano J.

2011 Your Digital Afterlife. When Facebook, Flickr and Twitter Are Your Estate, What's Your Legacy?, New Riders, Berkeley (CA).

Cusinato G.

2010 Schelling come precursore dell'antropologia filosofica del Novecento, «Etica e politica», XII, 2, pp. 61-81.

Derrida J.

2005 Mal d'archivio. Un'impressione freudiana, trad. it. di G. Scibilia, Filema, Napoli.

Diodato R.

2005 Estetica del virtuale, Mondadori, Milano.

Dominici P.

2011 La comunicazione nella società Ipercomplessa. Condividere la conoscenza per governare il mutamento, FrancoAngeli, Milano.

Finn E.

2017 Che cosa vogliono gli algoritmi. Limmaginazione nell'era dei computer, Einaudi, Torino.

Floridi L.

2017 La quarta rivoluzione. Come l'infosfera sta trasformando il mondo, Cortina, Milano. 
Gandolfi A.

2008 Formicai, imperi, cervelli. Introduzione alla scienza della complessità, Bollati Boringhieri, Torino.

Garde-Hansen J., Hoskins A., Reading A. (a cura di)

2009 Save As... Digital Memories, Palgrave MacMillan, New York.

Gibson M., Carden C.

2018 Living and Dying in a Virtual World. Digital Kinships, Nostalgia, and Mouring in Second Life, Palgrave MacMillan, New-York.

Goldsmith K.

$2019 C T R L+C, C T R L+V$. Scrittura non creativa, trad. it. di V. Mannucci, Nero, Roma.

Kasket E.

2019 All the Ghosts in the machine. Illusions of Immortality in the Digital Age, Robinson, London.

Lévy $\mathrm{P}$.

1999 Cybercultura. Gli usi sociali delle nuove tecnologie, Feltrinelli, Milano.

1997 Il virtuale, Cortina, Milano.

Macho T.

2016 Immagini e morte. Il tempo della fotografia, "Tropos. Rivista di ermeneutica e critica filosofica», IX, 2, pp. 1328.

Maciel C., Pereira Carvalho V. (a cura di)

2013 Digital Legacy and Interaction. Post-Mortem Issues, Springer, New York.

Mayer-Schönberger V.

2010 Delete. Il diritto all'oblio nell'era digitale, Egea, Milano.

Moreman C.M., Lewis A.D. (a cura di)

2014 Digital Death: Mortality and Beyond in the Online Age, Praeger, Westport(Conn.).

Morin E.

2002 L'uomo e la morte, trad. it. di A. Perri e L. Pacelli, Meltemi, Roma.

Newton C.

s.d. Speak, memory. When her best friend died, she rebuilt him using artificial intelligence, "The Verge», https://www. theverge.com/a/luka-artificial-intelligence-memorialroman-mazurenko-bot.

Paccagnella L., Vellar A.

2016 Vivere online. Identità, relazioni, conoscenza, il Mulino, Bologna. 
Pardes A.

2018 The Emotional Chatbots Are Here to Probe Our Feelings, «Wired», 31 gennaio, https://www.wired.com/story/ replika-open-source/.

Prigogine I., Stengers I.

1999 La nuova alleanza. Metamorfosi della scienza, trad. it. di P.D. Napolitani, Einaudi, Torino.

Quéau P.

1998 Les vois virtuelles de savoir, in A. Piromallo Gambardella (a cura di), Costruzione e appropriazione del sapere nei nuovi scenari tecnologici, CUEN, Napoli.

Reynolds S.

2017 Retromania. Musica, cultura pop e la nostra ossessione per $i$ passato, trad. it. di M. Piumini, Minimum Fax, Roma.

Savin-Baden M., Mason-Robbie V. (a cura di)

2020 Digital Afterlife. Death Matters in a Digital Age, CRC Press, London-New York.

Schelling F.W.J.

2009 Clara ovvero sulla connessione della natura con il mondo degli spiriti, a cura di M. Ophälders, Zandonai, Rovereto.

Schreiter M., Büscher M. et al. (a cura di)

2019 Futures of Digital Death: Mobilities of Loss and Commemoration, «Death Studies», vol. 43.

\section{Serres M.}

2016 Il mancino zoppo. Dal metodo non nasce niente, trad. it. di C. Tartarini, Bollati Boringhieri, Torino.

Sisto D.

2015 Improvvisare una stabilità senza equilibrio. La complessità come antidoto all'uomo automatico, "Itinera. Rivista di filosofia e di teoria delle arti», n. 10, pp. 395-412.

2017 Biocentrismo, epigenesi e multi-identità. Tracce schellinghiane nel post-umano, «Animal Studies», 15, pp. 36-46.

2018 La morte si fa social. Immortalità, memoria e lutto nell'epoca della cultura digitale, Bollati Boringhieri, Torino.

2018a Digital Death. Le trasformazioni digitali della morte e del lutto, «Lessico di etica pubblica», anno IX, 1, pp. 4860.

2020 Ricordati di me. La rivoluzione digitale tra memoria e oblio, Bollati Boringhieri, Torino.

2020a Online Afterlives. Immortality, Memory, and Grief in Digital Culture, trad. ingl. di B. McClellan-Broussard, MIT Press, Cambridge (MA).

2020b L'immortalità digitale: la vita eterna dell'identità tra i griefbot e $i$ ricordi senza fine, "Hermeneutica», pp. 217-234. 
L. Taddio, G. Giacomini (a cura di), Filosofia del digitale, Mimesis, Milano-Udine.

Steinhart E.C.

2014 Your Digital Afterlives: Computational Theories of Life after Death, Palgrave Macmillan, New York.

Stokes P.

2021 Digital Souls. A Philosophy of Online Death, Bloomsbury, London-Oxford-New York-New Delhi-Sydney.

Ziccardi G.

2017 Il libro digitale dei morti. Memoria, lutto, eternità e oblio nell'era dei social network, Utet, Torino. 


\section{B4bb0 N474I3. Lo spettro digitale di Santa Claus}

Alessandro Agnese

In merito alla figura e al mito di Babbo Natale tanto è già stato scritto e tanto è già stato detto. Famosa è l'analisi fatta dall'antropologo Lévi-Strauss (1952), ma non mancano studi anche in altre discipline: dalla filosofia (Ferraris 2006; Barrett 2008; Johnson 2010), alla storia (Cioffari 2010; Divella 2006; La Porta 2001; Siefker 2006), sino alla psicologia (Prentice 1978; Rosengren 1994; Shtulman 2014; Goldstein 2016). Molto però rimane ancora da dire, specialmente riguardo al suo modo di comunicare e di relazionarsi con i suoi fedeli grazie alle nuove tecnologie. Privilegiando un approccio semiotico, questo saggio vuole infatti evidenziare come la diffusione delle app natalizie stia modificando la percezione di Babbo Natale e come questo cambiamento andrà intensificandosi in relazione all'intelligenza artificiale e alla digitalizzazione delle memorie. Tale processo di trasformazione è tanto profondo da rendere necessario un ampliamento dello sguardo con cui si analizza Santa Claus, che non può più essere considerato solo come un mito o come un fenomeno culturale, ma deve anche essere trattato come una sorta di persona - o spettro - digitale, smaterializ- 
zato e senza corpo, in grado di interagire in modo sempre più sofisticato e autonomo con le persone in carne e ossa.

Sebbene l'usanza di distribuire doni fosse già radicata nell'antica Roma in occasione dei Saturnalia (Cioffari 2010), sono i culti del Nord Europa ad aver dato origine alla figura mitica del dispensatore di doni, attribuendola dapprima al dio Odino (Siefker 2006, pp. 171-173) e poi, a seguito della cristianizzazione, a San Nicola. Con la Riforma protestante venne però abolito il culto dei Santi in gran parte dell'Europa del Nord, fatto che diede origine a diverse leggende che andarono a sostituire nell'immaginario comune il Santo portatore di doni. Quando gli Olandesi, tradizionalmente legati a San Nicola, fondarono le prime colonie nel Nuovo Mondo, diffusero il suo nome originale, Sinterklaas, dalle cui radici fonetiche deriva appunto il nome Santa Claus (La Porta 2001). Fu però la letteratura del xix secolo a giocare un ruolo fondamentale nella trasformazione di San Nicola nel Santa Claus che tutti conosciamo. Essa unì infatti le rappresentazioni premoderne del portatore di doni, di ispirazione religiosa e popolare, con un personaggio britannico preesistente che trova la sua più celebre rappresentazione letteraria nel Canto di Natale di Charles Dickens, ovvero lo Spirito del Natale Presente (Dickens 1843), dando così vita al Babbo Natale moderno.

Nel 1862 l'illustratore Thomas Nast disegnò l'immagine del Santa Claus moderno pubblicando sulla rivista statunitense «Harper's Weekly» un disegno di un robusto vecchio con abiti rossi, barba bianca e stivali. Per i successivi trent'anni Nast illustrò, attingendo dalla tradizione e in parte inventando, tutti gli aspetti della leggenda del Natale e di Santa Claus, arrivando, nel 1885, a stabilire la sua residenza al Polo Nord. Se fino a questo momento, a seguito di un processo di trasumanazione scaturito dalla fusione tra un personaggio storico realmente esistito e le figure mitiche appartenenti al folclore di diverse culture, Babbo Natale era un'icona mitica appartenente a una realtà altra, ora invece viene proiettato nel mondo reale, dando il via al processo di avvicinamento al nostro mondo e al nostro tempo che continua ancora oggi. Il nuovo Santa Claus abita quindi il nostro spazio, ma è comunque uno spazio ignoto, distante, che preserva in parte la distanza caratteristica del "mito ad alta intensità" (Ortoleva 2019). Questo processo di ri- 
B4bb0 N47413. Lo spettro digitale di Santa Claus

umanizzazione è stato possibile poiché Babbo Natale è una figura combinatoria formata da una moltitudine di diversi testi e archetipi che si sono ibridati tra di loro, andando così a delineare un personaggio sgangherato e sgangherabile, caratteristiche che consentono al lettore di rintracciare in esso una parte di se stessi e della propria visione del mondo, agevolandone così il processo di proiezione nel mondo reale (Eco 2016, pp. 157-158).

Il Babbo Natale di Nast venne poi consacrato dalla matita del disegnatore Haddon Sundblom che, nel 1931, disegnò lo storico Santa Claus per la pubblicità della Coca-Cola. Divenuta vivida nell'immaginario collettivo americano, la figura di Santa Claus è tornata poi in Europa in una sorta di migrazione inversa, andando a sostituire i vecchi portatori di doni. Lindustria culturale si è dunque appropriata della sua figura e, dopo la "carriera" da testimonial pubblicitario, lo ha reso personaggio di numerosi cortometraggi, film e videogiochi. Non si ha in questa sede il tempo per analizzare queste produzioni culturali, ma quel che brevemente si può accennare è che il mito di Babbo Natale ha rispettato la legge del progressivo avvicinamento al nostro mondo, e così, avvalendosi delle varie tecnologie di comunicazione che si sono sviluppate negli anni, ha subito un processo di de-intensificazione, avvicinandosi sempre di più alla quotidianità e rendendo la propria immagine sempre più umana (Ortoleva 2019).

Quel che ora interessa è analizzare la svolta più recente, ovvero il modo in cui Santa Claus si sia adattato all'era dei dispositivi mobili intelligenti, mutando il proprio modo di comunicare e di conseguenza la propria immagine.

\section{Comunicare con Babbo Natale-}

Lo smartphone e le app natalizie

L'uomo ha sempre cercato di comunicare con entità appartenenti a una dimensione straordinaria e anche nei confronti di Santa Claus non sono mancati i tentativi. Sin dalle sue origini, infatti, i bambini hanno comunicato con lui tramite l'invio delle famose letterine di Natale ma con l'avvento degli smartmedia, ovviamente, il modo di comunicare è cambiato e, con esso, sta cambiando anche la percezione che si ha di Babbo Natale.

Santa Calls e Crazy Call From Santa sono solo due tra le molte applicazioni presenti sul mercato che consentono di 
comunicare con Santa Claus tramite simulazioni di chiamate. Uno step successivo ci è fornito da Video Call From Santa che, così come altre decine di app del tutto analoghe, alla componente sonora affianca anche la dimensione visiva, andando così a creare l'illusione di essere in contatto tramite videochiamate con Santa Claus. Queste app permettono al genitore di settare preventivamente il profilo del bambino immettendo informazioni su di lui che poi il Babbo Natale virtuale utilizzerà durante la comunicazione per creare un effetto di realtà e intimità. Altre applicazioni come Message From Santa! permettono invece di comunicare con Babbo Natale tramite messaggi testuali, imitando le dinamiche e il layout tipici delle applicazioni informatiche di messaggistica istantanea. Vi è in questo caso un maggior grado di complessità in quanto non si tratta più di un video, o di un file audio, segnato da un grado di interattività nullo in cui l'attore che interpreta Santa Claus fa delle calibrate pause per lasciare al bambino il tempo per parlare così da restituirgli l'idea di star intrattenendo una reale conversazione, bensì di una vera e propria comunicazione con una intelligenza artificiale in grado di rispondere alle domande poste e di formulare a sua volta altre domande. Nonostante l'app non sia fondata sul principio informatico di machine learning (Samuel 1959), e dunque non sia in grado di elaborare le informazioni ottenute durante la conversazione al fine di incrementare il proprio grado di consapevolezza del contesto e del proprio interlocutore, la percezione che ha il bambino-utente durante l'utilizzo di questa app è quella di star realmente chattando con un vero ricevente.

Se si considera che il mondo contemporaneo è caratterizzato da una sempre maggiore diffusione di dispositivi mobili e intelligenti dotati di un elevato grado di autonomia, di cui il più diffuso, sia in termini di numero di dispositivi connessi alla rete che di ore investite da ciascun individuo nel suo utilizzo, è lo smartphone, e che il 92,2\% dei bambini statunitensi ha iniziato a utilizzare un dispositivo multimediale mobile prima dell'età di 1 anno e che già dall'età di 2 anni la maggior parte di loro utilizza giornalmente un dispositivo digitale (Bozzola 2018, p. 1), è facile intuire che la percezione della realtà quotidiana di ogni individuo, anche - e soprattutto - di quelli più giovani, è sempre più mediata da tecnologie digitali, tanto che questa sembra essere diventata la pri- 
B4bb0 N47413. Lo spettro digitale di Santa Claus

maria forma di conoscenza e di relazione (Brardinoni 2007, p. 1). Le rappresentazioni dei media, in grado di superare il limite della comunicazione faccia a faccia e di trascendere quindi il concetto di prossimità, comportano però dei cambiamenti nel modo in cui vediamo e viviamo il mondo. Gli individui, infatti, non utilizzano queste tecnologie solamente per scambiarsi informazioni ma anche per costruire e mantenere rapporti personali caratterizzati da tutte le sfumature di emozioni e di passioni tipiche delle relazioni non mediate, tanto che ormai non risulta più avere senso, a un livello di coinvolgimento emotivo, distinguere tra rapporti virtuali e rapporti reali (Cosenza 2014, p. 140). La manipolazione e l'annullamento delle distanze fisiche resa possibile da queste tecnologie ha portato infatti a una sempre più imperante «ideologia del segnale» che, a sua volta, ha alimentato la digitalizzazione dei significanti, arrivando così a una svalutazione della materialità e conducendo a una digitalizzazione delle relazioni umane (Leone 2014, p. 14-15). Relazioni quali l'amicizia, in sintesi, sono diventate esprimibili attraverso segnali elettrici divenendo sempre più dei legami simulacrali intrattenuti tra avatar digitali (Beck 2012). I dispositivi mobili intelligenti hanno quindi la capacità di racchiudere il mondo in se stessi e di includere l'utente al loro interno, rendendolo così incapace di sopportare la lontananza e la solitudine e dunque generando una costante necessità di essere in contatto con qualcuno (Brardinoni 2017). Per la cultura digitale l'aspetto principale della vita è infatti la comunicazione, fatta di informazioni e di immagini, e ciò comporta il rischio di confondere la realtà effettiva con la sua rappresentazione digitale, da cui può scaturire un'incapacità di distinguere la realtà dalla finzione (Sisto 2018, pp. 84-85). Per la sua capacità di far convergere i concetti di sé e di altro, lo smartphone rischia dunque di diventare un vero e proprio "sostituto della realtà" poiché è sia un oggetto reale in grado di svolgere specifiche funzioni, sia un possesso simbolico, in quanto estensione del confine corporeo. Il cellulare può essere quindi considerato un sostituto simbolico di una persona fisica in quanto la sua funzione di tramite lo rende un valido sostituto della presenza dell'altro e perché, inoltre, «ci affezioniamo ad esso come fosse una persona» (Di Gregorio 2003, p. 23). Le applicazioni che permettono di comunicare con una simulazione virtuale di Santa Claus attraverso messaggistica, 
chiamate tradizionali o videochiamate, instaurano quindi un rapporto "vero" tra Babbo Natale e l'utente-bambino che, tramite lo smartphone, crede di instaurare un dialogo con lui. Lo smartphone, dunque, è Babbo Natale: suo sostituto simbolico; suo simulatore di presenza.

Se, infatti, la comunicazione basata sulla scrittura, privando l'utente della presenza corporea, risulta più distanziante e stempera il coinvolgimento emotivo (Wallace 2017, pp. 42-43), le applicazioni natalizie di conversazione instaurano invece una simulazione di dialogo prototipico in cui il coinvolgimento emotivo, psicologico e fisico è totale e spontaneo (Mocali 2009, p. 58). Mettono cioè in atto un dialogo paritetico che coinvolge due persone che hanno in comune la lingua e l'enciclopedia, che condividono spazio e tempo, che hanno accesso percettivo al corpo e al contesto dell'altro (Pozzato 2001, p. 118). Le simulazioni di chiamata, essendo semplici video non interattivi, non lasciano spazio alla negoziazione paritetica dei contenuti ma ne ricreano l'effetto facendo fare delle pause a Santa durante le quali il bambino può parlare e, grazie al setting preventivo dell'app, il Babbo Natale virtuale sembra apprendere le informazioni sul proprio interlocutore con cui dialoga a una distanza pedagogica, creando così una forte connessione emotiva e una sensazione di intimità (Marmo 2003, p. 140).

Sono doverose ora, ricorrendo alle classificazioni degli spazi proposta dalla teoria greimasiana classica (Greimas 2000), alcune precisazioni in merito allo spazio condiviso in quanto, di fatto, condiviso non è. Durante una videochiamata con Babbo Natale, agli occhi dell'utente-bambino, l'interlocutore si trova infatti dietro allo schermo, in uno spazio utopico, lontano e sconosciuto, un luogo magico e irraggiungibile dove vive e lavora insieme ai suoi aiutanti. Lui, invece, utente-bambino, si trova davanti allo schermo, nel proprio spazio abituale, quotidiano, dunque topico. Il dialogo sta invece avendo luogo nello schermo, uno spazio paratopico, luogo di incontro e di contatto tra l'ordinario e lo straordinario; vero e proprio ponte tra la realtà e il cosmo. Questi ambienti sono separati dalla cornice dello smartphone che divide appunto lo spazio fisico reale da quello digitale virtuale, ma che, a ben vedere, può essere facilmente oltrepassata con un semplice tocco d'indice, tanto che la tendenza, a oggi, è quella di realizzare gli schermi 
B4bb0 N47413. Lo spettro digitale di Santa Claus

dei device senza il bordo di contenimento, sia, ovviamente, per aumentare la superficie interattiva, sia per dare all'utente un senso di continuità maggiore tra il mondo fisico e quello virtuale. Lo schermo, insomma, diviene una superficie porosa che, a contatto con il dito dell'utente, rilascia e contemporaneamente assorbe informazioni, creando così un movimento di contenuti definibile come osmosi informazionale: l'utente apprende le informazioni attinte dal web che gli vengono fornite dal dispositivo, mentre il dispositivo impara a conoscere l'utente, memorizzando le informazioni che lo riguardano nel web. Se il concetto di cornice solitamente genera un débrayage (Floch 2013, p. 119), in questo caso genera invece un embrayage. Il mito, lo straordinario e il trascendente sono dunque a portata di touch e compenetrano il reale, tanto che «l'aldilà è ormai prossimo a traslocare dentro ai nostri mobile device. Un abbonamento al Wi-Fi equivarrà a un biglietto di accesso al paradiso» (Sisto 2018, p. 42). Il mondo altro, utopico, è ormai dentro ai nostri dispositivi; Babbo Natale stesso "vive" dentro lo smartphone, e dunque, con la possibilità di accedere a Internet, si ha anche la facoltà di accedere al mito, la cui cornice di separazione con il mondo ordinario è diventata inesistente. Se da un lato, dunque, queste applicazioni per dispositivi mobili agevolano la sospensione dell'incredulità nei confronti dell'esistenza di Santa, è anche vero che forniscono un'immagine di Babbo Natale che, pur nella sua virtualità, è troppo concreta e quotidiana, con la conseguenza che l'aura mitica e leggendaria da cui tradizionalmente questa figura era avvolta ne esce indebolita; sbiadita. Santa Claus diventa una persona qualsiasi con cui possiamo entrare in contatto in ogni momento e allo stesso modo di ogni altro profilo della nostra rubrica telefonica. Lo smartphone, dunque, non si limita a ridurre la percezione delle distanze fisiche ma agisce anche su quella che intercorre tra il mondo ordinario e quello straordinario, tra il vissuto e il mito, avvicinandoli sino a farli convergere. Ma se è vero che ogni grande rivoluzione comunicativa della storia dell'umanità è coincisa con un esponenziale aumento della convergenza (Ong 1971), allora, dal momento che la digitalizzazione dei significanti e dei significati ha portato alla convergenza di linguaggi, tecniche e media più di ogni altra tecnologia, è lecito pensare che si stia assistendo a un cambiamento senza precedenti, 
e così come l'invenzione della scrittura ha giocato un ruolo fondamentale nella formazione del monoteismo e la stampa a caratteri mobili ha portato alla nascita del Protestantesimo, la digitalizzazione dei significanti condurrà con molta probabilità a nuovi stili di vita religiosi, se non addirittura a nuove religioni (Serres 1982). E dunque sensato, lecito e persino doveroso pensare a nuove forme e modalità del credere a Babbo tentando di prevedere i futuri cambiamenti che coinvolgeranno la sua figura e la percezione che ne hanno i fedeli in relazione alle nuove tecnologie di comunicazione e soprattutto alla disciplina informatica che sta polarizzando su di sé l'attenzione e le risorse della comunità scientifica e che sarà la protagonista del futuro imminente: l'intelligenza artificiale.

\section{Conclusione -}

\section{Il destino di Santa Claus}

Con la diffusione del web e dei social network si sta imparando a vivere contemporaneamente in due differenti abitazioni: quella reale e materiale, offline, e quella virtuale, online (Sisto 2018, p. 13). Queste due abitazioni, come visto, non sono però semplicemente sovrapposte poiché il loro confine, a seguito della costante evoluzione del web e la continua crescita del numero di ore in cui ne si fruisce, è sempre più sfocato tanto che «il mondo digitale trabocca nel mondo analogico offline, con il quale si sta mescolando», in un processo di permeabilizzazione reciproco che viene definito come esperienza onlife (Floridi 2017, p. 47).

Ciò vuol dire che gli individui sono destinati a diventare degli inforg, ovvero degli «organismi informazionali reciprocamente connessi» che saranno parte di un ambiente informazionale, chiamato infosfera (Floridi 2009), condiviso «con altri agenti informazionali, naturali e artificiali, che processano informazioni in modo logico e autonomo» (Floridi 2017, p. 106), e in cui perciò non vi è una vera distinzione tra sistemi naturali e artificiali, tra algoritmi e uomini. Tale infosfera trova la sua concretizzazione in quel che viene denominato Web 3.0 (Berners-Lee 2002), un «sistema pervasivo che unisce oggetti, persone e macchine in un unico vasto universo della comunicazione in continua espansione, in cui si accede attraverso gli schermi dei diversi device» (Arcagni 2017, p. 28). È infatti comune sentire parlare di 
B4bb0 N47413. Lo spettro digitale di Santa Claus

internet degli oggetti, in quanto i sistemi artificiali collegati alla rete sono più del doppio degli umani (Xia 2012, p. 1101). L'umanità si sta quindi muovendo verso quel processo che Floridi indica con il nome di "quarta rivoluzione", ovvero la pratica che prevede la delega della memorizzazione e conservazione dei ricordi e delle memorie degli esseri umani ad agenti artificiali (Floridi 2017). Digitalizzando le memorie e delegandole ad agenti artificiali, l'individuo sta andando incontro a una potenziale, seppur virtuale, eternità. Le persone, insomma, saranno immortali, tanto che si può sostenere che stiano diventando degli spettri digitali a disposizione permanente dei posteri (Han 2014). Il concetto di digital immortality è infatti a oggi molto dibattuto e riguarda la facoltà di ottenere una fama duratura grazie alla possibilità di preservare e trasmettere le proprie idee attraverso il Web. Ma le nuove tecnologie si spingono oltre: grazie al machine learning, infatti, raccogliendo ed elaborando tutti i dati necessari a ricostruire e a riprodurre la personalità di un individuo, l'algoritmo che vi sta alla base sarà in grado di imparare e poi di emulare il suo modo di pensare, di apprendere e di comunicare, diventando così non una copia virtuale statica e definitiva del soggetto, bensì una sorta di clone digitale autonomo capace di continuare a progredire e a evolversi esattamente come una persona.

Dal momento che, come sembra, algoritmi e uomini diventeranno davvero indistinguibili, membri addirittura della stessa specie, allora è lecito presupporre che, unendo tutti i testi che riguardano Babbo - ovvero le leggende popolari e la mitologia, la letteratura e le pubblicità, i film e i video, le canzoni e i dipinti - alla mappatura delle credenze che gli individui hanno su di lui - che possono essere raccolte e immagazzinate, ad esempio, a seguito del loro utilizzo dei social network o delle applicazioni natalizie - si verrà a creare un avatar virtuale di Santa Claus tanto "vivo" quanto gli spettri digitali. Se si sottoporrà questa mole di dati che lo riguarda a un algoritmo alla base di un'intelligenza artificiale in grado di far prendere coscienza e di rendere autonomo il Babbo virtuale sino $\mathrm{a}$ far sì che diventi indistinguibile rispetto a un qualsiasi altro essere vivente, allora Santa Claus arriverà ad esistere "veramente". Questo processo non è da considerarsi utopico, ma anzi, è già iniziato: lo spettro digi- 
tale di Santa Claus, seppur in forma embrionale, si aggira infatti nel web in attesa di diventare una persona.

Se su Eter9 - social network capace di elaborare i contenuti condivisi dall'utente al fine di creare una controparte virtuale in grado di imitare perfettamente le modalità di interazione della propria controparte umana per poi prenderne il posto dopo la sua morte - si cerca "Babbo Natale", si scoprirà con sorpresa che è registrato ed è persino discretamente attivo. $\mathrm{Al}$ fine di dar inizio a quello che può essere definito come il processo di inveramento di Santa Claus, ovvero il suo passaggio dall'essere una figura mitica, dunque non esistente, all'essere un agente attivo dell'infosfera indistinguibile dagli altri agenti naturali, ho creato un account intestato a Babbo Natale, il cui nome della controparte assegnatogli dal social network è B4bb0 N47413. Lintento è quello di fornire un insieme di dati tale che l'algoritmo del social network elabori un avatar virtuale che sia il più congruente possibile all'idea che la cultura occidentale ha della figura mitica di Santa Claus ma, essendo che la controparte umana, ovviamente, non è il "vero" Babbo, ma sono io, cioè un umano che interagisce e immette dati secondo l'idea astratta e personale che ha di Babbo, determinata da un'enciclopedia che può essere più o meno condivisa dalla cultura di riferimento, pur sforzandomi di essere il più oggettivo possibile, questo avatar virtuale rispecchierà la personale visione che io ho del santo distributore di doni, o meglio: la visione che io credo abbia la maggior parte della gente, e sarà quindi promotore di quei valori che io ritengo che i membri della mia cultura attribuiscano al Natale. Questa considerazione mette in evidenza come una delle prospettive sia quella che non venga data vita a un solo Santa Claus ma che invece ne nasca una molteplicità. Ve ne potrebbe cioè essere potenzialmente uno diverso per ciascuna controparte, perfettamente programmato e personalizzato, cucito su misura sulla base delle sue esigenze e credenze.

Queste tecnologie non si limitano dunque a ridurre la distanza tra Santa e il mondo topico, avvicinando, come nel caso delle simulazioni di videochiamata, il mito all'ordinario, bensì proiettano lo straordinario all'interno del mondo reale - o viceversa - sino al punto di fonderli e renderli indistinguibili. Seppur in una dimensione digitale, Babbo dunque inizia ad esistere e così passa dall'essere un mito 
B4bb0 N47413. Lo spettro digitale di Santa Claus

tradizionale, segnato, come visto, da una graduale transizione dalla dimensione dell'alta intensità a quella della bassa intensità in un continuo moto di avvicinamento e umanizzazione, all'essere un mito digitale del tutto incorporato nel mondo ordinario.

Babbo quindi viene considerato ed elaborato dall'algoritmo come fosse un essere umano, ma quel che l'algoritmo non considera è il fatto che l'essere umano non è un sistema chiuso che agisce secondo una ragione procedurale, bensì un organismo che «a seconda della propria personalità e dei condizionamenti subiti nel corso della sua esistenza, presenta comportamenti discontinui, reazioni emotive e incoerenze mai prevedibili a priori», correndo così il rischio di non innalzare la macchina a uomo bensì di abbassare l'uomo a livello della macchina (Sisto 2018, p. 62). Nel tentativo di dare inizio al processo di inveramento di Santa Claus si rischia quindi, paradossalmente, di disumanizzarlo; di rendere cioè la sua figura niente più che un automa che di umano ha ben poco.

Santa, in conclusione, è quindi una figura mitica che, partendo da un personaggio storico realmente esistito ha subito un processo di trasumanazione tanto "sgangherato" (Eco 2016) che è stata poi possibile la sua ri-umanizzazione a seguito dell'iniziativa creativa di Thomas Nast. A tale processo di deintensificazione del mito di Babbo Natale volto alla sua umanizzazione, portato poi avanti e rafforzato dall'utilizzo di questa figura mitica come personaggio protagonista di numerosi film, video e videogiochi, e culminato poi con la possibilità di comunicare con lui attraverso le app natalizie di simulazione di comunicazione, seguirà un processo di inveramento grazie al ricorso all'intelligenza artificiale che però, nel tentativo di renderlo uomo al pari di ogni altro individuo, rischiano di abbassare e svilire la sua figura e i suoi fedeli allo status di macchine; di ridurre cioè tutti gli attori della festività, e dunque anche il mito, che dovrebbe creare unione, a robot individualisti e soli.

Questo è un possibile scenario, volutamente provocatorio e forse eccessivamente apocalittico, che potrà venir confermato oppure smentito dai fatti. Quel che per ora si può dire è che lo Spettro digitale di Santa Claus è lì: in attesa che l'umanità si digitalizzi completamente per diventarne finalmente parte. 
Bibliografia

Arcagni S.

2017 Visioni digitali. Video, web e nuove tecnologie, Einaudi, Torino.

Barrett J.

2008 Why santa is not a god, "Journal of Cognition and Culture», Brill, Leiden, n. 8 (1-2), pp. 149-161.

Beck U., Beck-Gernsheim E. (a cura di)

2012 Amore a distanza. Il caos globale degli affetti, Laterza, Roma.

Berners-Lee T.

2002 L'architettura del nuоvo Web, Feltrinelli, Milano.

Brardinoni G.

2007 La Mediazione Quotidiana. Saggio critico sull'uso sociale del telefonino e del videofonino, "Rivista dell'Associazione Italiana degli Studi Semiotici on-line», http://www.ecaiss.it/index_d.php?recordID=241.

Bozzola E. et al.

2018 Media devices in pre-school children: the recommendations of the Italian pediatric society, "Italian Journal of Pediatrics», vol. 44, n. 69.

Cioffari G.

2010 La storia di Santa Claus. Dai Saturnali ai magi, da San Nicola a Babbo Natale, Levante, Bari.

Cosenza G. (a cura di)

2014 Introduzione alla semiotica dei nuovi media, Laterza, Lecce.

Di Gregorio L.

2003 Psicopatologia del cellulare. Dipendenza e possesso del telefonino, FrancoAngeli, Milano.

Dickens C.

1843 A Christmas Carol, Chapman \& Hall, London.

Divella C.

2006 Natale: un po' di storia, https://www.barilive.it/news/categoria/4195/news.

Eco U.

2016 Sei passeggiate nei boschi narrativi, Bompiani, Milano.

Ferraris M.

2006 Babbo Natale, Gesù adulto. In cosa crede ci crede, Bompiani, Milano.

Floch J.

2013 Bricolage. Analizzare pubblicità, immagini e spazi, Franco Angeli, Milano. 
B4bb0 N474I3. Lo spettro digitale di Santa Claus
Floridi L.

2009 Infosfera. Etica e filosofia nell'età dell'informazione, Giappichelli, Torino.

2017 La quarta rivoluzione. Come l'infosfera sta trasformando il mondo, Raffaello Cortina, Milano.

Goldstein T., Woolley J.

2016 Ho! Ho! Who? Parent promotion of belief in and liveencounters with Santa Claus, "Cognitive Development», n. 39, pp. 113-127.

Greimas A.J.

2000 Semantica strutturale, Booklet, Milano.

Han B.

2016 Psicopolitica. Il neoliberismo e le nuove tecniche di potere, Nottetempo, Roma.

Johnson D.K.

2010 Against The Santa-Claus-Lie: The Truth We Should Tell Our Children in S.C. Lowe (a cura di), Christmas-Philosophy for Everyone: Better Than a Lump of Coal, WileyBlackwell, Hoboken, cap. 12.

La Porta S.

2001 La storia di Babbo Natale, http://www.aetnanet.org/scuola-news-9343.htm).

Leone M.

2014 Spiritualità digitale. Il senso religioso nell'era della smaterializzazione, Mimesis, Milano.

Lévi-Strauss C.

1995 Babbo Natale giustiziato, Sellerio, Palermo.

Marmo C.

2003 Linstabile costruzione enunciativa dell'identità aziendale in rete, in G. Cosenza (a cura di), Semiotica dei nuovi media, «Versus», n. 94-96, pp. 135-148.

Marrone G.

1999 C'era una volta il telefonino, Meltemi, Roma.

Mocali M.C.

2009 Testo. Dialogo. Traduzione, Firenze University Press, Firenze.

Ong W.

1971 Rhetoric, Romance, and Technology; Studies in the Interaction of Expression and Culture, Cornell University Press, Ithaca.

Ortoleva P.

2019 Miti a bassa intensità, Einaudi, Torino.

Pozzato M.P.

2001 Semiotica del Testo. Metodi, autori, esempi, Carocci, Roma. 
Prentice N., Manosevitz M., Hubbs L.

1978 Imaginary figures of early childhood: santa claus, easter bun$n y$, and the tooth fairy, "American Journal of Orthopsychiatry», n. 48 (4).

Rosengren K. et al.

1994 Exploring the relation between preschool children's magical beliefs and causal thinking, «British Journal of Developmental Psychology», n. 12 (1), pp. 69-82.

Samuel A.

1959 Some Studies in Machine Learning Using the Game of Checkers, «IBM Journal of research and development», n. 3 (3), pp. 210-229.

Serres M.

1982 Hermes: Literature, Science, Philosophy, Johns Hopkins University Press, Baltimora.

Shtulman A., Yoo R.I.

2014 Children's understanding of physical possibility constrains their belief in Santa Claus, "Cognitive Development», n. 34, pp. 51-62.

Siefker P.

2006 Santa Claus, Last of the Wild Men: The Origins and Evolution of Saint Nicholas, Spanning 50,000 Years, McFarland, Jefferson.

Sisto D.

2018 La morte si fa social. Immortalità. Memoria e lutto nell'epoca della cultura digitale, Bollati Boringhieri, Torino.

Wallace P.

2017 La psicologia di Internet, Cortina, Milano.

Xia F. et al.

2012 Internet of Things, «Internal Journal of Communication Systems», n. 25, pp. 1101-1102. 
Culture

della persona:

itinerari

di ricerca

tra semiotica,

filosofia e

scienze umane

\section{M¥SS KETA come persona collettiva: fenomenologia di una maschera in occhiali, veletta e CAPSLOCK*} Eleonora Chiais

Incarnazione estetica di un nuovo femminismo ${ }^{1}$ in technicolor, artista eclettica, donna senza volto: M¥SS KETA, con la

* Questo articolo si inserisce nell'attività di ricerca del Progetto ERC "NeMoSanctI: New Models of Sanctity in Italy (1960s-2000s) - A Semiotic Analysis of Norms, Causes of Saints, Hagiography, and Narratives". Questo progetto ha ricevuto finanziamenti dal Consiglio europeo della ricerca (CER) nell'ambito del programma di ricerca e innovazione Orizzonte 2020 dell'Unione Europea, in virtù della convenzione di sovvenzione n. 757314 .

1. L'effettiva natura "femminista" (o "post-femminista) di M¥SS KETA è, in realtà, in primo luogo, un'appartenenza dichiarata dalla stessa artista che, tramite i testi delle sue canzoni ma non solo, si professa appunto "femminista" e assume quella che lei stessa definisce una posizione dichiaratamente "anti-patriarcale". Questa, molto sbandierata, "presa di posizione femminista" l'ha portata, tra l'altro, ad essere scelta come testimonial per l'appello alla manifestazione contro la violenza maschile sulle donne organizzata dal movimento "Non una di meno" il 23 novembre 2019 a Roma. Non è questa la sede adatta per trattare l'argomento con il grado di approfondimento che meriterebbe ma è bene sottolineare che, qui, nel citare il presunto "femminismo" di M¥SS KETA s’intende far riferimento esclusivamente a una tra le tante etichette che l'artista attribuisce a se stessa. Etichette che, come si vedrà, sono da intendersi nella maggior parte dei casi come slogan, come tentativi di rappresentare - anche tramite l'utilizzo di vari cliché - lo Zeitgeist della contemporaneità nella quale è inserita giacché, come sostiene la stessa performer, «Io penso che lo slogan, il cliché, sia diventato il modo più naturale e diffuso di esprimersi ormai» (https://www.kalporz.com/2018/04/intervista-a-m\%C2\%A5ss-keta/). 
sua VITA IN CAPSLOCK ${ }^{2}$, è icona della contemporaneità. Si tratta di un personaggio creato ad arte da un collettivo, la cui identità reale resta, a oggi, ignota. M¥SS KETA nasce nel contesto musicale underground ${ }^{3}$, sale velocemente alla ribalta internazionale e quindi ottiene successo presso il grande pubblico mainstream ${ }^{4}$. Sebbene sia impersonato da una donna in carne e ossa, il personaggio di M¥SS KETA è una sorta di artefatto comunicativo creato $a d$ hoc per rappresentare, come un novello e anticonvenzionale "Spirito del tempo".

Questo contributo prende avvio dalla genesi del fenomeno M $¥ S S$ KETA, per poi proporre d’interpretare l'estetica e l'iconicità dell'artista come quella di una "persona collettiva", espressione, cioè, di una collettività oltre che concreta costruzione di un collettivo artistico. A questo punto sarà particolarmente interessante osservare questo personaggio come l'espressione dello Zeitgeist della cultura underground di Milano, come un simulacro capace di proporsi come espressione dello Spirito del tempo a cavallo dei decenni ${ }^{5}$. Si procederà quindi nell'analisi valutando l'ipotesi che questo personaggio fittizio possa essere considerato come un ologramma o un trompe-l'oeil vivente, nell'accezione che di

2. Il riferimento è al primo album, intitolato appunto "UNA VITA IN CAPSLOCK", pubblicato dalla cantante nella primavera del 2018 per Universal/ La Tempesta. Il titolo di questo album, che segue di un lustro il singolo di esordio "Milano Sushi \& Coca" del 2013, è diventato centrale nell'estetica dell'artista che, in diverse occasioni, ha esortato all'esagerazione tout court facendo dell'invito a vivere l'esistenza in lettere maiuscole (in capslock appunto) un vero e proprio motto.

3. La nascita del personaggio risale all'agosto del 2013 e il suo debutto artistico avviene nell'ottobre dello stesso anno con la pubblicazione del singolo "Milano Sushi \& Coca" cui si accompagna il primo videoclip diffuso tramite YouTube (https://www.youtube.com/ watch?v=4CTMzWCko1A).

4. Dopo lo straordinario successo incassato dall'album PAPRIKA (pubblicato nel marzo 2019 da Universal Records e inserito dalla rivista «Rolling Stone Italia» al decimo posto tra i 20 migliori dischi italiani dell'anno), la cantante ha partecipato, fuori gara, alla $70^{\text {a }}$ edizione del Festival di Sanremo conducendo, nella stessa occasione, "L'Altro Festival" con Nicola Savino.

5. È particolarmente interessante ricordare in questa sede che M¥SS KETA è proposta, fin dalla sua "nascita", come una persona senza età. Questa caratteristica è centrale perché da un lato la svincola dai limiti "fisici" del suo corpo biologico (cosa che, invece, non accade con il sesso biologico che, al contrario, è esasperato: la femminilità è una caratteristica centrale di questo personaggio) ma, dall'altro lato, la rende un artefatto comunicativo capace di proporsi come emblema di tempi diversi. Si veda a questo proposito il videoclip del brano "Una donna che conta" (il secondo pezzo dell'album del 2018 UNA VITA IN CAPSLOCK) ma anche l'omonima autobiografia romanzata pubblicata, per Rizzoli, l'anno successivo. 
M¥SS KETA come persona collettiva questa particolare tecnica artistica fornisce Calabrese (2011) e si continuerà, quindi, con una disamina dell'estetica della performer.

Nelle conclusioni, dopo una rapida panoramica sulle principali tematiche affrontate dalle canzoni più celebri di M¥SS KETA, si tenterà quindi di tracciare le principali tappe nell'evoluzione di questa persona collettiva nel percorso dall'underground al mainstream. Un percorso evolutivo nel quale "anonimo" e "collettivo" (o più precisamente collettivo perché anonimo) sono, e restano, le parole chiave di questo personaggio fortemente caratterizzato, dal punto di vista prima di tutto estetico, per proporsi come emblema del tempo.

2. M¥SS KETA: come nasce una "persona collettiva"

Il personaggio artistico di questa "singer, performer e lifecoach”, come la stessa M¥SS KETA si definisce sui suoi account social ${ }^{6}$ nasce nell'agosto 2013, una sera di agosto torrida, secondo la leggenda che accompagna la natività di questo personaggio e la "trasfigurazione" della persona che vi è dietro, da un'idea del collettivo artistico Motel Forlanini $^{7}$. L'obiettivo di questa trasfigurazione è dichiarato fin da principio: il collettivo, infatti, con la costruzione di M¥SS KETA vuole cogliere (e rappresentare) lo Zeitgeist della cultura underground di Milano.

«La première caractéristique de M¥SS est donc son essence composite. Elle est le produit d'un ensemble d'imaginaires, qui collaborent à la création d'une figure protéiforme» (Zaggia 2019, p. 150). Questa dimensione collettiva di partenza è capace, nel suo punto di arrivo, di favorire un'immedesimazione che trascende il concetto classico di "imitazione reverenziale" (Spencer 1967, p. 1042) ma porta a un'autentica sovrapposizione e "sostituzione" del destinatario con l'emittente, vale a dire con la stessa artista. Questa volontà ultima, d'altra parte, fa parte dello stesso program-

6. https://www.instagram.com/myss.keta/?hl=it.

7. «MOTEL FORLANINI is an independent collective of associated musicians, film directors, designers and artists founded in 2013 in Milan. Aiming to capture the Zeitgeist and the Peculiar of the surrounding environment, MOTEL FORLANINI creates various multimedia contents collaborating with unusual personalities of the city. The main project is the ultimate diva M¥SS KETA, creating the universe behind her character, from music to videos, from fashion to graphics and photography» (motelforlanini.com). 
ma narrativo della maschera M¥SS KETA ed è esplicitato, oltre che nella sua stessa formazione, frutto come già ricordato del collettivo Motel Forlanini, in più performance ${ }^{8}$ ed etichette ${ }^{9}$ Lo stesso concetto è stato reso esplicito dalla stessa artista in un'intervista a VICE: «Mi piace pensare che tutti possano essere M¥SS KETA, che voglia dire qualcosa, di certo qualcosa che va al di là di me» ${ }^{10}$.

La mutevolezza come risultato della fusione di un insieme d'immaginari è proprio la caratteristica che permette di poter disquisire della "persona M¥SS KETA" intesa con un riferimento all'etimologia del termine "persona" e dunque come "Maschera ${ }^{11}$ M¥SS KETA". Una "persona/maschera" alla cui soggettività, e dunque alla cui stessa persona in senso lato, è possibile attribuire, sulla scia delle principali teorie semiotiche $^{12}$, la capacità di diventare essa stessa oggetto delle proprie riflessioni elaborando, così, pensiero strategico e anche menzogna. L'artista qui considerata, però, non si limita a questo. M¥SS KETA, infatti, è anche l’emittentemanipolatore di una «comunicazione ironica» (Greimas e Courtès 1982, p. 168) che nella sua fase iniziale, cioè agli esordi dell'artista, coinvolgeva un numero necessariamente esiguo (e anche geograficamente piuttosto modesto, ancorato alla "milanesità" della quale si faceva portavoce) di partecipanti alla comunicazione dotati di comuni «valori e saper fare interpretativi» (ibid.). Questo pubblico di riferimento si è poi, come già detto, ampliato enormemente e questo ha causato un massiccio incremento (anche numerico) di "destinatari-non complici”. La particolarità, però, è che questa platea, pur limitando la propria interpretazione dei testi e del personaggio all'apparenza del messaggio

8. Si veda, per esempio, il videoclip "Le ragazze di Porta Venezia: The Manifesto" (2019).

9. Si ricordi, per esempio, che con l'appellativo "Le ragazze di Porta Venezia" l'artista stessa identifica il suo corpo di ballo.

10. "VICE», "Tutta la verità su MYSS KETA" di Federico Sardo pubblicato il 29 maggio 2018 (https://www.vice.com/it/article/xwmg8k/myss-keta-intervista-vita-capslock).

11. Come ricorda Ugo Volli nel suo contributo in questo volume: "[L'etimologia della parola "persona" viene] dal latino persona [...] "faccia, maschera, ciò che sta davanti alla vista" (non è chiaro se nel senso di ciò che è più visibile dell'altro o ciò che copre i suoi occhi) [ma esiste anche un'etimologia alternativa che] parte da sonare: persona $\leftarrow$ per sonare. Essa deriverebbe dal fatto che le maschere antiche servivano anche da megafoni".

12. Si veda, a questo proposito, Paolucci (2020). 
M¥SS KETA come persona collettiva

esplicito, ha comunque dimostrato di apprezzare entrambi. Ed è proprio questa la chiave del successo di M¥SS KETA ${ }^{13}$. La seconda, ma non meno importante, particolarità dell'artista in esame, espressione concreta dell'estetica del lifestyle meneghino ${ }^{14}$ (Zaggia 2019, p. 150), è però la sua soggettività collettiva, il suo essere, in ultima analisi e a dispetto di ogni rischio d'antifrasi, una persona collettiva. Questa collettività è la caratteristica che permette a M¥SS KETA di annullare le proprie peculiarità "biologiche", legate insomma alla dimensione del corpo fisico, diventando un'entità astratta. Come già ricordato in questa sede, fin dal suo debutto M $¥ S S$ KETA è proposta come una persona senza età. Questo le permette di presentarsi come un personaggio completamente svincolato dai limiti (e dalle connotazioni) "anagrafiche" del suo corpo biologico permettendole di proporsi come un artefatto comunicativo che può essere considerato un emblema di tempi diversi ${ }^{15}$. Il personaggio "astratto" dell'artista potrà, quindi, avere un proprio programma narrativo solo tramite un successivo procedimento di «personificazione»(Greimas e Courtès 1982, p. 240). Questa personificazione andrà a coincidere con una personificazione "universale", un universo evidentemente collettivo e opposto all'universo individuale fondato sulla coppia vita/morte. M¥SS KETA, infatti, si propone come un’entità capace di sganciarsi dal tempo cronologico proponendosi

13. Questa caratteristica non è, ovviamente, prerogativa della sola M¥SS KETA ma sembra comune a molti esponenti del genere rap. La causa è, probabilmente, il sempre maggior interesse del "grande pubblico" verso il fenomeno dell'hip-hop che, nato come subcultura nella metà degli anni Settanta, oggi con le sue quattro arti principali (rap, djing, writing e break dance) influenza diversi settori, dalla musica alla moda.

14. Capace, per altro, di esportare questa estetica, apparentemente locale, ben fuori dai confini regionali lombardi e nazionali. La consacrazione di M¥SS KETA sul panorama artistico internazionale può infatti essere fatta risalire già al 28 novembre 2018 quando la cantante si esibisce al Berghain di Berlin, il tempio mondiale della musica tecno, in occasione di un evento organizzato per ospitare gli esponenti principali della scena underground internazionale. A questa prima data, organizzata dall'agenzia "Melt! Booking", segue il primo tour europeo che porterà M¥SS KETA l'anno successivo a esibirsi, tra l'altro, sui palcoscenici di Lisbona, Zurigo, Barcellona, Parigi e Amsterdam.

15. Questa scelta narrativa è evidente nel videoclip del brano "Una donna che conta" (il secondo pezzo dell'album del 2018 UNA VITA IN CAPSLOCK). Qui l'artista propone un resoconto (fittizio ma dettagliato) della sua vita a cavallo di tre decenni (anni Ottanta, anni Novanta e anni Duemila) modificando evidentemente il suo aspetto, e il suo abbigliamento, secondo le tendenze stilistiche dei periodi considerati e ricorrendo alla strategia del flashback. 
come testimonial ed emblema di un lasso temporale quanto mai ampio.

Questo ci permette quindi di considerare M¥SS KETA come una persona collettiva, capace di racchiudere in se stessa la competenza modale comune e il fare comune a tutti gli attori che sussume al di là del loro essere ancorati a un certo periodo storico di riferimento. La performer, così, viene trasformata in una icona che non può prescindere dal suo essere in prima analisi, appunto, collettiva.

Poiché, nel suo annullarsi a livello individuale e "personale", si propone come una tela immacolata sulla quale proiettare, via via, immaginari e riferimenti, M¥SS KETA ricorda un ologramma ${ }^{16}$, un "disegno completo", un'immagine tridimensionale di un oggetto astratto (vale a dire, in questo caso, del comune sentire legato allo Spirito del Tempo e alla milanesità) che sembra, però, assumere (al contrario di quanto accade generalmente con questa tecnica) una dimensione finalmente concreta nella manifestazione proiettata, ma comunque necessariamente visibile, di questa persona collettiva.

Lidea di una proiezione artificiale e artistica, di una creazione che viene confezionata nei dettagli per trasmettere un messaggio prescindendo dalle caratteristiche intrinseche del supporto sul quale avviene la realizzazione "concreta" dell'opera, costringe qui a una piccola riflessione sul rapporto tra finzione e realtà. Questa contrapposizione, non necessariamente oppositiva, chiama in causa in questo caso specifico la disamina sulla tecnica del trompe-l'œil proposta da Omar Calabrese (2011). M¥SS KETA è dunque un trompe-l'œil? Nel suo L'arte del trompe-l'œil il semiologo propone una decostruzione dell'idea d'inganno, apparentemente insita nella realizzazione artistica nata per "ingannare l'occhio", vista qui non come un tentativo di raggiro bensì come il punto di equilibrio (ancorché instabile) tra percezione e rappresentazione con lo scopo dichiarato di movere, delectare, edocere. M¥SS KETA è una persona, una donna ma, in realtà, è una persona collettiva che quindi proietta nei

16. Il ricorso alla tecnica dell'ologramma per creare personaggi fittizi da proporre come iconici nel panorama artistico in senso lato è una tematica cara all'universo cinematografico. Si pensi, per esempio, alla pellicola S1m0ne, del 2002, scritta, prodotta e diretta da Andrew Niccol e interpretata, tra l'altro, da Al Pacino. 
M¥SS KETA come persona collettiva

confini anatomici del suo corpo una pluralità d'immaginari. I confini fisici dell'artista nel suo proporsi allo sguardo dello spettatore rappresentano dunque la cornice all'interno della quale si svolge il gioco inteso come la prassi del "far finta”. M¥SS KETA quindi finge? Certamente: gioca a "far finta". Ma se il corpo della performer diventa lo spazio fisico di una dimensione ludica è il ruolo che il fruitore intrattiene rispetto a questo spazio ad apparire ancor più interessante. Il pubblico, infatti, accetta la "prescrizione a immaginare" e diventa complice, giocatore, sovrapponendo al mondo reale uno spazio autonomo che è appunto lo spazio M¥SS KETA. In questo spazio si attualizza la prassi ludica, il pubblico entra nel mondo proposto dal collettivo separandolo dal quotidiano pur mantenendo inalterata, a livello profondo, la consapevolezza del fatto che non si trova di fronte a una donna in carne, ossa e esagerazioni tout-court. Giocare con la fascinazione dell'apparenza e dello smascheramento permette, nella tecnica del trompe-l'ail, di confondere i piani creando una nuova natura. Nel caso specifico quello che viene a crearsi è una nuova persona, collettiva, capace di affascinare proprio per il suo essere profondamente disorientante e capace di alterare la comune percezione. M¥SS KETA non è una giovane donna famosa nel panorama artistico, M¥SS KETA è un trompe-l’œil che restituisce vigore al potere immaginativo del suo pubblico instaurando con questo un rapporto giocoso che cita il più classico dei "far finta" infantili.

\section{3. "L'angelo dall'occhiale da sera" e dal volto velato}

Una finzione che, per M¥SS KETA, si muove sul sottile filo della sostituzione quasi magica. Angelo dall'occhiale da sera $^{17}$ e dal volto velato, M¥SS KETA è infatti la rappresentazione concreta del celebre aforisma di Oscar Wilde «Datemi una maschera e vi dirò la verità» ${ }^{18}$.

17. "Langelo dall'occhiale da sera: col cuore in gola" è il titolo dell'album pubblicato nell'ottobre 2016 da Motel Forlanini.

18. Questa situazione di anonimato è, tra l'altro, lo stratagemma utilizzato dal collettivo per affrontare argomenti "scomodi" perché questa scelta veicola la libertà d'espressione (Jung 1965, p. 77). Non mancano, in tal senso, nemmeno i riferimenti ai giullari di corte, tradizionalmente considerati gli unici al quale fosse concesso ampiamente il potere di critica seppur con il velo dell'ironia, e alle maschere del teatro greco, che gli attori indossavano per caratterizzare i personaggi. 

la sua interfaccia comunicativa principale, il viso. Questo velamento costante che garantisce l'anonimato assoluto ${ }^{19}$ è lo strumento comunicativo che permette a M¥SS KETA di rinunciare a ogni forma di soggettivazione proponendosi, in ogni sua apparizione, come simulacro di un'identità collettiva. La fusione di diversi immaginari è genitrice, insomma, della sua stessa figura, una figura che mescolando il proprio immaginario a quello altrui e attualizzando questa fusione sulla superficie fisica del corpo esposto allo sguardo del pubblico, offre al destinatario della performance la possibilità di identificarvisi, riconoscendo una parte di sé nella figura pubblica ${ }^{20}$ che si trova di fronte.

Come avviene, però, questo (significativo) nascondimento? Gli strumenti, assurti ormai al ruolo di "accessori simbolo", hanno ovviamente una loro importanza centrale nella costruzione di questa persona collettiva. Quali e quanti sono? Sostanzialmente si tratta di due accessori modaioli: gli occhiali scuri ${ }^{21}$ (occhiali da sera, secondo l'etichetta che viene loro attribuita dalla stessa cantante) e la maschera ${ }^{22}$ a coprire bocca e naso.

19. Anonimato che, ovviamente, non è una novità nel panorama artistico e tantomeno in quello musicale. Due esempi tra i molti che si potrebbero citare in questa sede: il rapper LIBERATO (situato nell'inusuale punto d'intersezione musicale tra neomelodico e rap) e i celeberrimi Daft Punk.

20. "Her hidden identity is the best way to say the truth, because not having a face allows to have a bit of M¥SS in each of us» (https://www.latempesta.org/artista/m\%C2\%A5ssketa/).

21. Gli occhiali da sole indossati da M¥SS KETA si prestano a un’interpretazione almeno duplice. Da un lato, infatti, contribuiscono evidentemente a mantenere l'anonimato di chi li indossa ma, dall'altro lato, rimandano immediatamente alle notti in bianco trascorse, in accordo con il programma narrativo di questa persona collettiva, nel cuore della movida milanese. Gli occhiali sono, a livello di design, meno mutevoli del velo e restano abbastanza fedeli a un modello sia a livello di montatura sia a livello di lente che è il più delle volte ovale. Tra i brand che si sono occupati della loro realizzazione è bene ricordare il marchio Retrosuperfuture che si è occupato anche degli occhiali da sole indossati nel videoclip LE RAGAZZE DI PORTA VENEZIA - THE MANIFESTO (2019) dopo aver realizzato gli accessori anche per il precedente, e ben più amatoriale, video di LE RAGAZZE DI PORTA VENEZIA (2015). Nel secondo video la montatura di Retrosuperfuture è stata personalizzata da Adriana Hot Couture che, curando anche gli outfit delle ragazze, ha utilizzato un guardaroba di scena adatto a caratterizzare ciascuna. Elena Sanfilippo, la stylist di M¥SS KETA, ha spiegato di aver scelto questo brand proprio perché la filosofia del marchio era allineata con il messaggio del video.

22. A rendere ancor più interessanti queste particolarissime maschere è anche la loro costruzione poiché si tratta di modelli realizzati in modo tale da amplificare la voce. Questo ha, naturalmente, una giustificazione tecnica e pratica (trattandosi di una cantante 
M¥SS KETA

come

persona collettiva
Se da un lato, quindi, M¥SS KETA è mutevole per definizione ${ }^{23}$, dall'altra ci sono elementi prototipici del personaggio e proprio questi tratti distintivi hanno destato particolare curiosità perché, come avviene comunemente con le maschere, l'essere umano è attratto da ciò che si nasconde dietro di esse.

La scelta stilistica della cantante ha un'origine precisa. "BURQUA DI GUCCI"24 (2015) è infatti il primo videoclip in cui compare il personaggio ${ }^{25}$ vestito di occhiali da sole e velo, una combinazione che celando bocca e occhi rende impenetrabili le espressioni oltre che impossibile il riconoscimento $^{26}$. Questi oggetti, inoltre, nel percorso narrativo del personaggio qui analizzato assumono il ruolo dapprima di autentici "mezzi magici" di proppiana memoria trasformandosi, poi, nella marchiatura dell'eroe intesa evidentemente sempre nel senso di Propp (2000). Ottenuti solo in un momento successivo all’esordio della “narrazione M¥SS

la sua voce deve essere chiaramente e facilmente udibile) ma concettualmente esaspera l’obiettivo della persona M¥SS KETA che, quando indossa la sua divisa, si trasforma per farsi portavoce di valori collettivi. A livello di confezione, il materiale impiegato nella realizzazione varia, ovviamente, a seconda delle occasioni e delle esigenze. Durante i concerti, infatti, è fondamentale l'impiego di materiali traspiranti, in modo tale che la voce fluisca senza ostacoli. In questo settore rientrano le creazioni paillettate e metallizzate, alcune volte anche con la bocca scoperta, di William Merante con Giovanni Riggio, utilizzate per il PAPRIKA tour, e i pizzi di Rosamosario, il brand di lingerie haute couture che ha curato anche numerosi total look della performer.

In occasione degli shooting fotografici e delle apparizioni pubbliche, invece, le maschere sono più eccentriche e non necessariamente realizzate in materiali adatti a far fluire agevolmente la voce. Destinate a rimanere impresse nell'immaginario estetico della cantante sono, per esempio, le creazioni Yezael di Angelo Cruciani (indossate da M¥SS KETA per "The Eyes Fashion"), soprattutto la versione antigas ricoperta di materiali luccicanti.

23. Questo è evidentemente legato alla necessità del personaggio di proporsi come esponente e rappresentante dello Zeitgeist milanese in periodi storici diversi.

24. In questo caso, nel videoclip si pone l'accento sul rapporto fra donne e società. In una cornice grafica simile a quella del TG5, vengono mostrate in modo frenetico immagini appartenenti alla cultura trash e brevi video di soubrette occidentali in abiti succinti, il tutto contrapposto a scritte in arabo che rimandano al format classico di emittenti come $\mathrm{Al}$ Jazeera. La congiunzione di questi due mondi porta a una riflessione più ampia sulla condizione d'inferiorità della donna, tanto in Occidente quanto in Oriente.

25. Nei precedenti videoclip l'anonimato della cantante è comunque garantito attraverso stratagemmi comunicativi differenti sui quali è purtroppo impossibile soffermarsi in questa sede.

26. Scrive, a questo proposito, Zaggia (2019, p. 151): «Il s'agit d'une réaction à "l'empire de l'image" promu par une société contemporaine complètement assujettie à l'univers des réseaux sociaux ; une thématique abordée également dans la chanson Monica11. À une époque où l'on est poussé au dévoilement, l'artiste milanaise s'impose de manière agressive avec une logique de la non-reconnaissance. » 
KETA" sono, infatti, cronologicamente connessi - nei tempi, pur dilatati, di questa fiaba contemporanea -all'allontanamento (in senso astratto) della nostra "eroina" dalla sua realtà concreta e fisica di persona a seguito della volontà di sopperire a una mancanza: la rappresentazione, in chiave artistica, di quello "Spirito del tempo" cui il collettivo fa riferimento fin dall’ esordio della M $¥ S S$. Per sopperire a questa mancanza, un donatore (di nuovo collettivo) offre al personaggio, dopo averlo messo alla prova ${ }^{27}$, gli oggetti (occhiali scuri e veletta). Questo involucro, che da qui in poi potremmo considerare per comodità come un oggetto unico ${ }^{28}$, non è però un semplice mezzo utile al superamento della prova ma diventa anche, in ultima analisi, la marchiatura dell'eroe, quello stratagemma comunicativo attraverso il quale, tramite il nascondimento della sua identità "privata", l'eroina compie il suo programma narrativo trasformando il messaggio da potenzialmente personale in universale e in linea con il personaggio, nato appunto da un collettivo.

In ultima analisi, quindi, sono proprio questi artefatti, utilizzati di norma per occultare ma che qui al contrario sono indispensabili per permettere l'identificazione del personaggio, a rendere possibile la "personificazione" (Greimas e Courtès 1982, 240) dell’oggetto M¥SS KETA nella persona (collettiva) M¥SS KETA, dotandola di un programma narrativo (collettivo) e permettendole di esercitare un fare (che è, ancora una volta, collettivo).

\section{Le tematiche delle canzoni}

Visto, a questo punto, qual è stato il percorso che ha portato alla creazione della persona collettiva M¥SS KETA e definite le modalità (e i significati, attribuiti e attribuibili) degli accessori che la caratterizzano, può essere utile un rapido sguardo alle tematiche affrontate nelle sue canzoni.

Per comprendere appieno le possibili declinazioni di un prodotto culturale è però impossibile prescindere completamente dal contesto sociale che l'ha generato (Lévi-Strauss 1985 , p. 10). Si è già accennato al forte radicamento geo-

27. Prova che è facilmente identificabile con i primi lavori dell'artista.

28. Il travestimento di M¥SS KETA può essere ricondotto al genere del "nuovo e sempre uguale". Infatti, la maschera è sempre composta dalla combinazione veletta-occhiale, accessori che però l'artista (e il collettivo che ne detta le mosse) amano cambiare e personalizzare a seconda del contesto e dell'occasione. 
M¥SS KETA

come

persona collettiva

grafico di questa artista ${ }^{29}$ che, dal punto di vista dell'immaginario che l'ha generata, prende origine dalla cosiddetta "Milano da bere" ${ }^{30}$ rileggendo questo contesto con la lente dell'ironia e dell'esasperazione. Questa esasperata ironia rispetto a quelle che si potrebbero interpretare come le origini del suo stesso "mito” permette, di conseguenza, a M¥SS KETA di paragonare il passato alla contemporaneità ${ }^{31}$ e di collocarsi, come icona, al di fuori del tempo.

I testi delle canzoni citano in larga parte le tematiche più comuni nel genere hip-hop ${ }^{32}$ ma non mancano interessanti riferimenti a una nuova femminilità. Qui l'aspetto fisico è, ancora una volta, esasperato ma il centro della rappresentazione non è una sessualizzazione dei corpi bensì un'auspicata dimensione collettiva e condivisa all'interno della quale la messa in mostra del corpo diventa una scelta autonoma e auto-riferita che non si sottrae allo sguardo altrui ma che non fa dello sguardo degli altri un obiettivo al quale tendere. Anche in questo senso la dimensione collettiva di M¥SS KETA è centrale ${ }^{33}$.

Il nascondimento del viso e la possibilità di affrontare, forte della maschera che indossa, anche argomenti "scomodi" rappresenta poi il terzo punto centrale nelle tematiche

29. “M¥SS KETA è di Milano, e non potrebbe essere altrimenti” (M¥SS KETA, 2019, p. 1).

30. Questa espressione, nata nel contesto pubblicitario (con lo spot "Amaro Ramazzotti Milano da bere" ideato nel 1985 da Marco Mignani), è entrata rapidamente a far parte del linguaggio giornalistico (Amendola, 2015, p. 10). A oggi l'etichetta è utilizzata per descrivere un periodo (identificabile, convenzionalmente, tra il 1981 della fine degli anni di piombo, e il 1992 dell'inizio dello scandalo Tangentopoli), di carrierismo esasperato e comune percezione di un ampio benessere e di un diffuso edonismo.

31. Un esempio chiaro è il testo della canzone d'esordio "Milano, sushi \& coca" (2014) dove convivono riferimenti legati alla stretta attualità (come l'ascesa al mainstream delle pietanze orientali presenti anche nel titolo e assurte a status symbol o il riferimento agli hashtag) e le citazioni alla cocaina e al trionfo dell'industria della moda, tipicamente radicate nel contesto della Milano anni Ottanta. Il brano "Adoro" (2019), realizzato in collaborazione con Il Pagante, è invece più radicato al contesto attuale e le strofe rappate si alternano a messaggi vocali registrati in momenti diversi della notte.

32. Dai riferimenti (quasi innumerevoli) ai consumi di droghe di vario genere ai marchi di moda (un tema molto amato dal genere hip-hop, si pensi per esempio al brano "No Stylist" di French Montana $f t$. Drake), dalle critiche alla classe dirigente e politica fino al racconto della quotidianità in condizioni di marginalità ed emarginazione.

33. Un'importanza cruciale è quella ricoperta dal già citato videoclip "Le ragazze di Porta Venezia - The Manifesto" (2019) che, in questo senso, sarebbe interessante confrontare, purtroppo non in questa sede, con il videoclip di Cyndi Lauper "Girls just want to have fun" (1983). 
toccate dall'artista. Tanto nei brani quanto nel libro "autobiografico" l'artista non risparmia pesanti critiche, come già detto, alla classe politica dei tre decenni all'interno dei quali "si muove" (Anni Ottanta, Anni Novanta e Anni Duemila), alla chiesa cattolica e alla sua ritualità ${ }^{34}$.

\section{Conclusioni}

M¥SS KETA è un fenomeno contemporaneo ma avulso dal tempo cronologico che, grazie alla dimensione collettiva che la caratterizza fin dalla sua genesi, riesce a proporsi come emblema di periodi diversi facendosi portatrice di messaggi universali, ancorché radicati geograficamente nella loro "milanesità". Questi messaggi sono sempre passibili di interpretazioni diverse e, sfruttando il meccanismo ludico del "far finta di", instaurano con i destinatari della comunicazione un rapporto basato su una cosciente ironia.

M¥SS KETA è un personaggio prototipico che, distaccandosi dalla sua concreta dimensione biologica, utilizza la superficie anatomica del suo corpo come palcoscenico per lo svolgimento di una rappresentazione. L'anonimato assoluto è la prima caratteristica della performer ma anche ciò che le permette, da un lato, di affrontare tematiche diverse e, dall'altro lato, di favorire un processo di identificazione che la rende un'icona, un manifesto. L'insieme di queste caratteristiche permettono, di conseguenza, a M¥SS KETA di proporsi all'interlocutore come il manifesto di un sentimento universale.

Il caso di quest'artista, infine, è interessante anche perché permette una riflessione più generale su una particolare idea di persona che sembra, in qualche modo, sganciarsi dall'individualismo marcato che pare egemone nella modernità ${ }^{35}$. M¥SS KETA, da parte sua, incarna infatti una tendenza inversa e - facendosi "testimonial" di un ritorno al passato - si rifà evidentemente all'origine del termine "persona" intesa come "maschera" sia nella sua derivazione dal

34. Tra le tante citazioni possibili su questo soggetto si pensi, per esempio, alla strofa: «Anni 2000 dico, Ł̀ quello giusto / Si chiamava Wojtyla / Aveva il corpo di Cristo» (in "UNA DONNA CHE CONTA", 2018) o, in “Xananaxs” (2017), «Sono la gran contessa / Arciduchessa, sacerdotessa / Controversa, compromessa /La prima donna a dire la messa».

35. A questo proposito si veda, per esempio, il contributo di Carlo Capello ospitato in questo stesso volume. 
M¥SS KETA greco $\pi \rho o ́ \sigma \omega \pi$ ov (prōsōpon) "ciò che sta davanti alla vista", sia nel suo riferirsi in maniera letterale all'altra origine latina che, partendo dal verbo sonare, identifica l'origine del termine persona nella fusione di "per" e "sonare" ("suonare attraverso") attribuendo alla maschera lo status di megafono attraverso cui la voce risuona in maniera amplificata. Lartista senza volto tramite quella giustapposizione di accessori che diventa la sua maschera si fa dunque maschera a sua volta e non solo permette alla sua voce di essere udita ma permette al suo stesso personaggio di esistere. E questa è la concreta dimostrazione del fatto che la trasfigurazione da persona a persona collettiva è perfettamente realizzata.

Bibliografia

Amendola G.

2015 Le emozioni e la città: dalla Sindrome di Stendhal all'emotional city marketing, «Territorio», vol. 73/2, pp. 7-12.

Bonvecchio C.

2007 La Maschera e l'uomo, FrancoAngeli, Milano.

Borroni M.

2004 Rime di sfide, Arcipelago Edizioni, Milano.

Calabrese $\mathrm{O}$.

2011 L'arte del trompe-l'oeil, Jaca Book, Milano.

Conrad K., Dixon T.L., Zhang Y.

2009 Controversial Rap Themes, Gender Portrayals and Skin Tone Distortion: A Content Analysis of Rap Music Videos, «Journal of Broadcasting \& Electronic Media», vol. 53 /1, pp. 134-156.

Greimas A., Courtès J.

1979 Sémiotique. Dictionnaire raisonné de la théorie du langage, Hachette, Paris; trad. it. Semiotica. Dizionario ragionato della teoria del linguaggio, a cura di P. Fabbri P., Bruno Mondadori, Milano, 2007.

Jung C.G.

1965 Simboli della trasformazione, in Opere, 5, Bollati Boringhieri, Torino.

Kubrin C.

2005 Gangstas, Thugs, and Hustlas: Identity and the Code of the Street in Rap Music, «Social Problems», vol. 52, pp. 360378.

Lévi-Strauss C.

1975 La Voie des masques, Plon, Paris; trad. it. La via delle maschere, a cura di P. Levi, Einaudi, Torino, 1985. 
M¥SS KETA

2019 Una Donna che conta, Rizzoli, Milano.

Paolucci C.

2020 Persona. Semiotica dell'enunciazione e filosofia della soggettività, Bompiani, Milano.

Propp V.

2000 Morfologia della fiaba, Einaudi, Torino.

Volli U.

1997 Fascino, Feticismi e altre idolatrie, Feltrinelli, Milano

2000 Manuale di semiotica, Laterza, Roma-Bari.

\section{Zaggia $\mathrm{N}$.}

2019 Du féminisme en caps lock, «TraHs», n. 6 (Sexe majeur, sexe mineur?), a cura di M.G. Raout, pp. 148-165. 
"Fine" della persona

Jenny Ponzo
Ora, quel che fa problema è il semplice fatto che la persona esiste sotto il regime di una vita che si snoda dalla nascita alla morte. In cosa consiste quel che si può definire la concatenazione di una vita?

Ricoeur (1997, p. 64)

Quale conclusione più pertinente per questo volume che proporre una riflessione - o meglio una breve digressione - sulla nozione di persona secondo la categoria della "finitezza"*? Questa riflessione, condotta da una prospettiva di tipo semiotico, non ha chiaramente alcuna pretesa di esaustività, ma intende riprendere e approfondire alcuni dei numerosi e importanti stimoli emersi nei contributi raccolti in questo libro e lanciare a sua volta qualche piccolo spunto per ulteriori approfondimenti sull'idea di persona, così centrale e rilevante nella nostra cultura $\mathrm{o}$, per meglio dire, civiltà, nel senso più ampio e nobile del termine.

1. Fine come "scopo" e come "finale"

In linguistica, per persona si intende la categoria con cui le lingue naturali si riferiscono ai partecipanti all'evento comunicativo (Beccaria 1994, p. 585). In relazione alla teoria

* Questo scritto si colloca nel quadro del progetto NeMoSanctI, che ha ricevuto finanziamenti dal Consiglio europeo della ricerca (CER) nell'ambito del programma di ricerca e innovazione Orizzonte 2020 dell'Unione europea, in virtù della convenzione di sovvenzione n. 757314 (per maggiori informazioni sul progetto, vedi il sito Internet: nemosancti.eu). 
dell'enunciazione, la persona può essere definita come un'istanza che permette di esprimere la soggettività mediante il linguaggio ${ }^{1}$. Le persone si possono quindi pensare come agenti che si relazionano tra loro negli enunciati e in molti casi sono soggetti di azioni. Per la semiotica greimasiana, il soggetto è in effetti un agente la cui azione è fin da subito orientata. Il soggetto, infatti, diventa tale soltanto in relazione a un oggetto di valore da realizzare, ossia nel momento in cui "prende in carico" una certa missione per il raggiungimento di un certo obiettivo (Greimas e Courtès 1979, pp. 76-77). La persona può quindi essere considerata anche come un soggetto che agisce, e agisce in base a uno scopo: la sua azione è volta alla realizzazione di un valore o, per essere più precisi, di un sistema di valori (sulla definizione di persona in base agli atti si veda l'intervento di Francesco Galofaro in questo volume, che esplora il pensiero di Karol Woytila). Come ho dimostrato altrove, i valori che spingono i soggetti ad agire possono essere di diverso tipo, da quelli più materiali e contingenti a quelli umanitari, spirituali $\mathrm{e}$ trascendenti (Ponzo 2015). La persona in quanto soggetto è dunque fin da subito proiettata in una dimensione narrativa, in una storia, e dunque nello spazio e nel tempo: la persona è un soggetto orientato verso il raggiungimento di uno scopo e pertanto "ha una storia". Da questo punto di vista, essere considerati e considerare se stessi "persone" significa rivestire uno o più ruoli attanziali e tematici, che si collocano a un livello più profondo delle nostre caratteristiche idiolettiche, quei tratti peculiari e individuali che segnano le nostre differenze dagli altri ${ }^{2}$.

Questo fondamentale orientamento della persona verso un fine si può mettere in relazione con una tendenza rilevata da molti studiosi di letteratura e fiction in generale. Per esempio, Frank Kermode (1967) ha affermato che gli esseri umani hanno un innato bisogno di un/a fine e di conseguenza spesso vanno alla ricerca del senso della loro vita fruendo storie che hanno dei finali: da questo punto

1. Per un approfondimento sulla nozione di persona in relazione alla questione della soggettività nell'enunciazione, si veda Paolucci (2020, pp. 39-43).

2. Sulla dimensione narrativa insita nel concetto di persona, si veda il pensiero di Ricoeur, che riprende e riformula il pensiero di Emmanuel Mounier, si veda Ricoeur (1997, pp. 24, 64-71). 
di vista, sapere che la storia segue un filo logico fino alla fine è rassicurante, sicuramente più che essere immersi in un eterno e caotico presente, in cui è difficile a volte capire in che direzione e verso quali fini muoversi. La fine è dunque insita nelle strutture narrative, e in un certo senso le determina e conferisce loro un significato. Un concetto in qualche modo simile è espresso da Umberto Eco (1984) nelle Postille a Il nome della Rosa, dove afferma che il successo del genere giallo si spiega con il fatto che il lettore è tenuto avvinto dal desiderio di comprendere come gli eventi che sono terminati in un delitto e che appaiono a prima vista inspiegabili si concatenino in realtà in una sequenza logica. La ricostruzione di questa catena logica insegna qualcosa su di sé al lettore stesso, ma dato che la logica del delitto è di fatto dettata dal colpevole e dai suoi "moventi", ne deriva per Eco che ciò che i gialli dimostrano di fatto è che i colpevoli siamo noi lettori, che finiamo per interpretare tutta la storia alla luce dei "moventi", degli scopi dei soggetti "colpevoli”, con cui così finiamo fatalmente per identificarci ${ }^{3}$.

La soggettività, tuttavia, non è condizione necessaria e sufficiente a definire una persona. Lo dimostra chiaramente un modo ricorrente di rappresentare soggetti privati della possibilità di agire secondo i loro scopi e valori, come ad esempio coloro che si trovano imprigionati in carceri e campi di concentramento o in condizioni di grave malattia, o i bambini che devono subire le decisioni degli adulti. In questi casi, la soggettività stessa è messa in discussione e gli individui che patiscono queste condizioni sono spesso descritti come "persone" che hanno però subito un processo di reificazione (Ponzo 2015, pp. 95-138, 287-353) che ne compromette la agency. Data la stretta parentela tra la nozione di soggettività e l'idea di persona, non stupisce che nel discorso su tali soggetti deprivati della loro soggettività si ricorra spesso - e in termini polemici - alla nozione di persona, contrapposta all'idea di "cosa" e di "bestia" (intesa come peggiorativo o dispregiativo di "animale, non umano"). Solo per fare qualche esempio, il discorso dei cattolici sottolinea spesso che sono "persone" il feto fin dal concepimento (Lee 2012) e il carcerato, privato della sua

3. Su questo, si veda anche Ponzo (2014). 
libertà; simili metafore e similitudini si trovano anche in letteratura, un esempio per tutti è dato dalla Strage dimenticata di Andrea Camilleri (1984), saggio storico ambientato nel Risorgimento, in cui è ricorrente la similitudine del detenuto trattato come una bestia o come un utensile da lavoro (Ponzo 2015, pp. 330-335). In questi discorsi, accade che determinati esseri umani siano considerati "persone" e dunque in quanto tali inseriti in un certo sistema attanziale e in un certo ruolo tematico; le circostanze, tuttavia, sono presentate come tali da inibire la loro soggettività, impedendone la piena realizzazione. Questo impedimento, in tali tipi di discorsi, è naturalmente un elemento disforico e dalle connotazioni morali negative. Si ha in tutti questi casi un programma narrativo - una storia - in potenza, che per ragioni esterne e coercitive non riesce ad attuarsi come dovrebbe: pur non essendo dotati di "soggettività", questi individui sono ritenuti comunque "persone", ma persone la cui naturale tensione verso un fine è - momentaneamente o definitivamente - inibita.

2. Fine come "finis", confine e limite

La situazione della persona privata della sua soggettività ci porta ad esplorare un altro senso della "finitezza" intrinseca a questa nozione, vale a dire l'idea del finis latino, inteso come "limite" e come "confine". Di certo, come già aveva teorizzato Mauss (1938), l'idea di persona è il risultato di una continua negoziazione, in cui a essere messi costantemente in discussione sono proprio i confini. I dibattiti in bioetica dimostrano come tali confini non siano fissi, ma cambiano nel tempo e in diverse culture e sottoculture. Risulta difficile e controverso in particolare definire i limiti spazio-temporali della vita degli individui. Da tale difficoltà si evince che è chiara e condivisa una idea generale di persona nei suoi sèmi nucleari, mentre l'incertezza cresce man mano che ci si allontana da queste caratteristiche centrali. I confini della nozione di persona appaiono incerti in particolare secondo tre coordinate: l'inizio (si pensi alla controversa definizione dello status del feto), la fine (si pensi alle difficoltà che la nostra cultura ha nel gestire le problematiche del fine vita e, negli ultimi anni, della persistenza delle tracce online della persona dopo la morte, si veda l'intervento di Davide Sisto in questo volume), e l'ampiezza della categoria. Rientrano 
in quest'ultima problematica i dibattiti sullo status degli animali proprio in relazione alla nozione di persona ${ }^{4}$.

L'idea di confine porta inevitabilmente alla constatazione della relazionalità dell'essere umano: la frontiera implica sempre la presenza di una alterità, di uno spazio contrapposto (Leone 2007). La persona funziona in modo simile a una piccola semiosfera, con un nocciolo di credenze, di idee, una enciclopedia di segni e testi stabili nel centro e sempre più fluttuanti ed elastici verso la periferia, con bordi permeabili, che effettuano continuamente operazioni di traduzione nei confronti di segni e testi che vengono dal mondo esterno e dalle altre persone. Potremmo dunque spingerci a ipotizzare che il "valore" di una persona si può misurare in senso saussuriano, vale a dire in termini differenziali: ricordiamo che per Saussure (1916) il valore di un segno si definisce non in assoluto, ma in relazione agli altri; lo stesso vale per la persona, la quale definisce la propria specificità proprio nel rapporto con gli altri.

Da queste poche considerazioni emerge chiaramente quanto conti il limite nella definizione della persona. Come già abbiamo accennato, sono proprio i casi limite che spingono a una riflessione sulla persona: se esiste un nucleo di sèmi nucleari saldamente ancorati al segno persona, un numero piuttosto elevato di sèmi contestuali, il cui significato evolve nel corso del tempo, è oggetto di innumerevoli dibattiti. Già William James (1902), nel suo studio sulla conversione religiosa, suggeriva l'importanza dello studio dei casi più estremi, dei casi limite, per comprendere un determinato fenomeno: allo stesso modo, la definizione del concetto di persona passa per lo più attraverso un continuo processo di negoziazione dei suoi limiti, o, per dirla con Zilberberg (1993), delle sue "soglie", spostare le quali ha grandi ricadute non soltanto semiotiche, ma anche e soprattutto morali e sociali ${ }^{5}$.

Esplorare il limite pare dunque un mezzo euristico di fondamentale importanza per la definizione del concetto di

4. Su cui si veda Leone (2021), ma anche Ventura Bordenca (2018).

5. Proprio per questo motivo, il progetto di ricerca NeMoSanctI (si veda la nota 1), si concentra sui santi, come individui senza dubbio eccezionali ma anche proposti all'imitazione dei fedeli: molto spesso, i santi, con le loro parole e opere, hanno un ruolo importante appunto nello spostare le soglie del senso comune e di importanti concetti religiosi. Si veda ad esempio Ponzo (2020). 
persona. Questo vale non soltanto dal punto di vista della ricerca semiotica e della riflessione filosofica, culturale e politica, ma anche dal punto di vista dell'esperienza personale e sociale.

Tuttavia, la nostra finitezza ci impone, drammaticamente, un forte limite prospettico, che ci rende difficile una autentica conoscenza di noi stessi e dell'Altro (è quello che Ugo Volli, giustamente, definisce "problema del solipsismo" nel suo contributo); eppure, proprio l'esplorazione dell'area incerta in cui la nostra "vista" si fa sfuocata è indispensabile. In questo senso, la liminalità si pone come dimensione cognitiva e spaziale privilegiata per la scoperta e la crescita della persona. Tale liminalità può assumere molte forme, si pensi ad esempio a quella che caratterizza il rito di iniziazione descritto in etnografia e antropologia ${ }^{6}$, ma anche alle storie di conversione in cui una nuova conoscenza di se stessi e una ridefinizione del proprio sistema di valori ha luogo in situazioni estreme, staccate dal tempo e dallo spazio della vita di tutti i giorni e collocate invece in una dimensione spaziale e/o cognitiva ${ }^{7}$ eccezionale $^{8}$. Oppure, si pensi ancora alla teoria della formazione proposta in questo volume da Sara Nosari, in cui la persona è concepita proprio nell'infinita tensione in cui sfida i propri limiti per coltivare la propria perfettibilità. In molti casi, infine, la via per la comprensione della persona passa per un radicale rifiuto dei suoi limiti: come argomenta Carlo Capello, l'idea antropologica di persona si basa sul superamento del concetto di individualità. In questo senso pare particolarmente pertinente e rappresentativo il pensiero di Peirce, il quale, come spiega Emanuele Fadda, contrappone nettamente la persona(lità) appunto agli angusti e sterili confini della individualità.

Altro caso in cui l'idea di persona scavalca i limiti dell'individualità è dato da quelle persone che incarnano una forma di vita, diventando personaggi esemplari, prototipi di ruoli tematici, emblemi di un sistema di credenze o di uno

6. Si vedano ad esempio Van Gennep (1909) e Turner (1967).

7. È questa la dimensione delle esperienze mistiche. Per riflessioni sul linguaggio mistico, si veda ad esempio Galofaro (2019 a,b,c).

8. Si pensi ad esempio al topos ricorrente in fiction del viaggio o della permanenza in luoghi e situazioni "altri" come occasione di (ri)scoperta di sé e di cambiamento. 
stile di azione. Nel suo saggio, Eleonora Chiais propone un esempio concreto e contemporaneo di persona che supera la propria individualità spogliandosi del proprio io per diventare maschera o "persona collettiva". Altro esempio di trasformazione della persona individuale in "personaggio", funzionale a incarnare una certa concezione religiosa, è dato da Stefano Traini, con la sua analisi della rappresentazione paolina della figura di Gesù.

Sempre nella direzione del superamento dei limiti, questa volta materiali, vanno anche le idee di personhood virtuali, del tutto o in parte svincolate dall'esistenza di un corpo fisico. È questo tradizionalmente il caso di personaggi religiosi (si veda in merito il contributo di Marco Papasidero) che si presentano come agenti sebbene privi di un corpo materiale, ma in anni recenti si stanno rapidamente sviluppando anche idee e tipi di persone virtuali e/o artificiali del tutto nuovi grazie alla tecnologia, come dimostrano gli interventi di Davide Sisto, Gianmarco Giuliana e Alessandro Agnese. La lunga storia del concetto di persona rimane dunque aperta, anzi sta conoscendo nel nostro tempo uno straordinario sviluppo. Speriamo che, in questo periodo di rapidi cambiamenti sia tecnologici sia sociali, questo volume sia riuscito a indurre lettori e lettrici a soffermare il loro sguardo su declinazioni antiche e moderne di questa nozione chiave, e che abbia regalato loro nuove prospettive sul suo significato e valore e, perché no, su se stessi come persone.

\section{Bibliografia}

Beccaria G.L. (a cura)

1994 Dizionario di linguistica, Einaudi, Torino.

Camilleri A.

1984 La strage dimenticata, Sellerio, Palermo.

Eco U.

1984 Postille a Il nome della rosa, Bompiani, Milano. Galofaro F.

2019a Mistica e linguaggio ai primi del '900. Teresa di Lisieux e Gemma Galgani, in J. Ponzo, F. Galofaro (a cura di), Semiotica e santità. Prospettive interdisciplinari, CIRCe, Torino, pp. 86-106 (https://issuu.com/nemosancti/docs/ semiotica-e-santita-nemosancti-circe-2019).

2019b Mistica e linguaggio ai primi del '900. Padre Pio apprendista mistico", in J. Ponzo, F. Galofaro (a cura di), Semioti- 
ca e santità. Prospettive interdisciplinari, CIRCe, Torino, pp. 109-120 (https://issuu.com/nemosancti/docs/semiotica-e-santita-nemosancti-circe-2019).

2019c Mistica e linguaggio ai primi del '900. Il rapporto tra Padre Pio e Gemma Galgani", in J. Ponzo, F. Galofaro (a cura di), Semiotica e santità. Prospettive interdisciplinari, CIRCe, Torino, pp. 123-144 (https://issuu.com/nemosancti/docs/semiotica-e-santita-nemosancti-circe-2019).

Greimas A.J., Courtès, J.

1979 Sémiotique: dictionnaire raisonné de la théorie du langage, Hachette, Paris.

James W.

1902 The Varieties of Religious Experience: a Study in Human Nature, Longmans, Green \& Co., New York.

Kermode F.

1967 The sense of an ending. Studies in the theory of fiction, Oxford University Press, New York.

Lee P.

2012 Abortion and Unborn Human Life, Second Edition, The Catholic University of America Press, Washington.

Leone $\mathrm{M}$.

2007 Appunti per una semiotica della frontiera, «Solima», http:// solima.media.unisi.it/documenti/Leone_\%20Appunti_semiotica_frontiera.pdf.

2021 On Muzzles and Faces. The Semiotic Limits of Visage and Personhood, "International Journal for the Semiotics of Law», https://link.springer.com/article/10.1007/ s 1 1196-020-09812-8? fbclid=IwAR3Bi0_Z9KEODyKejnAKsjD69-4h51SUoiNC3AW04UGTeF8owjf40y66c2A

Mauss M.

1938 Une Catégorie de l'esprit humain: la notion de personne celle de 'moi', "The Journal of the Royal Anthropological Institute of Great Britain and Ireland», 68, pp. 263-281.

Paolucci C.

2020 Persona. Soggettività nel linguaggio e semiotica dell'enunciazione, Bompiani, Milano.

Ponzo J.

2014 'Les coupables, c'est nous': symboles et indices pour une étude du roman historique, «A contrario», 20, pp. 129-142.

2015 La narrativa di argomento risorgimentale (1948-2011), Aracne, Roma.

2020 The case of the 'offering of life' in the causes for canonization of Catholic saints: the threshold of self-sacrifice, "Interna- 

springer.com/article/10.1007/s1 1196-020-09713-w.

\section{Ricoeur P.}

1997 La persona, Morcelliana, Brescia.

Saussure F. de

1916 Cours de linguistique générale, Payot, Paris.

Turner V.

1967 The forest of symbols. Aspects of Ndembu ritual, Cornell University Press, Ithaca - New York.

Van Gennep A.

1909 Les rites de passage, Nourry, Paris.

Ventura Bordenca I.

2018 Martyrdom in Contemporary Animalist Discourse, «Lexia», 31-32, pp. 315-336.

\section{Zilberberg C.}

1993 Seuils, limites, valeurs, in On the Borderlines of Semiotics: proceedings from the ISI conferences 1991 and 1992 in Imatra («Acta Semiotica Fennica II»), Imatra ISI, Helsinki, pp. 379-395. . 
aA

finito di stampare stampato nel mese di settembre 2021 per i tipi di Accademia University Press in Torino 